University of Vermont

UVM ScholarWorks

2019

\title{
Acoustic Emission Sensing for Crack Monitoring in Prefabricated and Prestressed Reinforced Concrete Bridge Girders
}

Robert Lee Worley II

University of Vermont

Follow this and additional works at: https://scholarworks.uvm.edu/graddis

Part of the Civil Engineering Commons

\section{Recommended Citation}

Worley, Robert Lee II, "Acoustic Emission Sensing for Crack Monitoring in Prefabricated and Prestressed Reinforced Concrete Bridge Girders" (2019). Graduate College Dissertations and Theses. 1020.

https://scholarworks.uvm.edu/graddis/1020

This Thesis is brought to you for free and open access by the Dissertations and Theses at UVM ScholarWorks. It has been accepted for inclusion in Graduate College Dissertations and Theses by an authorized administrator of UVM ScholarWorks. For more information, please contact scholarworks@uvm.edu. 


\title{
ACOUSTIC EMISSION SENSING FOR CRACK MONITORING IN PREFABRICATED AND PRESTRESSED REINFORCED CONCRETE BRIDGE GIRDERS
}

\author{
A Thesis Presented \\ by \\ Robert Lee Worley II \\ to \\ The Faculty of the Graduate College \\ of \\ The University of Vermont \\ In Partial Fulfillment of the Requirements \\ for the Degree of Master of Science \\ Specializing in Civil and Environmental Engineering
}

January, 2019

Defense Date: November 2, 2018

Thesis Examination Committee:

Mandar M. Dewoolkar, Ph.D., Advisor

Dryver R. Huston, Ph.D., Co-advisor

Tian Xia, Ph.D., Chairperson Ting Tan, Ph.D.

Cynthia J. Forehand, Ph.D., Dean of the Graduate College 


\begin{abstract}
Prefabricated and pre-stressed reinforced concrete beams and girders are integral components of many highway structures, including those built by rapid construction techniques. Concerns exist regarding the development of cracks during curing, form removal, detensioning, transport, installation, and operation. Non-destructive, Acoustic Emission (AE) sensing techniques have the potential for detecting and locating cracking in prefabricated, pre-stressed concrete girders used as Prefabricated Bridge Elements and Systems (PBES) used in rapid construction practices as part of a Quality Assurance/Quality Control (QA/QC) program. AE sensing records transient elastic waves produced by the release of stored elastic energy resulting in plastic deformations (i.e., crack nucleation and growth) with an array of point sensors. The AE instrument system is relatively portable which can allow for it to be an option for both off-site fabrication QA/QC as well as onsite field $\mathrm{QA} / \mathrm{QC}$. This thesis presents a multi-stage research initiative on acoustic emission monitoring of prefabricated and pre-stressed reinforced concrete beams used in highway bridge construction during detensioning, craned removal from formwork and transport to bridge sites, along with supporting laboratory tests and numerical analysis.

The specific objectives of this research were to: 1. Identify suitable instruments to monitor pre-stressed and/or post-tensioned concrete girders for cracking activity; 2 . Design and develop a reusable instrumentation package; 3 . Measure performance and condition of concrete girders during fabrication and transport; and 4. Identify test protocols and possible accept/fix/reject criteria for structural elements based on information from monitoring system. Presented are results from laboratory, full-scale girder fabrication, and transport monitoring, along with overall conclusions and recommendations for future research.
\end{abstract}




\section{CITATIONS}

Material from this thesis has been accepted for publication in ASCE Journal of Bridge Engineering Special Edition on October 4, 2018 in the following form:

Worley II, R. L., Dewoolkar, M. M., Xia T., Farrell R., Orfeo D., Burns D., Huston D. R.. (2019). Acoustic emission sensing for crack monitoring in prefabricated and pre-stressed reinforced concrete bridge girders. ASCE Journal of Bridge Engineering, Special Edition. 


\section{ACKNOWLEDGEMENTS}

This work was funded by the Vermont Agency of Transportation (VTrans). A special thanks to VTrans personnel who assisted in various stages of conception, planning and management, including Nick van den Berg, Jonathan Razinger, Dr. Emily Parkany, Rob Young, Dr. Ian Anderson, Douglas Bonneau and Bill Ahern. Also, a special thanks to J. P. Carrara and Sons, Inc. of Middlebury, VT who granted access to their concrete facilities and transport processes and provided invaluable insights into the behavior of prestressed concrete.

I would like to acknowledge and express my gratitude to the many faculty, coworkers, friends, and family who have supported me in my graduate research endeavors. I would like to thank my advisors Dr. Mandar Dewoolkar and Dr. Dryver Huston who have both invested a great deal of time and energy, not only in my research and academics, but also in my professional development. As a 2013 graduate of the Civil and Environmental Engineering Program at the University of Vermont, it was Dr. Dewoolkar's dedication and the dedication of other influential faculty; Dr. Donna Rizzo, Dr. Ehsan Ghazanfari, Dr. John lens, and Joan Rosebush to their students both pre- and post-graduation that convinced me to return to the University of Vermont as a graduate student.

Additionally, I would like to thank Dr. Tian Xia and Dr. Ting Tan for their willingness to serve on my thesis committee and provide constructive feedback on how to improve this research. I would also like to thank all my co-workers, managers, and owner of EarthRes Group, Inc. for their initial and continued support of my decision to return to 
Vermont to pursue graduate studies and advance my technical knowledge within the Civil Engineering discipline.

I have made many friends through this journey and would like to thank them for their willingness to lend a hand whenever needed. In no particular order, I would like to thank Bijay KC, Maziar Foroutan, Soham Banerjee, Bobby Farrell, Mauricio Pereira, Dan Orfeo, and Dr. Dylan Burns.

I would like to thank my family and my parents in particular who have always supported me in everything I do, whose support I can always count on, and who maintain to be the examples of the character and integrity that I strive to match. Finally, I would like to thank my wife, Lindsay, for her constant support, her helpful advice, and her unwavering confidence in me. As a fellow undergraduate and now graduate of the Civil and Environmental Engineering Program at the University of Vermont, she never ceases to amaze me with her academic talents and her selflessness in helping others reach their goals, myself included. 


\section{TABLE OF CONTENT}

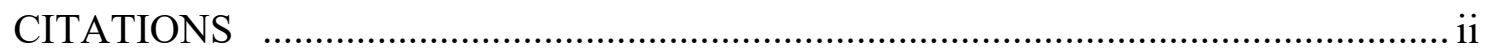

ACKNOWLEDGEMENTS ........................................................................... ii

CHAPTER 1 PROBLEM STATEMET AND RESEARCH FOCUS ......................... 1

1.1 Introduction and Motivation............................................................... 1

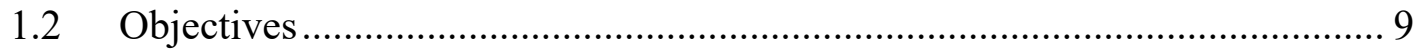

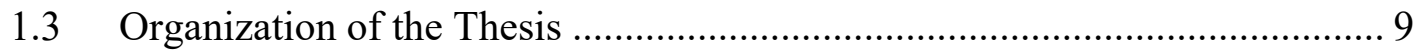

CHAPTER 2 BACKGROUND LITERATURE ..................................................... 12

2.1 Pre-Tensioned and Post-Tensioned Reinforced Concrete........................... 12

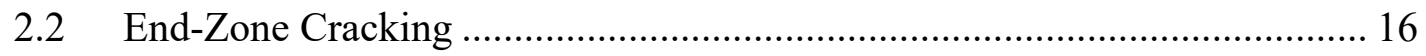

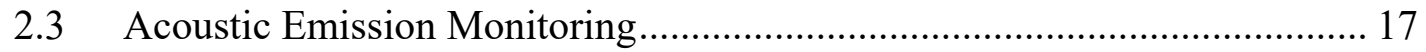

2.3.1 Overview and History .................................................................... 17

2.3.2 Acoustic Emission Sensors ............................................................. 19

2.3.3 Acoustic Emission Wave Modes and Propagation .............................. 23

2.3.4 Acoustic Emission Source Locating ................................................ 24

2.3.5 Acoustic Emission Source Differentiation............................................ 25

2.3.6 Acoustic Emission Damage Assessment ............................................ 28

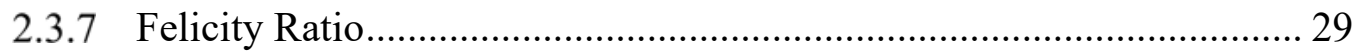

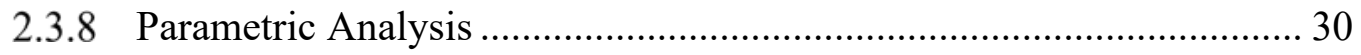

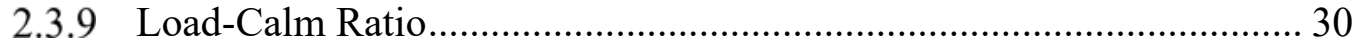

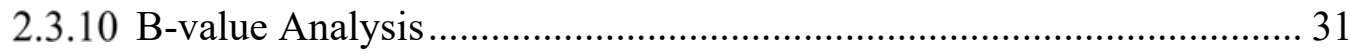

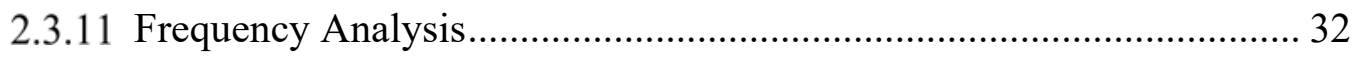




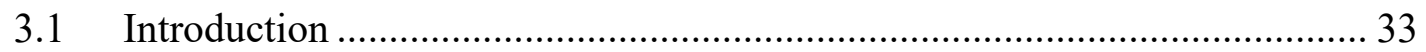

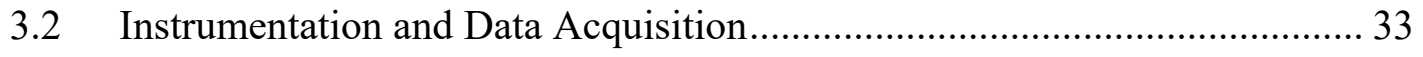

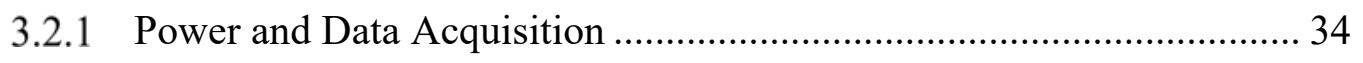

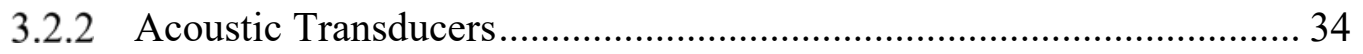

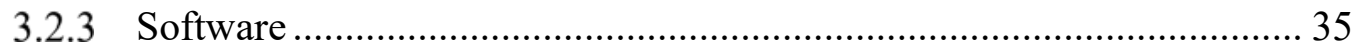

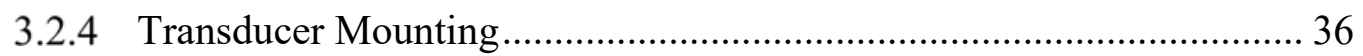

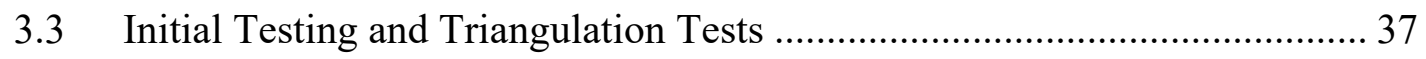

3.3.1 2-D Pencil Lead Break Testing........................................................ 39

3.3.2 3-D Pencil Lead Break Testing ...................................................... 39

3.4 AE Instrumentation Input Parameters and Data Collection ........................ 40

CHAPTER 4 ACOUSTIC EMISSION DATA COLLECTION AND RESULTS:

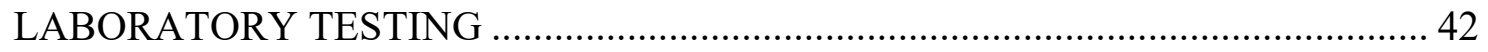

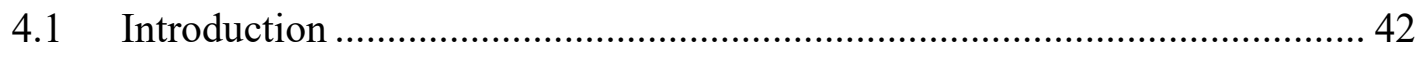

4.2 Laboratory Specimen Preparation and Load Testing Procedures ................ 42

4.2.1 Pull-out Test Data Collection............................................................ 43

4.2.2 Three-Point Bending Data Collection............................................... 44

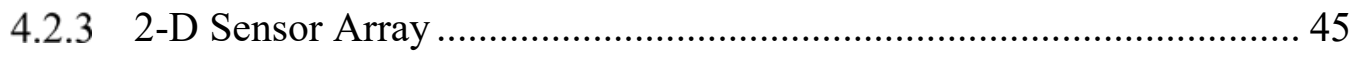

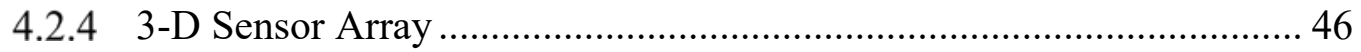

4.3 Conclusions from the Results of Laboratory Testing .............................. 47

CHAPTER 5 ACOUSTIC EMISSION DATA COLLECTION AND RESULTS:

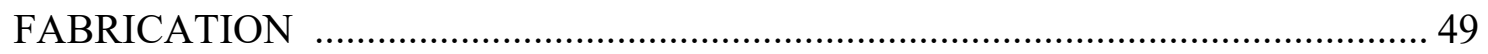

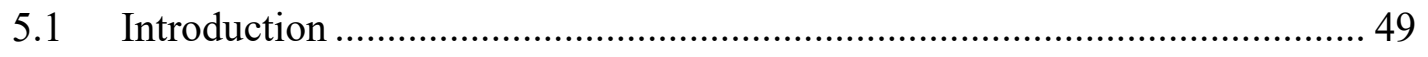

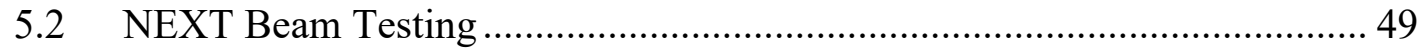




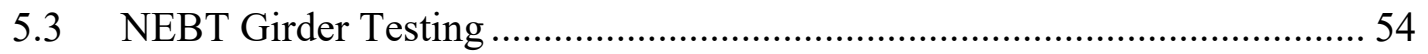

5.3.1 NEBT 3-D Sensor Array Data Collection …………………………..... 54

5.3.2 NEBT 2-D Sensor Array Data Collection ................................................. 63

5.4 Conclusions from the Testing Results of Detensioning and Lifting ............. 72

CHAPTER 6 ACOUSTIC EMISSION DATA COLLECTION AND RESULTS:

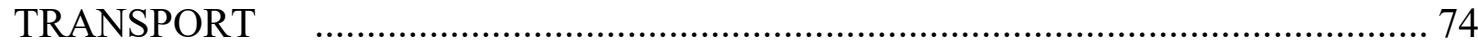

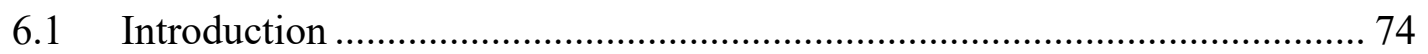

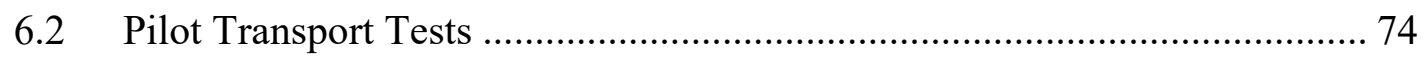

6.2.1 Instrumentation Configuration.............................................................. 75

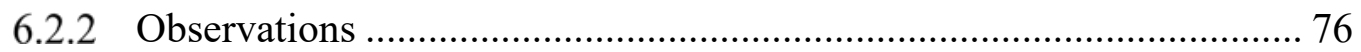

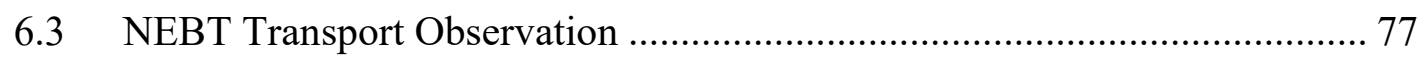

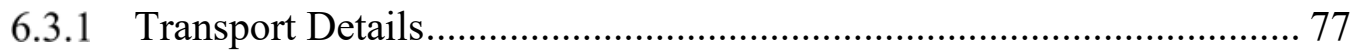

6.3.2 Transport Process Observations............................................................ 78

6.3.3 System Modifications ………………………………......................... 80

6.4 Straight NEBT End Zone Data Collection (Transport Test 1) ....................... 81

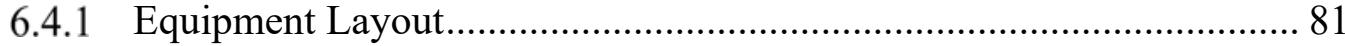

6.4.2 Representative Data Collection........................................................... 82

6.4.3 Data Collection Process Observations ..................................................... 88

6.5 Straight NEBT Mid-Span Data Collection (Transport Test 2)..................... 90

6.5.1 Equipment Layout............................................................................... 90

6.5.2 Representative Data Collection.......................................................... 90

6.5.3 Data Collection Process Observations ..................................................... 95

6.6 Hammerhead NEBT Mid-Span Data Collection (Transport Test 3)............. 99

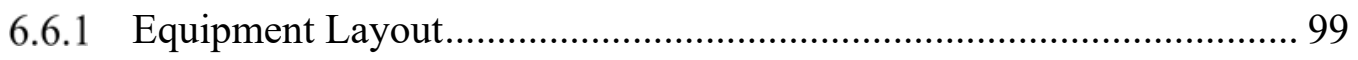

6.6.2 Representative Data Collection............................................................ 99

6.6.3 Data Collection Process Observations ……………………………..... 105 
6.7 Conclusions from the Results of Transport Testing 108

CHAPTER 7 PROPOSED METHODOLOGY FOR QUALITY ASSURANCE / QUALITY CONTROL ACCEPT AND REJECT CRITERIA ............................... 110

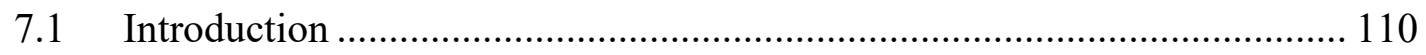

7.2 Adapted Parametric Feature Analysis ................................................ 111

7.2.1 B-value and Frequency Analyses................................................... 111

7.3 Potential AE Damage Assessment Methods .......................................... 112

7.4 Proposed Multi-Step Procedure ........................................................ 113

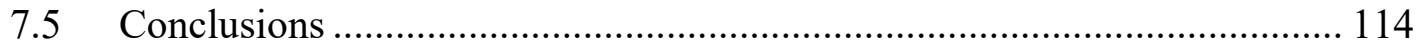

CHAPTER 8 CONCLUSIONS AND FUTURE RECOMMENDATIONS .............. 116

$8.1 \quad$ Work Performed ............................................................................. 116

8.2 Overall Conclusions .................................................................... 118

8.3 Intellectual Merit/Contributions to the Research .................................... 120

8.4 Broader Impacts of the Research................................................................. 120

8.5 Recommendations for Future Work ..................................................... 121

CHAPTER 9 COMPREHENSIVE BIBILOGRAPHY …...................................... 124

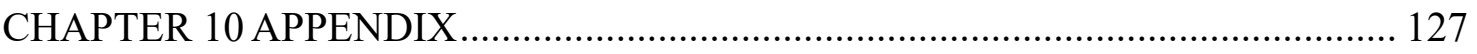

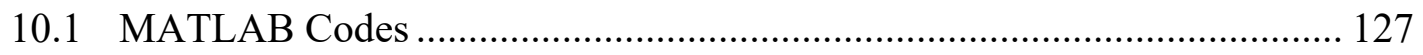

10.1.1 Example Code for Glyph Plots of Parametric Feature Data ............... 127

10.1.2 Example Code for Extracting AE Event Location Data from Raw

Data Files 127

viii 
10.1.3 Example Code for Making a Video of AE Event Capture.................... 132

10.1.4 Example Code for Plotting AE Event Locations and Source

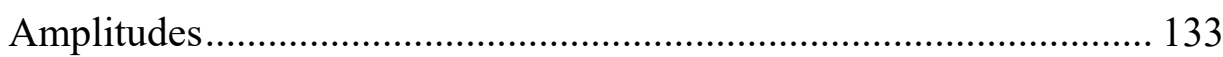




\section{LIST OF TABLES}

$\underline{\text { Table }}$

$\underline{\text { Page }}$

Table 2.1. Frequency analysis flow chart (source: Arches, 2009) ............................... 32

Table 3.1. List of Mistras AEwinTM Sensor Highway III System and Parts ................. 33

Table 3.2. Mistras AEwinTM Sensor Highway III Input Parameters ............................ 41

Table 5.1. Quantification of AE events versus number of observed cracks during

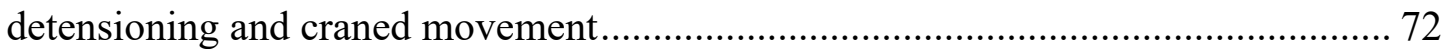

Table 6.1. Quantification of AE Events Versus Girder Type and Sensor Array Location During NEBT Detensioning and Craned Movement 109 


\section{LIST OF FIGURES}

$\underline{\text { Figure }}$

$\underline{\text { Page }}$

Figure 1.1. VT 100 Gold Creek Bridge near Moscow and Stowe with girder and deck cracks (a) bridge side view, (b) deck with multiple cracks, (c) girder with cracks in the flanges near to the end, and (d) some of the cracks repaired. 3

Figure 1.2. Acoustic emission sensor attached to a concrete girder (source: Physical

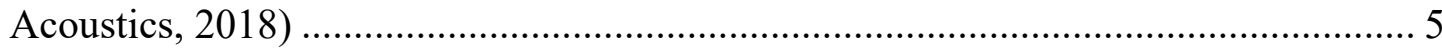

Figure 1.3. Recorded end zone cracking of NEBT girder ......................................... 8

Figure 2.1. Structural material and pre-stressed concrete types flow chart (source:

Steel Auto Industries, 2018) 13

Figure 2.2. Pre-tensioned and post-tensioned concrete (a) tensioning of the prestressing strands against the end abutments, (b) casting of the reinforced concrete beam, (c) detensioning of the pre-tensioning strands, and (d) post-tensioning of the reinforced concrete beam

Figure 2.3. Post-tensioning strands (typical) (a) unbonded post-tensioning strand and

(b) bonded post-tensioning strand (source: Vejvoda, 2018) ................................... 15

Figure 2.4. Horizontal end-zone web cracking of a NEBT girder ................................ 17

Figure 2.5. Mono-crystals vs. poly-crystals (source: Aysal, 2018) .............................. 20

Figure 2.6. Polarization of ceramic material to generate piezoelectric effect (source:

Aysal, 2018) 20

Figure 2.7. Piezoelectric effect under different circumstances (a) no stress or charge,

(b) compression, (c) tension, (d) applied voltage opposite polarity, (e) applied voltage same polarity, and (f) applied AC signal (source: Aysal, 2018).... 21

Figure 2.8. Typical piezoelectric sensor schematic (source: Vallen, 2009) ................... 22

Figure 2.9. Broadband (top) vs. resonant (bottom) sensitivity comparison (source:

Grosse and Ohtsu, 2008). 23

Figure 2.10. AE signal triangulation schematic (a) crack nucleation and AE sensors,

(b) acoustical emission of transient elastic wave, and (c) AE source location by triangulation (source: Huston, 2010) 
Figure 2.11. Parametric analysis of typical measured waveform features 26

Figure 2.12. Burst emission (top) vs. continuous emission (bottom) (source: Grosse and Ohtsu, 2008)

Figure 2.13. AE event amplitude and duration filtering for crack correlation (a)

laboratory beam and AE sensor array, (b) unfiltered AE event location, and (c)

filtered AE event locations correlated to observed cracking (source: ElBatanouney

et al., 2014) 28

Figure 2.14 Felicity effect and ratio illustration (typical) (source: Arches, 2009) .......... 30

Figure 2.15 Load-calm ratio illustration (typical) (source: Arches, 2009) ...................... 31

Figure 3.1. Sensor Highway III instrument box (a) lid closed and (b) lid open .............. 34

Figure 3.2. Acoustic emission low power, $60 \mathrm{kHz}$ PK6I sensor with integral preamplifier (source: Physical Acoustics, 2018) 35

Figure 3.3. AE sensor housing mount (a) hollow square stock AE sensor mount (b) Ushaped bracket AE sensor mount.

Figure 3.4. Fabrication of pencil lead break beam (a) reinforcing bar pattern, (b) freshly vibrated wet concrete in mold, and (c) Darwin Chamber(C) used for curing... 38

Figure 3.5. Tap testing 2-D sensor array (a) top view ( $\mathrm{x}-\mathrm{y}$ plane) (b) side view (x-z plane) 39

Figure 3.6. Tap testing 3-D sensor array (a) top view ( $\mathrm{x}-\mathrm{y}$ plane) (b) side view ( $\mathrm{x}-\mathrm{z}$ plane) 40

Figure 4.1. Laboratory pull-out test of reinforced concrete beam with 3-D sensor array. (a) Photograph of 3-D sensor array prior to testing, (b) Schematic of 3-D sensor array including AE event locations, sensors, and observed cracks 44

Figure 4.2. Laboratory three-point bending test of reinforced concrete beam with 2-D sensor array. (a) Photograph of 2-D sensor array at the completion of testing, (b) Schematic of 2-D sensor array including AE event locations, sensors, and observed cracks 45

Figure 4.3. AE event duration vs. amplitude categorized by proximity to observed cracks 46 
Figure 4.4. Laboratory three-point bending 3-D sensor array. (a) Photograph of 3-D sensor array at the completion of testing, (b) Schematic of 3-D sensor array including AE event locations, sensors, and observed cracks

Figure 5.1. NEXT beam field testing of detensioning with 38 recorded events (a) field testing equipment setup, (b) torch cutting of pre-tensioning strands, (c) plan view of AE events on NEXT beam, (d) section view of NEXT beam, and (e) profile view of NEXT beam .

Figure 5.2. NEXT beam field testing of craned form removal with 20 recorded events

(a) crane lifting, (b) plan view of AE events on NEXT beam, (c) section view of NEXT beam, and (d) profile view of NEXT beam. 53

Figure 5.3. NEBT girder 3-D sensor array field testing of detensioning with 466 recorded events (a) AE equipment setup, (b) gantry crane connection to NEBT, (c) section view of NEBT beam, and (d) profile view of NEBT beam 56

Figure 5.4. Amplitude distribution for NEBT beam 3D sensor array field testing of detensioning with 466 recorded events 57

Figure 5.5. NEBT beam 3D sensor array field testing of detensioning with 466 recorded events over time with delineation of recorded $\mathrm{AE}$ events to each torch cutting of a pre-tensioning strand 58

Figure 5.6. B-value regressions of each pre-tensioning strand for detensioning of a NEBT using a 3D sensor array

Figure 5.7. B-value of each pre-tensioning strand for detensioning of a NEBT using a $3 \mathrm{D}$ sensor array

Figure 5.8. Hammerhead NEBT girder 2D sensor array field testing of detensioning with 960 recorded events (a) AE equipment/sensor setup, (b) observed horizontal web crack, (c) section view of NEBT girder, and (d) profile view of NEBT girder .. 65

Figure 5.9. Amplitude distribution for NEBT girder 2D sensor array field testing of detensioning with 960 recorded events 66

Figure 5.10. NEBT girder 2D sensor array field testing of detensioning with 960 recorded events over time with delineation of recorded $\mathrm{AE}$ events to each torch cutting of a pre-tensioning strand 66 
Figure 5.11. B-value regressions of each pre-tensioning strand for detensioning of a NEBT using a 2D sensor array 70

Figure 5.12. B-value of each pre-tensioning strand for detensioning of a NEBT using

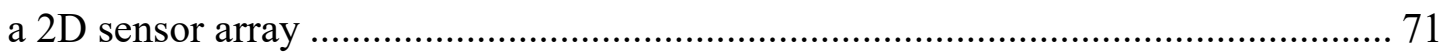

Figure 6.1. Pilot transport test AE equipment set-up................................................. 75

Figure 6.2. AE sensor connection to concrete block with U-shape brackets.................. 76

Figure 6.3. Pilot AE transport configuration showing concrete, electromechanical vibrator and transducer array, in bed of pickup truck ............................................ 76

Figure 6.4. Travel route from J.P. Carrara \& Sons Inc.in Middlebury, VT to the IM 091-1(66) I-91 bridge construction site in Rockingham, VT (source: Google Maps) 78

Figure 6.5. Transport Test 1 - AE measurements of the end zone vertical web of a straight NEBT during transport with 673 recorded AE events. (a) photograph of 2-D sensor array, (b) photograph of equipment set-up, (c) cross-section of NEBT tested, (d) profile view of NEBT including AE event locations, sensors, and observed cracks.

Figure 6.6. Transport Test 1 - AE measurements plotted by amplitude of the end zone vertical web of a straight NEBT during transport with 673 recorded AE events. 84

Figure 6.7. Amplitude distribution for a 2D sensor array transport test of the end zone of a straight section NEBT girder with 673 recorded events. 85

Figure 6.8. AE event density during transport testing of end zone region of straight NEBT, with a total event count of 623 86

Figure 6.9. Transport test TT1: b-value analyses in 30-minute time interval plots ......... 87

Figure 6.10. Transport test TT1: b-values in 30-minute time intervals 88

Figure 6.11. FEM quarter straight NEBT stress distribution with $1 \mathrm{~g}$ down, left side corresponds to mid-span and right side corresponds to the end zone.

Figure 6.12. FEM quarter straight NEBT stress distribution with $1 \mathrm{~g}$ up, left side corresponds to mid-span and right side corresponds to the end zone.

Figure 6.13. Transport Test 2 - AE measurements of the mid-span vertical web of a straight NEBT during transport with 2,628 recorded AE events. (a) photograph of 
2-D sensor array, (b) photograph of equipment set-up, (c) cross-section of NEBT tested, (d) profile view of NEBT including AE event locations, sensors, and observed cracks

Figure 6.14. Transport Test 2 - AE measurements plotted by amplitude of the midspan vertical web of a straight NEBT during transport with 2,628 recorded AE events.

Figure 6.15. Amplitude distribution for a 2D sensor array transport test of the middle zone of a straight section NEBT girder with 2,628 recorded events . 93

Figure 6.16. AE event density during transport testing of middle zone region of straight NEBT, with a total event count of 2,628 93

Figure 6.16. Transport test TT2: b-value analyses in 30-minute time interval plots........ 94

Figure 6.18. Transport test TT2: b-values in 30-minute time intervals. 95

Figure 6.19. FEM quarter straight NEBT stress distribution with 1g down, left side corresponds to mid-span and right side corresponds to the end zone 96

Figure 6.20. FEM quarter straight NEBT stress distribution with $1 \mathrm{~g}$ up, left side corresponds to mid-span and right side corresponds to the end zone

Figure 6.21. Straight span NEBT shear and bending moment diagrams during transport 98

Figure 6.22. Straight span NEBT shear and bending moment diagrams during inservice loading 98

Figure 6.23. Transport Test 3 - AE measurements of the mid-span vertical web of a hammerhead NEBT during transport with 91,723 recorded AE events. (a) photograph of girder on truck with 2-D sensor array and equipment set-up, (b) mid-span cross-section of tested NEBT, (c) profile view of NEBT including AE event locations and sensors. Note that the post-tensioning strand ducts are at a higher location in the mid-span cross sections of (b) and (c) than at the end zone as shown emerging from the end of the section face in (a). 100

Figure 6.24. Transport Test 3 - AE measurements plotted by amplitude of the end zone vertical web of a straight NEBT during transport with 91,723 recorded AE events. 
Figure 6.25. Amplitude distribution for a 2D sensor array transport test of the middle zone of a hammerhead section NEBT girder with 91,723 recorded events. 102

Figure 6.26. AE event density during transport testing of middle zone region of hammerhead NEBT, with a total event count of 91,723 ............................................ 103

Figure 6.27. Transport test TT3: b-value analyses in 30-minute time interval plots...... 104

Figure 6.28. Transport test TT3: b-values in 30-minute time intervals ......................... 104

Figure 6.29. FEM quarter hammerhead NEBT stress distribution with $1 \mathrm{~g}$ down, left side corresponds to mid-span and right side corresponds to the end zone 106

Figure 6.30. FEM quarter hammerhead NEBT stress distribution with $1 \mathrm{~g}$ up, left side corresponds to mid-span and right side corresponds to the end zone. 106

Figure 6.31. Hammerhead span NEBT shear and bending moment diagrams during transport

Figure 6.32. Hammerhead span NEBT shear and bending moment diagrams during in-service loading 107 


\section{CHAPTER 1 PROBLEM STATEMET AND RESEARCH FOCUS}

\subsection{Introduction and Motivation}

The overarching goal of this research was to develop Quality Assurance/Quality Control $(\mathrm{QA} / \mathrm{QC})$ criteria for potential cracking in prefabricated pre-stressed concrete girders used as Prefabricated Bridge Elements and Systems (PBES). The use of PBES has the potential to alter the bridge construction maintenance paradigm by minimizing construction delays while reducing costs and improving performance. However, quality control issues associated with implementing the new methods have the potential to detract and even prevent widespread use. This is exemplified by the recent construction of a short span bridge in Vermont using prefabricated post-tensioned concrete girders and a rapidcuring integral deck with significant cracking in the girders and deck. Preventing and mitigating such problems early on may be possible with the use of a properly designed reusable condition monitoring instrumentation system, when combined with effective QA/QC practices. Solving these problems will have significant implications for the construction of highway structures.

PBES techniques make extensive use of manufacturing bridge components off site and shipping as needed to the construction site, including prefabricated pre-stressed concrete girders. Advantages of these girders include strength and the ability to be lifted into place with a crane, thereby avoiding the complexity of lateral slide in place maneuvers. The girders can come with wide top flanges and top side studs to enable casting in place of an integral concrete deck immediately following girder placement. Such construction can quickly produce a strong integrated pre-stressed and reinforced deck-girder configuration. 
During this process many problems could occur that are potentially damaging to the concrete/beam at different stages of manufacturing, transport, and construction process, including:

1) Rapid load transfers from pre-stress tendon cuts,

2) Cutting the tendons before the concrete fully sets,

3) Improper lifting of the beam for transport, shipping the component,

4) Placing it in its final position, and

5) Post installation cracking often due to misalignment poor materials or miscalculated dimensions.

A significant potential drawback to using prefabricated and pre-stressed concrete girders is cracking. A recent report by Head et al. (2015) for the Maryland State Highway Administration found that many prefabricated and pre-stressed concrete bridge girders suffered from cracking, with most of the cracks appearing as diagonal cracks at the ends. These cracks were deemed unlikely to be of structural concern but were of sufficient concern to warrant further observation. Controlling camber during fabrication and delivery were also important concerns. Camber is an important component to ensuring timely placement of the girders on site. In terms of QA/QC a major issue was lack of automated inspection processes to aid in streamlining paperwork and data management.

An example of notable concrete cracking in a PBES bridge has been recently observed in Vermont. The bridge crossing Gold Creek on VT 100 near Moscow and Stowe has extensive cracking in the girders and cast-in-place integral deck. The source of these cracks has not been firmly established. It is also not certain if the cracks are stable or are 
still growing. While these cracks may not pose a serious structural threat to the bridge, they do present aesthetic, serviceability and long-term maintenance concerns.

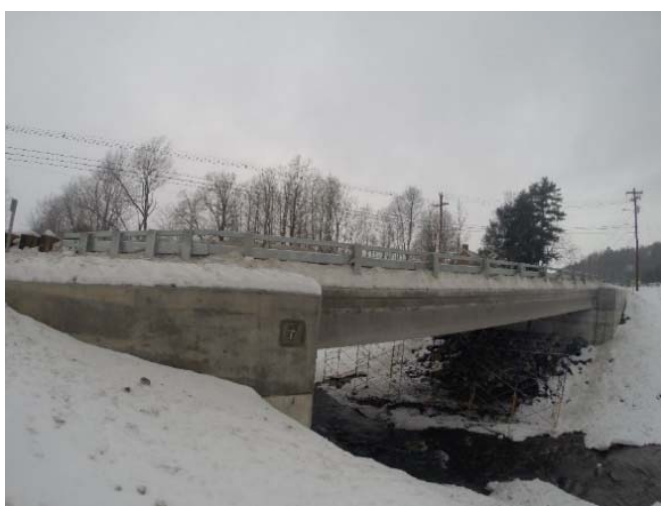

(a)

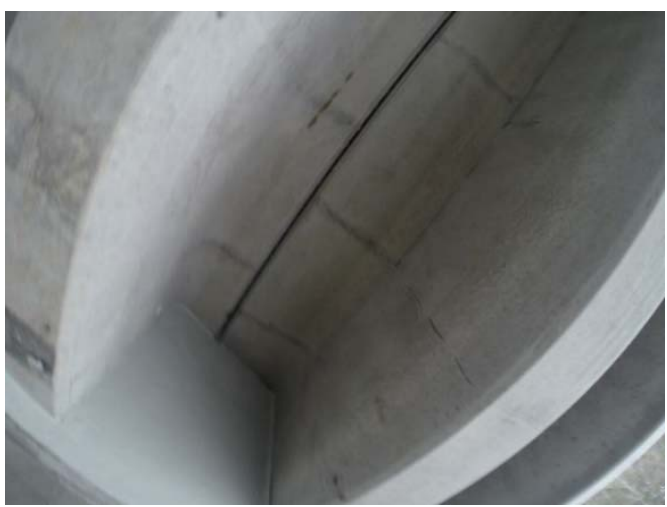

(c)

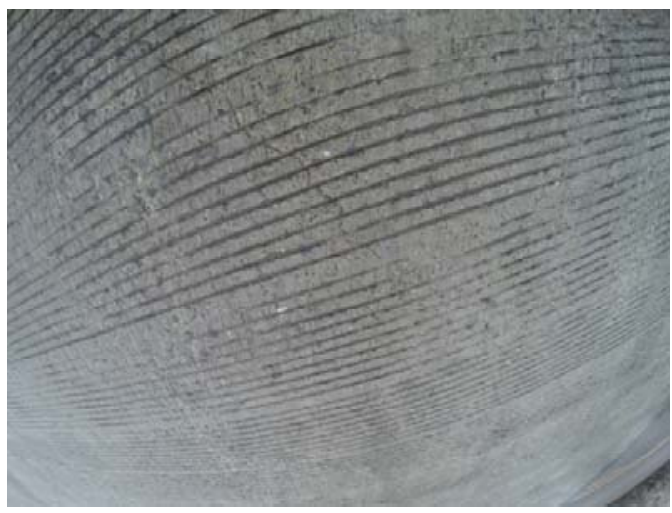

(b)

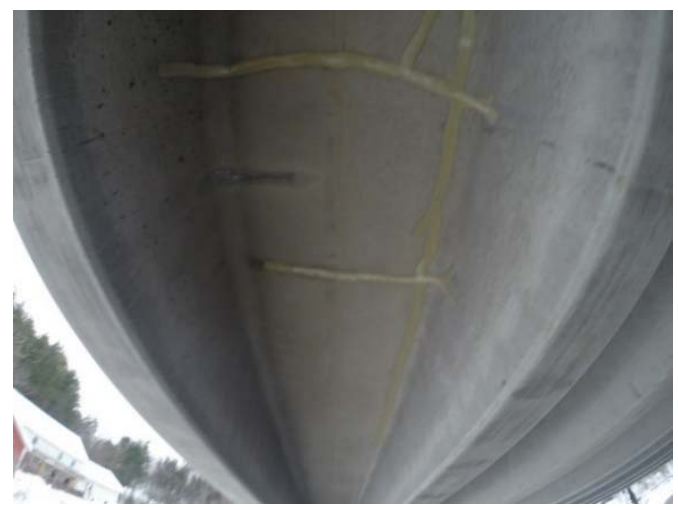

(d)

Figure 1.1. VT 100 Gold Creek Bridge near Moscow and Stowe with girder and deck cracks (a) bridge side view, (b) deck with multiple cracks, (c) girder with cracks in the flanges near to the end, and (d) some of the cracks repaired

The VT 100 Bridge along with the reports of cracking in similar girders in Maryland provides an impetus for monitoring cracks in these girders as part of a QA/QC system to minimize and mitigate this cracking problem. An ideal monitoring system would have the following traits: 1 . identify the occurrence, location and severity of cracking including non-visible subsurface cracks, 2 . provide test results in a sufficiently timely and understandable format to enable accept/reject QA/QC decision, 3. be applicable to multiple types of beams, girders and bridges, 4. do not damage the elements under test, 5. do not 
disrupt the construction process, 6 . be of sufficiently mature technology with highlyreliable and easy-to-use with turn-key instruments, 7. operate over a fairly short test cycle, and 8 . be affordable.

Structural sensing and health monitoring technologies are widespread and encompass many types of sensors (Huston, 2011). Acoustic emission (AE) monitoring appears to be the best technique for this application. AEs are short-duration high-frequency elastic waves in solids caused by incipient micro-fractures and other localized events detecting the strength, shape and timing of elastic waves emanating from cracks as they form. Analysis of the signals can determine the location and type of cracking, as well as the overall level of cracking, i.e. whether the cracks are stable or are growing. When applied to concrete girders, the simplest signal processing measures the rate of $\mathrm{AE}$ events. If the production rate is relatively low and steady or dropping, the amount of new crack generation is small. If the $\mathrm{AE}$ rate is high or growing, then the cracks are growing. Concrete AE testing has encompassed: 1. Maturity level of concrete - as concrete cures it produces micro cracking which is detectable by AE monitoring. Once the curing slows, the beam would be stable enough to move; 2 . Impact to concrete members as it is moved to its final location - $\mathrm{AE}$ can detect and locate impacts to the members; Continuous cracking; 3. Reaction of beams and components to loading; 4. Wire break in pre-stressing; and 5. Estimating load values using b-values, load-calm ratio (Landis and Baillon, 2012; Chen and He, 2001).

Advantages of AE monitoring are: 1. The sensors easily attach to the surface of the structure, are removable and reusable (Figure 1.2); 2. AE can detect, locate and assess subsurface nonvisible cracks; 3 . The technology is relatively mature with applications 
across a variety of structure types, including concrete; 4. Software and data analysis procedures are available for a variety of conditions, including those specific to concrete cracking; 5. AE-based codes, standards, test and continuous monitoring procedures (ASME, RILEM, ASTM), have been in place since the 1980s (Huang and Nissen, 1997); and 6. Certain industries, in particular pressure vessel manufacturing, use accept/reject criteria based on the level of AE signal production. The pre-stressed character of pressure vessels and pre-stressed girders are similar enough to lend credence to the possibility of developing similar accept/reject criteria for girders.

Disadvantages of AE testing are: 1. Data must be collected continuously, otherwise important $\mathrm{AE}$ events may go undetected, including those occurring prior to instrument installation; 2. The standard setup requires cables connecting $\mathrm{AE}$ sensors to the $\mathrm{AE}$ monitoring instrument. Running these cables along girders without damage requires skill and some expense. An alternative is to use wireless data transmission and incurring the associated increased costs and complexity; and 3. The monitoring instruments consume modest amounts of power $(\sim 20 \mathrm{~W})$.

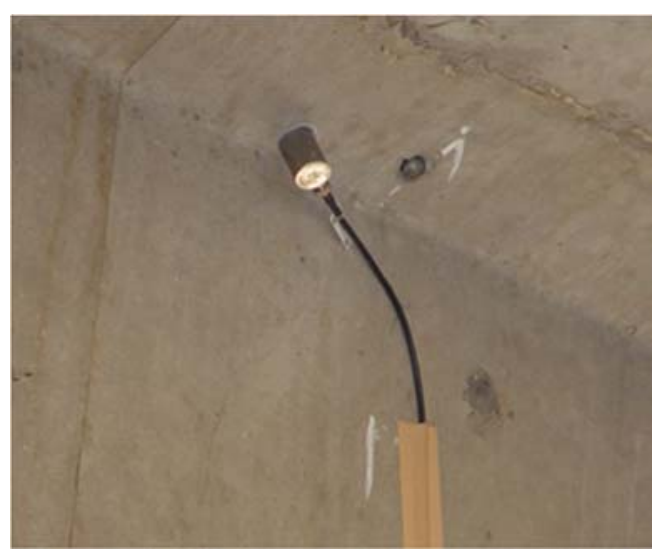

Figure 1.2. Acoustic emission sensor attached to a concrete girder (source: Physical Acoustics, 2018) 
This research focused on the use of Acoustic Emission (AE) technology as a potential Quality Assurance/Quality Control (QA/QC) procedure in source locating of AE events associated with cracking of prefabricated and pre-stressed reinforced concrete Northeast Extreme Tee (NEXT) beams and Northeast Bulb Tee (NEBT) girders during specific prefabrication processes including; detensioning and craned lifting from form beds and during transport to installation site. The evaluation of the efficacy of AE technology, as part of this study, began with laboratory proof of concept testing before moving on to field testing of the highly stressed end zone regions of prefabricated and pre-stressed reinforced concrete NEXT beams and NEBT girders. The end zone regions of the prefabricated and pre-stressed reinforced concrete NEXT beams and NEBT girders are the major regions of stress transfer from the pre-tensioning strands to the surrounding concrete, which is approximately 60 times the pre-tensioning strand diameter, per 5.11.4.1 of AASHTO Bridge Design Specifications (PCINE-14-ABC, 2014). The current state of practice for pre-cast manufacturers, is to use empirical data and a trial and error approach to reduce the development of end zone cracking while achieving the required girder load capacity, with minimal specific guidance from codes and standards. This has led to a variety of end zone reinforcement procedures for the reduction of end zone cracking, unique to each pre-cast manufacturer. In addition to limited guidance on the prevention of end zone cracking there is also limited guidance on the accept/reject criteria for end zone cracks. Instead decisions to reject or accept a girder based on end zone cracking are often subjective and based on the experience and knowledge of the pre-cast manufacturers and inspectors. A long-term goal of this line of research is to develop a set of reject/accept criteria based on quantitative structural and serviceability boundary conditions. 
NEXT beams and NEBT girders were of interest to this study and were chosen for testing based on their regular use as PBES in the northeastern United States where these elements and any cracking are subject to harsh and fluctuating weather along with the application of de-icing materials that can cause accelerated corrosion and degradation of concrete.

NEXT beams have a double-tee beams provide for rapid PBES construction similar to box and hollow-core profiles that but has the additional benefits of ease of inspection and no void space for water to accumulate (Tuan et al., 2004; Okumus et al., 2016; Arancibia and Okumus, 2017; Ronaki et al., 2017). NEXT beams also have an integral deck such that laying sections of NEXT beams together create a bridge deck and girder system that only needs a foundation and surface finishing (PCINE-14-ABC, 2014).

NEBT girders combine a single tee on top with a deep web connecting to a bulb section on the bottom. The evolution of designs of NEBT girders is toward deeper and more slender sections to allow for an increased number of pre-tensioning strands to induce larger amounts of pre-stress into the NEBT girders (Hasenkamp et al., 2008). This results in end zone cracking patterns similar to the NEXT beams. Although small end zone cracking may not make the beam structurally deficient, it can cause durability issues by allowing water and de-icing solutions to be in contact with the reinforcing steel or pretensioning strands, leading to corrosion and eventually to structural deficiencies.

The three major types of characteristic end zone cracking of NEXT beams and NEBT girders include; horizontal web cracking, inclined web cracking, and Y cracking. A typical cause of horizontal web cracking is eccentric loading. Pre-tensioning strand distributions are typical sources of inclined web cracking and Y cracking. Both horizontal 
and inclined web cracking are typically small enough that they close under service loading. Y cracking does not normally close under service loading and therefore has the greatest potential for durability issues (Okumus and Olivia, 2013). Examples of horizontal web cracks observed in this study are visible in Figure 1.3.

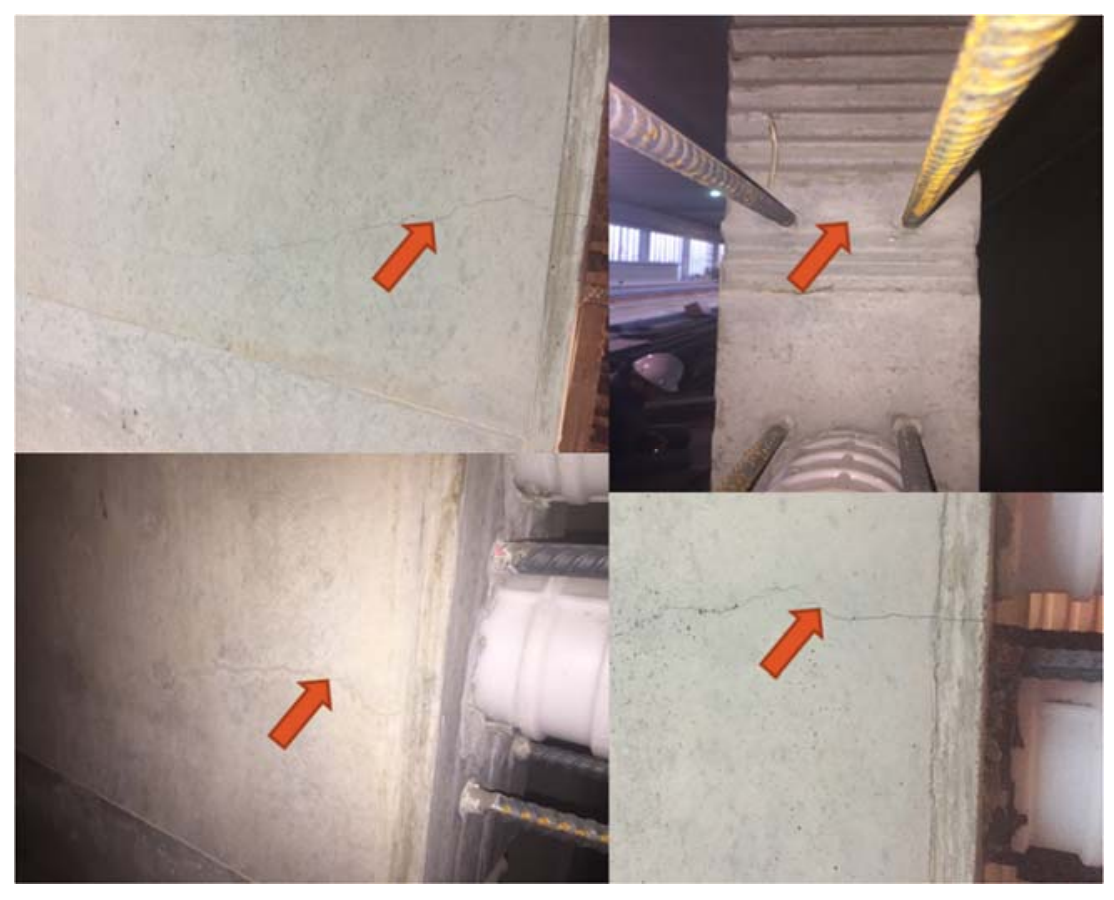

Figure 1.3. Recorded end zone cracking of NEBT girder

The most common end zone cracking control method is to alter the end zone reinforcing bar pattern. Typical end zone reinforcing steel patterns were developed based on experimental data, linear analytical studies, and finite element analysis and were mostly developed based on analyses of vertical flange cracking (Okumus and Olivia, 2013). While current end zone reinforcement pattern practices have mostly eliminated vertical flange cracking, the issues of horizontal, inclined and $\mathrm{Y}$ cracking of the web remain as challenges that require further research. 


\subsection{Objectives}

This research aimed to design and implement a reusable instrumentation system for evaluating the condition of structural elements typically used in the construction of transportation structures in the northeast United States. Quality control of the processes is an opportunity for improved final delivery of the product at reduced cost. Additionally, this research focused on developing reusable instrumentation for monitoring pre-stressed concrete girders during fabrication and transport, while also considering future research related to installation and initial traffic-bearing phases. The instrumentation should be reusable for multiple bridge projects.

The specific objectives of this research were to:

Objective 1. Perform tests on reinforced concrete beams under controlled laboratory conditions to establish the efficacy of AE technique in detecting cracking.

Objective 2. Perform field tests on prefabricated, pre-tensioned reinforced concrete NEXT and NEBT girders during detensioning, craned lifting, and transport.

Objective 3. Propose damage assessment techniques that could be utilized as a Quality Assurance/Quality Control (QA/QC) measure for prefabricated pre-stressed concrete girders used as Prefabricated Bridge Elements and Systems (PBES).

\subsection{Organization of the Thesis}

This chapter follows with Chapter 2 that presents a concise literature review on prefabricated and pre-stressed, reinforced concrete NEXT beams and NEBT girders, acoustic emission data collection techniques and methodologies, along with statistical and empirical methods for acoustic emission event source locating, differentiation, and damage assessment. 
Chapter 3 presents the selected acoustic emission instruments and rationale for selections. The chapter also presents details for the selected equipment and recommendations from the manufacturer. Additionally, Chapter 3 presents some initial and preliminary tests performed to verify the functionality of the acoustical emission monitoring equipment.

Chapter 4 describes the multiple laboratory tests on small reinforced concrete beam specimens performed to verify the performance of the selected AE equipment in detecting cracks in the beams.

Chapter 5 provides fabrication process observations, methodologies, data collected, and results of field testing on full-scale pre-stressed, reinforced concrete Northeast Extreme Tee (NEXT) beams and Northeast Bulb Tee (NEBT) girders. This chapter also describes some of the challenges with data collection and correlations established between beam features and clustering of acoustic emission events.

Chapter 6 describes the transport process observations, methodologies, data collected, and results of field testing on full-scale pre-stressed, reinforced concrete NEBT girders from J.P. Carrara and Sons, Inc. in Middlebury, VT to the I-91 bridge construction site located in Rockingham, VT. This chapter also describes some of the challenges with data collection and correlations established between travel conditions and $\mathrm{AE}$ event clustering as well as finite element stress modeling and AE event clustering.

Chapter 7 details current damage assessment techniques and procedures for reinforced concrete and discusses their relevance to the unique loading scenarios of fabrication and transport testing. Due to the general development of current damage assessment techniques with a cyclic loading regime; this chapter describes possible 
alterations of existing damage assessment tools to work with the unique loading conditions of fabrication and transport along with recommendations and hypotheses of new approaches for damage assessment in pre-stressed, reinforced concrete beams and girders.

Chapter 8 concludes this thesis with overall conclusions and recommendations along with proposed future work. 


\section{CHAPTER 2 BACKGROUND LITERATURE}

This literature review focuses on prefabricated and pre-tensioned reinforced concrete Northeast Bulb Tee (NEBT) girders and Northeast Extreme Tee (NEXT) beams, end-zone cracking, an overview of acoustic emission monitoring, acoustic emission sensor types, acoustic emission wave modes and propagation, acoustic emission source locating, acoustic emission source differentiation, and acoustic emission damage assessment.

\subsection{Pre-Tensioned and Post-Tensioned Reinforced Concrete}

Concrete technologies date back to antiquity and continue to advance to this day.

A significant modern development is composite technologies with the introduction of reinforcing and then pre-stressing to accommodate the inherently weak strength of concrete. The first patent filing for pre-stressing of concrete was in 1886 in San Francisco, CA (NJIT, 2018). There are three main types of structural elements used in modern construction; structural steel, reinforced concrete, and pre-stressed concrete. Figure 2.1 is a flow chart of the varieties of structural materials and specifically the varieties of prestressed concrete. 


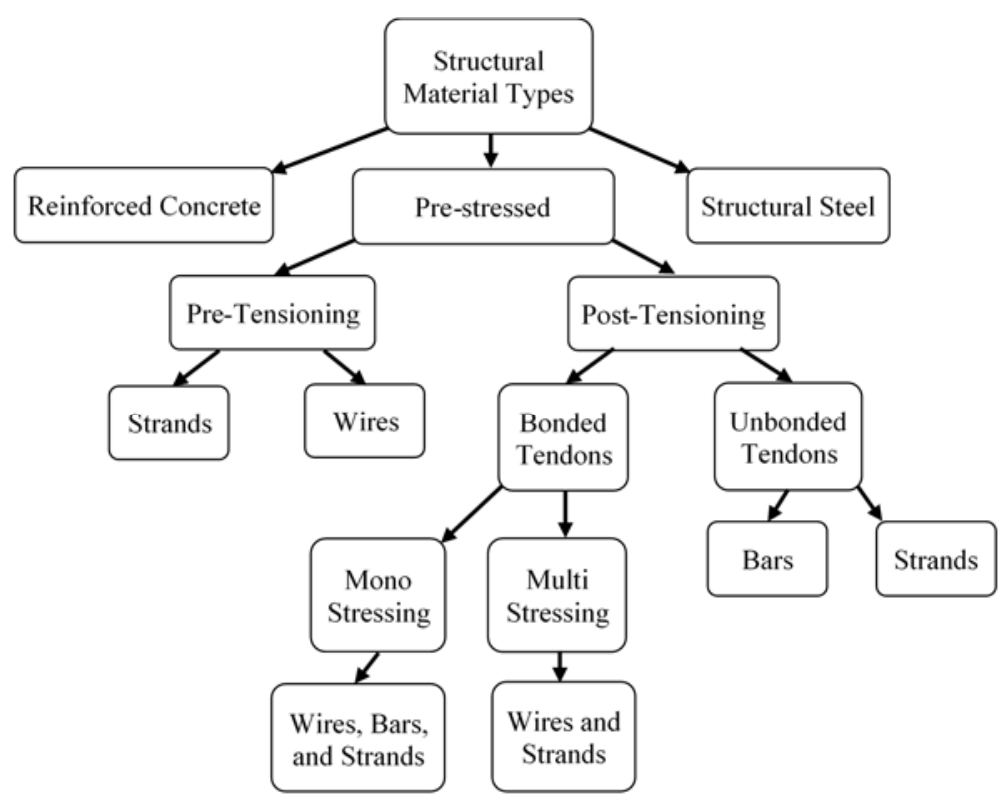

Figure 2.1. Structural material and pre-stressed concrete types flow chart (source: Steel Auto Industries, 2018)

Pre-stressed concrete uses either a pre-tensioning and/or post-tensioning technique to induce compression in the concrete element prior to service loading. The introduction of compressive stress prior to service loading counteracts some tensile loading which allows for the pre-stressed concrete to carry more tensile forces than non-pre-stressed concrete (Vejvoda, 2018). A schematic of the typical process for pre-tensioning and posttensioning of concrete appears in Figure 2.2. 


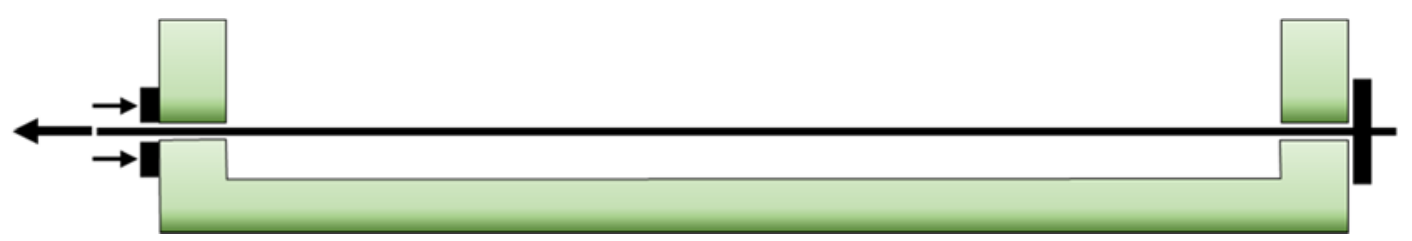

(a)

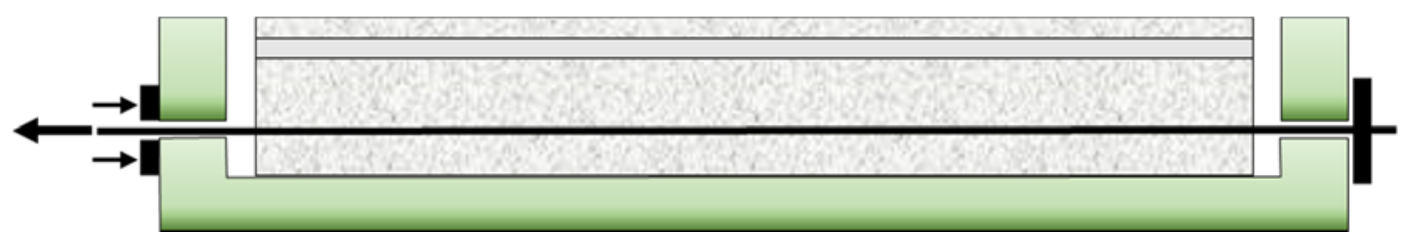

(b)

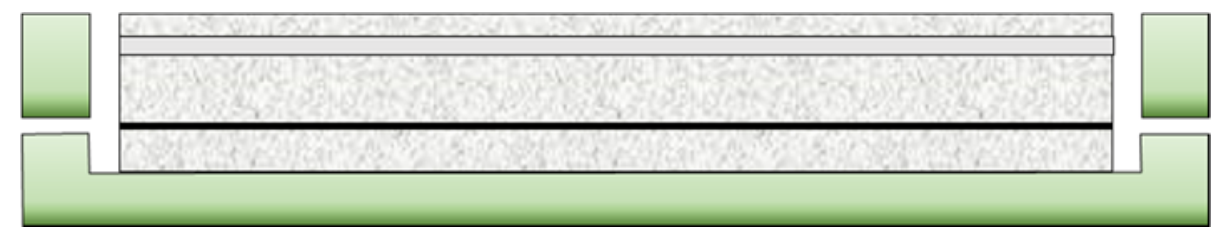

(c)

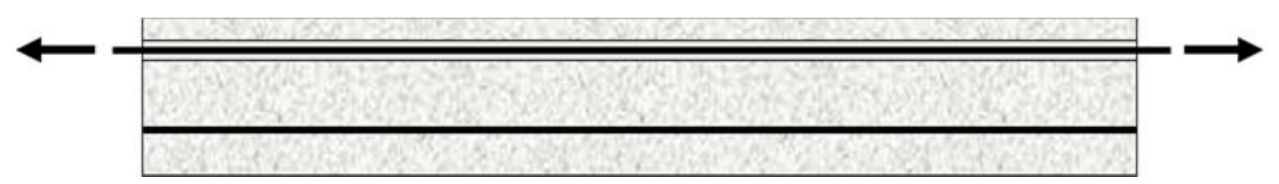

(d)

Figure 2.2. Pre-tensioned and post-tensioned concrete (a) tensioning of the pre-stressing strands against the end abutments, (b) casting of the reinforced concrete beam, (c) detensioning of the pretensioning strands, and (d) post-tensioning of the reinforced concrete beam

Typically, the pre-tensioning and post-tensioning tendons are steel but may also be made of various other materials such as nylon and fiberglass depending on the application. The pre-tensioning strands can be sleeved to control the spatial distribution of the prestressing, or not sleeved. A typical loading configuration uses hydraulic jacks pulling against a frame with bulkheads and deadman anchors to stretch the cable without applying any load to the concrete, steel reinforcing or formwork. Placing the concrete into the formwork encapsulates the pre-tensioning strands. Setup and curing of the concrete bonds the concrete to the pre-stressing strands. Once the concrete has cured to a sufficient 
compressive strength, torches cut the pre-tensioning strands which then compress the structural concrete element by a transfer of the tension with the shear developed on the outer surface of the strands.

Post-tensioning is an alternative method of pre-stressing concrete, often as a supplement to pre-tensioning. Post-tensioning offers a wide variety of advantages. Perhaps most importantly is the introduction of compressive force between individual structural elements to allow for continuity of longer spans. Post-tensioning strands may be either unbonded or bonded as seen in Figure 2.3.

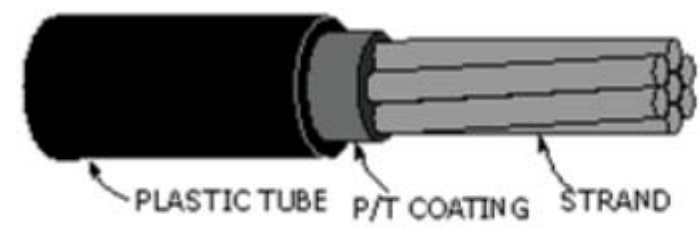

(a) PLASTIC SHEATH FILIED WITH GREASE CONTAIN ING

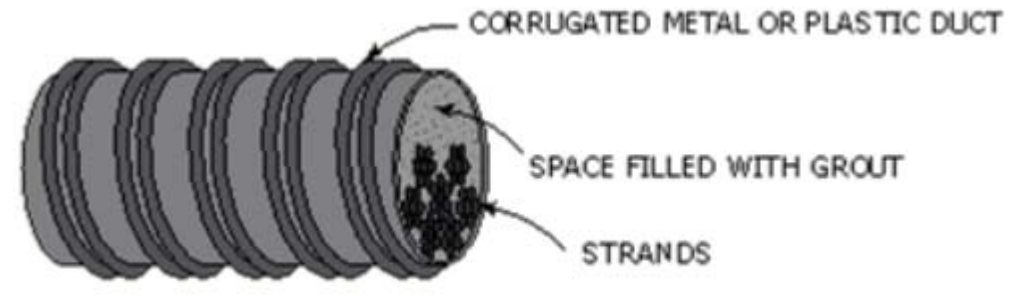

(b) CORRUGATED SHEATH WITH GROUTED STRANDS

Figure 2.3. Post-tensioning strands (typical) (a) unbonded post-tensioning strand and (b) bonded post-tensioning strand (source: Vejvoda, 2018)

Disadvantages of post-tensioning include the development of secondary moments when combined with pre-tensioning. Post-tensioning has the potential for pre-stress loss due to friction, wedge set, elastic shortening of concrete, concrete shrinkage, concrete creep, or steel relaxation (Vejvoda, 2018). 


\subsection{End-Zone Cracking}

Although there are standard designs for NEXT beams and NEBT girders, the designs continually evolve to meet project specific requirements that include deeper and more slender sections with increased pre-stressing which in some cases have been attributed to an increase in frequency and magnitude of end zone cracks; most notably in NEBT girders (Hasenkamp et al., 2008). The three most common occurrences of end zone cracking in NEBT girders are; horizontal web cracking, inclined web cracking, and Y cracking. The source of horizontal and inclined web cracking is typically eccentric loading

or pre-tensioning strand distribution. A primary source of $Y$ cracks is pre-tensioning strand distribution. Horizontal and inclined web cracks often close during in-service loading. Y cracks tend to not close and are of greater concern (Okumus and Olivia, 2013).

Current end zone cracking control methods for reinforcement bar pattern designs were developed with respect to vertical flange cracking and have been largely effective as vertical flange cracking is currently a rare occurrence (Okumus and Olivia, 2013). These same reinforcement bar patterns, however, have not been as effective in the reduction of horizontal web cracking, inclined web cracking, or Y cracking; supported by observations made during this study. The only cracks observed during this study were horizontal web cracks, Figure 2.4 . 


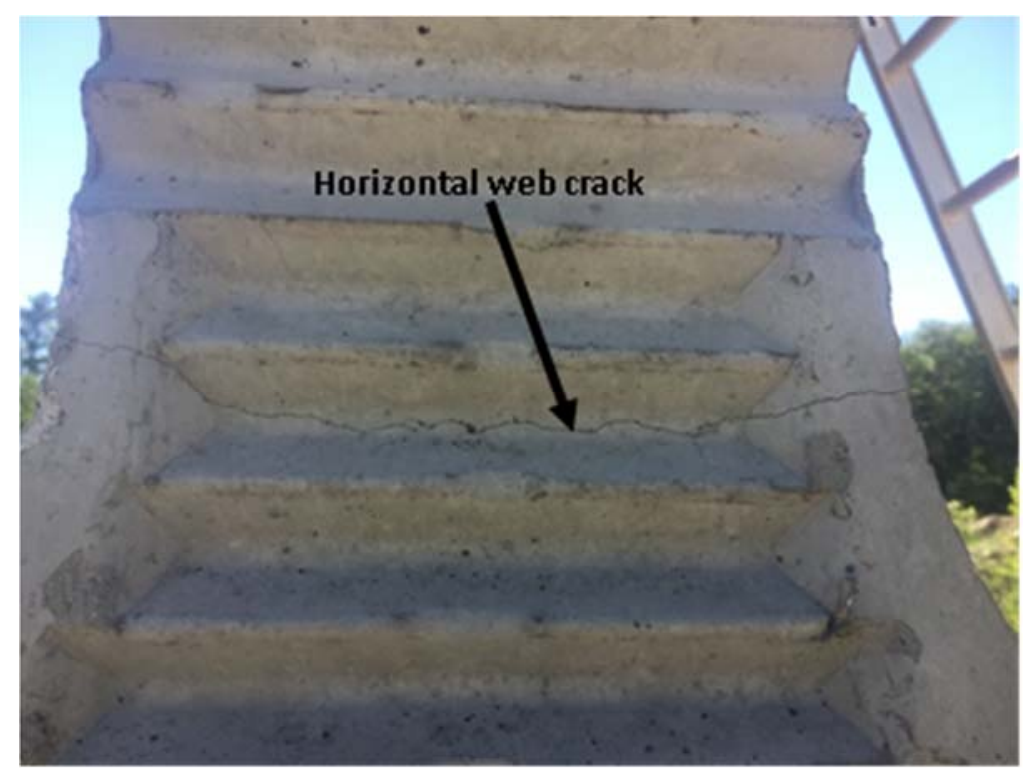

Figure 2.4. Horizontal end-zone web cracking of a NEBT girder

\subsection{Acoustic Emission Monitoring}

\subsubsection{Overview and History}

AE sensing by ear has been used for thousands of years with potters as early as 6,500 B.C. listening for the audible "tings" of crack nucleation during the kiln firing stage of creating their ancient ceramics (NDT Resource Center, 2018). These AE signals from the nucleation of a crack coupling with the air create the audible "tings" alerting the potter to a structural deficiency with their creation that could lead to its rejection. Modern AE technology appeared in the early 1950's with the completion of the "Results and Conclusions from Measurements of Sound in Metallic Materials under Tensile Stress," doctoral thesis of Joseph Kaiser of the Technical University Munich (TUM) (Tensi, 2004). Kaiser, in the 1960's, built equipment using piezo-crystal microphones that relayed signals to an oscilloscope where they could be recorded and analyzed. Later in 1961, Bradford Schofield coined the term "acoustic emission" (Grosse and Ohtsu, 2008). The first known practical application of AE technology was in 1964 with the testing and development of 
rocket motor casings (Kaphle, 2012a). Although early research and uses of AE technology focused on carbon steel and aluminum alloys (Tensi, 2004) it was not long until AE technology expanded for use with concrete and the U.S. Federal Highway Administration (FHWA) started research on the use of AE in bridge strength testing.

$\mathrm{An} \mathrm{AE}$ is a transient elastic wave produced by the release of stored elastic energy resulting in plastic deformations, termed as "damage". The release of stored elastic energy or redistribution of stresses can be caused by a wide variety of sources such as loading, pressure changes, temperature changes, or chemical reaction processes (NDT Resource Center, 2018). These AE event sources can be as small as micro and nano-scale cracking to catastrophic failures of full-scale bridge beams and girders. AE testing is considered a non-destructive test (NDT) method; although unlike many other NDT test methods, AE testing requires the material being monitored to crack or have some sort of plastic deformation. The need for a plastic deformation or cracking to occur for an AE to occur arguably makes $\mathrm{AE}$ testing a destructive instead of non-destructive test procedure. $\mathrm{AE}$ testing is also a passive technique that relies on a release of stored energy from the material instead of introducing energy into the material such as with ultrasonic testing.

There are many modern and commercially available AE detection systems that can record $\mathrm{AE}$ wave forms and record specific parameters related to the wave forms. These systems employ surface-mounted sensors that detect the propagating acoustical emission and convert the analog signal to a digital signal. Once in a digital format, it becomes convenient to filter signals with user identified inputs for setting thresholds and for preamplification of acoustical signals, amplitude thresholds, event duration thresholds, material wave mode velocities, etc. AE signals are typically weak. Some materials, 
including concrete, quickly attenuate with distance from the source emission location. It is common to use multiple amplifiers in a ganged configuration with a pre-amplifier and a main amplifier. Additional signal conditioning reduces background noise with a band-pass filter with a nominal pass band of several $\mathrm{kHz}$ to $1 \mathrm{MHz}$ (Grosse and Ohtsu, 2008). The AE signals from most civil infrastructure have an operating frequency range of $100 \mathrm{kHz}$ to $300 \mathrm{kHz}$ which is an achievable operating range of AE monitoring equipment (Kaphle, $2012 b)$.

\subsubsection{Acoustic Emission Sensors}

Although there are a variety of non-contact AE sensors such as fiber optic and laser interferometers, the optical AE sensors are limited in the physical area they are able to monitor as the distribution properties of light create the need to focus the light to a small area. Most AE sensors that operate with a surface contact configuration use the piezoelectric effect in lead zirconate titanate $(\mathrm{PZT})$ for transduction. The piezoelectric

effect is a reversible process. At a macroscopic scale piezoelectricity appears as the creation of a voltage across a solid as it deforms and vice versa the piezoelectric substance will deform in response to an applied voltage (Aysal, 2018). At the molecular scale piezoelectricity acts in anisotropic crystals in which crystal lattice deformations lead to the polarized movement of electric charge and vice versa. At the micro to nano scales, sintering-type manufacturing processes cause PZT to take on a polycrystalline structure with random polar orientations of the piezoelectricity as illustrated in Figure 2.5. 


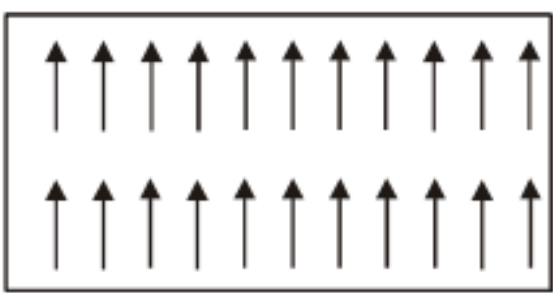

Mono-crystal with Single Polar Axis

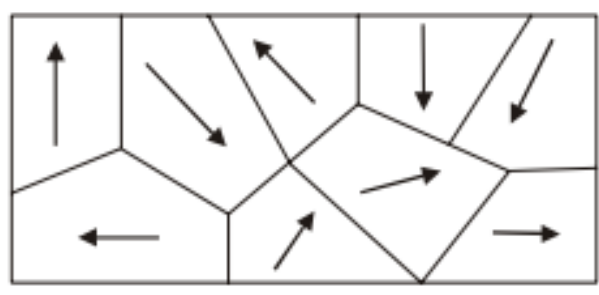

Polycrystal with Random Polar Axis

Figure 2.5. Mono-crystals vs. poly-crystals (source: Aysal, 2018)

Applying an electric field to the PZT polycrystal at a suitably elevated temperature biases the dipole molecules of the PZT to line up to some extent as illustrated in Figure 2.6 (Aysal, 2018).

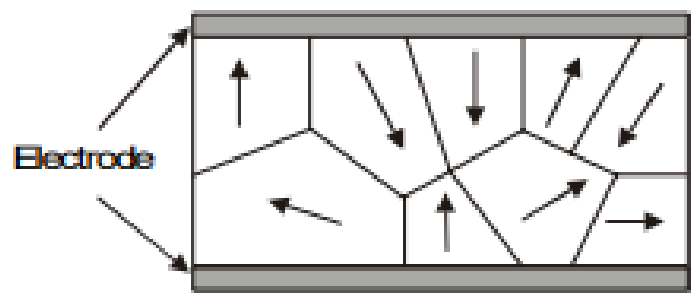

Random Dipole

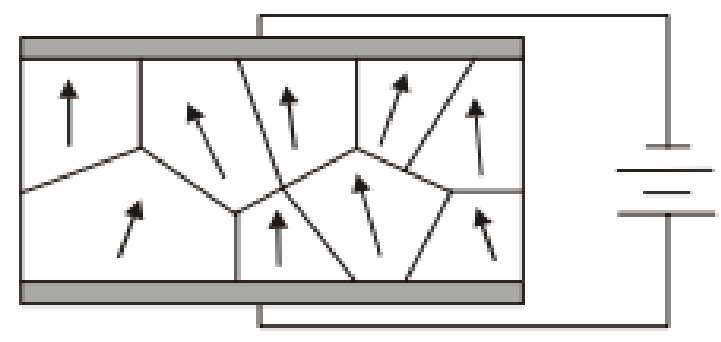

Polarization

Figure 2.6. Polarization of ceramic material to generate piezoelectric effect (source: Aysal, 2018)

The polycrystalline approach enables the manufacturing of PZT elements into various shapes and sizes to achieve different vibration modes and range of operating frequencies. Figure 2.7 shows the different piezoelectric responses to different loading scenarios with Figure 2.7.f being a common configuration for use in AE testing. 


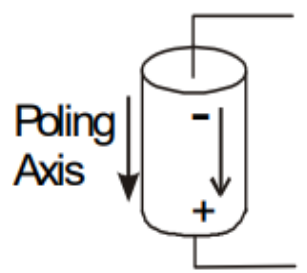

(a)

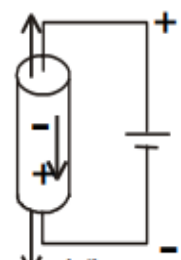

(d)

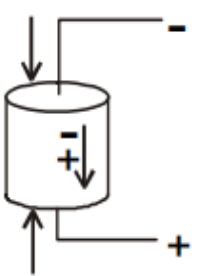

(b)

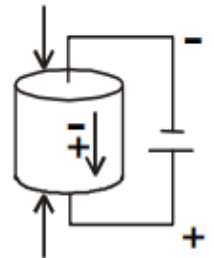

(e)

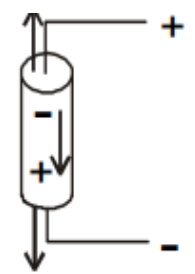

(c)

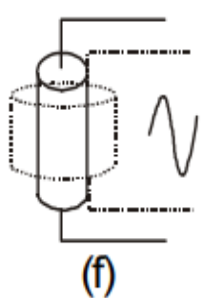

Figure 2.7. Piezoelectric effect under different circumstances (a) no stress or charge, (b) compression, (c) tension, (d) applied voltage opposite polarity, (e) applied voltage same polarity, and (f) applied AC signal (source: Aysal, 2018)

The surface-mounted AE sensor experiences the vibration of the AE which excites and deforms the piezoelectric element and produces a voltage. Specific to PZT elements; a $0.1 \%$ deformation generates a measurable piezoelectric response, often in the microvolt range (Krautkrämer et al., 1990). Since the mechanical deformations and piezoelectric transduction sensitivities are small, the initial analog signals require multiple layers of amplification during transmission and digitization. This overall process eventually converts the analog $\mathrm{AE}$ signal to a digital signal which can then be interpreted by the $\mathrm{AE}$ monitoring equipment and displayed to the end user. A typical surface mounted sensor is illustrated in Figure 2.8. 


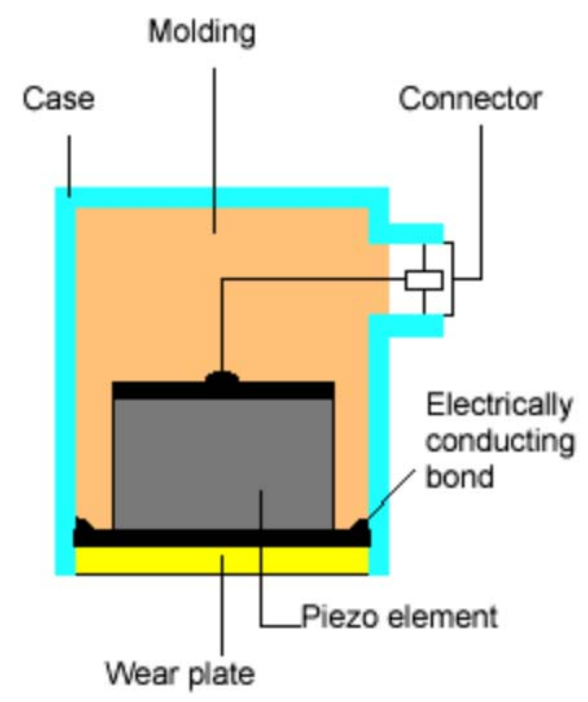

Figure 2.8. Typical piezoelectric sensor schematic (source: Vallen, 2009)

There are two primary variants of piezoelectric sensors; resonant and broadband. The main difference is the transfer of the $\mathrm{AE}$ signal to the piezoelectric element. In a resonant sensor, a small proof mass mechanically couples to the piezoelectric element through a flexible mount and vibrates freely around resonance in response to the AE signal. A broadband sensor has a stiff support connected to the piezoelectric element that directly applies deformations to the piezoelectric element. Each sensor has advantages and disadvantages which can make for best use scenarios for each sensor type. As illustrated in Figure 2.9, resonant sensors are more sensitive and better at detecting timing and event counts but can distort the recorded AE signal since the AE signal is transferred from the solid to the proof mass and then to the piezoelectric element. The broadband sensors are less sensitive but have a high-fidelity direct transduction of the AE signal to the piezoelectric element. Since with a broadband sensor there is not a proof mass but instead a stiff support, the sensor can be used in reverse as described previously where the 
application of an electric current of oscillating voltage to the piezoelectric element results in ultrasonic vibrations.

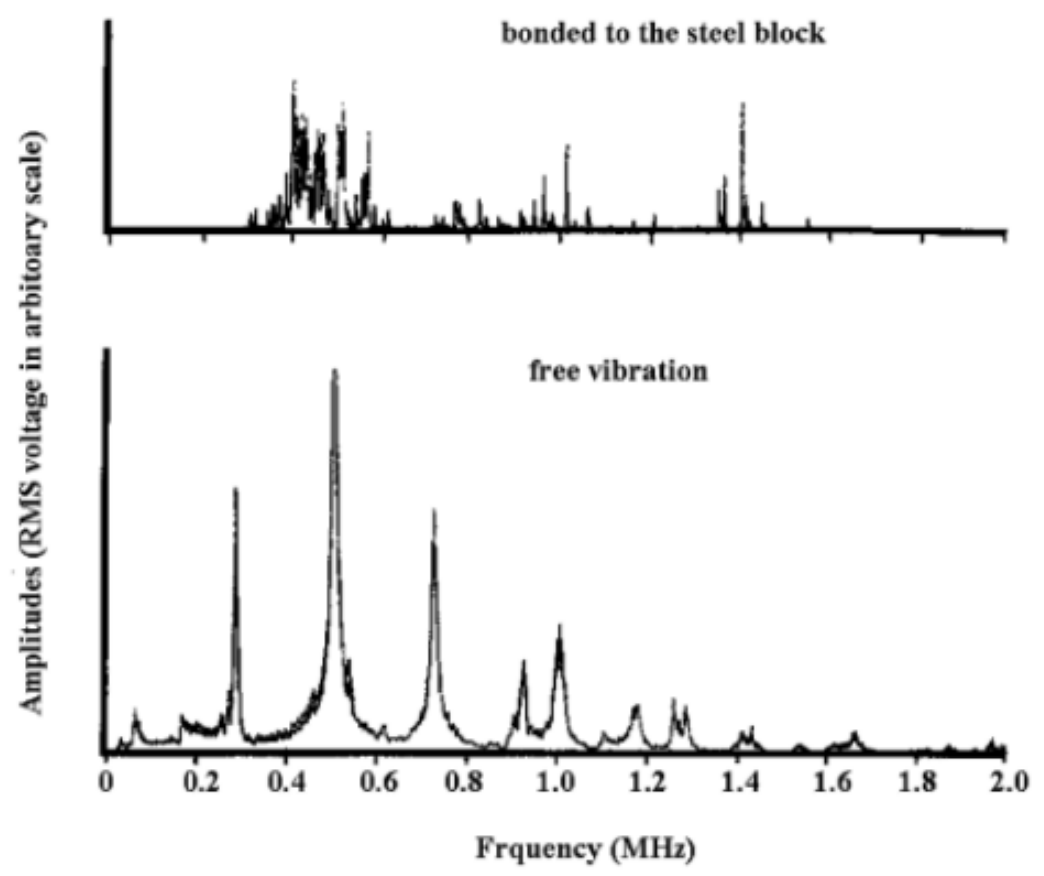

Figure 2.9. Broadband (top) vs. resonant (bottom) sensitivity comparison (source: Grosse and Ohtsu, 2008)

\subsubsection{Acoustic Emission Wave Modes and Propagation}

The nucleation of a crack, crack growth, rubbing, loading, and other irreversible deformative processes that emit an $\mathrm{AE}$ do so through multiple different wave modes. The three main wave modes measures for AE monitoring include; longitudinal waves (body wave/P-wave), transverse waves (shear wave/S-wave), and surface waves (Rayleigh wave). Longitudinal waves or P-waves are where particles oscillate in the direction of the wave propagation. Transverse waves or S-waves are where particles oscillate transverse to the direction of the wave propagation. P- and S-waves travel through bulk solids, with the P-waves having the higher velocity. Surface waves travel along the surface of a solid. Surface waves can result from P- and S- waves interacting at a surface (Kaphle, 2012a). 
The typical earthquake has $\mathrm{P}$-waves, $\mathrm{S}$-waves and surface waves, with the S-waves usually being the strongest and being the primary source of damage.

The AE waveform released from the source location propagates in all directions in a pulse-like manner. Anisotropy at the AE source can lead to a preferred directionality associated with the waveform. The pulses can be very short in time duration such as is the case with microcracking which releases $\mathrm{AE}$ signal pulses with durations lasting anywhere from a fraction of a microsecond to a few microseconds. The signal eventually measured by the $\mathrm{AE}$ sensor is not the same as that from the $\mathrm{AE}$ source. Extracting information for the measured signal requires additional signal conditioning, such as filtering, and postprocessing. The $\mathrm{AE}$ signal detected at the $\mathrm{AE}$ sensor is a combination of the initial $\mathrm{AE}$

signal, and reflected and refracted signals, background signals, and the coupling of different signals at the same phase.

\subsubsection{Acoustic Emission Source Locating}

The pulse-like transmission of AE signals from crack nucleation, crack growth, and other events emanate in all directions from the source point. Measuring the time of arrival (TOA) of a signal with an array of point sensors enables locating the emission source through triangulation, Figure 2.10. 


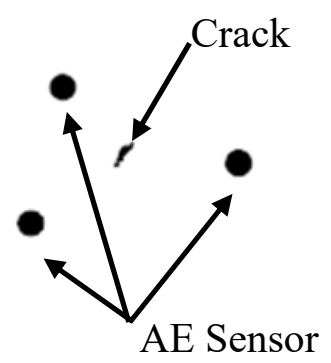

(a)

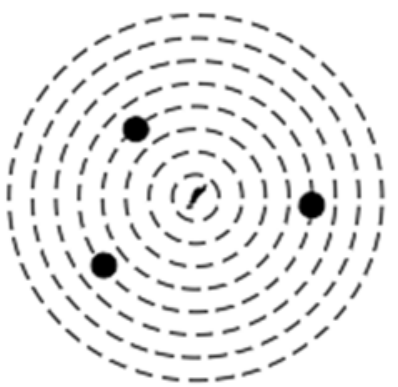

(b)

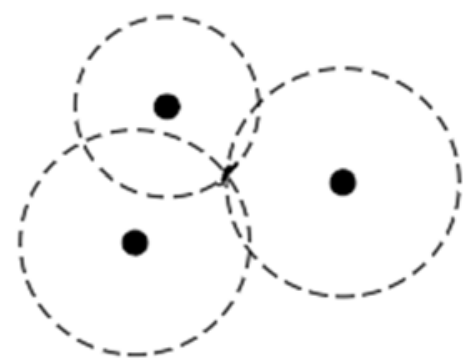

(c)

Figure 2.10. AE signal triangulation schematic (a) crack nucleation and AE sensors, (b) acoustical emission of transient elastic wave, and (c) AE source location by triangulation (source: Huston, 2010)

Locating an $\mathrm{AE}$ source with accuracy requires recording $\mathrm{AE}$ signals with microsecond precision. The accuracy of the source location also depends on the wave propagation velocities of the solid. A concern with concrete is the attenuative nature of the material in the range of $40 \mathrm{~dB} /$ meter and scatter of the AE signal due to wave interaction with natural air voids or existing cracks. Nonhomogeneous wave speeds due to reinforcing, differing states of cure and damage can all contribute to distorting the wave propagation and confounding source location estimates.

\subsubsection{Acoustic Emission Source Differentiation}

The AE signals carry additional information beyond TOA, largely in the detailed shape of the waveform. Such information correlates to the details of the AE source and the path traveled. There are two main types of AE source differentiation techniques (Kaphle, 2012b). The first is a feature-based analysis where an analysis of the specific waveform extracts and records a set of features, also known as parameters, describing the wave form. Typical features include amplitude, duration, counts-to-peak, and peak frequency as illustrated in Figure 2.11. Subsequent processing of waveform features extracts information. 


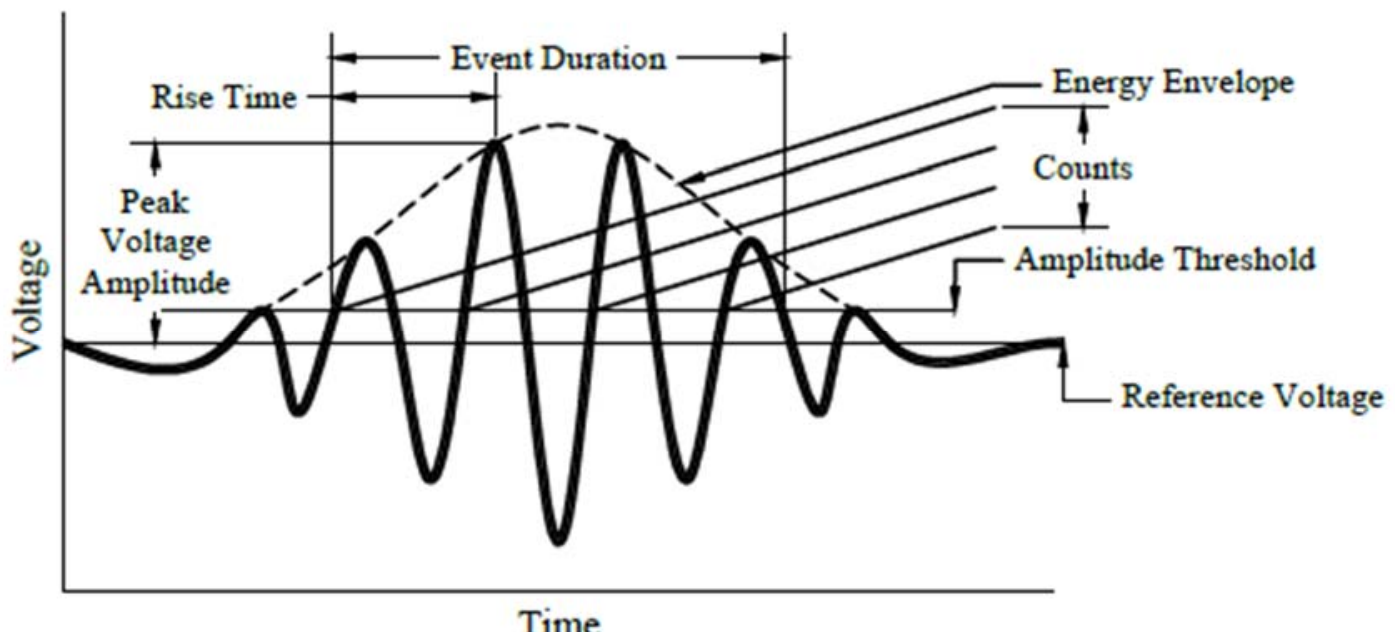

Figure 2.11. Parametric analysis of typical measured waveform features

The second $\mathrm{AE}$ source differentiation method begins by recording entire $\mathrm{AE}$ waveforms for a more detailed post-data collection analysis. Success requires sampling at a sufficiently high frequency to avoid Nyquist and related under-sampling problems. This creates a far larger volume of data (Kaphle, 2012a). Two primary emission types are burst and continuous, Figure 2.12. A burst emission is typically the result of crack nucleation or growth with a characteristic short-duration burst of waveform wiggling. Continuous emissions produce a steady stream of merged bursts that appear as stationary noise. The kinetic nature of all solids produces a low-level of continuous emissions. Many macroscopic processes also produce continuous emissions. Selecting the appropriate amplitude filter can pre-filter these non-interesting continuous emissions prior to postprocessing. 


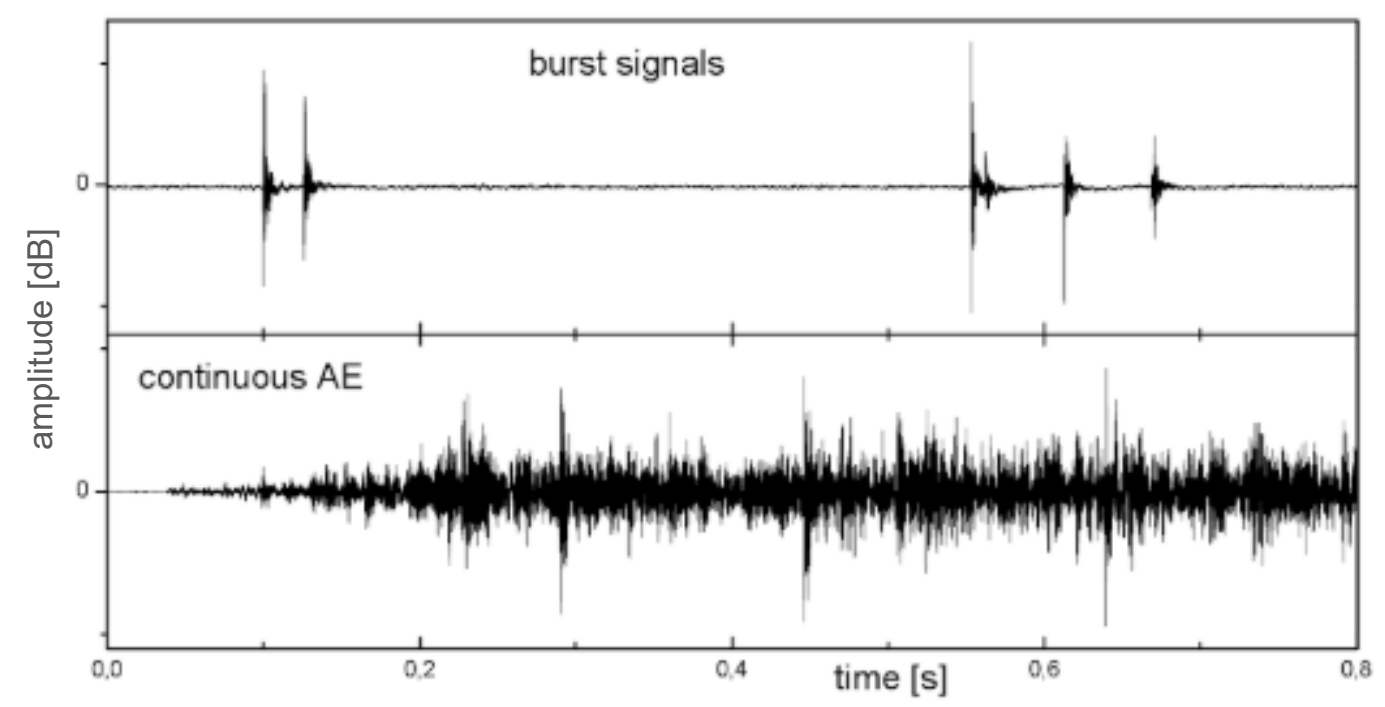

Figure 2.12. Burst emission (top) vs. continuous emission (bottom) (source: Grosse and Ohtsu, 2008)

Multi-sensor spatial filters provide additional capabilities, such as the identification and rejection of anomalous situations, such as multiple simultaneous events, remote events outside the region of interest and secondary reflections from surface boundaries and the rubbing of newly formed cracks (Kaphle, 2012b).

A study by ElBatanouney et al. (2014) used an amplitude and duration filter to eliminate $\mathrm{AE}$ events not correlated to crack formation during a laboratory test of cyclically loading a reinforced concrete beam to failure. Figure 2.13 shows how a careful choice of filter settings can reduce AE event location estimates from a diffuse swath of points centered on the cracks, Figure 2.13.b, to a smaller set of points that provide a superior alignment with the observed cracks, Figure 2.13.c. 


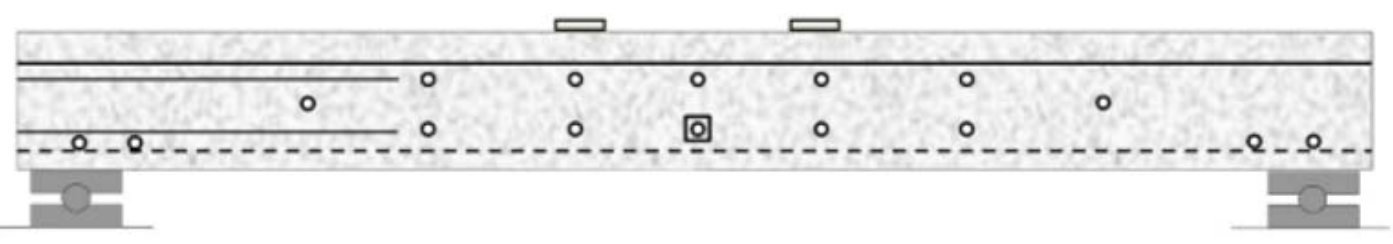

(a)

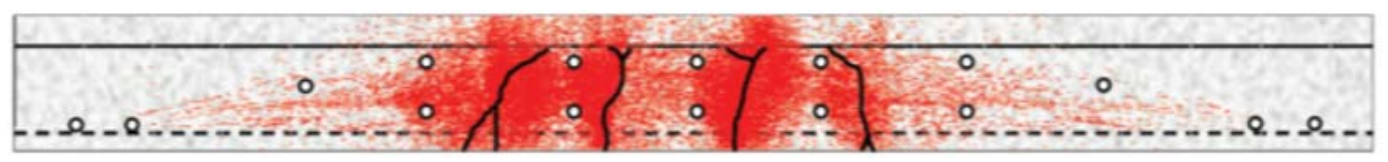

(b)

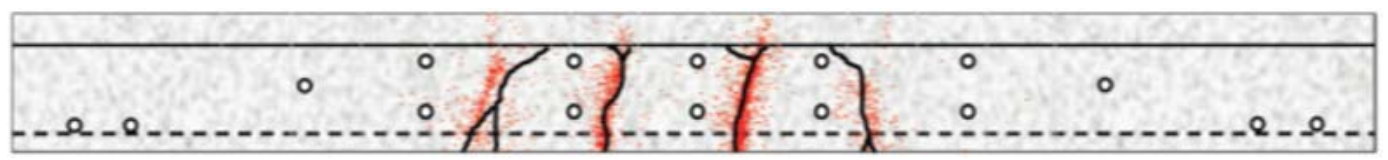

(c)

Figure 2.13. AE event amplitude and duration filtering for crack correlation (a) laboratory beam and $\mathrm{AE}$ sensor array, (b) unfiltered $\mathrm{AE}$ event location, and (c) filtered $\mathrm{AE}$ event locations correlated to observed cracking (source: EIBatanouney et al., 2014)

\subsubsection{Acoustic Emission Damage Assessment}

Damage assessments and quantification can account for the stress level at which

AE events occur, peaks in amplitude of AE events, number of AE events, spatial clustering of AE events, and rate of accumulation of AE events (Arches, 2009). The technical literature contains multiple published damage assessment techniques, with the majority looking at conventional non-pre-stressed reinforced concrete. Additionally, most of the available damage assessment methods use cyclical loading scenarios that lend themselves for monitoring of bridges that experience cyclical loading and unloading from traffic. Some of the most common damage assessment techniques for reinforced concrete are: 1 . Felicity ratio; 2. Parametric analysis; 3. Load-Calm ratio; 4. B-value analysis; and 5. Frequency analysis. These damage assessment techniques and quantification metrics all use data obtained from the recorded $\mathrm{AE}$ waveform or parametric data representative of the 
$\mathrm{AE}$ waveform to relate the $\mathrm{AE}$ event with a crack event or to relate the $\mathrm{AE}$ event to a severity of damage.

\subsubsection{Felicity Ratio}

The basis of the Felicity ratio is the load history dependent Kaiser effect. The test protocol uses a sequence of loading and unloading cycles with an amplitude that increases at each cycle. The Kaiser effect is where no AE events occur until the load levels exceed the previous maximum loads. The Felicity effect is the opposite case where AE events occur at load levels below the previous maximum loads. This indicates the occurrence of damage.

$$
\text { Felicity Ratio }=\frac{\text { Load at AE restart }}{\text { Previously applied maximum load }}
$$

A Felicity ratio of 1.0 or greater indicates no damage, whereas, a Felicity ratio of less than 1.0 indicates damage. The lower the Felicity ratio the greater the severity of damage. The Felicity ratio concept is illustrated in Figure 2.14. 


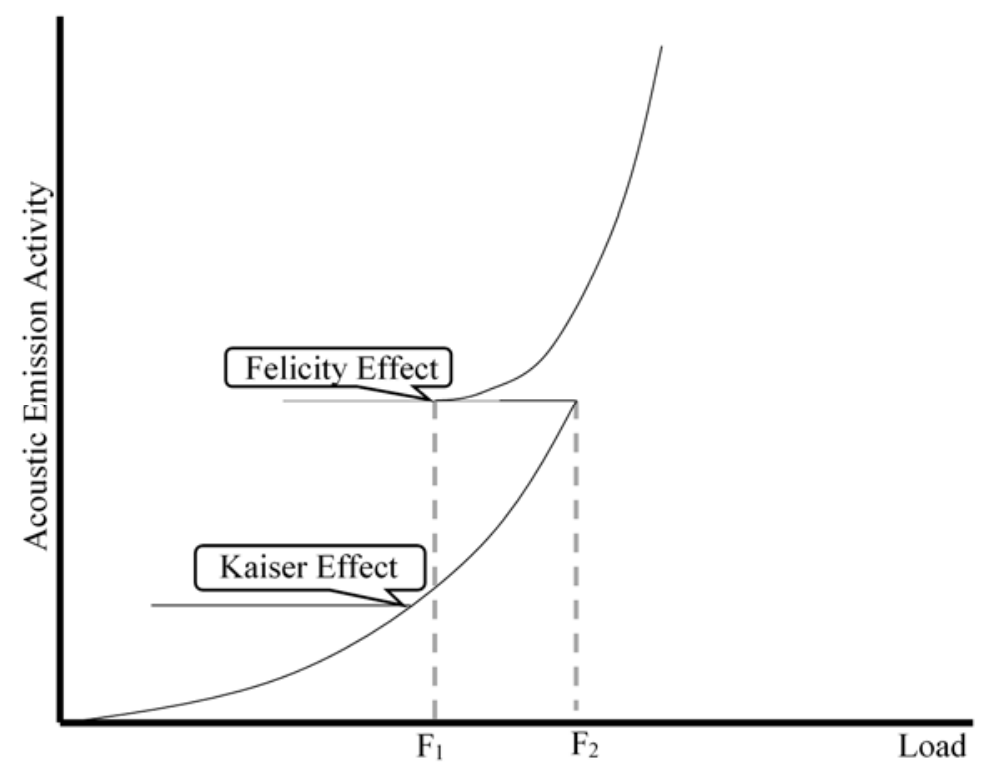

Figure 2.14 Felicity effect and ratio illustration (typical) (source: Arches, 2009)

\subsubsection{Parametric Analysis}

A parametric analysis uses basic parameters determined from the $\mathrm{AE}$ event waveform and observed damage features such as cracking to establish correlations between AE event parameters and damage. An example of a typical correlation is plotting the RA values (rise time/peak amplitude) versus the average frequency (kHz). AE events above the $y=1 / 10 x$ boundary indicates tensile cracking, whereas, AE events below the $y=1 / 10 x$ boundary indicate other types of cracking (Arches, 2009).

\subsubsection{Load-Calm Ratio}

The Load-Calm ratio damage assessment technique plots the Load ratio and Calm ratio of a loading-unloading cycle to classify the $\mathrm{AE}$ events as; minor damage, intermediate damage, or heavy damage. The Load ratio is the same as the Felicity ratio. The Calm ratio is the ratio of $\mathrm{AE}$ activity during the unloading process compared to the $\mathrm{AE}$ activity during 
the last loading cycle. Plotting the Load ratio versus the Calm ratio can then indicate minor, intermediate, or heavy damage in Figure 2.15.

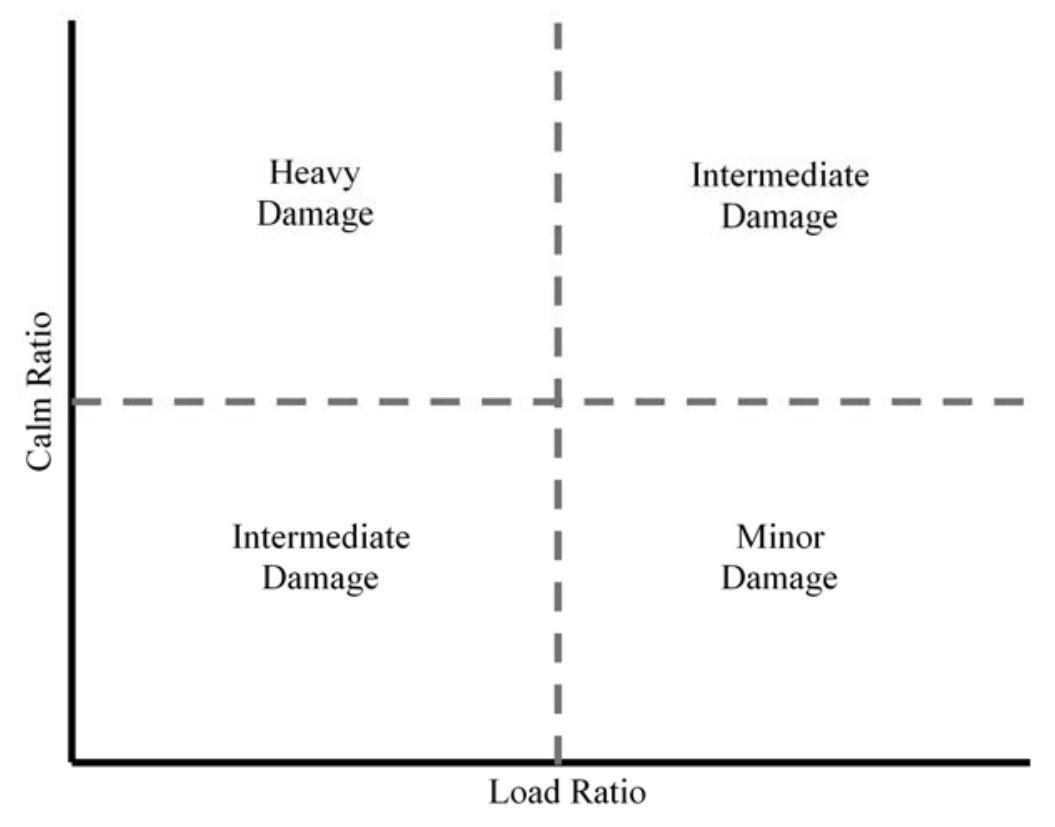

Figure 2.15 Load-calm ratio illustration (typical) (source: Arches, 2009)

\subsubsection{B-value Analysis}

The b-value analysis is a statistical regression of the AE event peak amplitudes that requires a complete loading-unloading cycle for calculation. The basis of the b-value analysis are from the seismic Gutenberg-Richter formula:

$$
\begin{gathered}
\log _{10} N=a-b^{\prime}\left(A_{d B}\right) \\
b-\text { value }=20 b^{\prime}
\end{gathered}
$$

where,

$\mathrm{N}=$ number of $\mathrm{AE}$ events with amplitude above $\mathrm{AdB}$

$\mathrm{AdB}_{\mathrm{dB}}=\mathrm{AE}$ signal amplitude 
$\mathrm{a}=$ empirical constant (background noise)

$\mathrm{b}^{\prime}=$ empirical constant

An abrupt decrease of the calculated b-value indicates the occurrence of damage. This method is sensitive to the attenuative nature of concrete. Appropriate sensor placement is critical for accurate results. The sensor array deployment must be near to or encompass the area of damage. The sensor spacing must be close enough to register $\mathrm{AE}$ events accurately. A nominal spacing of two meters or less is usually considered appropriate.

\subsubsection{Frequency Analysis}

The frequency analysis uses a basic flow chart, Table 2.1, to categorize concrete damage based on frequency and energy of the AE event. This method is specific to reinforced concrete and has a high potential to mis- categorize damage if the sensor placement is not close enough to accurately read AE events before severe attenuation by the concrete.

Table 2.1. Frequency analysis flow chart (source: Arches, 2009)

\begin{tabular}{|c|c|c|c|}
\hline \multirow{4}{*}{$\begin{array}{c}\text { AE } \\
\text { Source }\end{array}$} & $\begin{array}{c}\text { High } \\
\text { frequency }\end{array}$ & High energy & $\begin{array}{c}\text { Steel wire breaking or stress } \\
\text { corrosion cracking }\end{array}$ \\
\cline { 2 - 4 } & $\begin{array}{c}\text { Medium } \\
\text { frequency }\end{array}$ & Low energy & Steel corrosion \\
\cline { 2 - 4 } & $\begin{array}{c}\text { Low } \\
\text { frequency }\end{array}$ & $\begin{array}{c}\text { High energy } \\
\text { Changes in nonlinear } \\
\text { acoustic behavior of concrete }\end{array}$ & Structure damage under loading \\
\hline
\end{tabular}




\section{CHAPTER 3 DETAILED DESIGN OF INSTRUMENT PACKAGE}

\subsection{Introduction}

The research employed Mistras acoustic transducers, data acquisition set up and data processing software, which are described here. Some initial testing provided initial checks on the system, which are also described in this chapter. The field testing required some modifications to the system, which are described in later chapters on field testing.

\subsection{Instrumentation and Data Acquisition}

The main components of the system include: Sensor Highway III data acquisition system, eight PK6I $60 \mathrm{kHz}$ resonant acoustic sensors, 30 meters of additional signal cable, 2-D locations software, and 3-D locations systems. These are detailed in Table 3.1 and described further in subsequent sections.

Table 3.1. List of Mistras AEwin ${ }^{\text {TM }}$ Sensor Highway III System and Parts

\begin{tabular}{|c|l|c|}
\hline Item & \multicolumn{1}{|c|}{ Model and Description } & Quantity \\
\hline 1 & $\begin{array}{l}\text { PN\# SHIII-8, SHIII-8, Smart Remote Monitoring, 8-channel Sensor } \\
\text { Highway III system is a full, stand-Alone AE system for unattended } \\
\text { monitoring in outdoor environments. System Includes; Outdoor } \\
\text { weatherproof case (18" x 12" x 6"), one 8-channel AE board, 8 } \\
\text { single-ended parametric inputs, remote reboot, Windows 7 } \\
\text { Operating system, AEwin installed and licensed for 8 channels, } \\
\text { Ethernet connectivity to a factory network or Internet, 110/220VAC } \\
\text { or 9 - 28 VDC power at 15 watts. }\end{array}$ & $\begin{array}{c}\text { PN\# PK6I-5015, PK6I, Low Power Sensor, 60 kHz with Integral } \\
\text { Preamp and SMA Connector for Sensor Highway }\end{array}$ \\
\hline 3 & $\begin{array}{l}\text { PN\# 1234-4002-30, 1234-SMA/BNC-30, Signal Cable, RG58 SMA- } \\
\text { BNC 30 Meters }\end{array}$ & 8 \\
\hline 4 & $\begin{array}{l}\text { PN\# 9380-7003-2, AE-WIN 2D-LOC, FULL PLANAR, 2 } \\
\text { DIMENSION LOCATION }\end{array}$ & 1 \\
\hline 5 & $\begin{array}{l}\text { PN\# 9380-7003-9, AE WIN 3D-LOC, 3 DIMENSIONAL } \\
\text { LOCATION SOFTWARE OPT }\end{array}$ & 1 \\
\hline
\end{tabular}




\subsubsection{Power and Data Acquisition}

The Sensor Highway III, Figure 3.1, fits primarily in a NEMA 4 rated steel electrical box, with cables extending to the transducers, controlling computer and power supply. This system can operate and collect data from up to 32 acoustic emission sensors and other voltage-type transducers. The Sensor Highway III unit used as part of this research was equipped with one 8-channel AE board and employed 8 low voltage PK6I resonate AE sensors. The unit is powered by a standard wall plug that has been modified to connect to two 12-volt motor cycle batteries wired in series with a 3-amp fuse and switch.

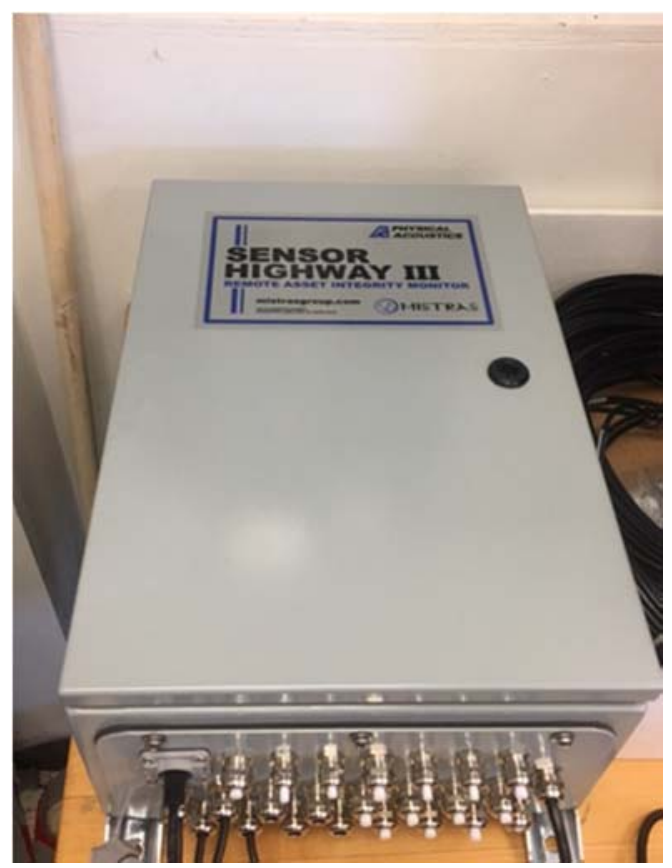

(a)

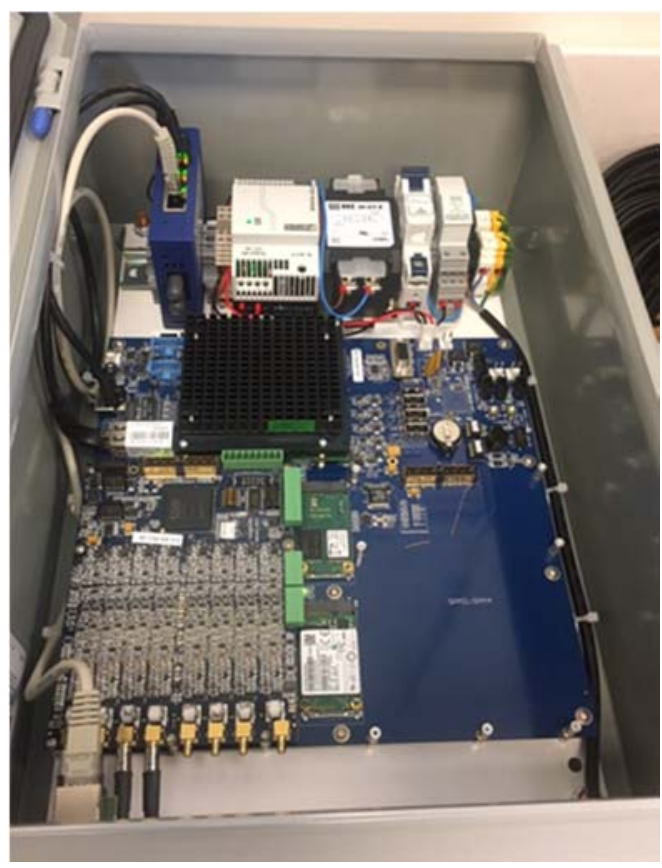

(b)

Figure 3.1. Sensor Highway III instrument box (a) lid closed and (b) lid open

\subsubsection{Acoustic Transducers}

This study employed a transducer array consisting of eight PK6I sensors configured and operated as per manufacturers' recommendations and ASTM standards (ASTM 
E1316-18a, 2018; ASTM E3100-17, 2017; Physical Acoustics, 2018). The PK6I sensor (Figure 3.2) is a medium-frequency, resonant AE sensor with an integral, ultra-low noise, low-power, filtered $26 \mathrm{~dB}$ preamplifier, which can drive up to 200 meters of cable and operates at $60 \mathrm{kHz}$ frequency (Physical Acoustics, 2018).

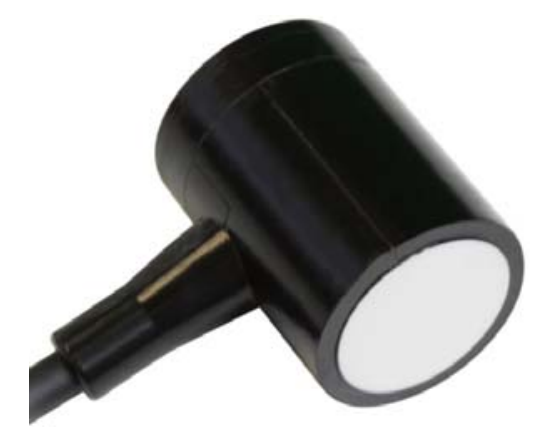

Figure 3.2. Acoustic emission low power, $60 \mathrm{kHz}$ PK6I sensor with integral preamplifier (source: Physical Acoustics, 2018)

\subsubsection{Software}

The data acquisition software includes both 2-dimensional (2-D) locating and 3dimensional (3-D) locating along with continuous waveform stream or parametric acquisition. The software requires user-defined input parameters to define the material and material properties such as; dimensions, sensor placement locations, data acquisition thresholds and filters, along with attenuation curves and material waveform velocities. It is important to note that the software only allows for homogeneous material properties and simple geometries. This becomes a challenge with future field testing of beams and girders that are made of nonhomogeneous materials and are of complex geometries. It is also important to note that the $\mathrm{AE}$ source locations software is a pseudo black box that relies on both time of signal arrival and waveform parameters but the exact algorithm is proprietary, and therefore, its accuracy can only be validated through pencil lead break tests described in Section 3.3. 


\subsubsection{Transducer Mounting}

The initial method of attaching the transducers to the concrete slab was to use a heavy silicone vacuum grease. This grease is remarkably sticky, but nonetheless did not provide a consistent and robust attachment to the concrete, largely due to the porous and rough nature of the surface. A mechanism to both maintain connection between the $\mathrm{AE}$ sensor wear-plates and the concrete as well as to protect the AE sensors from damage was needed. To alleviate these concerns, two types of metal armor/fastening devices were fabricated. The first attempt of fabricating metal armor/fastener included cut sections of square hollow stock large enough to accommodate the AE sensor (Figure 3.3a). A hole was then drilled and tapped on one side to allow for the installation of a set screw to hold the AE sensor in place. The metal armor/fasteners were then epoxied with two-part 5minute cure epoxy to the concrete surface. This design succeeded in both protecting the AE sensors during testing and maintaining contact to the concrete surface but there was concern that the metal between the AE sensor wear plate and the concrete surface could distort the AE signals. For this reason, a new approach was used; U-shaped brackets with two ears on each side to epoxy to the concrete surface were fabricated from metal sheet stock (Figure 3.3b). Again, these U-shaped brackets had a hole drilled and tapped for a set screw to pressure the AE sensor against the concrete surface. 


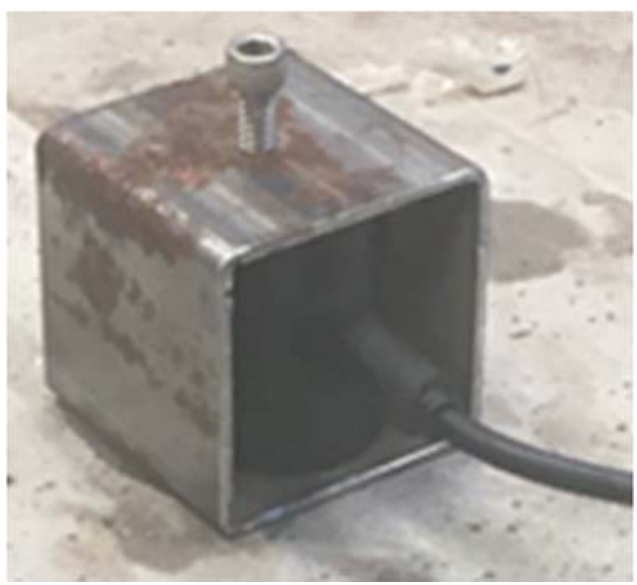

(a)

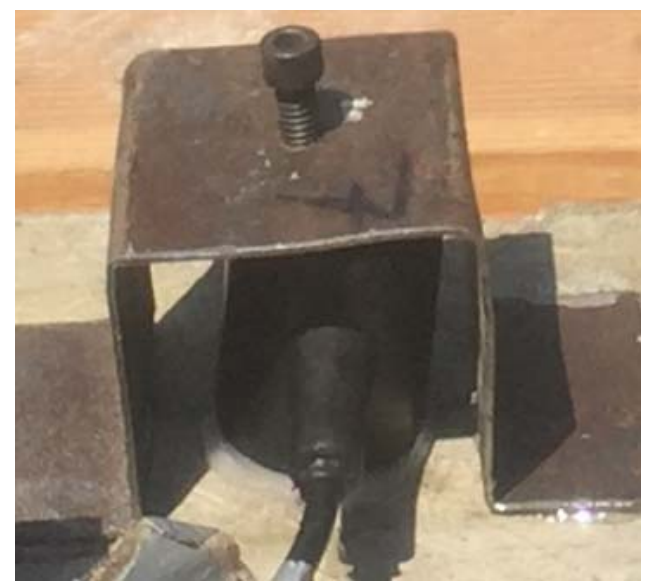

(b)

Figure 3.3. AE sensor housing mount (a) hollow square stock AE sensor mount (b) U-shaped bracket AE sensor mount

\subsection{Initial Testing and Triangulation Tests}

The initial tests assessed the ability to measure acoustic emission signals produced on concrete slabs in the laboratory. Two standard nondestructive methods of exciting the structure are a pencil lead break and impact from a dropped steel ball. Since the pencil lead break is a standard procedure and can be easily controlled and replicated, it was used to verify the accuracy of both the 2-D and 3-D locating software as part of the Mistras AEwin ${ }^{\mathrm{TM}}$ Sensor Highway III system. A relatively small reinforced concrete block 16.5inch x 9.75-inch x 5.5-inch (419 $\mathrm{mm}$ x $248 \mathrm{~mm}$ x $140 \mathrm{~mm})$ was cast and cured for 7 days in a custom walk-in Darwin Chamber $^{\odot}$ (Figure 3.4). 


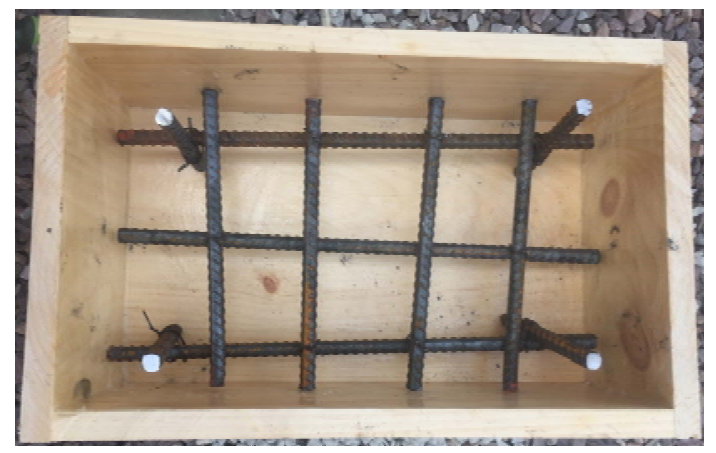

(a)

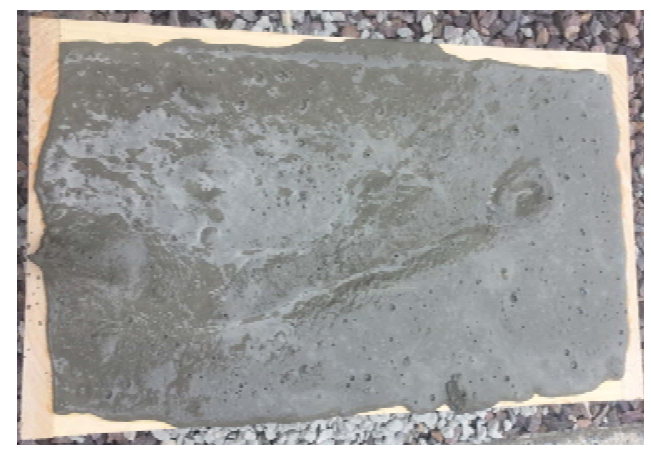

(b)

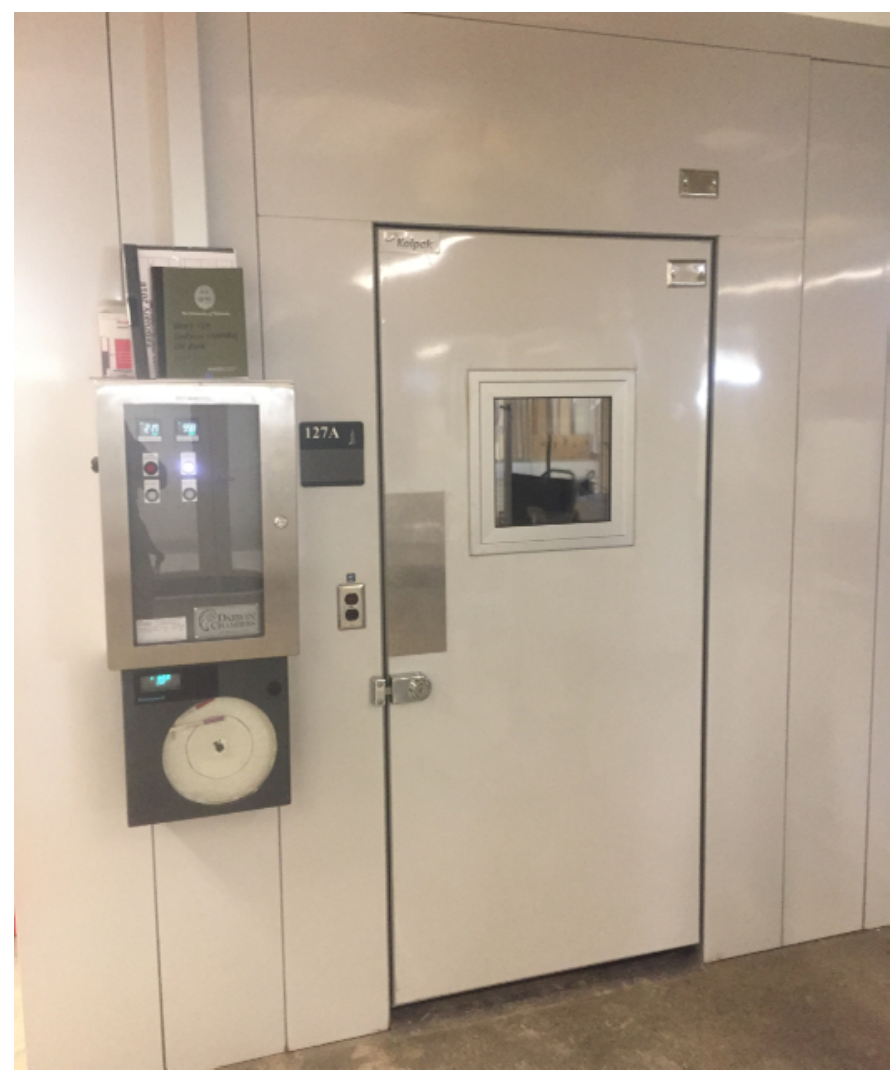

(c)

Figure 3.4. Fabrication of pencil lead break beam (a) reinforcing bar pattern, (b) freshly vibrated wet concrete in mold, and (c) Darwin Chamber $^{\mathfrak{O}}$ used for curing 


\subsubsection{2-D Pencil Lead Break Testing}

2-D pencil lead break tests performed on the relatively small concrete block indicated fairly accurate AE source location in the $x-y$ plane but lacked accuracy in distinguishing the depth of the AE source as illustrated in Figure 3.5.

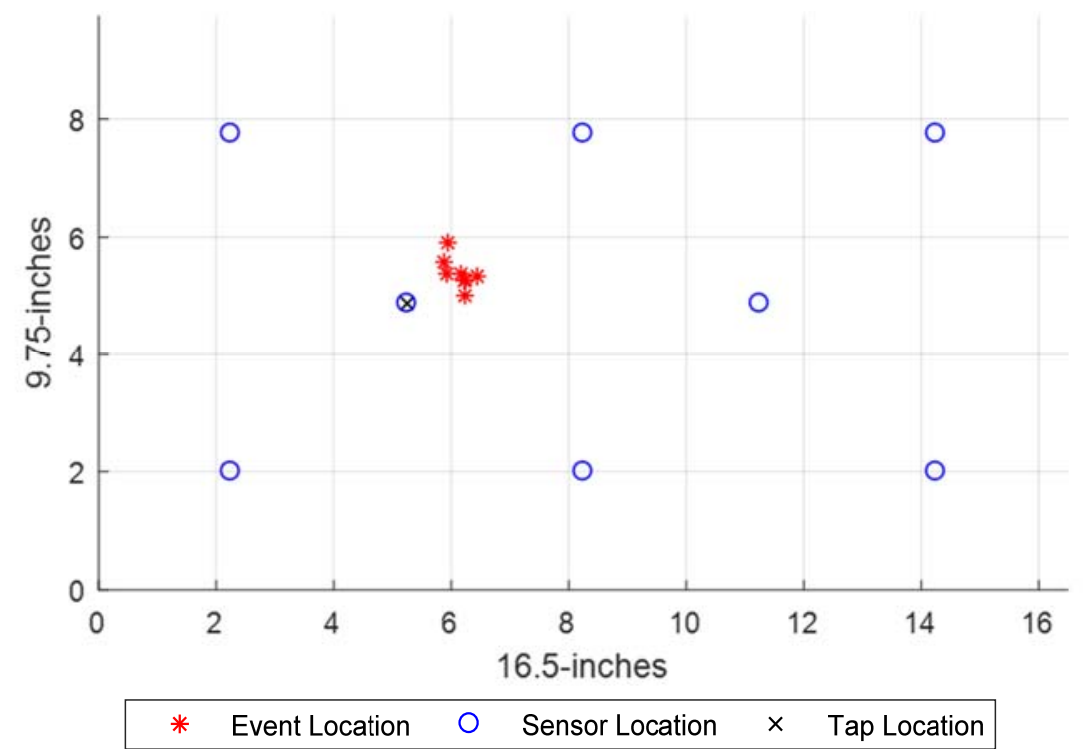

(a)

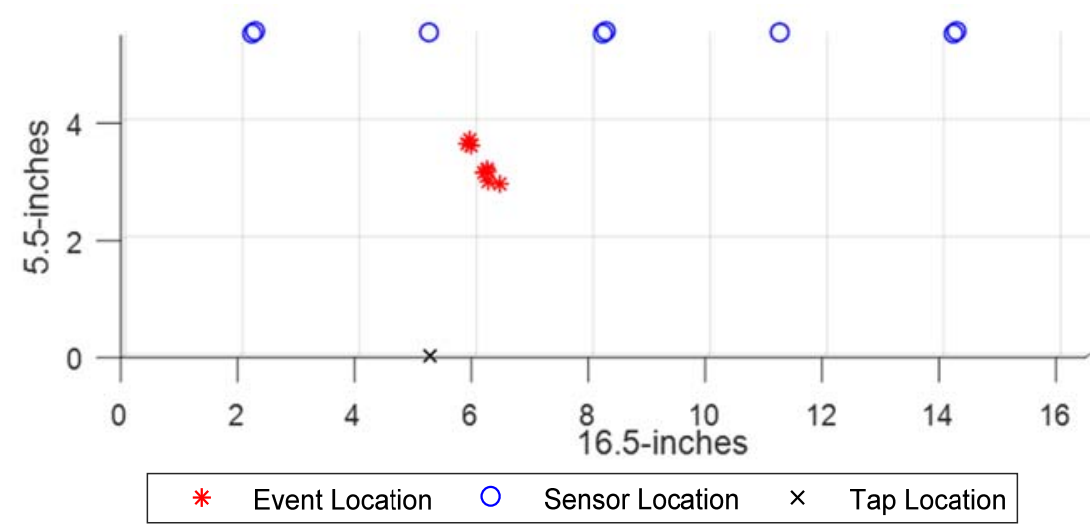

(b)

Figure 3.5. Tap testing 2-D sensor array (a) top view ( $x-y$ plane) (b) side view ( $x-z$ plane)

\subsubsection{3-D Pencil Lead Break Testing}

3-D pencil lead break tests performed on the relatively small concrete block indicated fairly accurate AE source location in the x-y plane but performed more poorly in 
distinguishing the depth of the AE source than the 2-D sensor array, as illustrated in Figure 3.6 .

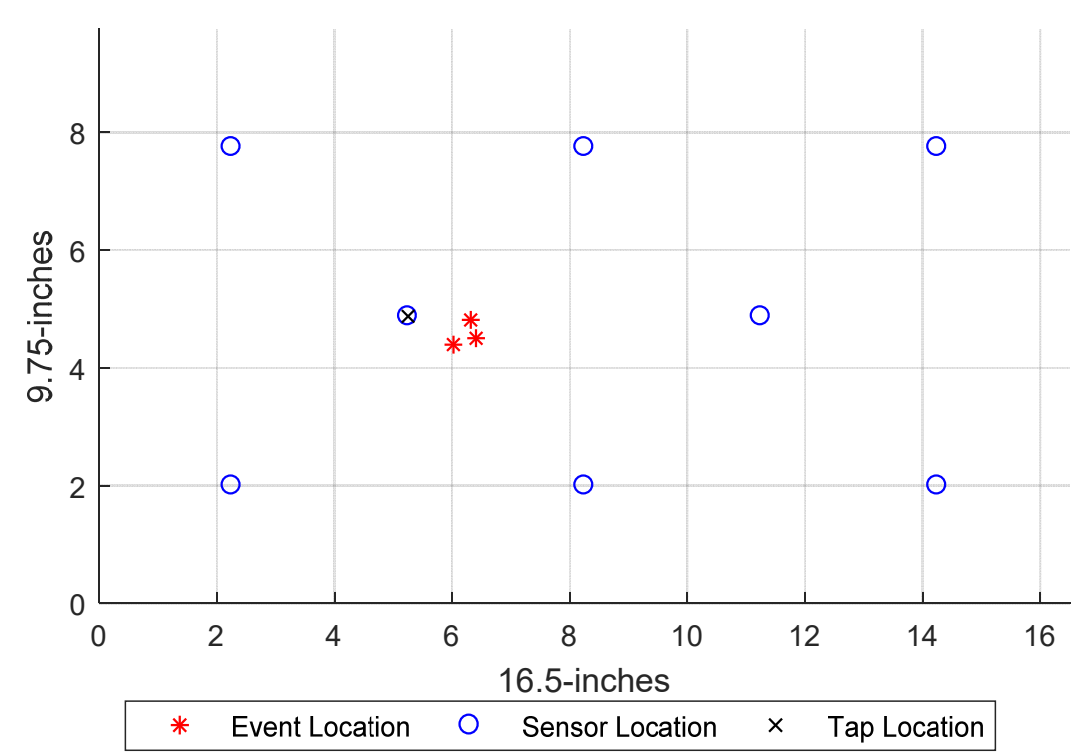

(a)

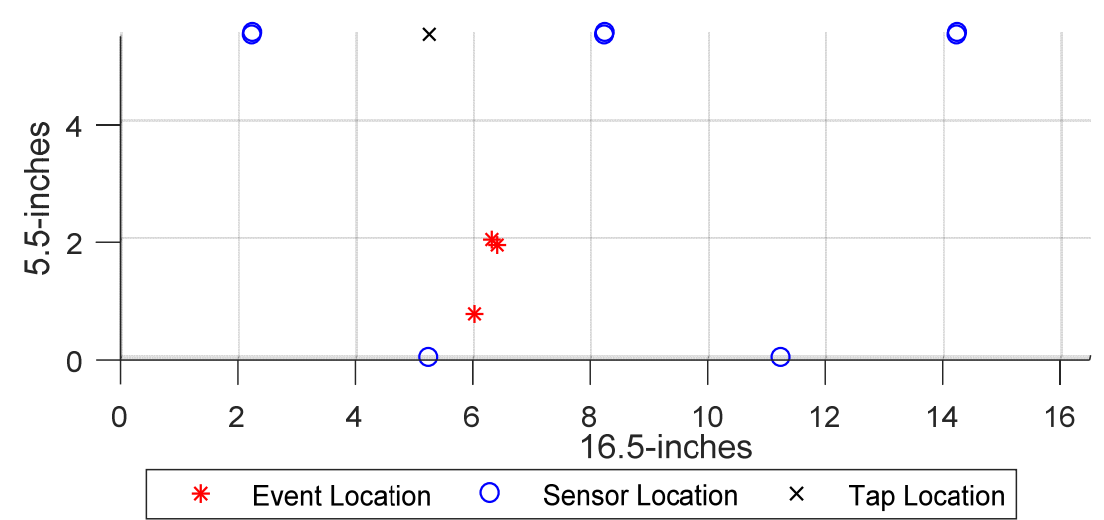

(b)

Figure 3.6. Tap testing 3-D sensor array (a) top view ( $x-y$ plane) (b) side view ( $x-z$ plane)

\subsection{AE Instrumentation Input Parameters and Data Collection}

Mistras AEwin ${ }^{\mathrm{TM}}$ Sensor Highway III monitoring system and associated AEwin ${ }^{\mathrm{TM}}$ processing software allows for user-defined inputs to refine the collected AE events. The acquisition setup parameters, as listed in Table 3.2, followed that specified by the manufacturer. Pencil lead break tests in controlled laboratory specimens confirmed the validity of these parameters (Sause, 2011). 
Table 3.2. Mistras AEwin ${ }^{\mathrm{TM}}$ Sensor Highway III Input Parameters

\begin{tabular}{|l|l|c|c|}
\hline \multicolumn{2}{|l|}{ Parameter } & Value & Units \\
\hline Threshold & 60 & decibel $(\mathrm{dB})$ \\
\hline Pre-amplifier Gain & Lower Bounds & 26 & decibel $(\mathrm{dB})$ \\
\hline \multirow{3}{*}{ Analog Filter } & Upper Bounds & 20 & kilohertz $(\mathrm{kHz})$ \\
\cline { 2 - 4 } & Lower Bounds & 400 & kilohertz $(\mathrm{kHz})$ \\
\hline \multirow{3}{*}{ Digital Filter } & Upper Bounds & 20 & kilohertz $(\mathrm{kHz})$ \\
\hline \multirow{3}{*}{ Timing Parameters } & Peak Definition Time (PDT) & 200 & microsecond $(\mu \mathrm{s})$ \\
\cline { 2 - 4 } & Hit Definition Time (HDT) & 800 & microsecond $(\mu \mathrm{s})$ \\
\cline { 2 - 4 } & Hit Lockout Time (HLT) & 1,000 & microsecond $(\mu \mathrm{s})$ \\
\cline { 2 - 4 } & Maximum Hit Duration (MDT) & 1,000 & millisecond $(\mathrm{ms})$ \\
\hline Longitudinal Wave Velocity & 13,083 & feet per second $(\mathrm{ft} / \mathrm{sec})$ \\
\hline Transverse Wave Velocity & 7,833 & feet per second $(\mathrm{ft} / \mathrm{sec})$ \\
\hline Surface Wave Velocity & 7,250 & feet per second $(\mathrm{ft} / \mathrm{sec})$ \\
\hline
\end{tabular}

If the user-defined minimum sensor hits occur within the specified time duration parameters then the $\mathrm{AEwin}{ }^{\mathrm{TM}}$ software collects fifteen features of the $\mathrm{AE}$ waveform signals including: 1) amplitude, 2) duration, 3) energy, 4) counts, 5) rise time, 6) peak frequency, 7) frequency centroid, 8) absolute energy, 9) signal strength, 10) initial frequency, 11) reverberation frequency, 12) counts-to-peak, 13) average signal level, 14) root mean square (RMS), and 15) average frequency. 


\section{CHAPTER 4 ACOUSTIC EMISSION DATA COLLECTION AND RESULTS: LABORATORY TESTING}

\subsection{Introduction}

Laboratory testing prior to and between field tests helped to establish and adapt system setup input parameters including: wave speed velocities, sensor array layouts, and amplification filters. Pull-out and three-point bending tests demonstrated the capability of AE sensing in detecting cracks. Data from three-point bending tests provided the means to associate and correlate observed cracks and stress zones to AE event locations. The pull-out and three-point bending (flexure) tests followed the work of ElBatanouny, et al. (2014), but differed with smaller scale beams and the introduction of defects to control the failure locations.

\subsection{Laboratory Specimen Preparation and Load Testing Procedures}

Laboratory pull-out and three-point bending (flexure) testing used a Tinius Olsen static tension material testing machine with a 60,000 pound-force tensile strength capacity. For pull-out tests the 5-inch x 5-inch x 12-inch (127 mm x $127 \mathrm{~mm}$ x $305 \mathrm{~mm}$ ) laboratory test beams used fast-setting QUIKRETE ${ }^{\circledR}$ and contained a single no. 4 reinforcing bar in the center of the beam, necked down off center from 0.5 -inch $(12.5 \mathrm{~mm})$ diameter to 0.25 inch $(6.25 \mathrm{~mm})$ diameter to control the point of failure. The eight sensor arrays for the test beam employed a 3-D spatial array (Figure 4.1). Silicone vacuum grease secured the sensors to the concrete beam for the relatively short duration of the laboratory tests. A load frame applied center-span force to the beams with displacement control at a rate of 0.03 in $/ \mathrm{sec}(0.762 \mathrm{~mm} / \mathrm{sec})$ until failure. 
For three-point bending tests the 5-inch x 5-inch x 24-inch (127 mm x127 mm x $610 \mathrm{~mm}$ ) laboratory test beams used fast-setting QUIKRETE ${ }^{\circledR}$ and contained two no. 4 reinforcing bars spaced roughly 1.5 -inches $(38 \mathrm{~mm})$ from the bottom and adjacent side and were necked down in the center from 0.5 -inch $(12.5 \mathrm{~mm})$ diameter to 0.25 -inch $(6.25 \mathrm{~mm})$ diameter to control the point of failure. The eight sensor arrays for the test beams were either a 2-D planar array (Figure 4.2) or 3-D spatial array (Figure 4.3). Silicone vacuum grease secured the sensors to the concrete beam for the relatively short duration of the laboratory tests. A load frame applied center-span force to the beams with displacement control at a rate of $0.03 \mathrm{in} / \mathrm{sec}(0.762 \mathrm{~mm} / \mathrm{sec})$ until failure.

\subsubsection{Pull-out Test Data Collection}

The pull-out test recorded ninety AE events. The concentration of AE events was around the observed cracking as seen in Figure 4.1. Fourtyeight of the 90 events led to anomalous location estimates with the estimated location appearing underneath one of the eight sensors. Subsequent laboratory testing attempted a different sensor connection such as removing the metal boxes and use of different couplants (i.e. epoxy vs. silicone vacuum grease). The recorded AE events, excluding the on-sensor events, generally concentrated around the observed cracking. As mentioned earlier, the AE sensor connections to the beam were secured into prefabricated metal armored boxes made from hollow square stock and then attached to the beam specimen with 5-minute epoxy. The metal boxes cut from hollow stock may not fit truly flush to the sensor which would allow for a possible air gap between the sensor wear plate and steel box. This air gap could create some disturbance or throw errors in the source location that could result in the AE event being located directly

at the sensor. However, the pull-out tests described here confirmed that after eliminating 
the on-sensor $\mathrm{AE}$ events, the remaining $\mathrm{AE}$ events had a relatively strong correlation to observed cracking. It should be noted that the investigators later learned that the underlying triangulation algorithm is prone to this anomaly when using rectangular arrays of sensors. A staggered triangular grid geometry tends to avoid these anomalies.

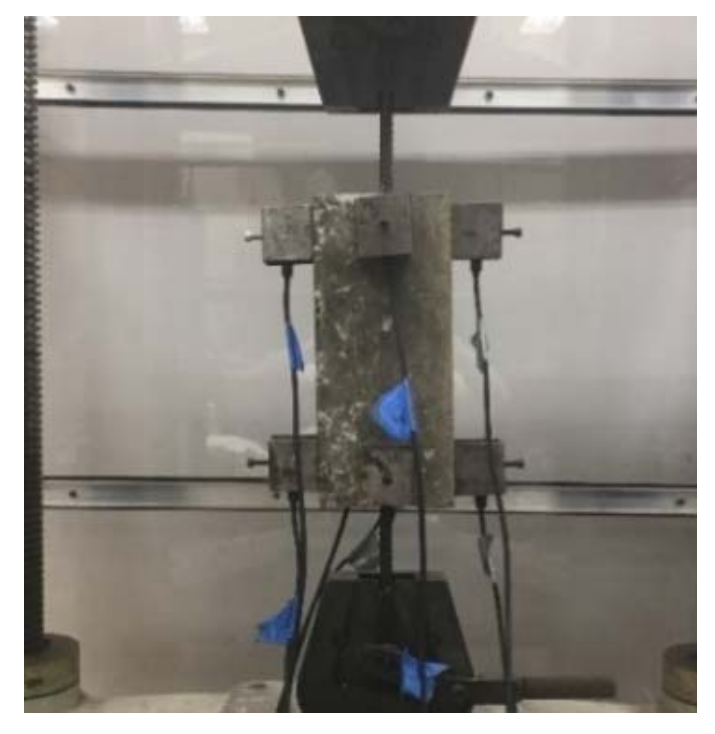

(a)

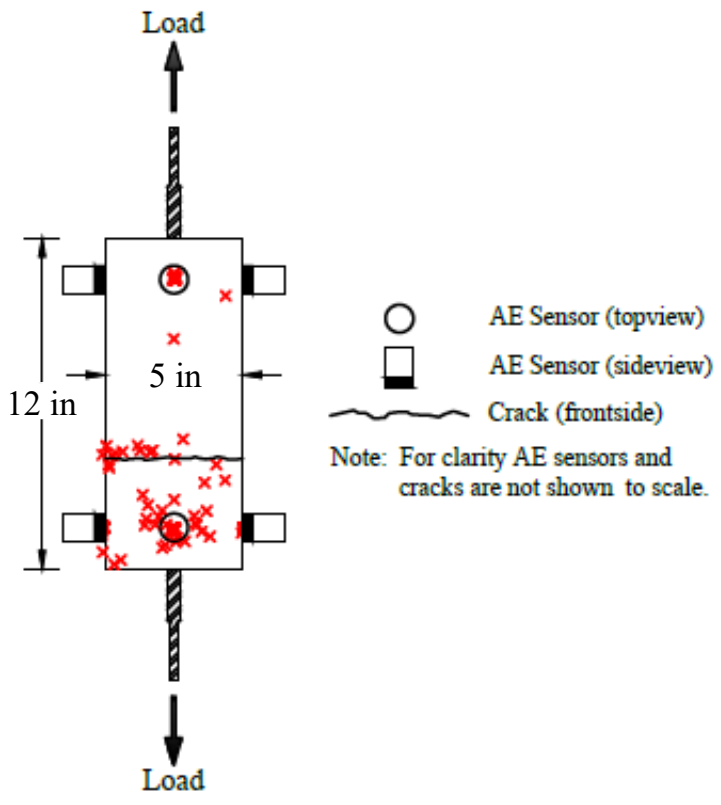

(b)

Figure 4.1. Laboratory pull-out test of reinforced concrete beam with 3-D sensor array. (a) Photograph of 3-D sensor array prior to testing, (b) Schematic of 3-D sensor array including AE event locations, sensors, and observed cracks

\subsubsection{Three-Point Bending Data Collection}

This section describes data collected from relatively small laboratory reinforced concrete beam specimens under three-point bending to evaluate the performance of $\mathrm{AE}$ sensing in detecting cracks. The bending tests used both 2-D and 3-D sensor array configurations. The 2-D array placed eight acoustical emission sensors on one side of the beam (as seen in Figure 4.2a). The 3-D array placed eight acoustical emission sensors on the beam with three sensors on each side and two sensors on the top as seen in Figure 4.3a. High vacuum grease secured the sensors to the beam and acted as a couplant for elastic 
waves. This connection procedure eliminated the metal sensor armored boxes in order to determine if on-sensor AE events were caused by poor coupling between the sensor and source material.

\subsubsection{2-D Sensor Array}

The laboratory three-point bending test employing the 2-D planar sensor array recorded a total of $142 \mathrm{AE}$ events when loaded to failure (Figure 4.2). Observed cracking took place in the middle of the beam, nearly in line with the point of loading. The recorded AE event locations were generally within 2 inches $(50 \mathrm{~mm})$ from the observed cracks. Recorded AE events with locations outside of this region may be due to other effects and events. The possibilities include internal cracking, erroneous AE events location estimates that typically occur directly under a sensor location, and reflection and guiding of the elastic waves by the crack surfaces.

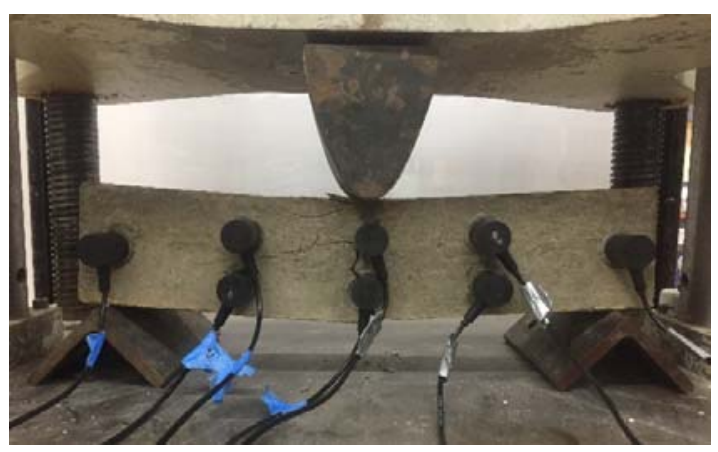

(a)

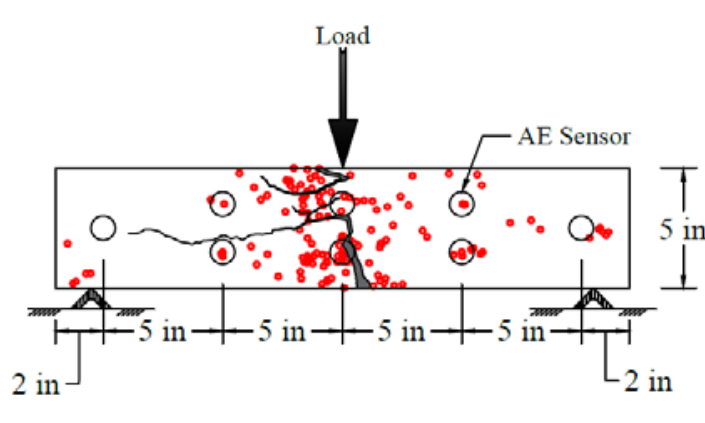

(b)

Figure 4.2. Laboratory three-point bending test of reinforced concrete beam with 2-D sensor array. (a) Photograph of 2-D sensor array at the completion of testing, (b) Schematic of 2-D sensor array including AE event locations, sensors, and observed cracks

Categorization of the observed $\mathrm{AE}$ events based on location produced three groupings; 1) AE event on or near an observed crack, 2) AE event not on or near an observed crack, and 3) AE event directly at an AE sensor location. Analyzing the 15 
waveform features collected from each AE signal, by the AEwin ${ }^{\mathrm{TM}}$ Sensor Highway III, led to a comparison of the three groups. Although a clear determination of the AE event features from the observed cracks was not immediately identified, it is of note the linear distribution when plotting AE event amplitudes with respect to AE event durations, Figure 4.3.

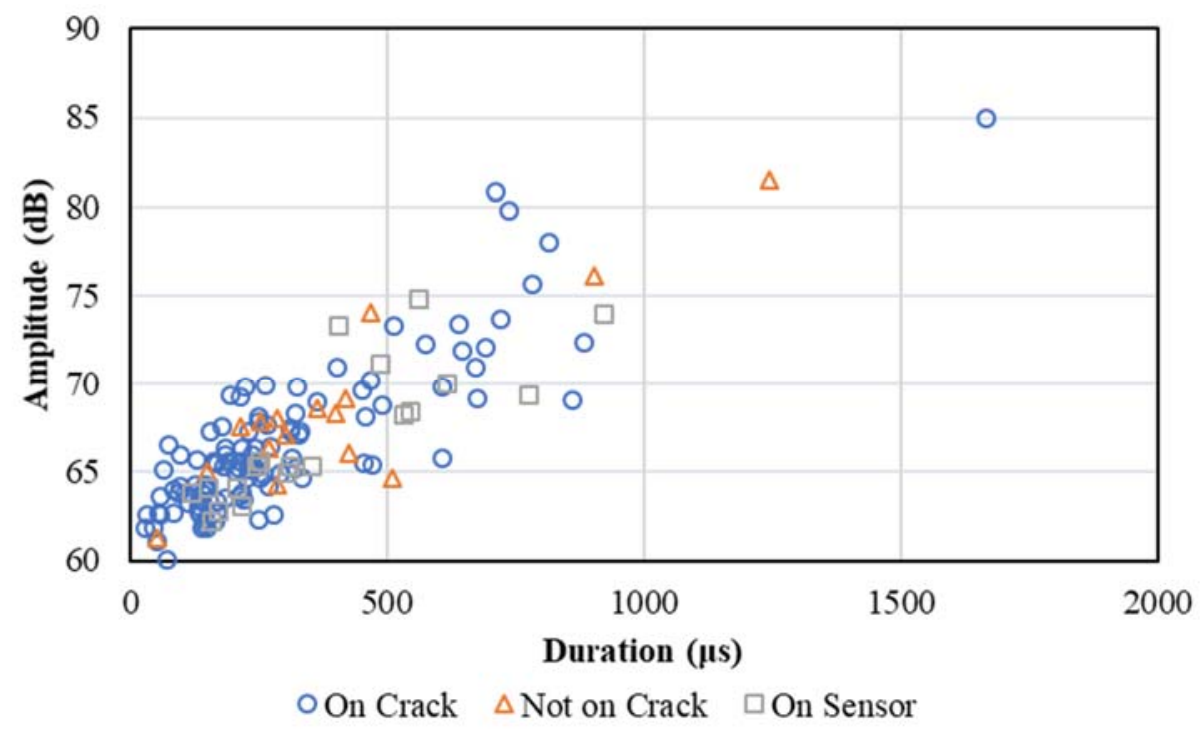

Figure 4.3. AE event duration vs. amplitude categorized by proximity to observed cracks

\subsubsection{3-D Sensor Array}

The laboratory three-point bending test employing the 3-D sensor array recorded a total of $111 \mathrm{AE}$ events when loaded to failure, Figure 4.4. The observed cracking took place on the left side of the beam, largely as shear cracking. No cracks were observed on the right side of the test beam. The locations of the recorded $\mathrm{AE}$ events were almost entirely on the left side of the beam, where all the cracks occurred. 


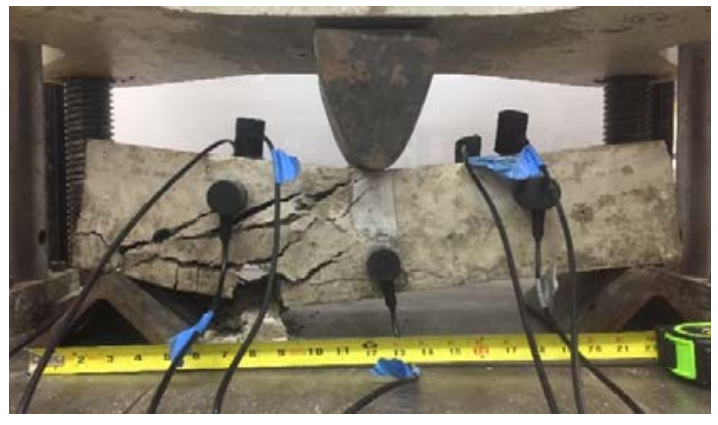

(a)

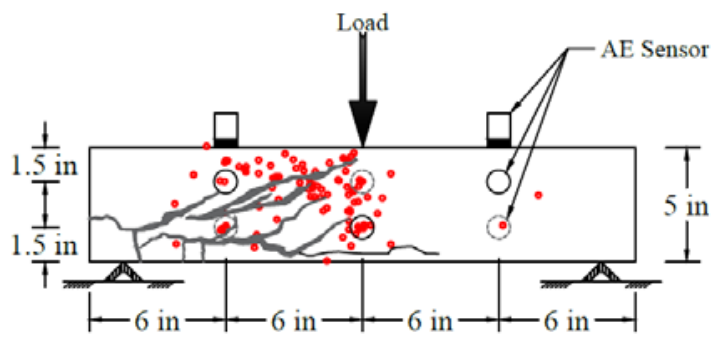

(b)

Figure 4.4. Laboratory three-point bending 3-D sensor array. (a) Photograph of 3-D sensor array at the completion of testing, (b) Schematic of 3-D sensor array including AE event locations, sensors, and observed cracks

These tests indicated a strong correlation between observed cracks and recorded AE event locations. The 2-D planar sensor array configuration showed greater accuracy in locating surface cracks. A possible reason for the discrepancy between the 2-D and 3D sensor arrays stems from the differences in the AE event source-locating algorithms. The 2-D source-locating algorithm relies on surface wave speed velocities that were determined from pencil lead break tests. The 3-D source locating algorithm utilizes longitudinal, transverse, and surface wave velocities of which the longitudinal and transverse wave velocities were estimated from published correlations between these three wave velocities in standard concrete and are at best an approximate representation of the beam tested (Lee et al., 2016).

\subsection{Conclusions from the Results of Laboratory Testing}

Laboratory experiments on relatively small reinforced concrete beam specimens under pull-out and three-point bending yielded proof of concept and guidance on data acquisition setup parameters for field testing. These include:

1. Appropriate amplitude and duration filters to remove background noise; 
2. Verification of wave mode velocities through pencil lead break tests;

3. Verification of correlation between AE events and observed cracks; and

4. Selection of appropriate sensor array.

Laboratory pull-out and three-point bending (flexure) testing yielded correlations between $\mathrm{AE}$ events and cracking and indicated that a 2-D planar sensor array was more accurate in $\mathrm{AE}$ event source location than a 3-D sensor array. One possibility for the difference in accuracy is the use of measured wave speed velocity used in the 2-D location algorithm versus the empirical relationship estimates of wave speed velocities used in the 3-D sensor array. 


\section{CHAPTER 5 ACOUSTIC EMISSION DATA COLLECTION AND RESULTS: FABRICATION}

\subsection{Introduction}

This chapter focuses on the use of Acoustic Emission (AE) technology as a potential Quality Assurance/Quality Control (QA/QC) procedure in source locating of AE events associated with cracking of prefabricated and pre-stressed reinforced concrete NEXT beams and NEBT girders during specific prefabrication processes including; detensioning and craned lifting from form beds. NEXT beams and NEBT girders are of interest to this study and were chosen for testing based on their regular use as PBES in the northeastern United States where these elements and any cracking are subject to harsh and fluctuating weather along with the application of de-icing materials that can cause accelerated corrosion and degradation of concrete.

\subsection{NEXT Beam Testing}

The testing of full-sized pre-stressed and prefabricated concrete girders at the manufacturing site, fabricated at J.P. Carrara and Sons, Inc. in Middlebury, VT; began with observations of detensioning and crane movement operations. The observations indicated that it would be advantageous to have an untethered instrument package. This prompted modifications to the instrumentation to include a portable power supply and wireless remote network connection, which allowed for untethered operations. The next step was to record AE events of a NEXT beam during the detensioning processes and craned removal of the beam from the formwork deck and placement on wood blocks for additional finishing steps, curing and storage. The sensing configuration arranged eight AE sensors in a 2-D array on the top deck of the NEXT beam off-center toward one end, which had a 
skew of approximately 14 degrees. The intent of the sensor layout was to capture the acoustic emission events that may result from the release of energy and load transfer during the torch cutting of the steel tensioning strands within the NEXT beam. Steel armor boxes and 5-minute epoxy attached the sensors to the top of the beam. The formwork geometry prevented attaching the sensors to the side of the beam and using 3-D estimates of event locations. Instead, the estimation of event locations used a 2-D plate model of the beam. The instrumentation recorded $38 \mathrm{AE}$ events during the detensioning process as illustrated in Figure 5.1. As a standard practice of the fabricators, the torch cutting of prestressing strands was synchronized to cut wires from the same strand simultaneously on each side of the NEXT beam in a gradual process that sequentially releases the entire tension within that strand and transferring the load into the beam. The loading exerted on the beam by strand cutting was less dynamic than originally anticipated, primarily due to the slow cutting of individual wire within the strands releasing only a relatively small amount of energy per cut. The strand cut sequence followed a pattern that alternated sides of the beam cross section in an effort to balance the load transfers. The recorded AE events tended to occur closer to the end of the NEXT beam with multiple events stacked onto one another occurring along the underlying integral girder containing the tensioning strands. 


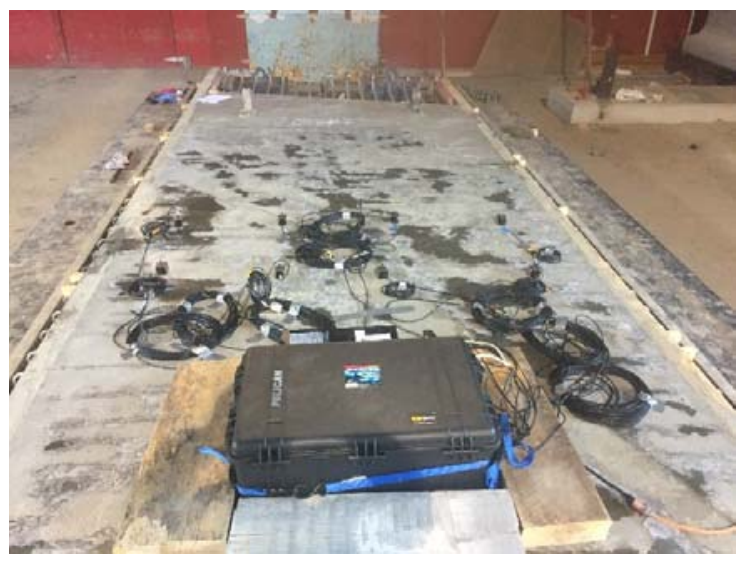

(a)
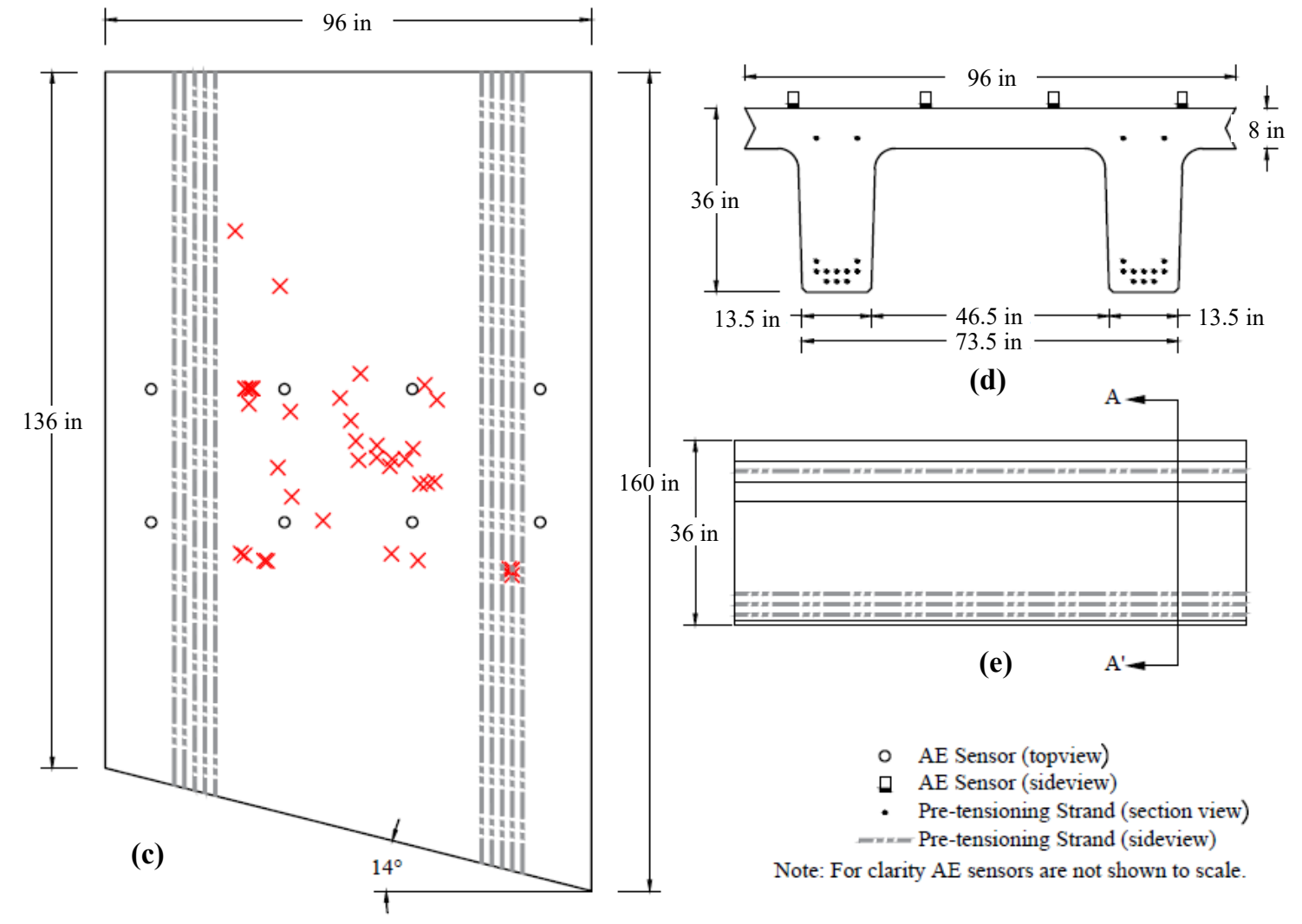

(d)

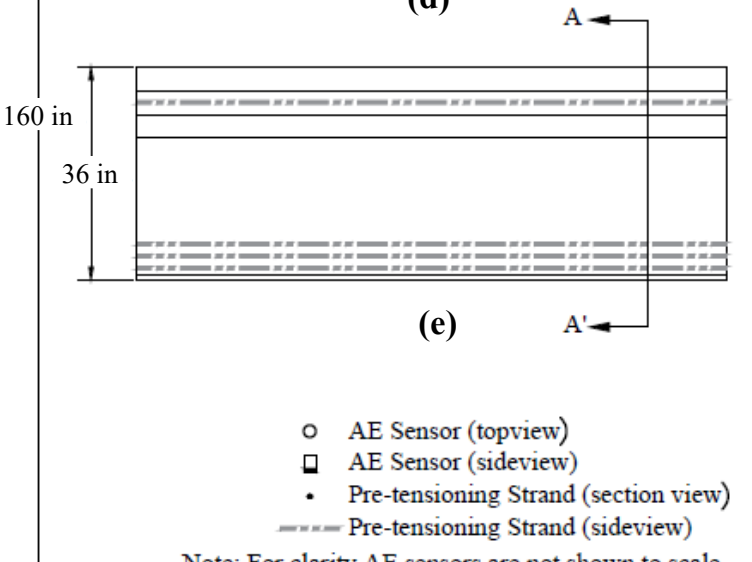

Note: For clarity AE sensors are not shown to scale.

Figure 5.1. NEXT beam field testing of detensioning with 38 recorded events (a) field testing equipment setup, (b) torch cutting of pre-tensioning strands, (c) plan view of AE events on NEXT beam, (d) section view of NEXT beam, and (e) profile view of NEXT beam

Twenty AE events were recorded during the craned removal of the NEXT beam from the form bed, Figure 5.2. Upon completion of detensioning, overhead tracked gantry cranes lifted the beams from the form decks. The lifting and movement processes stress 
the beam as it breaks loose from the form deck and changes the vertical support to only four load points, two pairs of points at approximately quarter spans. Immediately after hoisting out of the form deck, the beam appeared to camber from an initially flat profile over roughly one minute, presumably due to tension stresses exerted by the embedded steel in the lower sections of the girder and the release of the geometric constraints imposed by the form deck. The cambering process along with change of gravity load paths during hoisting has the potential for creating areas of concentrated stress/strain. This is a period of interest for AE event monitoring, as cracking may occur.

$\mathrm{AE}$ event data collection during the detensioning and craned form removal of the NEXT beam were the first field data collection performed with the equipment and corresponding software. The results were 1) The instrumentation successfully recorded $\mathrm{AE}$ events, 2) event locations loosely correlated with the integral girders during the detensioning process where the pre-tensioning strands were being cut, and 3) the equipment alterations for wireless connectivity and portable power supply were successful. The collected AE data show that many AE events occurred during the craned removal of the NEXT beam from the formwork deck. Upon lifting, observation of the beam found it to slowly camber a few inches over roughly a minute. The strain on the concrete due to this camber may be responsible for more AE events and potential internal cracking than the dynamics and load redistribution of detensioning; had the sensor array been focused on the middle portion of the NEXT beam. 


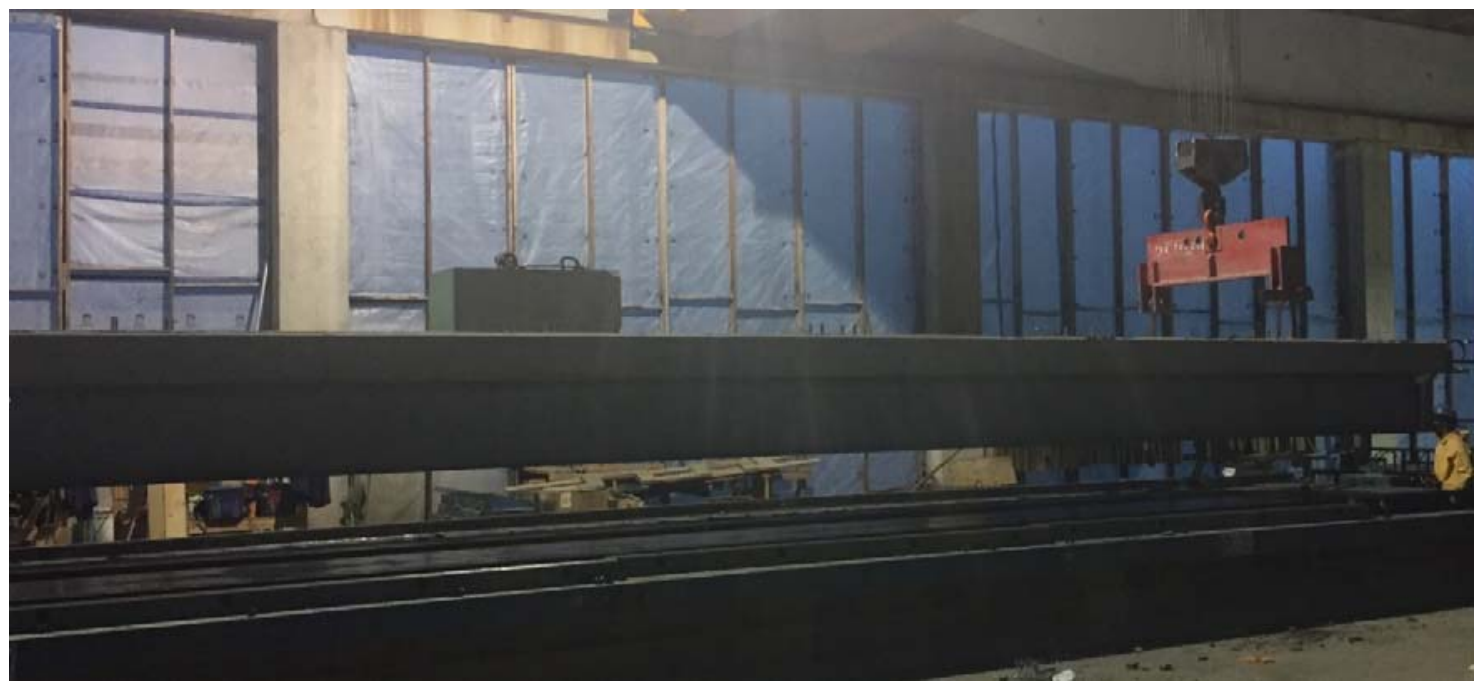

(a)
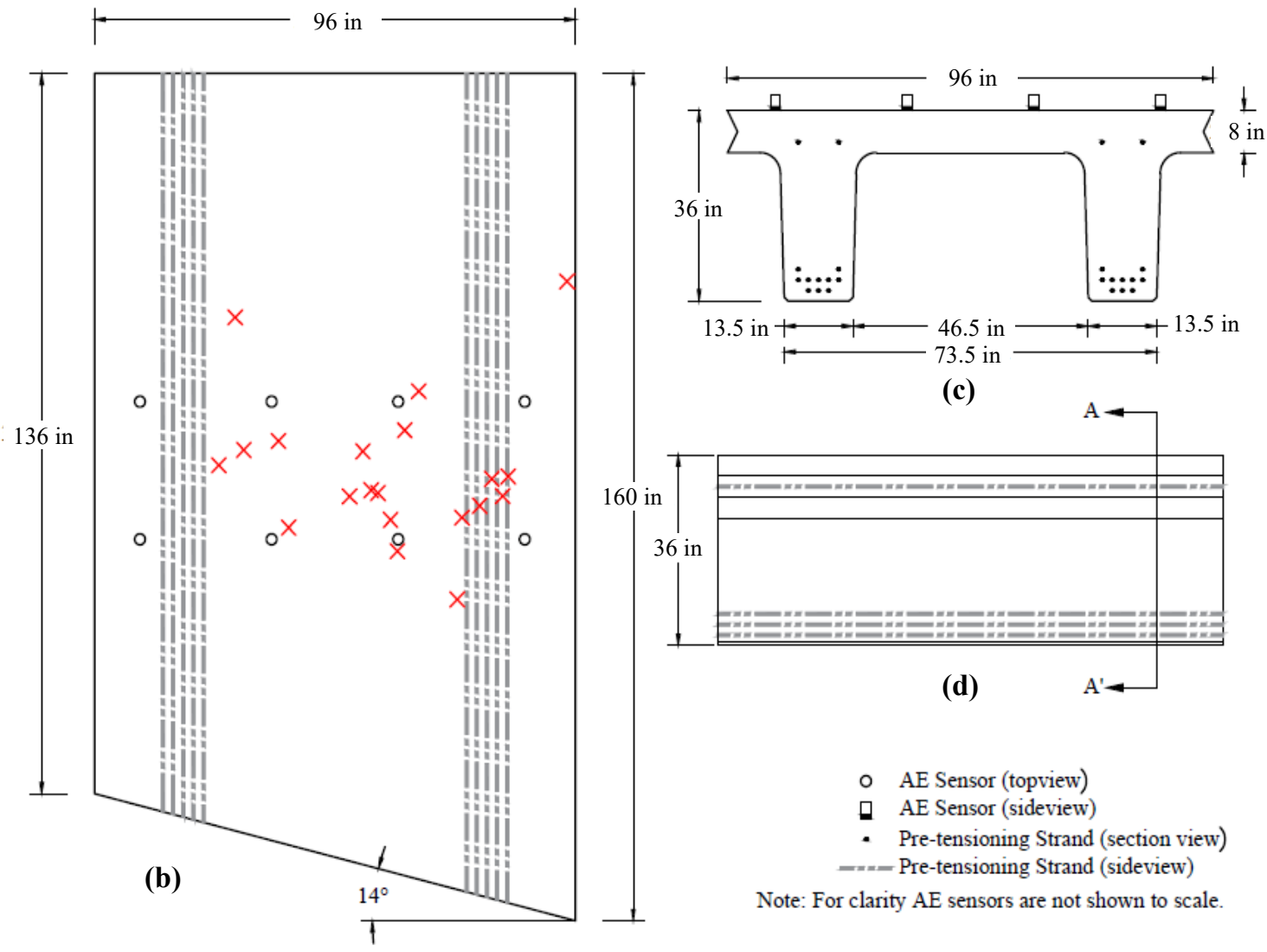

(c)

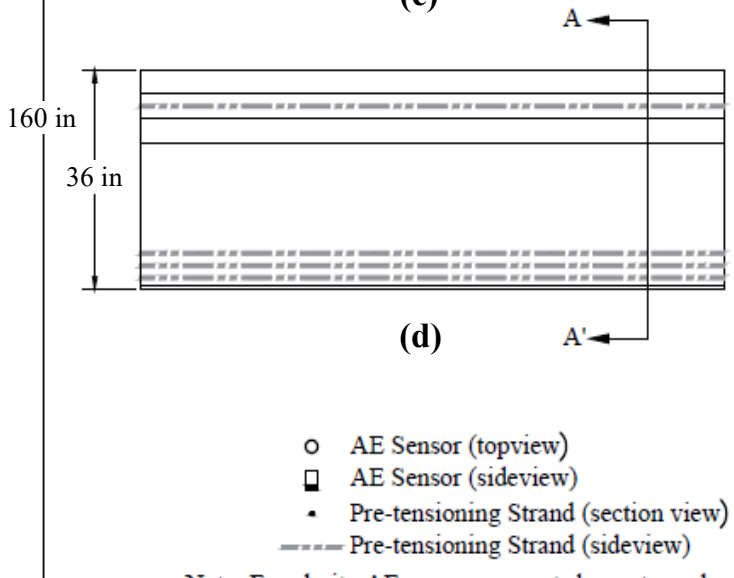

Note: For clarity AE sensors are not shown to scale.

Figure 5.2. NEXT beam field testing of craned form removal with 20 recorded events (a) crane lifting, (b) plan view of AE events on NEXT beam, (c) section view of NEXT beam, and (d) profile view of NEXT beam 


\subsection{NEBT Girder Testing}

Two additional fabrication facility field site investigations, at J.P. Carrara and Sons, Inc. in Middlebury, VT; recorded AE events from the web of a NEBT girder during the detensioning processes, craned form removal, and end bulkhead removal.

\subsubsection{NEBT 3-D Sensor Array Data Collection}

The first sensor configuration placed eight sensors in a 3-D array, four sensors on each side of the web of the NEBT, within the end zone transfer depth, estimated as 60 times the diameter of the pre-tensioning strands or 36-inches $(914 \mathrm{~mm})$ per 5.11.4.1 of AASHTO Bridge Design Specifications (PCINE-14-ABC, 2014). The intent of the sensor array placement was to focus $\mathrm{AE}$ data collection in an area predicted to have the greatest stress/strain transfer during loading from the release of the torch cut pre-tensioning strands. Metal armor boxes and 5-minute epoxy secured the sensors to the NEBT girder. The data acquisition unit operated in an untethered configuration. Estimation of AE event locations used a simplified model that included only the web of the NEBT girder. The end zone of the NEBT girder had three empty post tensioning tubes running roughly in the middle of the girder that were each approximately 4-inches $(100 \mathrm{~mm})$ in diameter. End zone reinforcement pattern details include; six no. 5 rebar in the top flange running the length of the beam, fourteen no. 5 rebar in the web running the length of the beam, and four no. 6 and six no. 4 rebar in the bottom flange running the length of the beam. The end zones also had four stirrups in the end 11-inches $(275 \mathrm{~mm})$ of the NEBT along with an additional seven no. 6 rebar spaced at 3 -inches $(75 \mathrm{~mm}$ ) running perpendicular to the NEBT in both the top and bottom flanges behind the stirrups. 
The detensioning process produced 466 recorded AE events, Figure 5.3. The detensioning process was similar to that of the NEXT beam with synchronized single wire cutting of strands on both ends of the girder. The recorded AE events tended to occur within the end zone transfer depth. The AE event occurrence locations were dense in the area of the pre-tensioning strands and web and less dense in areas furthest from the pretensioning strands. There were also noticeable conglomerates of events located directly at the sensor locations on the surface of the NEBT. Visual observations detected and documented end zone cracking immediately after the completion of the detensioning process, Figure 5.3. End zone cracks ranged in length from 5-inches to 20 -inches $(127 \mathrm{~mm}$ to $508 \mathrm{~mm}$ ). 


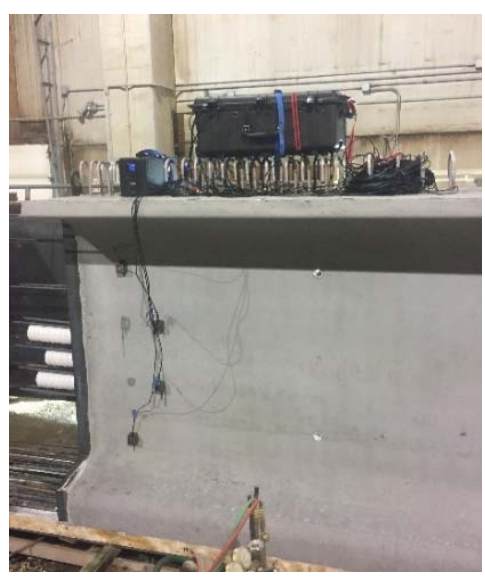

(a)

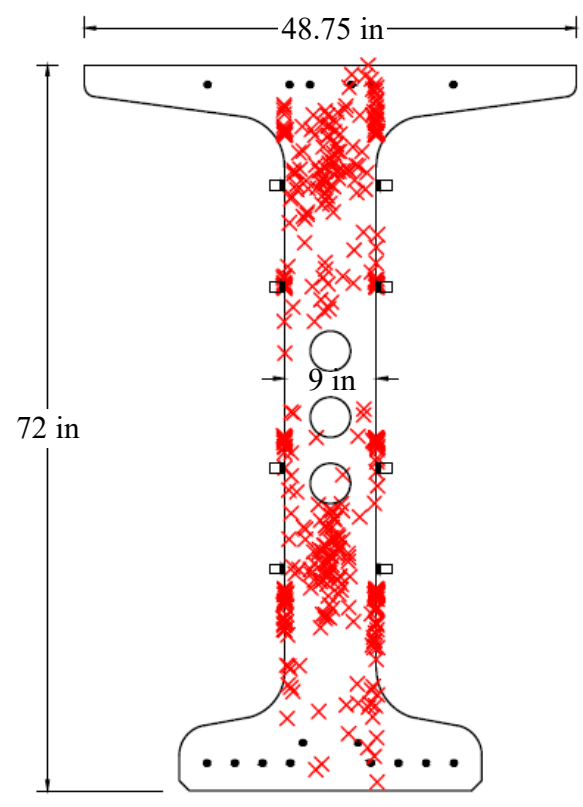

(c)

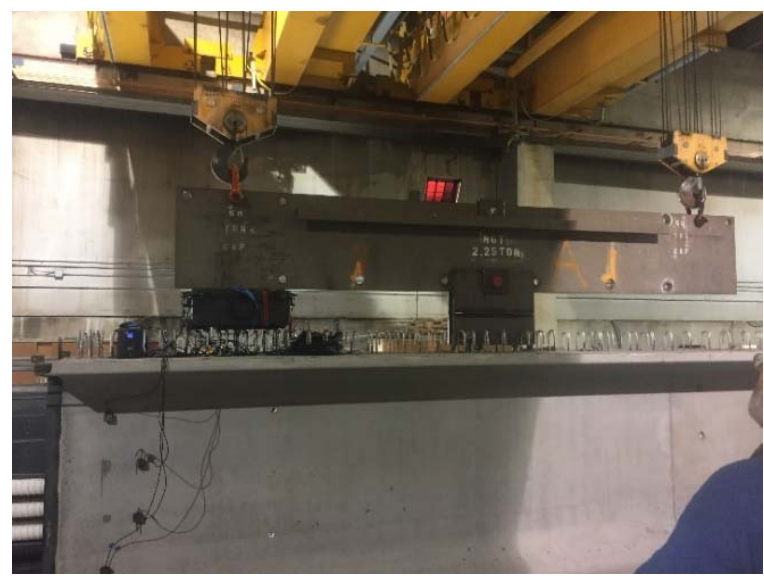

(b)

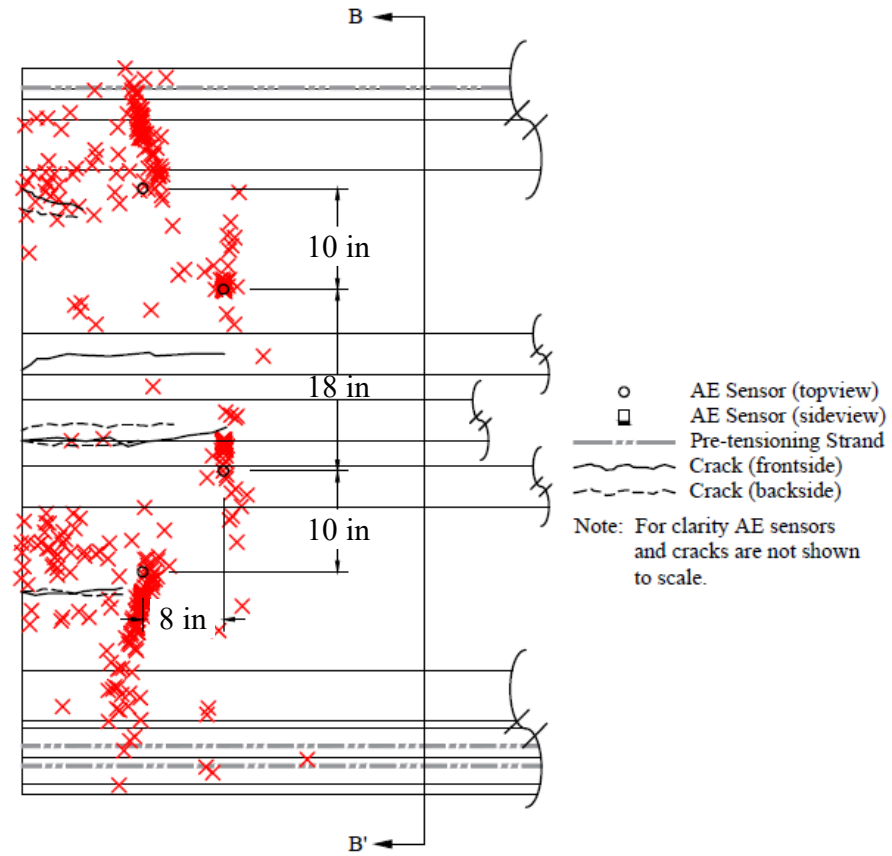

(d)

Figure 5.3. NEBT girder 3-D sensor array field testing of detensioning with 466 recorded events (a) AE equipment setup, (b) gantry crane connection to NEBT, (c) section view of NEBT beam, and (d) profile view of NEBT beam

Recorded amplitude distributions of detensioning with a 3-D sensor array between the threshold lower limit $(60 \mathrm{~dB})$ and upper limit $(99 \mathrm{~dB})$ indicate large concentrations of $\mathrm{AE}$ events at the lower and upper amplitude limits with a near uniform distribution of $\mathrm{AE}$ events between these limits (Figure 5.4). This may indicate a revision is required to the 
amplitude thresholds; however, subsequent testing using a 2-D sensor array indicate this range does capture the most relevant $\mathrm{AE}$ events.

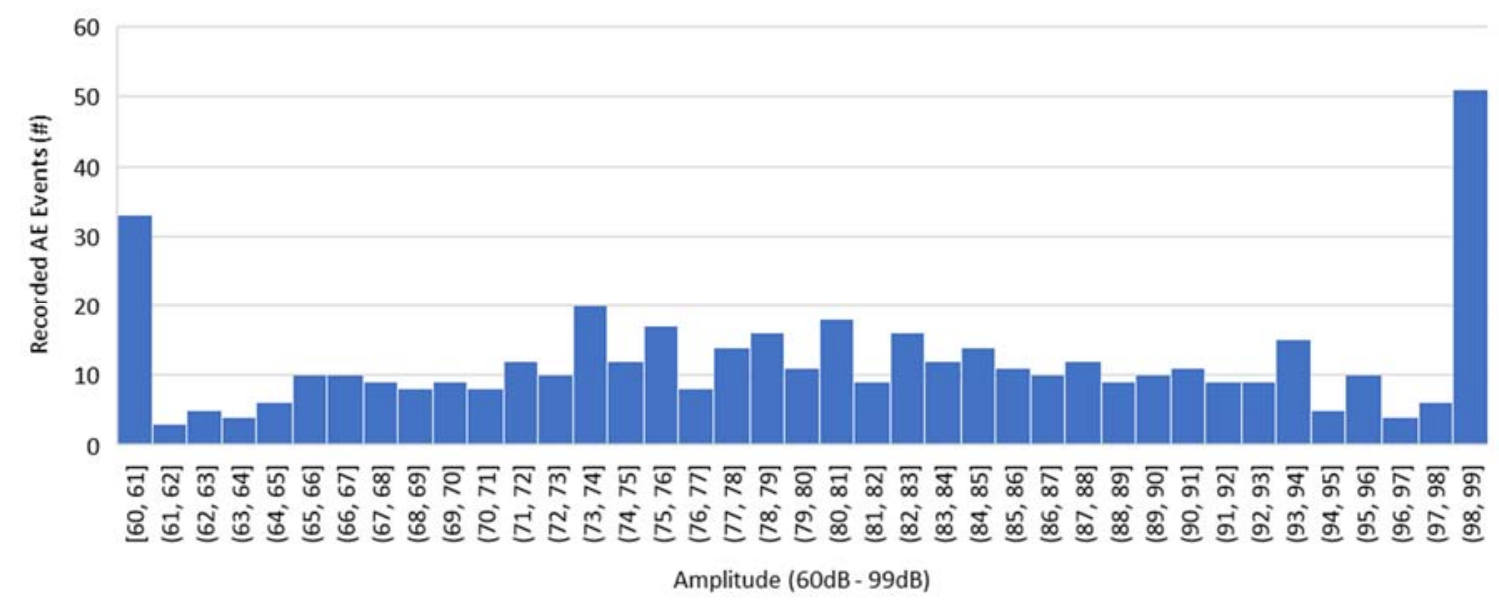

Figure 5.4. Amplitude distribution for NEBT beam 3D sensor array field testing of detensioning with 466 recorded events

There were a total of 22 pre-tensioning strands that were torch cut during the AE data collection using a 3-D sensor array. The AE data collected during detensioning were analyzed by plotting the number of AE events recorded over time and indicates 22 separate spikes in AE activity that correspond to a respective torch cutting event of a pre-tensioning strand as shown in Figure 5.5. 


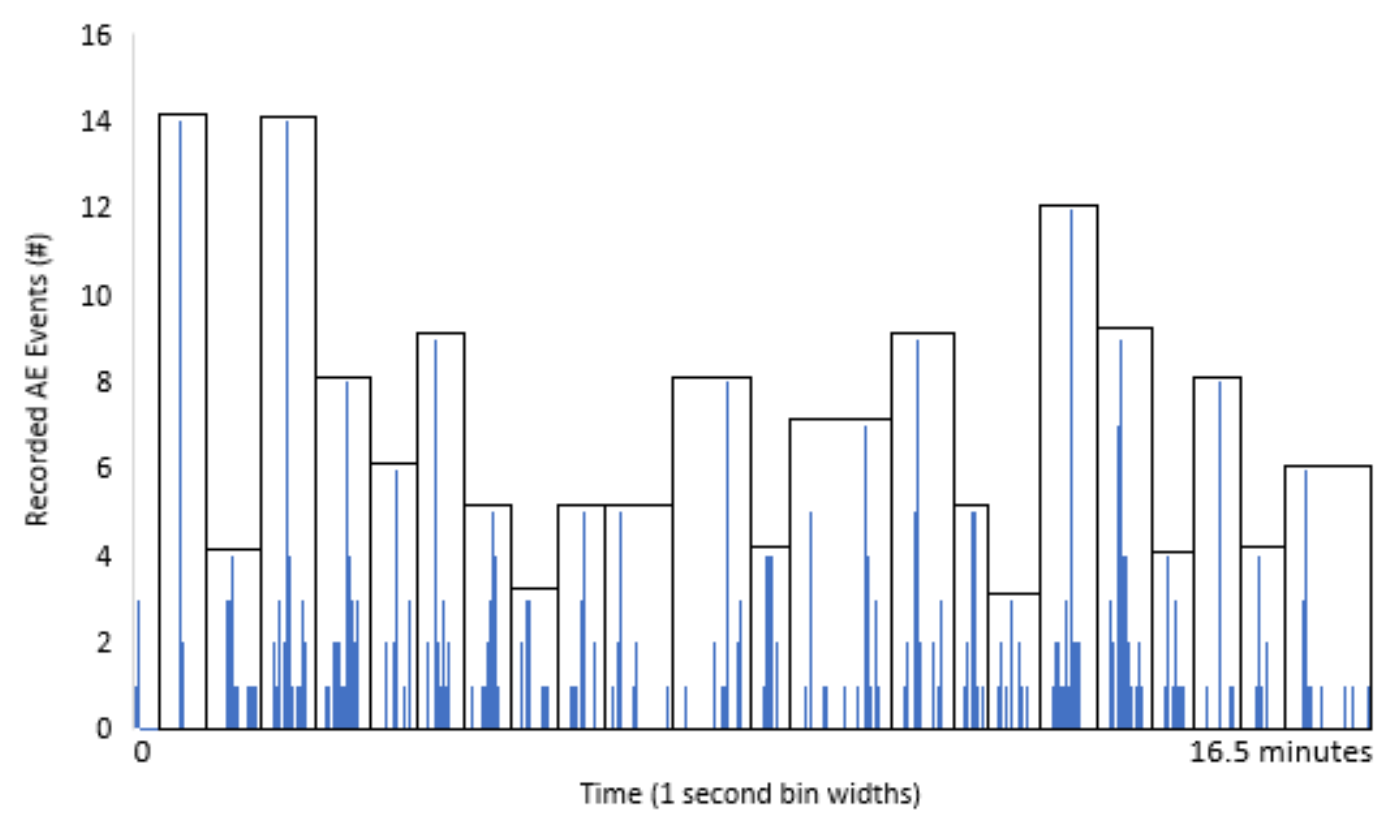

Figure 5.5. NEBT beam 3D sensor array field testing of detensioning with 466 recorded events over time with delineation of recorded $\mathrm{AE}$ events to each torch cutting of a pre-tensioning strand

As described in Chapter 2, the b-value analysis is a statistical regression of the $\mathrm{AE}$ event peak amplitudes that requires a complete loading-unloading cycle for calculation. An abrupt decrease of the calculated b-value indicates the occurrence of damage. Although the loading of the NEBT girders during detensioning is irregular by the varying loading location, the b-value analysis can be applied by analyzing the recorded AE events associated with each torch cutting of a respective pre-tensioning strand. Applying the bvalue analysis, low b-values could indicate the occurrence of damage.

The b-value regression plots by pre-tensioning strand are shown in Figure 5.6. A statistical break point analysis was used to break the curves into segmented linear regressions. Based on the shape of the curves the middle regions appear to be most representative of the data and were used in developing the b-value statistic. 

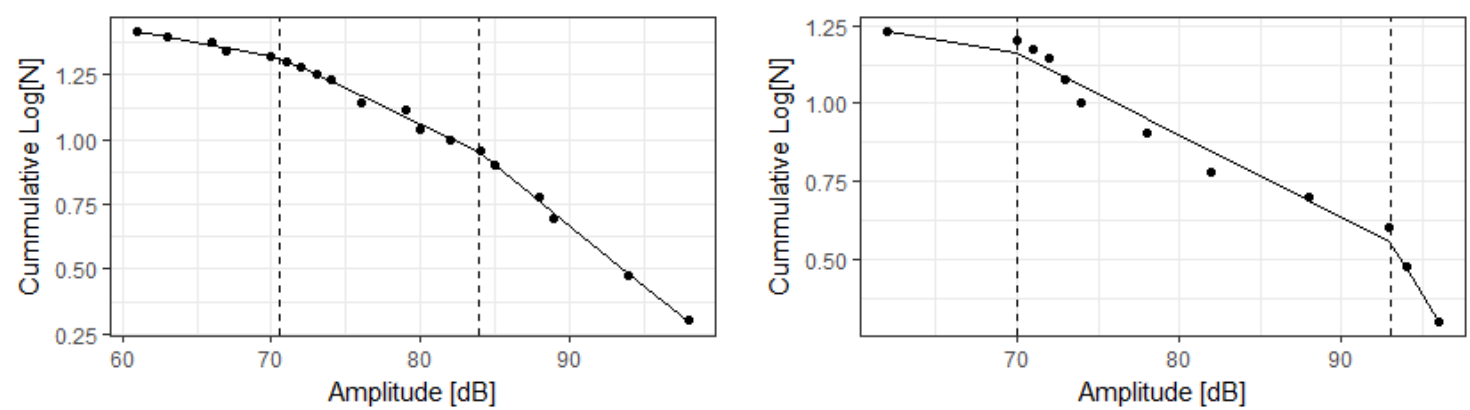

Strand 1

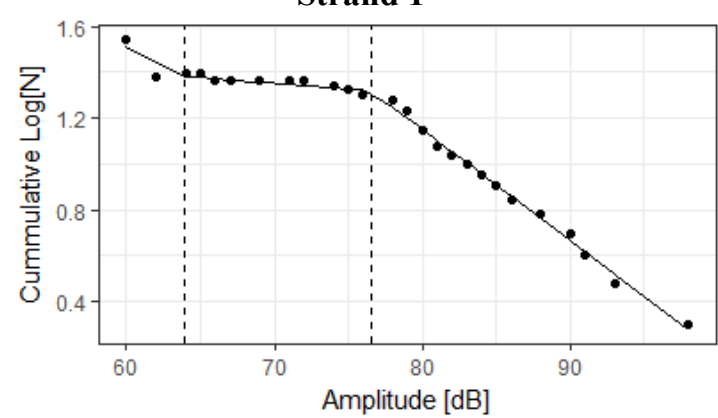

Strand 2

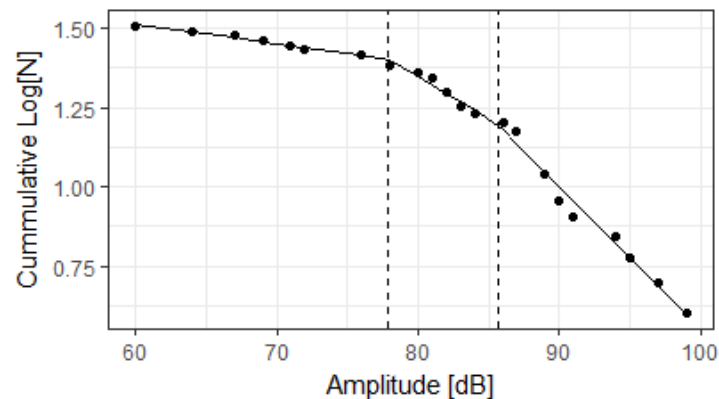

Strand 3

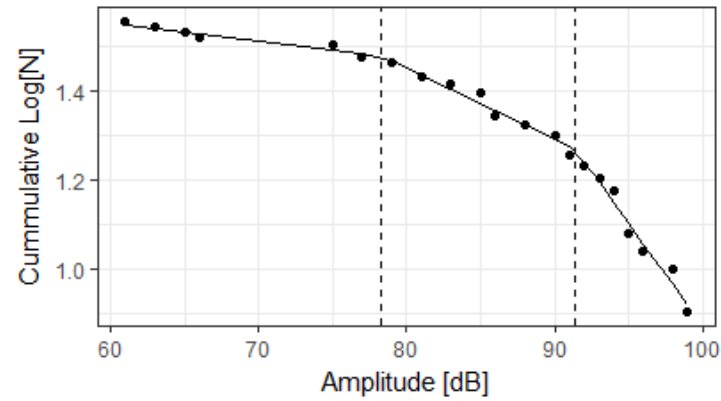

Strand 4

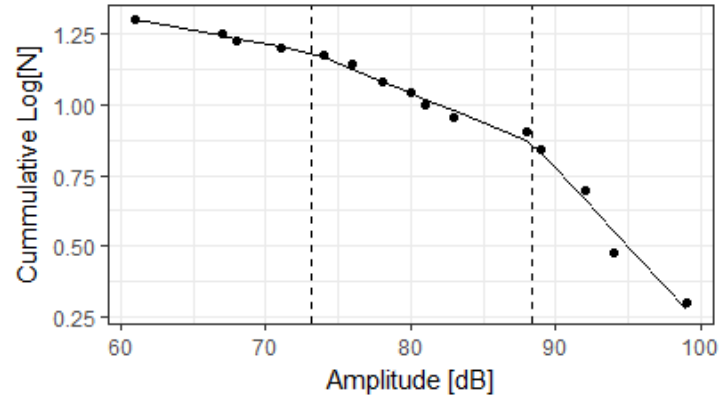

Strand 5

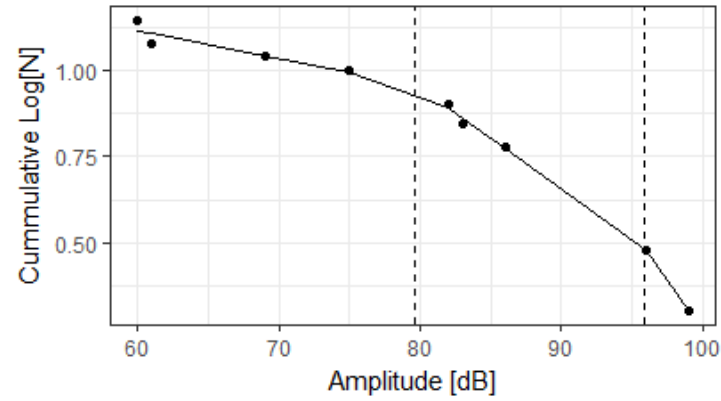

Strand 7

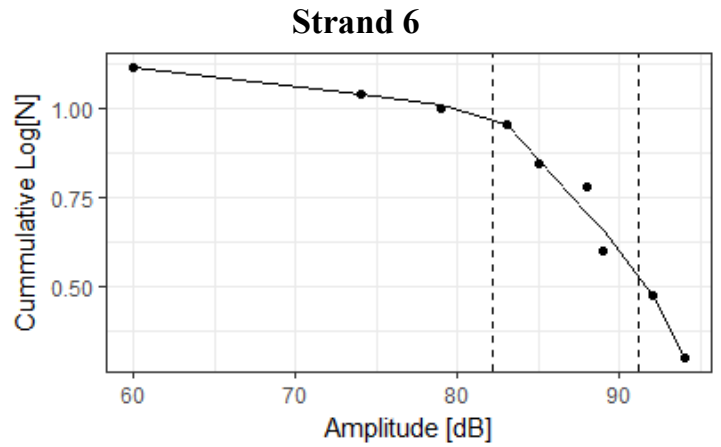

Strand 8 

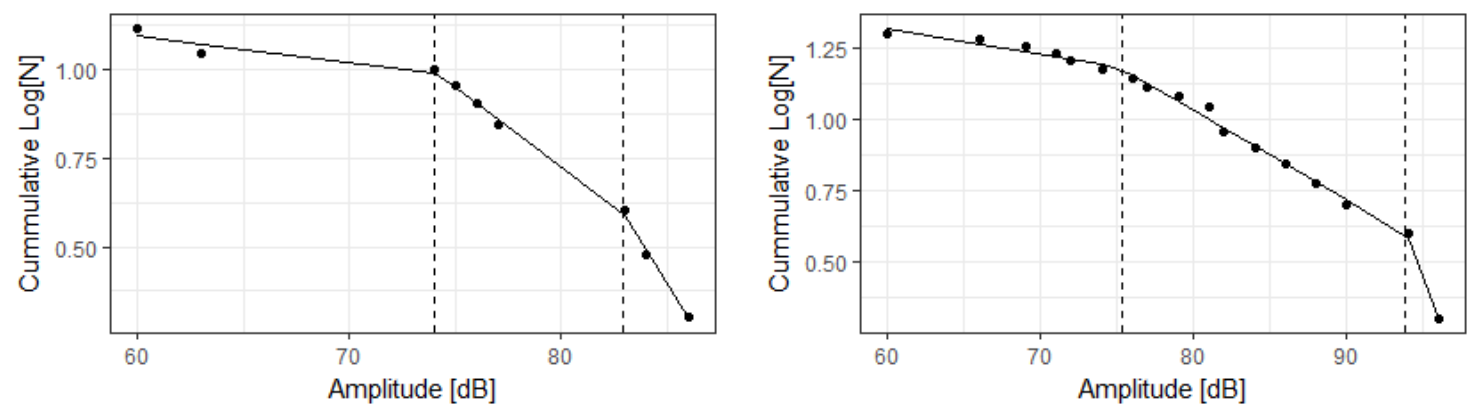

Strand 9

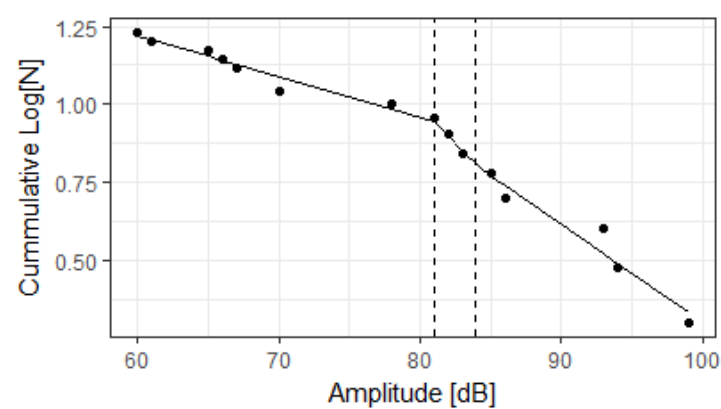

Strand 10

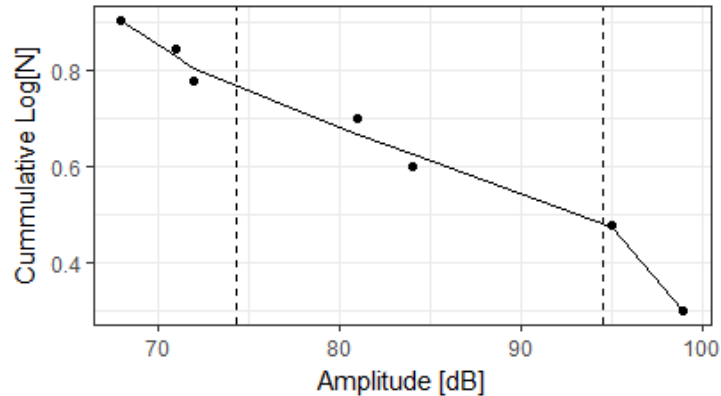

Strand 11

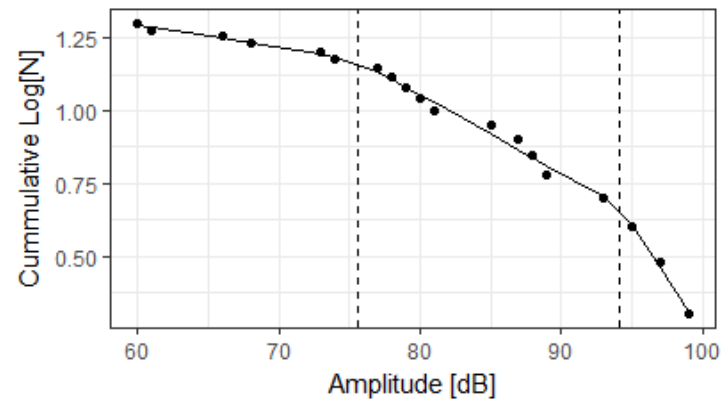

Strand 13

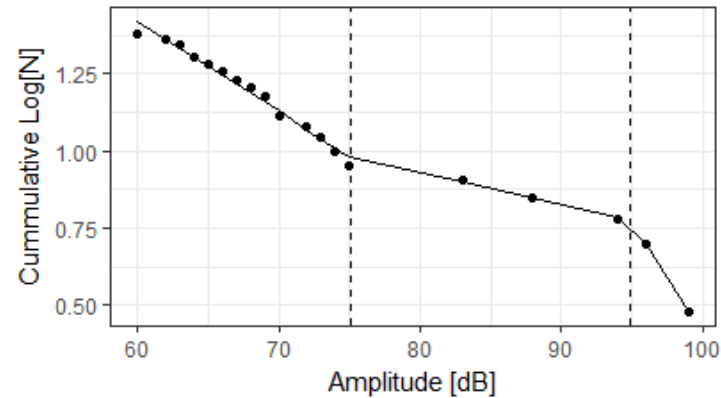

Strand 15

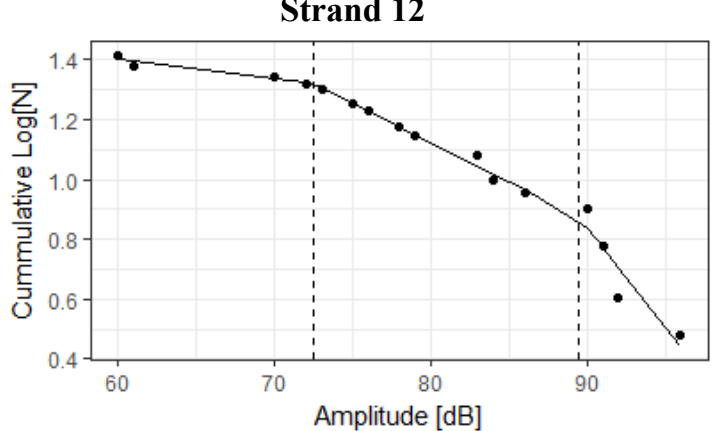

Strand 14

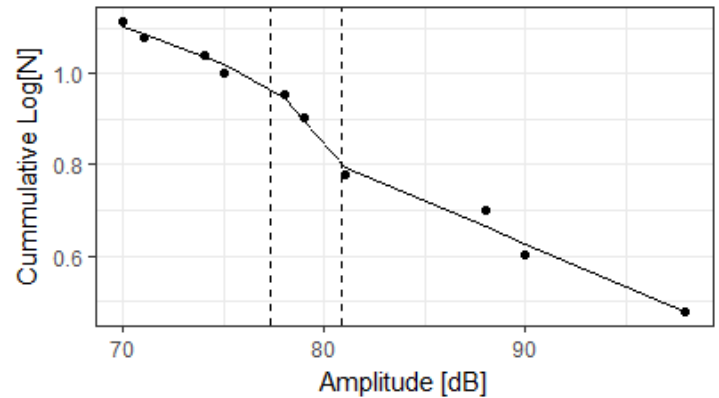

Strand 16 

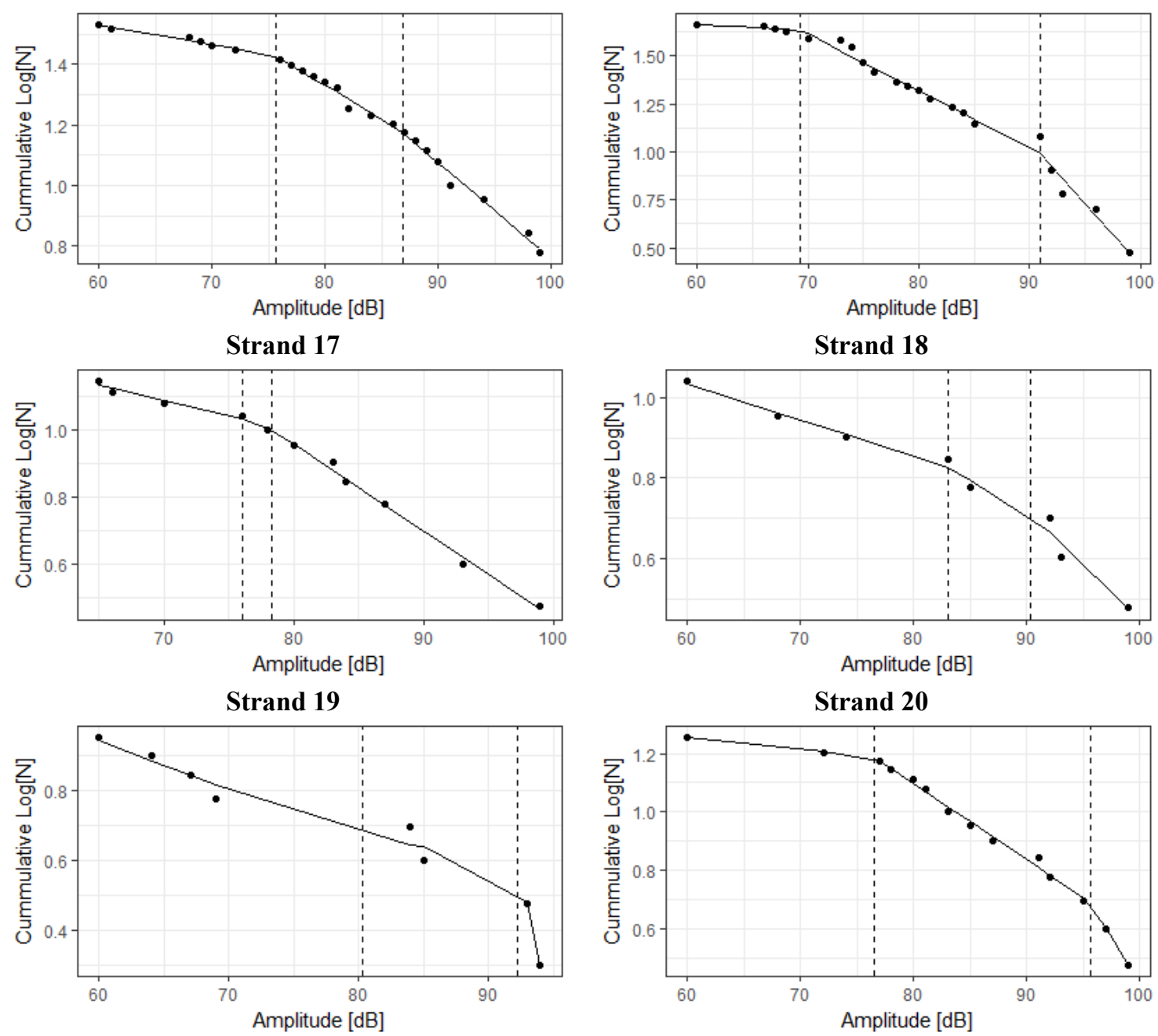

Strand 21

Strand 22

Figure 5.6. B-value regressions of each pre-tensioning strand for detensioning of a NEBT using a 3D sensor array

These b-values are plotted versus their respective pre-tensioning strand in Figure 5.7 and show an average b-value of 0.509 with seven b-values being significantly below the average and indicated below the red line. There were seven observed cracks during this test and seven b-values that were significantly below the average; however, additional comparative data sets would be required to verify a correlation. 


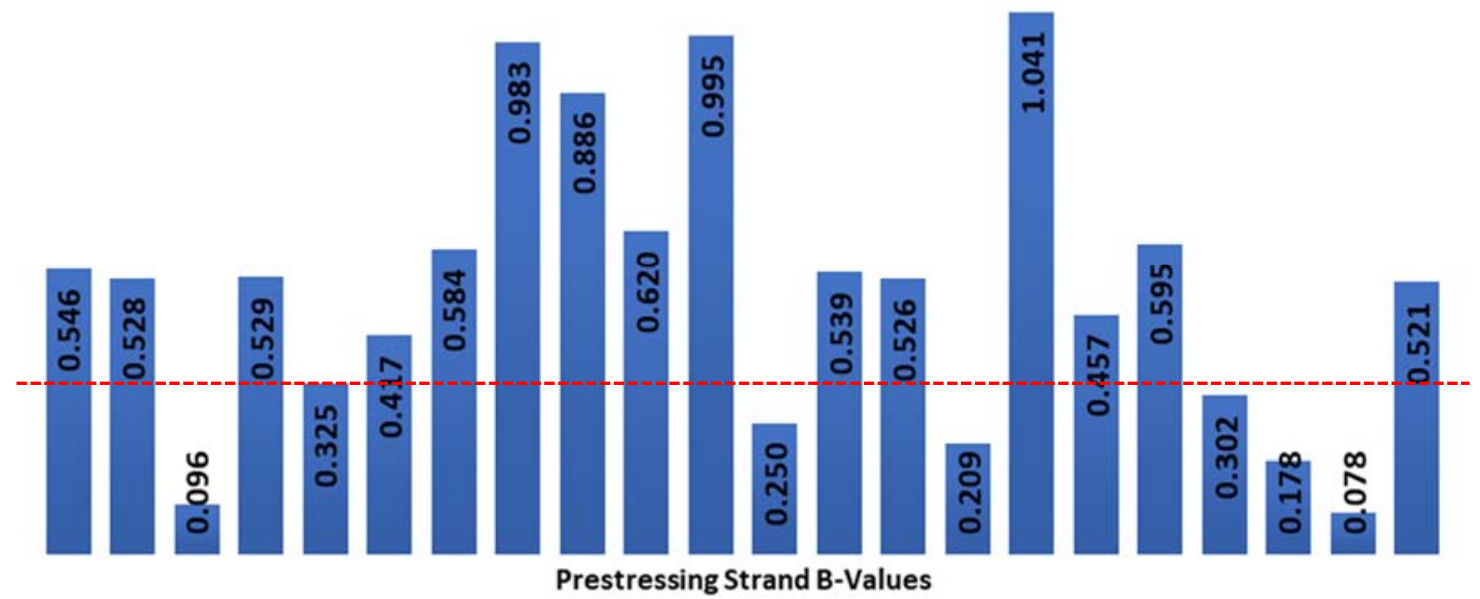

Figure 5.7. B-value of each pre-tensioning strand for detensioning of a NEBT using a 3D sensor array

Upon completion of detensioning, overhead tracked gantry cranes lifted the beams from the form decks and placed them roughly 50 feet (15 meters) away for storage and inspection. This process changes the geometric shape constraints on the beam and alters the internal load paths as it breaks loose from the form deck and rests on the two load point pairs of the gantry crane. Similar to the NEXT beam, the NEBT beam appeared to slowly camber over roughly a minute due to the highly tensioned embedded steel. The cambering process has the potential for creating areas of concentrated stress/strain leading to cracking. The instruments recorded only $12 \mathrm{AE}$ events during the craned onsite relocation from form to storage/inspection area. Again, if any cracking occurred, it was most likely near the center which would not be captured with the sensor array located near the ends of the beam.

Bulkhead removal produced 383 AE events. The bulkhead is the end of the formwork that serves the dual purposes of containing the uncured cement as well as providing patterned openings for the reinforcing bar and pre-tensioning strands to pass 
through to the deadman anchors on either end of the NEBT. Following movement of the beam to the storage/inspection location, a manual process using prybars and hammers removes the bulkhead. Although bulkhead removal created many AE events, they were largely a result of hammering. The cracks observed from detensioning did not grow during bulkhead removal.

\subsubsection{NEBT 2-D Sensor Array Data Collection}

The second sensor configuration placed eight sensors in a 2-D array, with all eight sensors located on one side of the web of the NEBT, within the end zone transfer depth, estimated as 60 times the diameter of the pre-tensioning strands or 36-inches $(914 \mathrm{~mm})$ per 5.11.4.1 of AASHTO Bridge Design Specifications (PCINE-14-ABC, 2014). The intent of the sensor array placement was twofold; 1) to focus AE data collection in an area predicted to have the greatest stress/strain transfer during loading from the release of the torch cut pre-tensioning strands, and 2) to use an equilateral triangular grid that helps to optimize the performance of the triangulation algorithm (ASTM 3100-17, 2017). The sensor coupling method and end zone reinforcement were the same as in the first sensor configuration.

The detensioning process produced 960 recorded AE events, as illustrated in Figure 5.8. The detensioning process was similar to that of the first NEBT 3-D sensor array test, however, the NEBT tested with the 2-D planar array was a hammerhead (haunched) section with a deep bottom flange that increased in depth at the middle to meet a pier cap. The hammerhead sections include a different layout of reinforcement as well as pre-tensioning strand layout as depicted in Figure 5.8. The recorded AE events tended to occur within the end zone transfer depth and were concentrated towards the interior ends of the observed 
end zone cracking. The 2-D planar sensor array that formed a pattern of near-equilateral triangles showed a noticeable reduction in the conglomerates of events located directly at the sensor locations on the surface of the NEBT as was observed with the first 3-D sensor array. Visual observations detected and documented end zone cracking immediately after the completion of the detensioning process as seen in Figure 5.8.b. End zone cracks ranged in length from 5-inches to 17 -inches (127 mm to $432 \mathrm{~mm}$ ). 


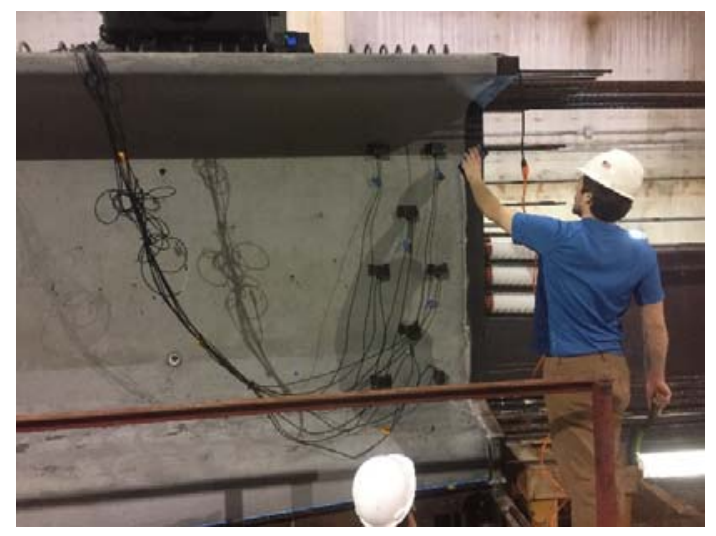

(a)

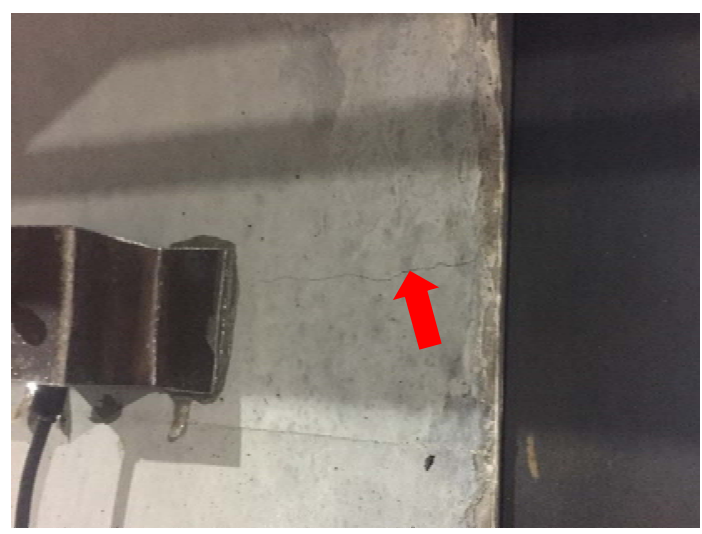

(b)

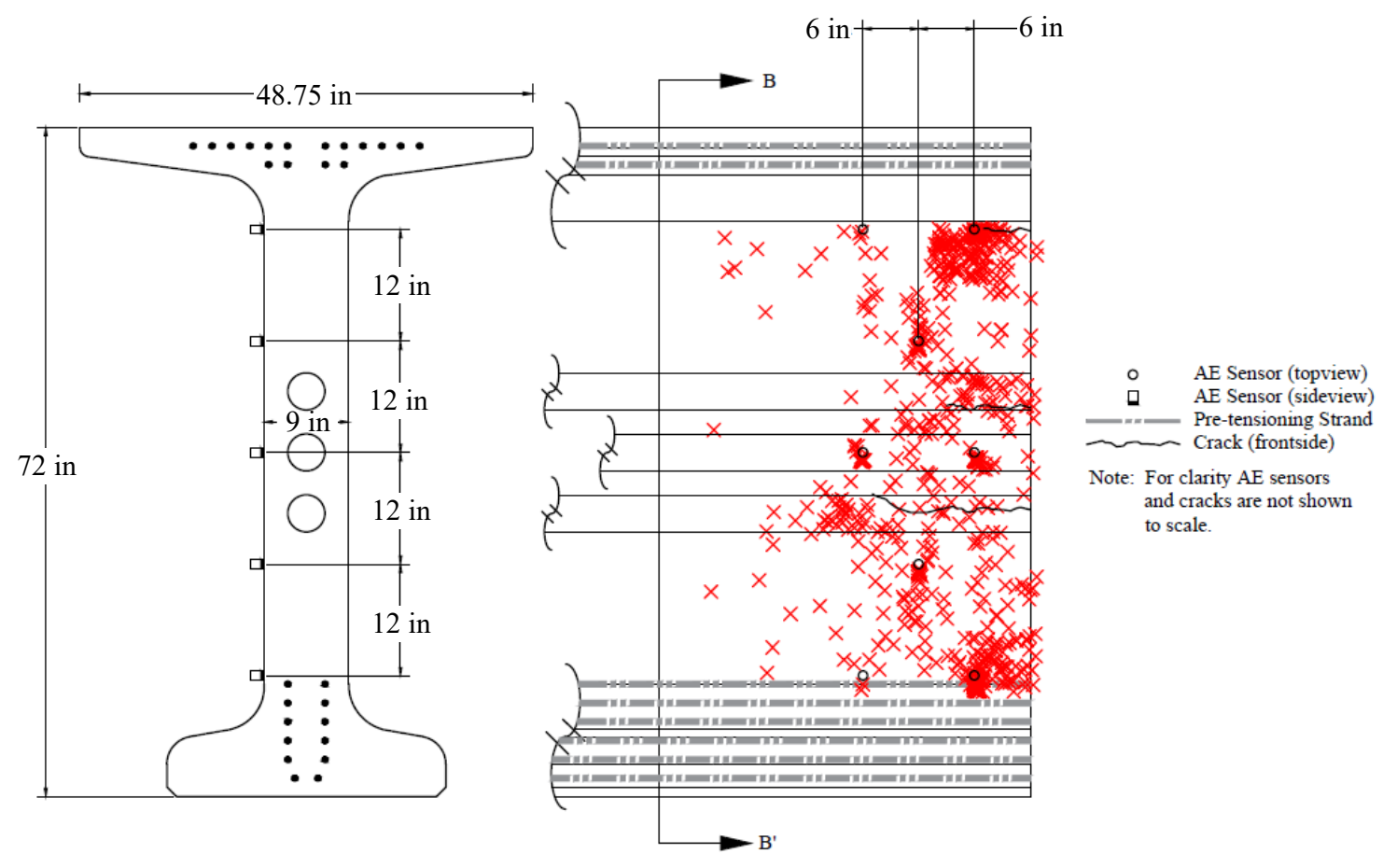

(c)

(d)

Figure 5.8. Hammerhead NEBT girder 2D sensor array field testing of detensioning with 960 recorded events (a) AE equipment/sensor setup, (b) observed horizontal web crack, (c) section view of NEBT girder, and (d) profile view of NEBT girder

Similar to the 3-D sensor array, recorded amplitude distributions of detensioning with a 2-D sensor array between the threshold lower limit (60 dB) and upper limit (99 dB) indicate large concentrations of $\mathrm{AE}$ events at the lower and upper amplitude limits but with a near normal distribution of AE events between these limits (Figure 5.9). 


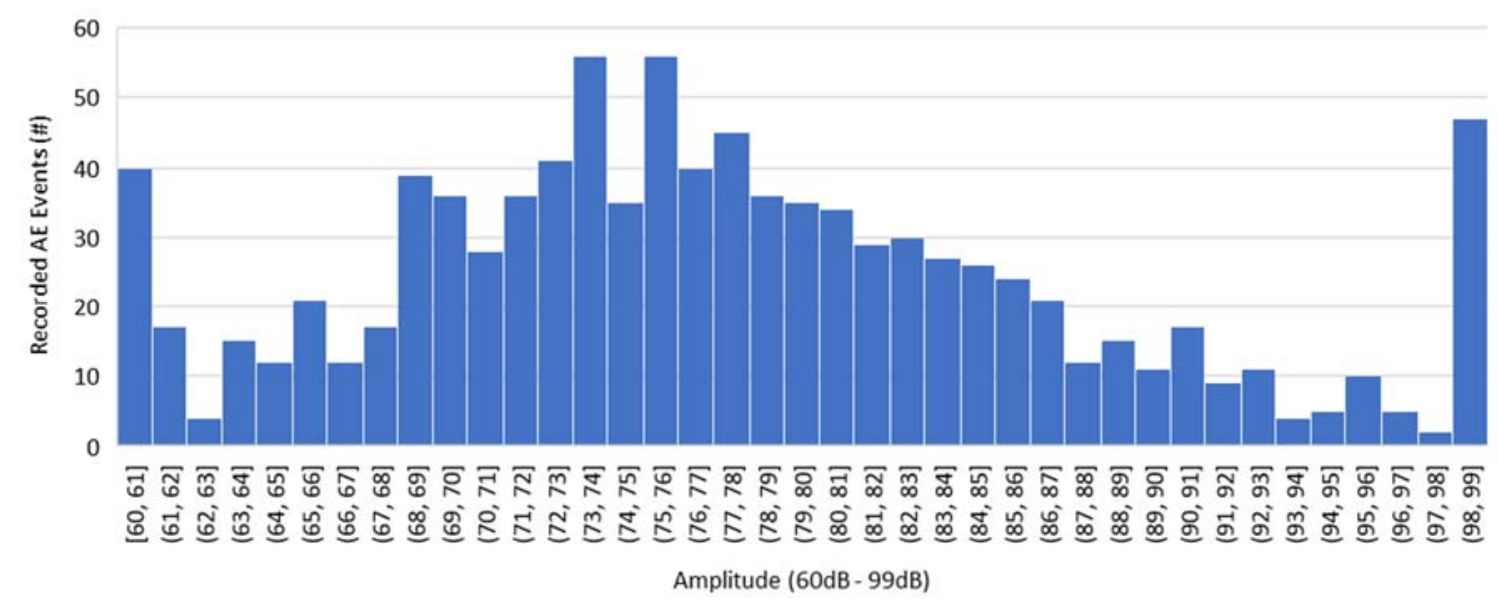

Figure 5.9. Amplitude distribution for NEBT girder 2D sensor array field testing of detensioning with 960 recorded events

There were a total of 28 pre-tensioning strands that were torch cut during the AE data collection using a 2-D sensor array. Similar to the 3-D sensor array, the AE data collected during detensioning were analyzed by plotting the number of AE events recorded over time and indicates 28 separate spikes in AE activity that correspond to a respective torch cutting event of a pre-tensioning strand as shown in Figure 5.10.

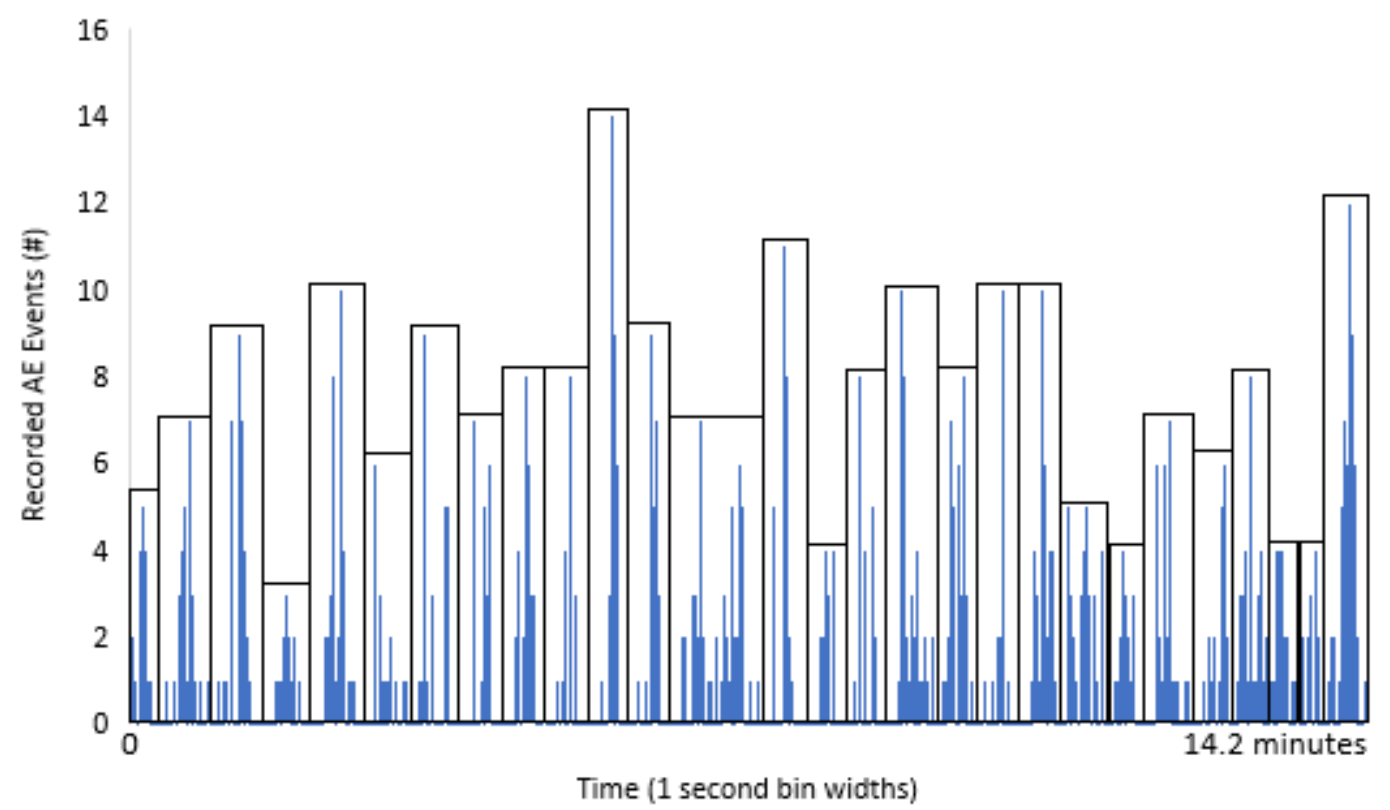

Figure 5.10. NEBT girder 2D sensor array field testing of detensioning with 960 recorded events over time with delineation of recorded $\mathrm{AE}$ events to each torch cutting of a pre-tensioning strand 
Similar to the 3-D sensor array, although the loading of the NEBT girders during detensioning is irregular by the varying loading location, the b-value analysis can be applied by analyzing the recorded $\mathrm{AE}$ events associated with each torch cutting of a respective pre-tensioning strand. Applying the b-value analysis, low b-values could indicate the occurrence of damage.

The b-value regression plots by pre-tensioning strand are shown in Figure 5.11.
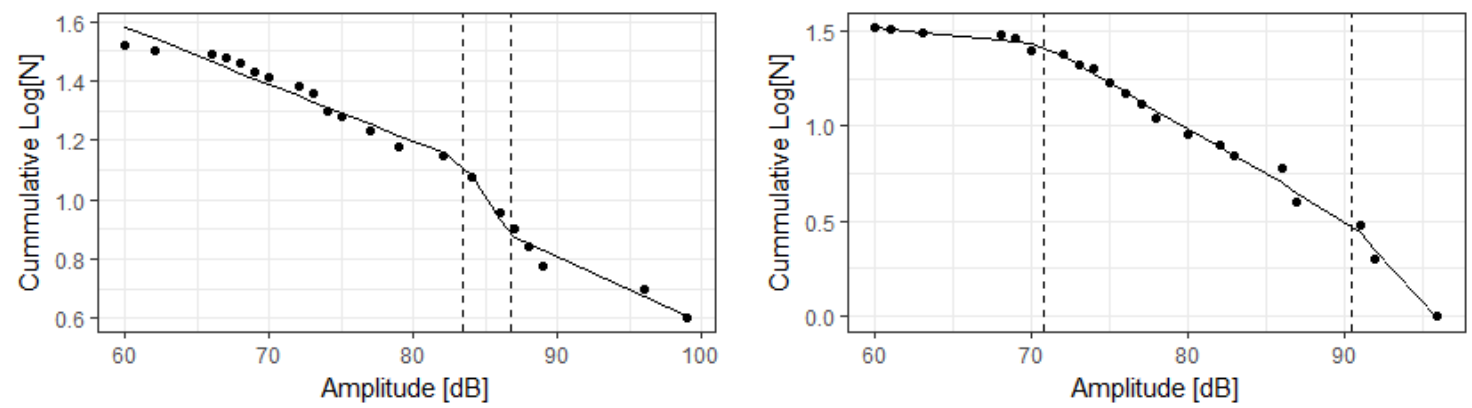

Strand 1

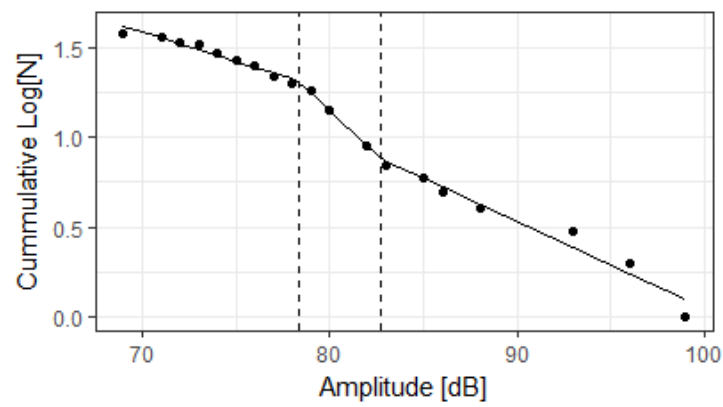

Strand 2

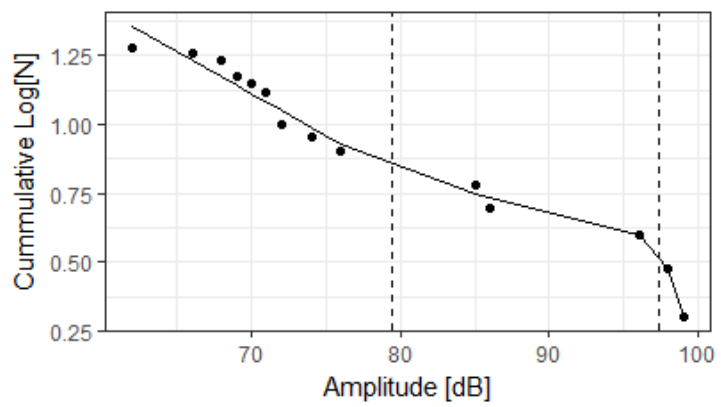

Strand 3

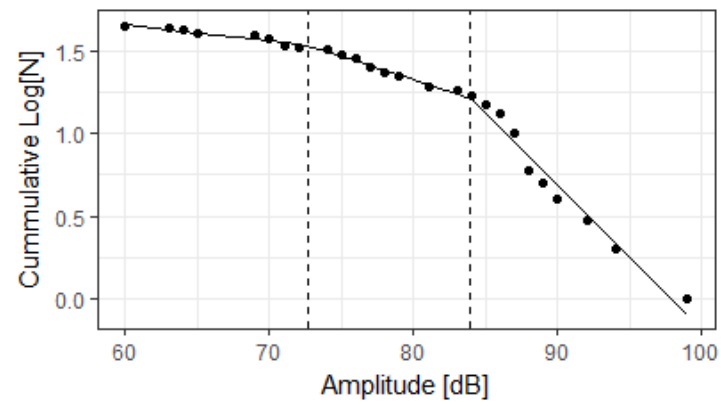

Strand 5

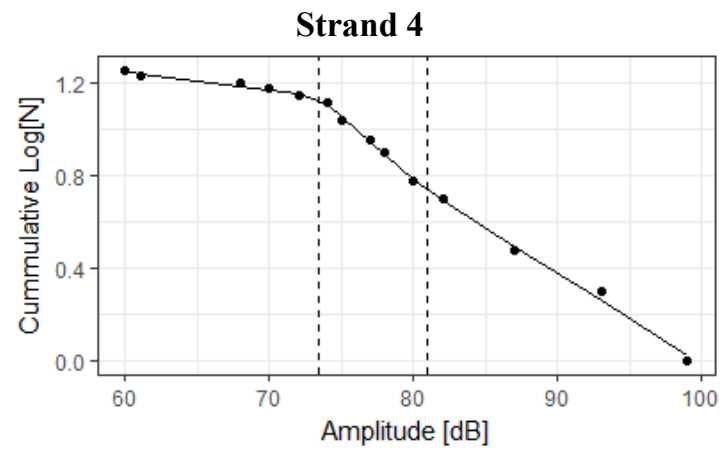

Strand 6 


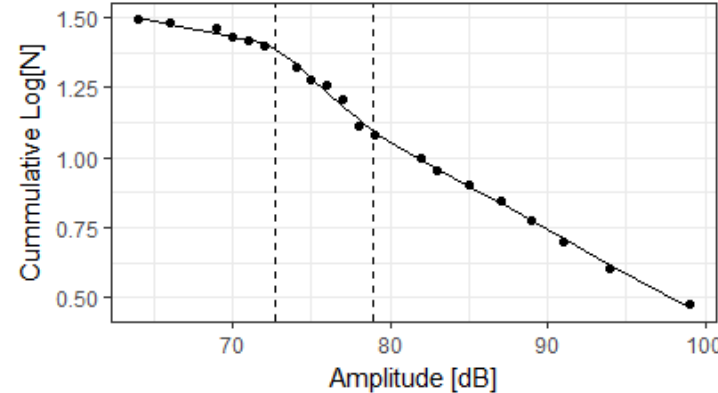

Strand 7

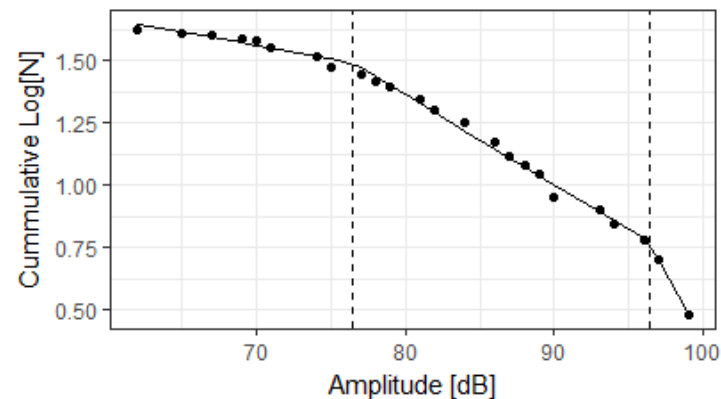

Strand 9

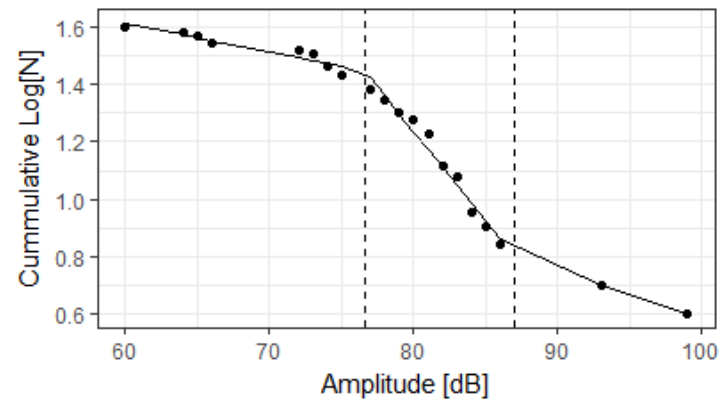

Strand 11

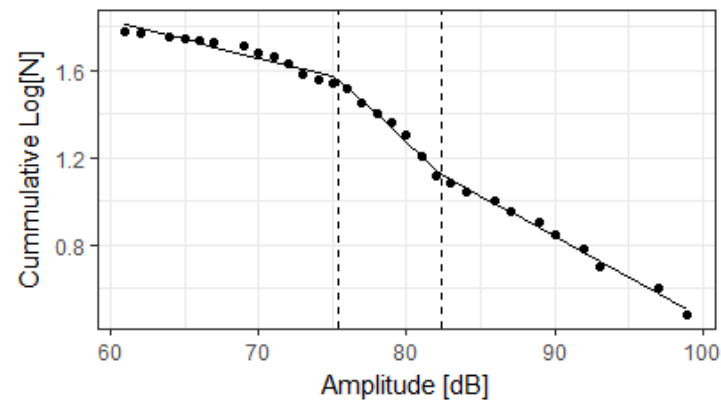

Strand 13

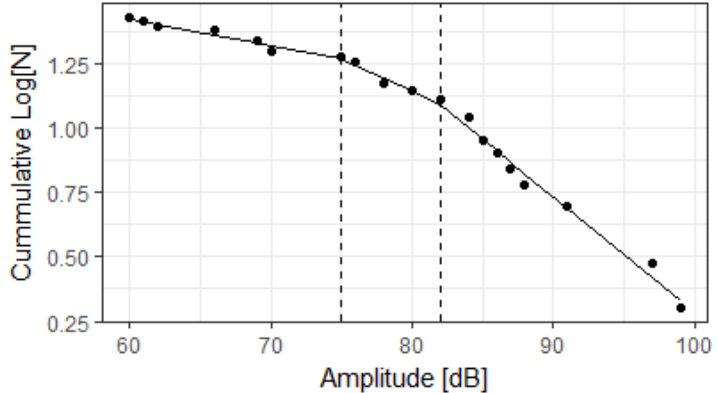

Strand 8

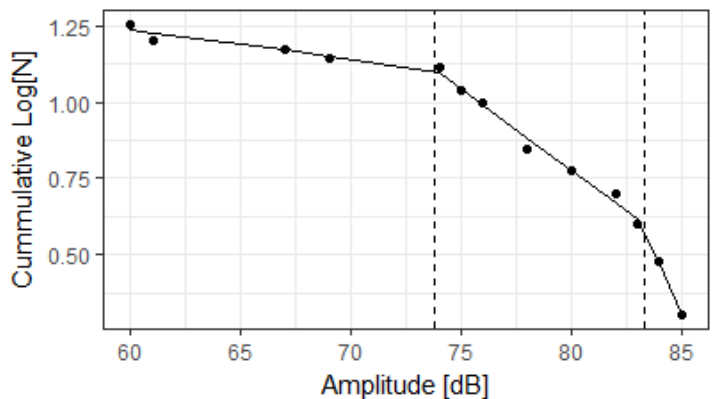

Strand 10

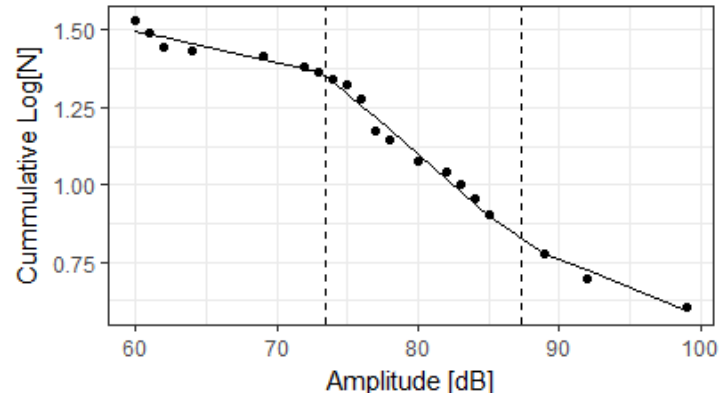

Strand 12

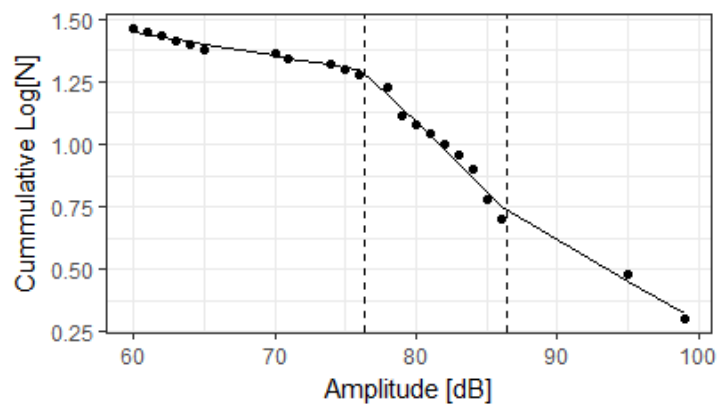

Strand 14 

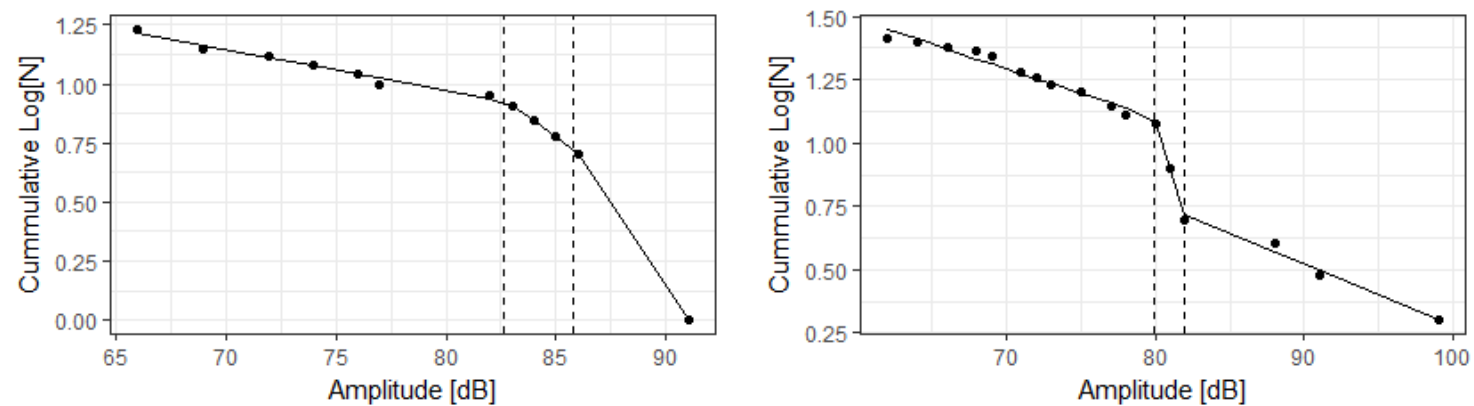

Strand 15
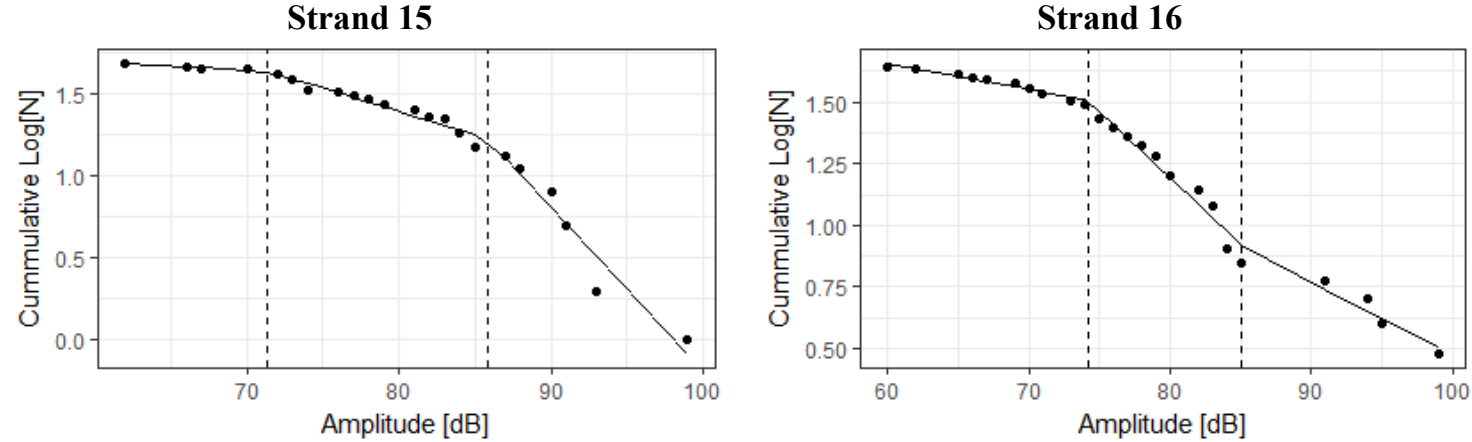

Strand 17
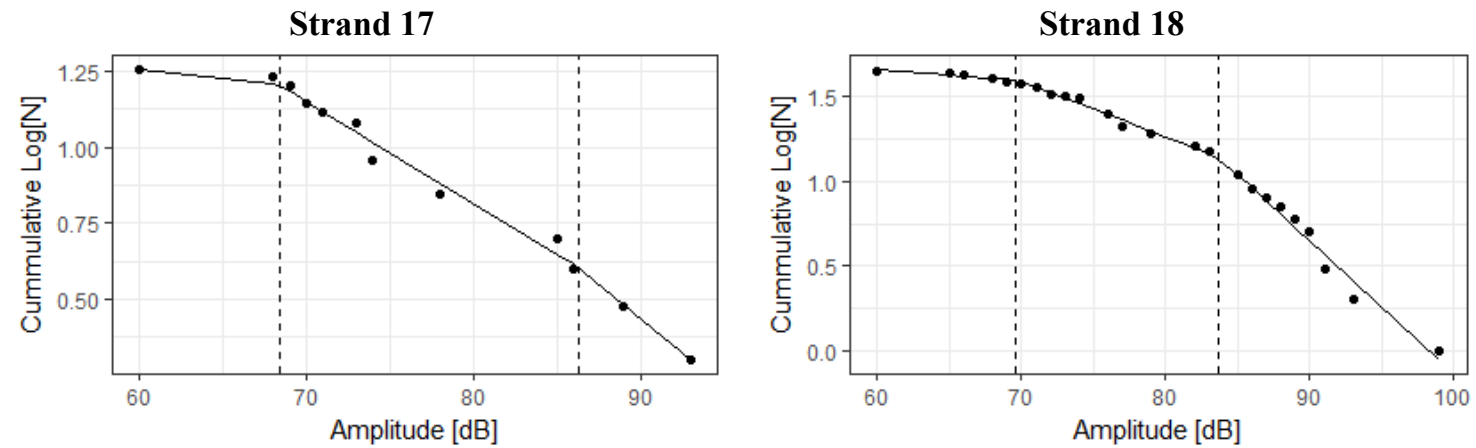

Strand 19

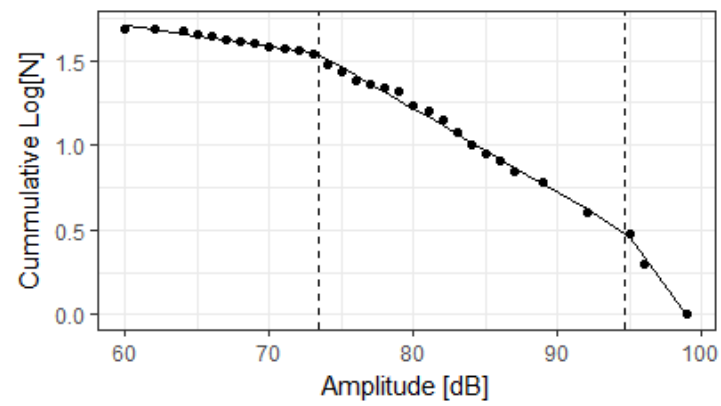

Strand 21

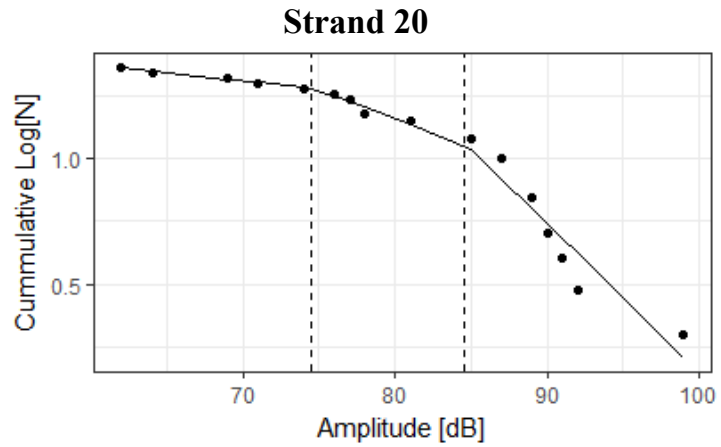

Strand 22 

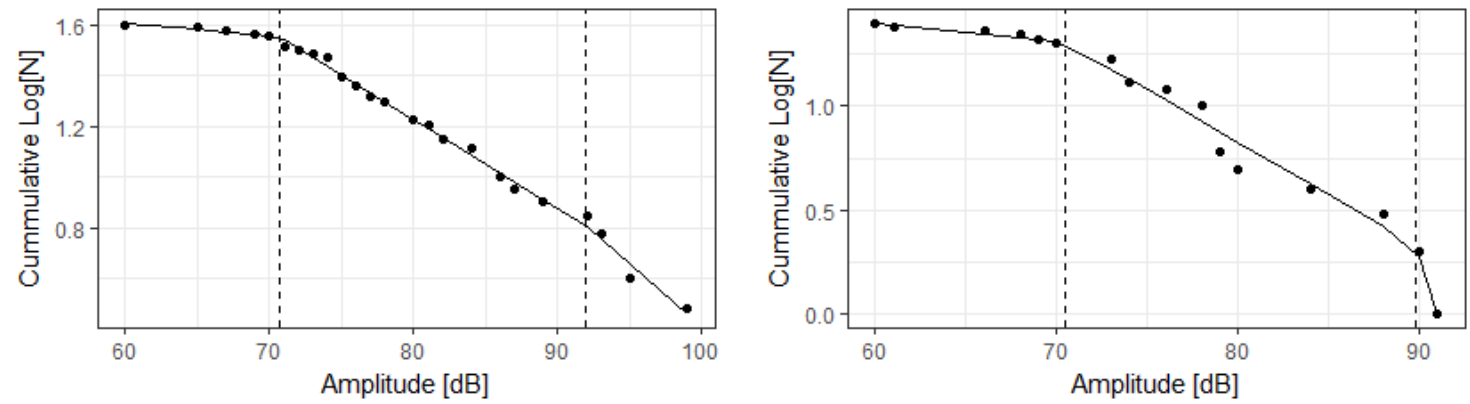

Strand 23
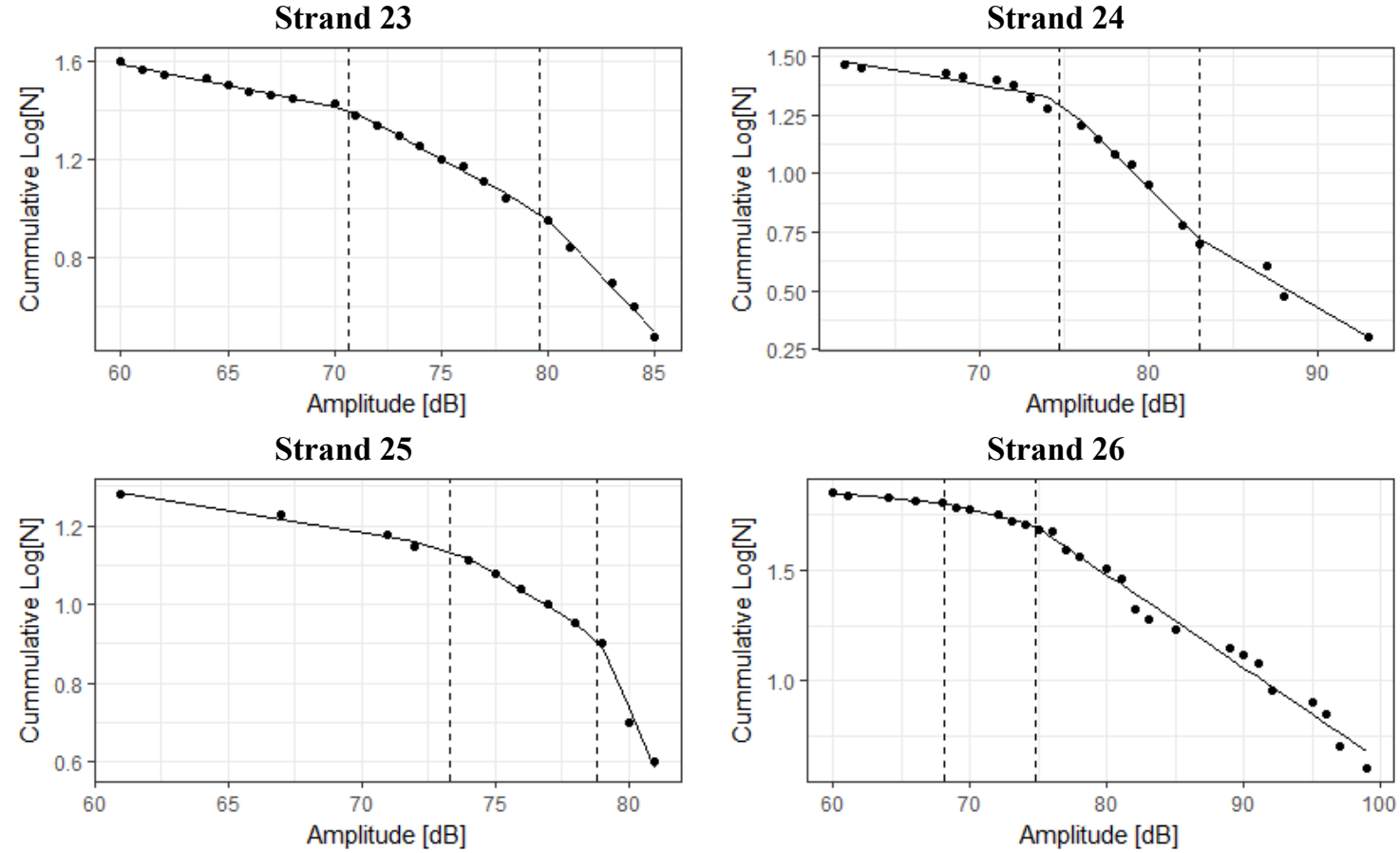

Strand 27

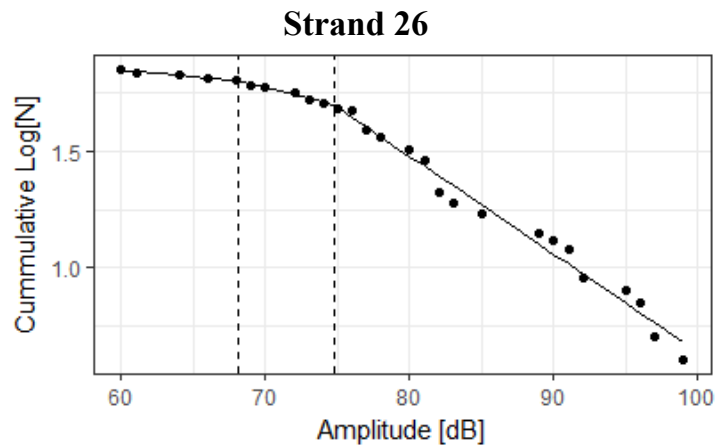

Strand 28

Figure 5.11. B-value regressions of each pre-tensioning strand for detensioning of a NEBT using a 2D sensor array

These b-values are plotted versus their respective pre-tensioning strand in Figure 5.12 and show an average $b$-value of 1.026 with ten $b$-values being significantly below the average and indicated below the red line. There were six observed cracks during this test and ten b-values that were significantly below the average; however, additional comparative data sets would be required to verify a correlation. 


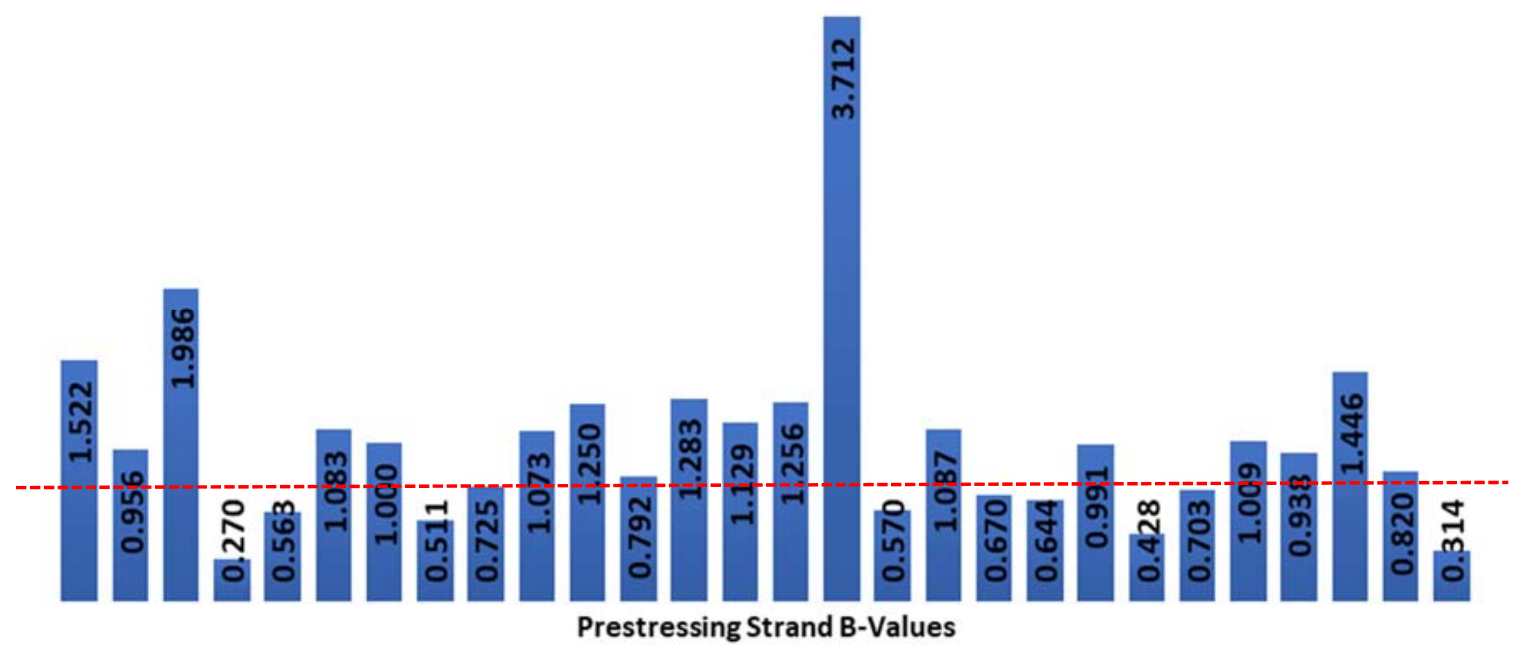

Figure 5.12. B-value of each pre-tensioning strand for detensioning of a NEBT using a 2D sensor array

Upon completion of detensioning, overhead tracked gantry cranes lifted the beams from the form decks and placed them roughly 50 feet (15 meters) away for storage and inspection. This process changes the geometric shape constraints on the beam and alters the internal load paths as it breaks loose from the form deck and rests on the two load point pairs of the gantry crane. The cambering process has the potential for creating areas of concentrated stress/strain leading to cracking. The instruments recorded only 16 AE events during the craned onsite relocation from form to storage/inspection area. Again, if any cracking occurred, it was most likely near the center which would not be captured with the sensor array near the ends of the beam. Observations of the girder found little, if any, camber occurring when lifting the girder out of the form bed.

During this 2-D planar sensor array test bulkhead removal data were not collected due to timing limitations between detensioning and removal of the bulkhead. 


\subsection{Conclusions from the Testing Results of Detensioning and Lifting}

The results included in this chapter demonstrated the viability of AE sensing in detecting and locating cracks in prefabricated pre-stressed concrete girders used as PBES in rapid bridge construction, which could potentially be used as a $\mathrm{QA} / \mathrm{QC}$ technique. $\mathrm{AE}$ field data collected from the NEBT showed more recorded AE events during the torch cutting of pre-tensioning strands than for the same process on the NEXT beam. This could be due to the thin web with three hollow post-tensioning tubes and dense packing of prestressing strands guiding the AE waves and reducing attenuation within the NEBT girder. A key observation made during the detensioning processes was the appearance of horizontal cracks in the web of the NEBT girder. The crack lengths ranged between 5inches and 20 -inches $(127 \mathrm{~mm}$ and $508 \mathrm{~mm}$ ) with vertical spacing between cracks ranging from 1.4-inches to 32 -inches ( $35 \mathrm{~mm}$ to $813 \mathrm{~mm}$ ), Table 5.1 .

Table 5.1. Quantification of AE events versus number of observed cracks during detensioning and craned movement

\begin{tabular}{lccc}
\hline Beam type & $\begin{array}{c}\text { \# of observed } \\
\text { AE events during } \\
\text { detensioning }\end{array}$ & $\begin{array}{c}\text { \# of observed AE } \\
\text { events during craned } \\
\text { form removal }\end{array}$ & $\begin{array}{c}\text { \# of observed cracks } \\
\text { (crack lengths) }\end{array}$ \\
\hline NEXT beam & 38 & 20 & 0 \\
Straight NEBT & 466 & 12 & 7 (5 inches-20 inches) \\
Haunched NEBT & 960 & 16 & 6 (5 inches-17 inches) \\
\hline
\end{tabular}

The observed end zone cracking occurred both within and outside of the region directly covered by the AE sensor array for the NEBT girders and AE event location distributions indicated differences in between in the 3-D and 2-D sensor arrays.

$\mathrm{AE}$ event data collected from multiple fabrication processes including the detensioning of pre-tensioning strands embedded in NEXT beams and NEBT girders yielded internal $\mathrm{AE}$ events indicative of crack nucleation. AE events recorded above the 
$60 \mathrm{~dB}$ amplitude pre-filter determined through laboratory testing may be indicative of internal cracking and provide an approximation of crack location. A statistical b-value analysis yielded some correlation between significantly lower b-values and the number of cracks observed; however, additional tests would be required to verify correlation. Additionally, the AE monitoring determined which fabrication process is responsible for the most $\mathrm{AE}$ events, which could point to the critical fabrication processes responsible for crack nucleation. This is highlighted by the NEBT girder field data that showed far more AE events occurred during the detensioning of the NEBT girder than during the craned removal from its form bed. 


\section{CHAPTER 6 ACOUSTIC EMISSION DATA COLLECTION AND RESULTS: TRANSPORT}

\subsection{Introduction}

This chapter describes a series of acoustic emission (AE) measurements taken on prefabricated and pre-stressed concrete bridge girders during transport from the fabrication facility to the bridge site. The girders were NEBT girders; fabricated at J.P. Carrara and Sons, Inc. in Middlebury, VT; and transported 160 miles (258 kilometers) to a bridge under construction in Rockingham, VT (Rockingham IM 091-1(66)). The testing began with observations of the transport process, followed by measurements on three girders - two straight and one hammerhead (haunch), running from June 14 to June 26, 2018. The measurement instruments consisted of an 8-channel array of AE transducers attached to the girders and connected to a central data acquisition unit strapped to the top of the girders and controlled via wireless telemetry by an operator in a chase vehicle. A preliminary analysis of the results indicated that the hammerhead girder produced significantly more AE events than the straight girders and transport maneuvers that tended to flex the girders by a differential change in elevation produced more events than traversing potholes. The girder movements did not produce any visible damage.

\subsection{Pilot Transport Tests}

The first transportation tests were a series of pilot tests to evaluate system configuration and operation. These pilot tests used two vehicles in a lead vehicle - chase vehicle configuration. The lead vehicle contained the AE test equipment and a small reinforced concrete block that specimened a large girder. The chase vehicle transported an operator with the system controller and a wireless connection to the lead vehicle. The tests 
ran on and around the University of Vermont campus grounds. The goals of the tests were:

1) To evaluate potential equipment modifications and system configurations required for transport testing; and 2) To evaluate the connectivity range of the wireless router during transport.

\subsubsection{Instrumentation Configuration}

The sensor configuration was with eight AE sensors in a 2-D array on the top surface of the concrete block of about 16.5-inch x 9.75-inch x 5.5-inch (419 mm x $248 \mathrm{~mm}$ x $140 \mathrm{~mm}$ ), Figure 6.1. The sensors pattern formed a grid of near equilateral triangles. The mounting arrangement secured the sensors into prefabricated steel U-shaped brackets with 5-minute epoxy securing the brackets to the concrete block, Figure 6.2. Custom electronic alterations to the equipment provided standalone power with a UPS remote power manager and remote monitoring with a wireless network connection. Additionally, a remotecontrolled electromechanical vibrator was secured to the concrete block with an adhesive tape, Figure 6.3. The purpose of the vibrator was to induce AE events during testing.

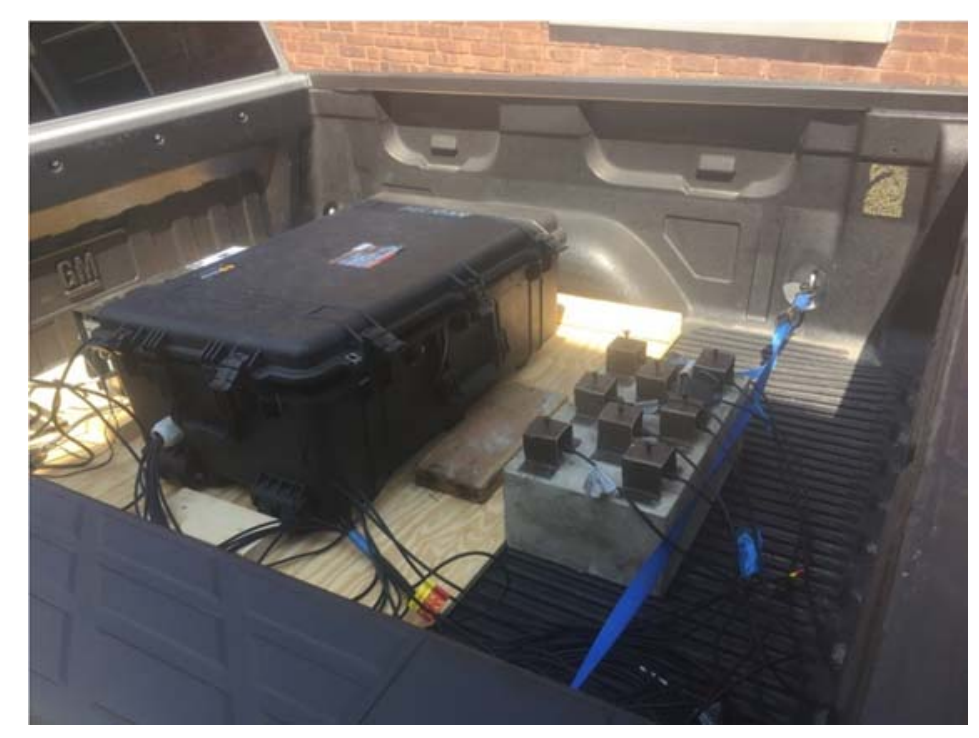

Figure 6.1. Pilot transport test $\mathrm{AE}$ equipment set-up 


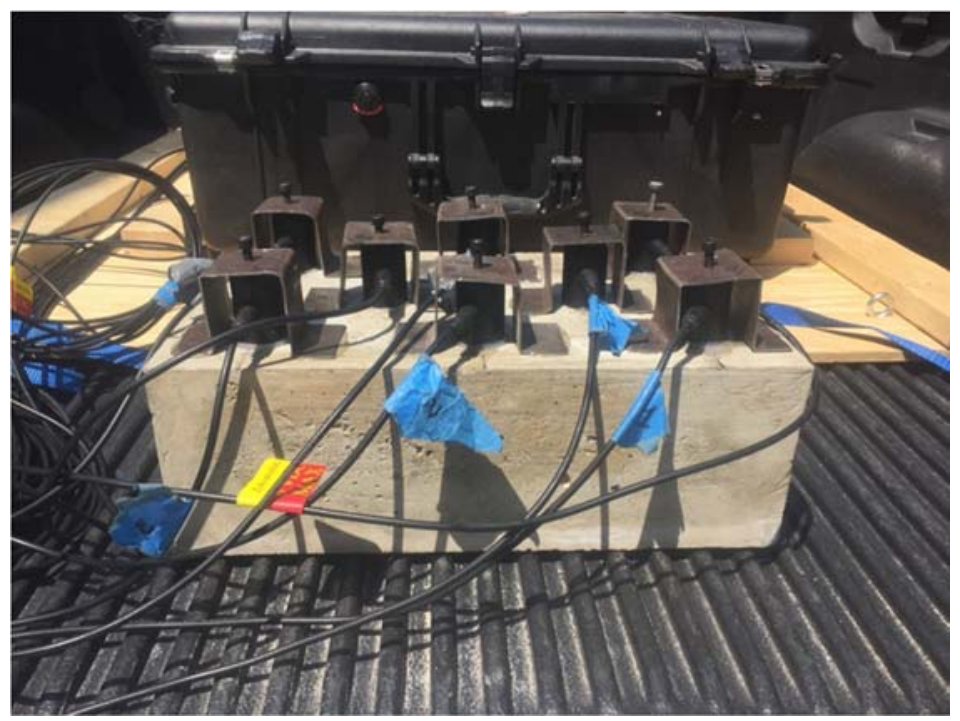

Figure 6.2. AE sensor connection to concrete block with $U$-shape brackets

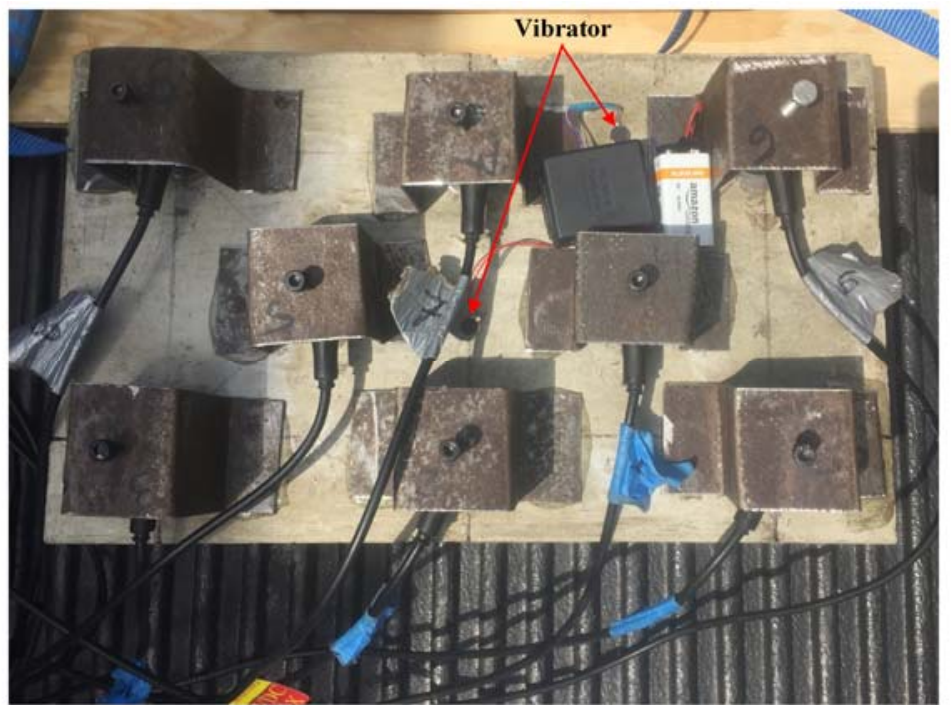

Figure 6.3. Pilot AE transport configuration showing concrete, electromechanical vibrator and transducer array, in bed of pickup truck

\subsubsection{Observations}

The overall results of the pilot test were positive. Road vibrations induced AE events in the small-scale concrete block. A tailing vehicle with a laptop computer serving as a remote controller followed the truck on a test drive to determine the range of 
connectivity of the wireless router and receiver from the lead to tailing vehicle with the wireless router. The tail vehicle stopped, and the transport truck continued until connectivity was lost. The measured viable telemetry distance was 645 feet (197 meters). From the pilot transport test, it was clear that the portable power source needed to be changed from the available UPS to batteries for increased power duration and that the remote telemetry configuration was capable of providing real-time updates of AE activity and for remote control of the test instruments during transport testing.

\subsection{NEBT Transport Observation}

\subsubsection{Transport Details}

The transport of NEBT girders began from their fabrication site at J.P. Carrara \& Sons, Inc. (Carrara) precast concrete plant located at 2464 Case Street, Middlebury, Vermont (Site) with the drop off location of the IM 091-1(66) I-91 bridge construction site in Rockingham, VT, as part of the bridge construction on I-91 I Rockingham, VT. The transport route was 160 miles (258 kilometers). Figure 6.4 shows the route, which followed the main highway (US 7) from Middlebury to Burlington, and then the interstate roads (I189, I-89 and I-91) from Burlington to Rockingham. The AE testing occurred during the transport of a NEBT girders throughout June 2018. 


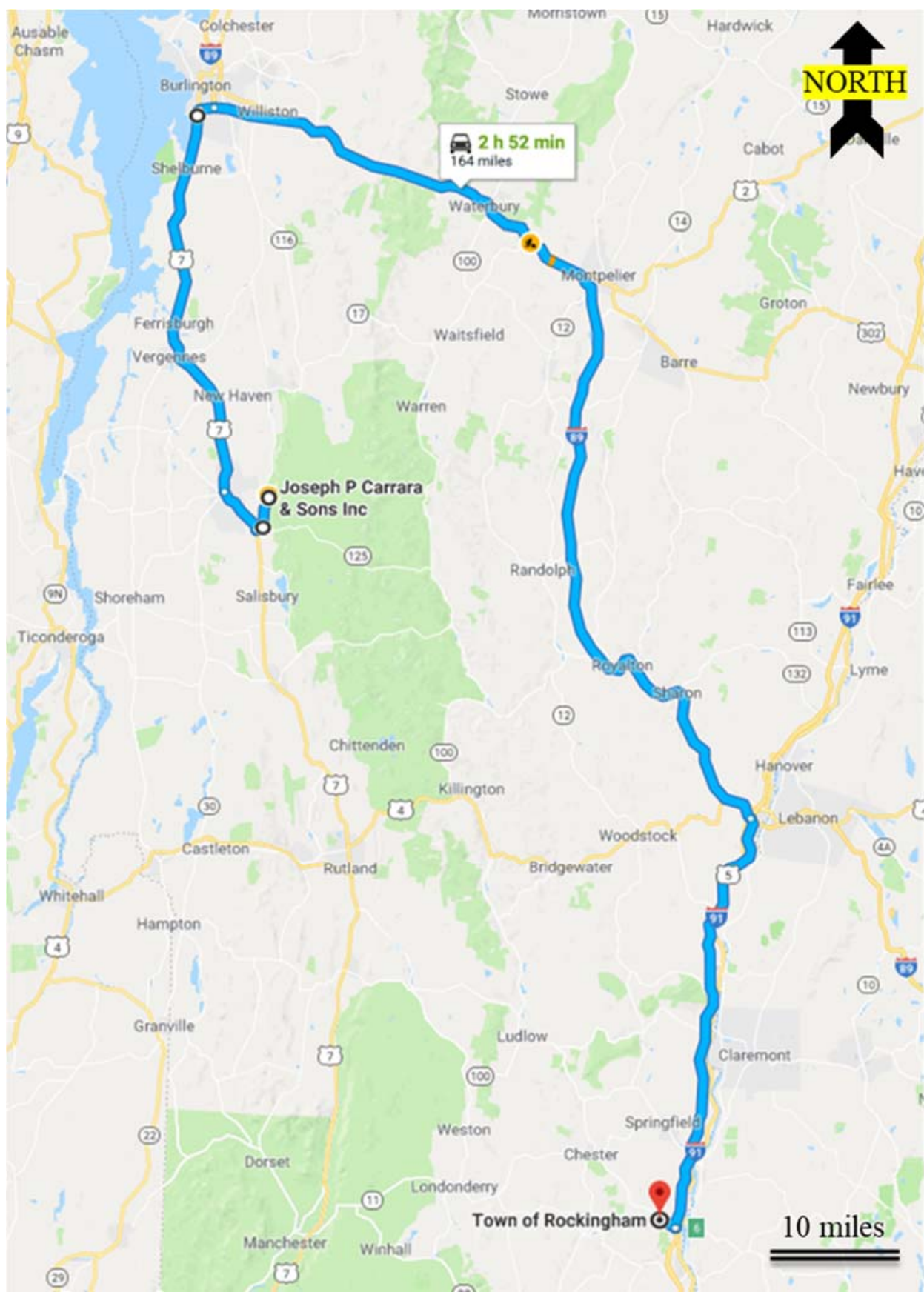

Figure 6.4. Travel route from J.P. Carrara \& Sons Inc.in Middlebury, VT to the IM 091-1(66) I-91 bridge construction site in Rockingham, VT (source: Google Maps)

\subsubsection{Transport Process Observations}

Transport of the NEBT girders was typically a 2-day process. The first day was at the fabrication facility and was for loading the girders onto the truck with gantry cranes. 
The second day began at the fabrication facility, followed by transport to the bridge site and then unloading at the bridge site. The truck used for transport was a jeep and dolly rig that allowed for independent steering of the rear dolly by a trailing chase vehicle that followed during transport. Fabrication drawings included details for placement of the NEBT girder on the jeep and dolly. A system of chains, rachets, and steel bars secured the NEBT girder to the jeep and dolly.

On the second day, departure from Carrara's started between 3:00am and 4:00am. The number of girders transported per day ranged between 1 and 3 with 15 -minute gaps between departures of each beam to allow for traffic flow between each convoy. The convoy for each girder consisted of a lead oversized vehicle escort, a lead police escort, the tractor with beam on a jeep, the rear steering vehicle (dolly), and finally a rear police escort. Each load required a permit and was required to abide by local restrictions as well as state restrictions for times and conditions at which oversized loads are allowed to travel. Travel was not permitted during rain, fog, or on wet roads. Additionally, travel was not permitted between 7:00am and 8:00am as well as between 12:00pm and 1:00pm. If no issues arose during transport the travel from Carrara's in Middlebury to the drop site in Rockingham, VT took approximately 4-hours for a hammerhead NEBT girder and approximately 3.5hours for a straight section NEBT girder. Once at the drop site, the tractors detached from the loads and reattached to the empty jeep and dolly from the previous day's load, to be brought back to Carrara' for loading.

Observations of the transport indicated that there were multiple factors of concern for usage and placement of the AEwin ${ }^{\mathrm{TM}}$ Sensor Highway III and associated sensors. These factors were: 1) safely securing equipment; 2) clearance constraints from overhead 
powerlines; 3 ) exposing the equipment to weather; 4) maintaining wireless connectivity to the AEwin ${ }^{\mathrm{TM}}$ Sensor Highway III; 5. safety during placement and removal of the AEwin ${ }^{\mathrm{TM}}$ Sensor Highway III and associated sensors.

\subsubsection{System Modifications}

The observation of the girder transport identified a set of issues that required modification to both the equipment and attachment procedures. An outdoor-rated steel case houses the AEwin ${ }^{\mathrm{TM}}$ Sensor Highway III data acquisition system. Two $12 \mathrm{~V}$ motorcycle batteries connected in parallel provided power to the AEwin ${ }^{\mathrm{TM}}$ Sensor Highway III and wireless router through modified electrical bus connections. Gorilla tape secured the wireless router to the AEwin ${ }^{\mathrm{TM}}$ Sensor Highway III steel housing. The placement of the steel housing was directly on top of the NEBT girder in the gap between exposed deck attachment rebars. 2-inch $(50 \mathrm{~mm})$ wide heavy duty rachet straps looped all the way around the NEBT girder secured the assembly in place. Overnight rain protection consisted of placing a waterproof back pack cover over the AEwin ${ }^{\mathrm{TM}}$ Sensor Highway III with wireless router and covering the AEwin ${ }^{\mathrm{TM}}$ Sensor Highway III and batteries with a tarp. The height of the placement required an extension ladder.

Attaching the AE sensors to the girder followed. The first step secured U-shaped brackets to the beam with quick-setting epoxy, followed by a 5 to 10 -minute curing cycle. The next step applied high-vacuum silicone grease to the sensor wear plates. Next, the sensors were placed in the U-shape brackets and secured using the set screw. The final step secured excess cable lengths to the exposed rebar with zip-ties on the top of the beam. 


\subsection{Straight NEBT End Zone Data Collection (Transport Test 1)}

The first NEBT transport test (TT1) collected AE data on the end zone of a straight NEBT girder. Data were collected on June 22, 2018.

\subsubsection{Equipment Layout}

The placement of the AEwin ${ }^{\mathrm{TM}}$ Sensor Highway III was directly on the top of the NEBT girder. The sensor arrangement was eight AE transducers in a 2-D array in the end zone (the end zone transfer depth is estimated as 60 times the diameter of the pre-tensioning strands or 36-inches (914 mm) per 5.11.4.1 of AASHTO Bridge Design Specifications) of the vertical web section of the NEBT girder. Since this particular girder was slated for placement on the outside of the deck, care was taken to place the transducers on an inwardfacing side. This placement reduced the visibility of any residual stains left by the epoxy used for attaching the AE transducers. The selection of the sensor array configuration was to match that used in a prior test of this study at the fabrication facility during detensioning and craned lifting. U-shaped brackets with set screws secured the sensors to the beam. Placing a tarp and waterproof back pack cover protected the equipment from rain. The equipment and covers remained in place overnight.

The first step the following morning was to remove the tarp and waterproof back pack covers and then to power on the equipment. The next step verified connectivity and performance with a tap test. Upon successful completion of the morning tests, an operator rode in the rear steer vehicle to stay close enough to the $\mathrm{AE}$ monitoring equipment and took notes during the transport test. 


\subsubsection{Representative Data Collection}

AE event monitoring of the end zone of a straight section of a NEBT proceeded over the course of roughly 160 miles (258 kilometers) in transport from the fabrication location to the installation location. The number of recorded AE events totaled 673. Figure 6.5 shows the spatial distribution. The AE events tended to occur in the upper half of the web section of the NEBT. An examination of the NEBT girder following transport found no new cracks and no new growth of the existing cracks. 


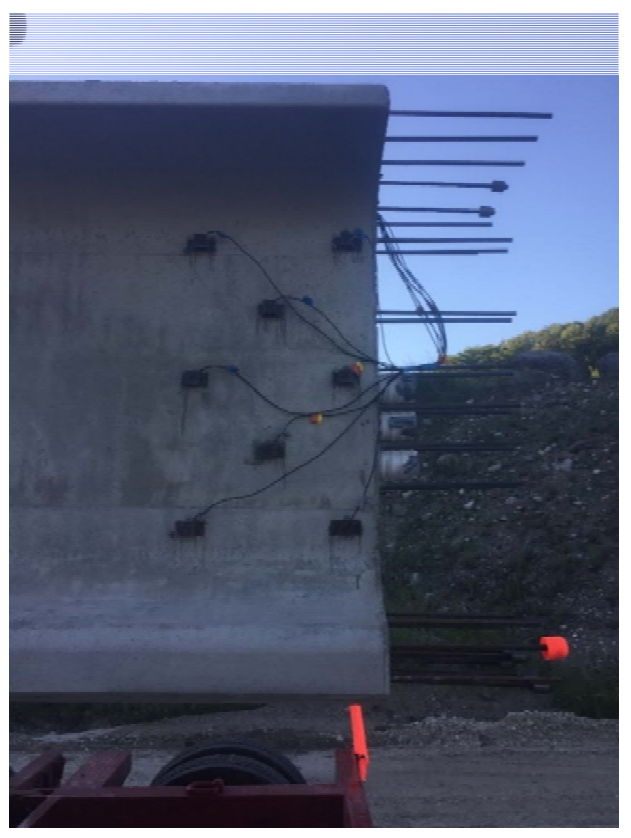

(a)

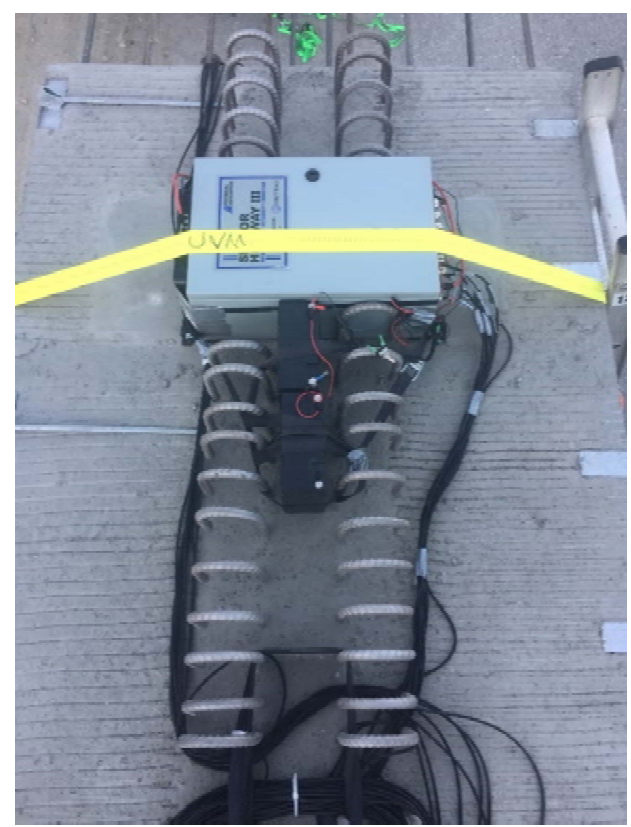

(b)

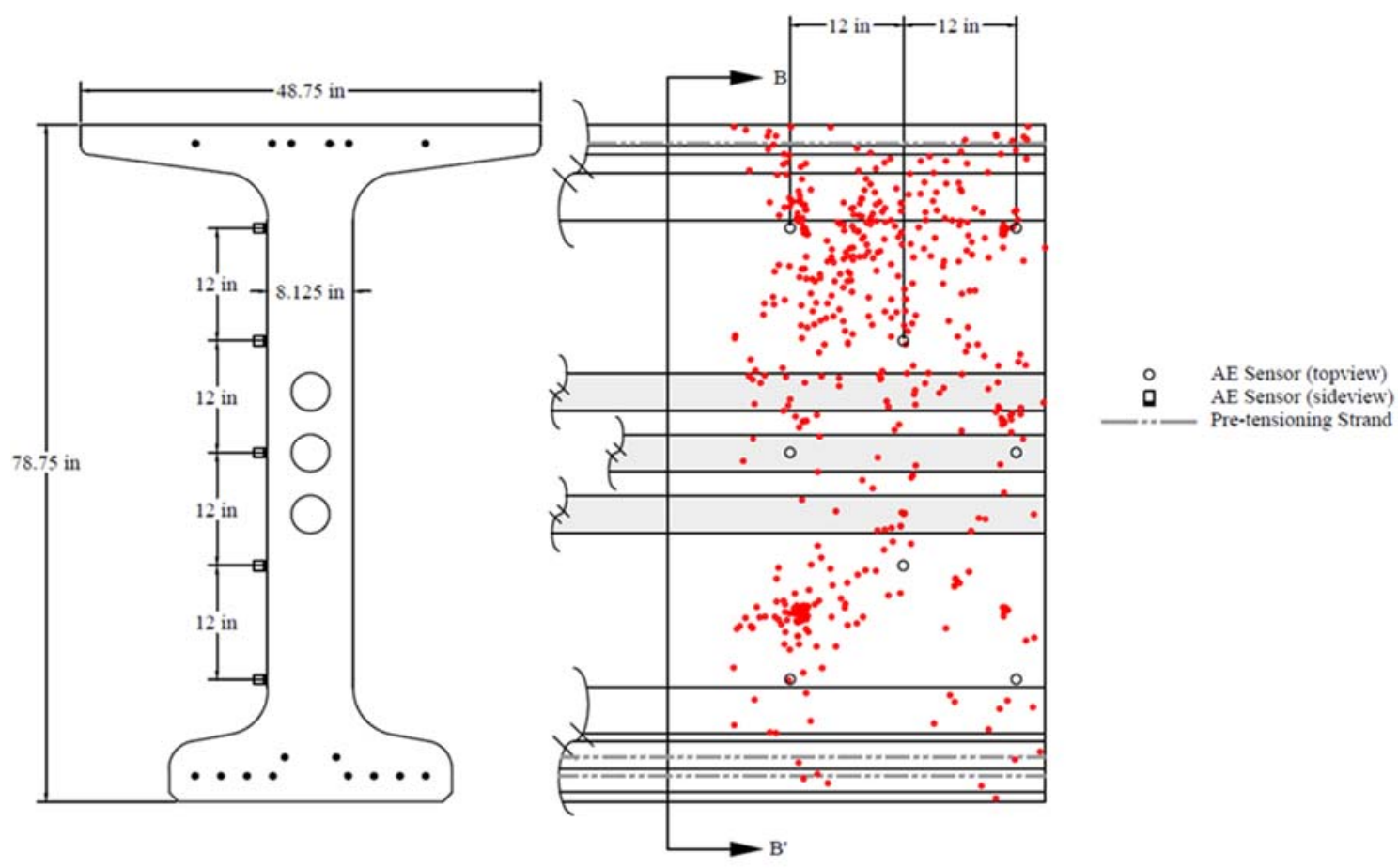

(c)

(d)

Figure 6.5. Transport Test 1 - AE measurements of the end zone vertical web of a straight NEBT during transport with 673 recorded AE events. (a) photograph of 2-D sensor array, (b) photograph of equipment set-up, (c) cross-section of NEBT tested, (d) profile view of NEBT including AE event locations, sensors, and observed cracks. 
The recorded AE events are depicted in Figure 6.5 were also plotted by amplitude as shown in Figure 6.6. The amplitude plot of AE events show a weak clustering pattern between $\mathrm{AE}$ events of different amplitudes but there appears to be small pockets of low amplitude AE event clusters near the top of the beam.
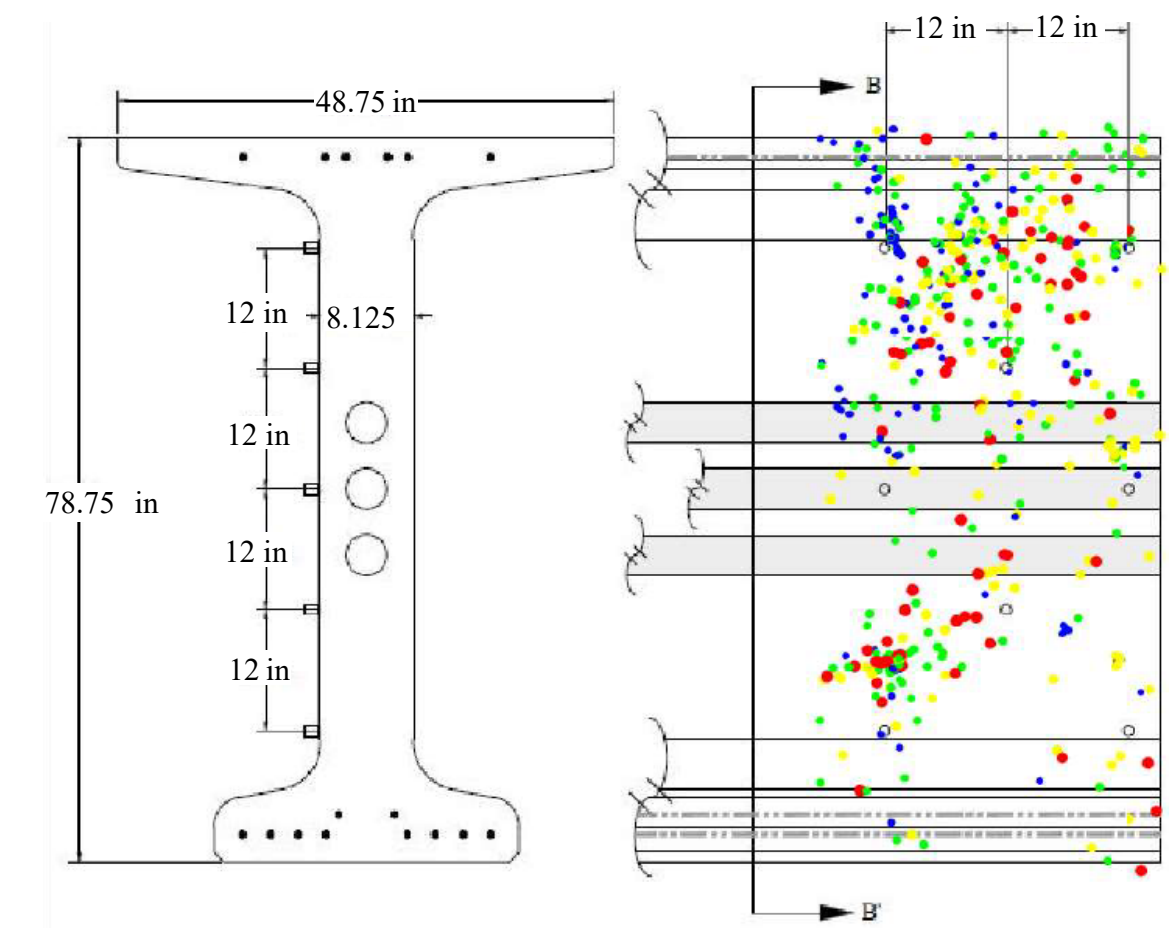

Blue: 60dB - 69dB Green: 70dB - 79dB Yellow: 80dB - 89dB Red: 90dB - 100dB

Figure 6.6. Transport Test 1 - AE measurements plotted by amplitude of the end zone vertical web of a straight NEBT during transport with 673 recorded $A E$ events.

Recorded amplitude distributions from the end zone of a straight NEBT during transport using a 2-D sensor array between the threshold lower limit $(60 \mathrm{~dB})$ and upper limit (99 dB) indicate a semi-normal distribution between these limits (Figure 6.7). 


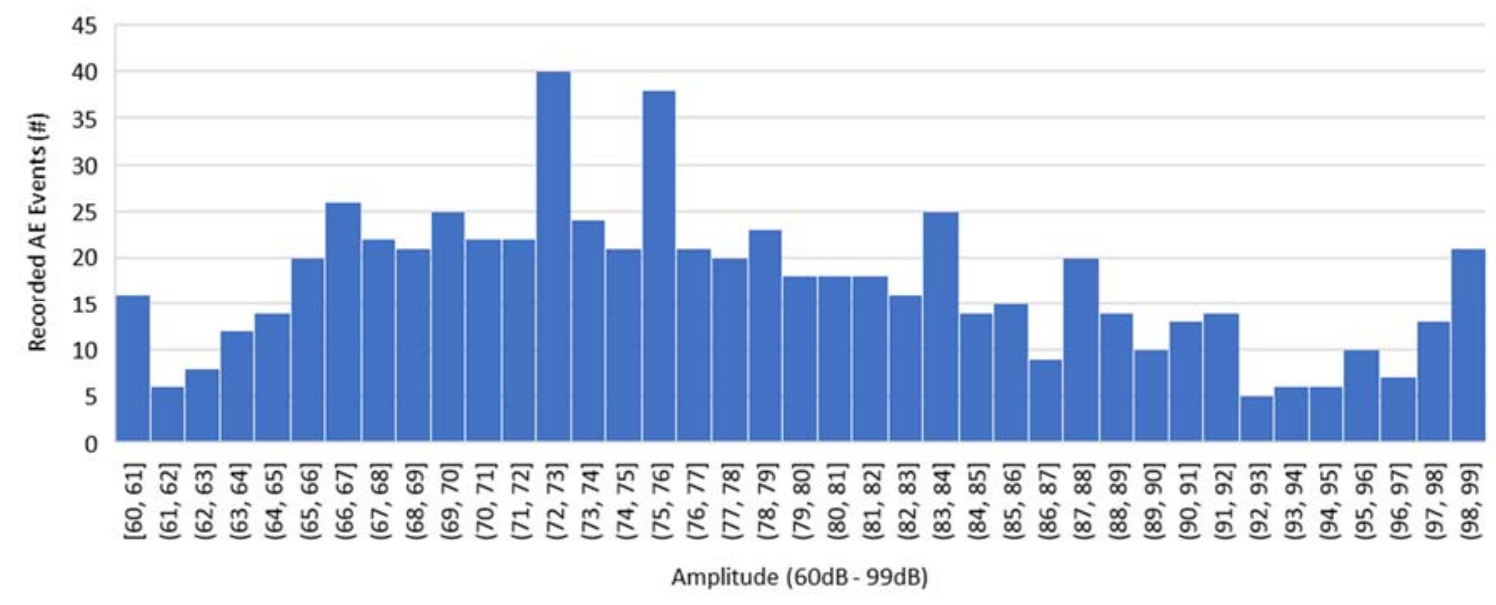

Figure 6.7. Amplitude distribution for a 2D sensor array transport test of the end zone of a straight section NEBT girder with 673 recorded events

$\mathrm{AE}$ event data collection during transport included the time history of data collection. The AE event time histories were then plotted (Figure 6.8) in 5-minute intervals with respect to three different conditions; secondary/highway, interstate, or stopped. The AE event time histories for TT1 indicate a greater level of AE event occurrences when on secondary/highway roadways compared to $\mathrm{AE}$ event data collection on the interstate. Additionally, no AE events were collected during stops. The decreasing trend in the number of $\mathrm{AE}$ events with time could be representative of a breaking-in period, i.e. the Kaiser effect, where AEs only occur if the maximum previous experienced stress is exceeded. 


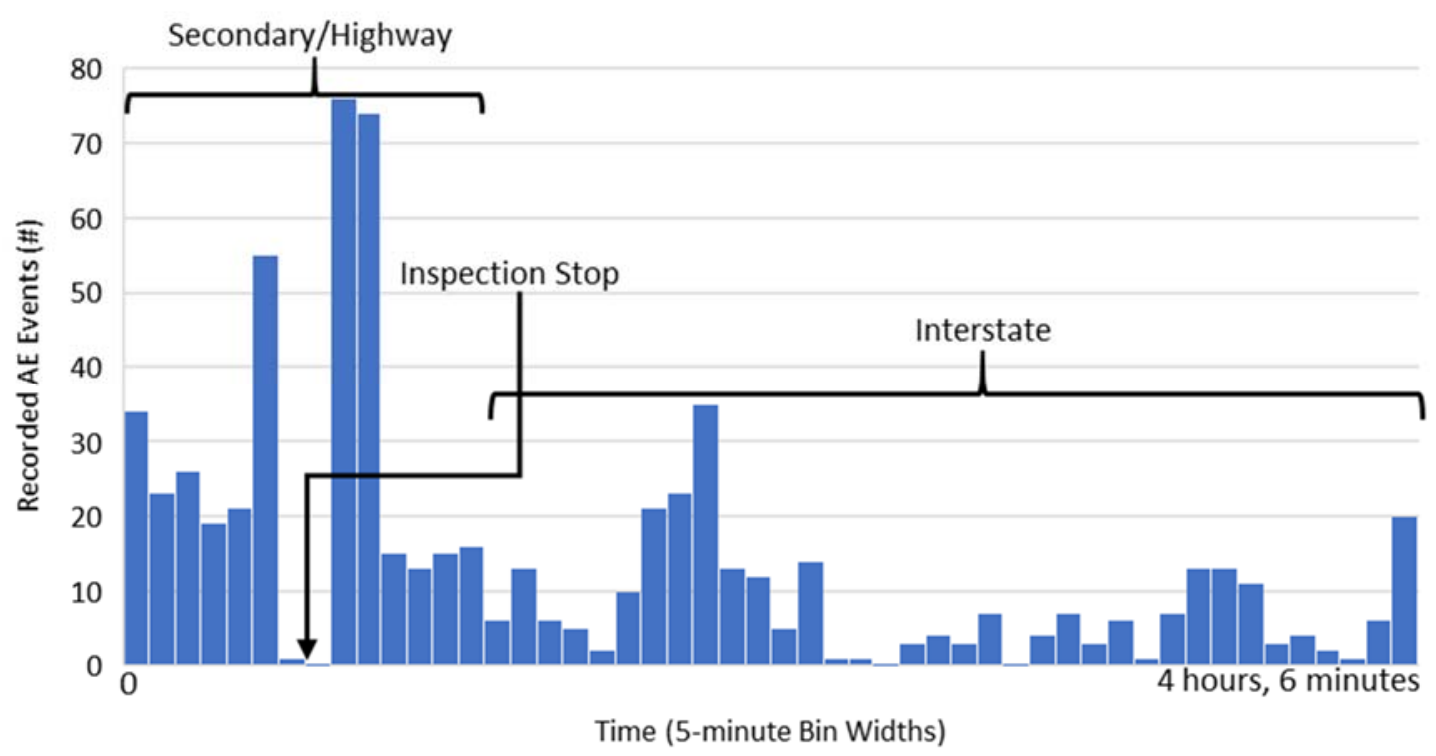

Figure 6.8. AE event density during transport testing of end zone region of straight NEBT, with a total event count of 623

Although the loading of the NEBT girders during transport is irregular, the b-value analysis can be broken up into time intervals where a drastic decrease in b-value could indicate the occurrence of damage.

The b-value regression plots in 30-minute increments of time during TT1 is shown in Figure 6.9.
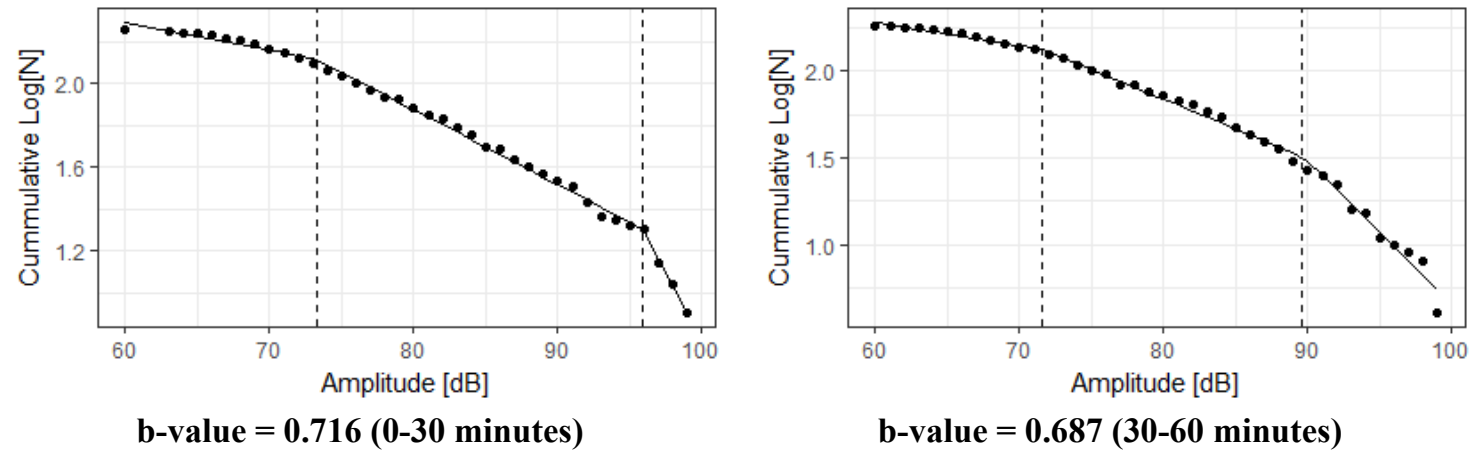

b-value $=0.687(30-60$ minutes $)$ 

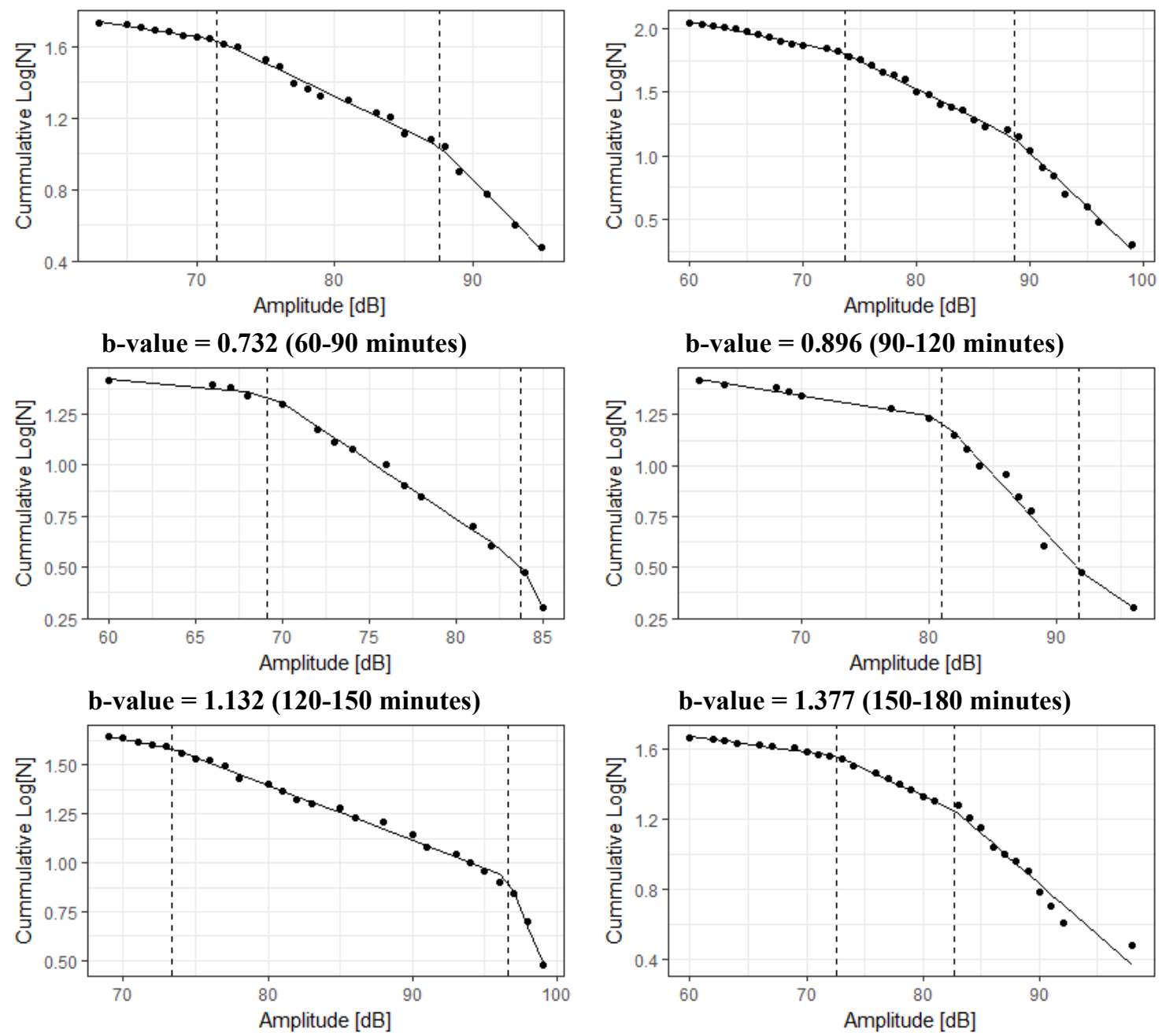

b-value $=0.567(180-210$ minutes $)$

b-value $=0.610(210-240$ minutes $)$

Figure 6.9. Transport test TT1: b-value analyses in 30-minute time interval plots

These b-values are plotted versus time in Figure 6.10 and show a steep drop in bvalue after hour three and could indicate the possibility of damage. Additional comparative data sets would be required to verify the occurrence of damage. 


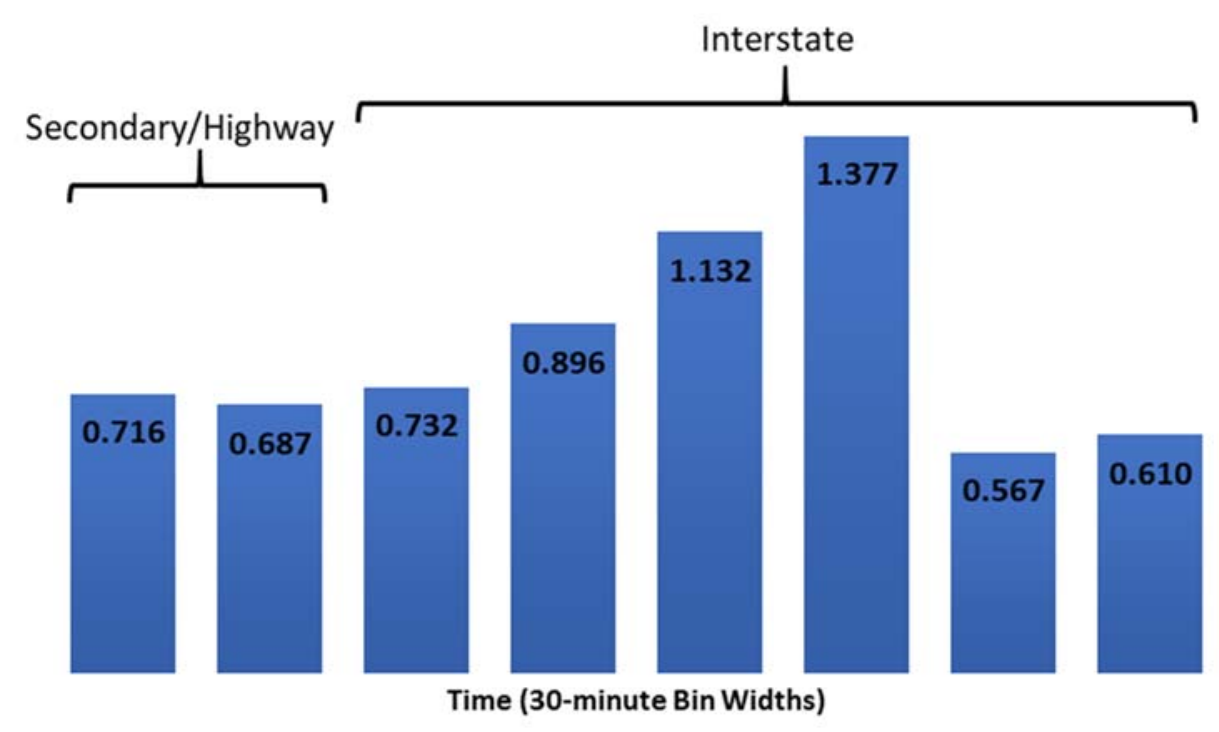

Figure 6.10. Transport test TT1: b-values in 30-minute time intervals

\subsubsection{Data Collection Process Observations}

The AE event data collected during TT1 had sparse and diffuse distributions of locations spaced on the vertical web. Observations by the data acquisition operator riding in the chase vehicle noted that the recorded $\mathrm{AE}$ events occurred mostly during vehicle maneuvers that tended to flex the girder, such as when cresting a hill, bottoming in a valley, and during tight turns or turns with abrupt movements. AE events did not appear to occur when hitting a pothole in the road or driving over a rumble strip. This suggests that the recorded $\mathrm{AE}$ events might be largely due to a release of internal energy and not from external energy injected into the girder by roadway conditions.

Finite element modeling of the stresses in the girder was conducted for insight into the source of these recorded AE events. The modeling used the ANSYS R18.2 academic version. The finite element model (FEM) was a quasi-static model and used quarter-beam symmetry to reduce the number of nodes and elements and associated computational effort. The material properties were standard values for concrete properties in the ANSYS 
database. Application of an equivalent shearing force accounted for the pre-stressing load from the pre-tensioning strands. Additionally, to model for bouncing and deflections of the beam during transport, the model was assessed with a gravity load of $1 \mathrm{~g}$ down and $1 \mathrm{~g}$ up. The finite element models in Figure 6.11 and Figure 6.12 show a relatively even stress distribution at the end zone which corresponds to the relatively evenly dispersed AE events recorded at the end zone during transport.

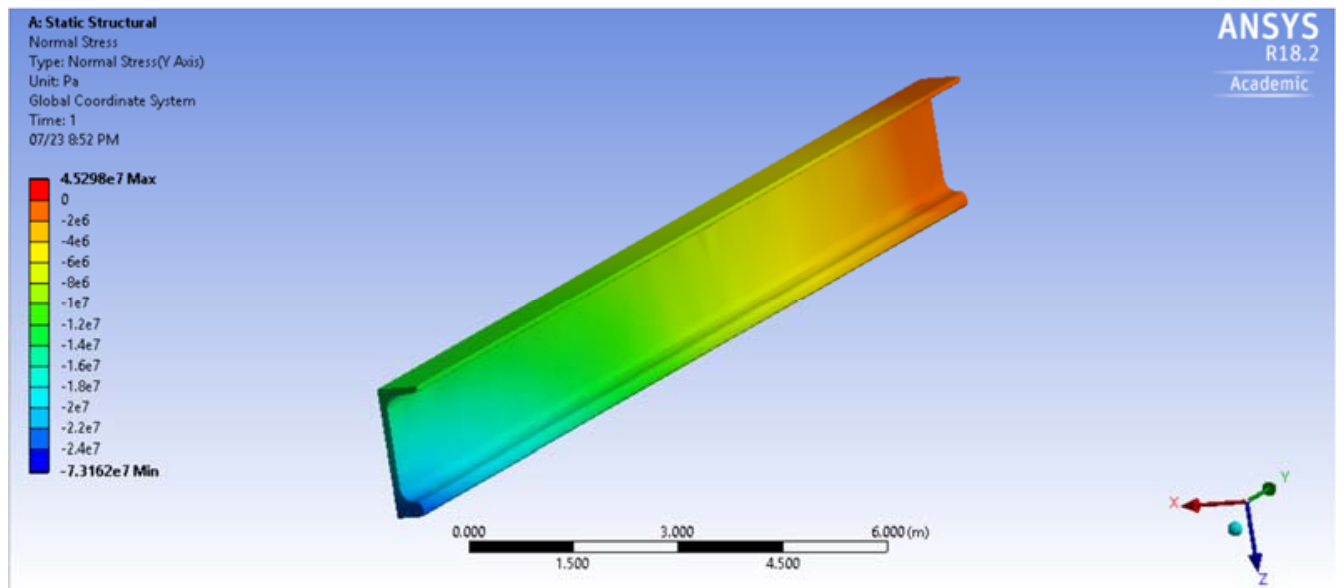

Figure 6.11. FEM quarter straight NEBT stress distribution with $1 \mathrm{~g}$ down, left side corresponds to mid-span and right side corresponds to the end zone

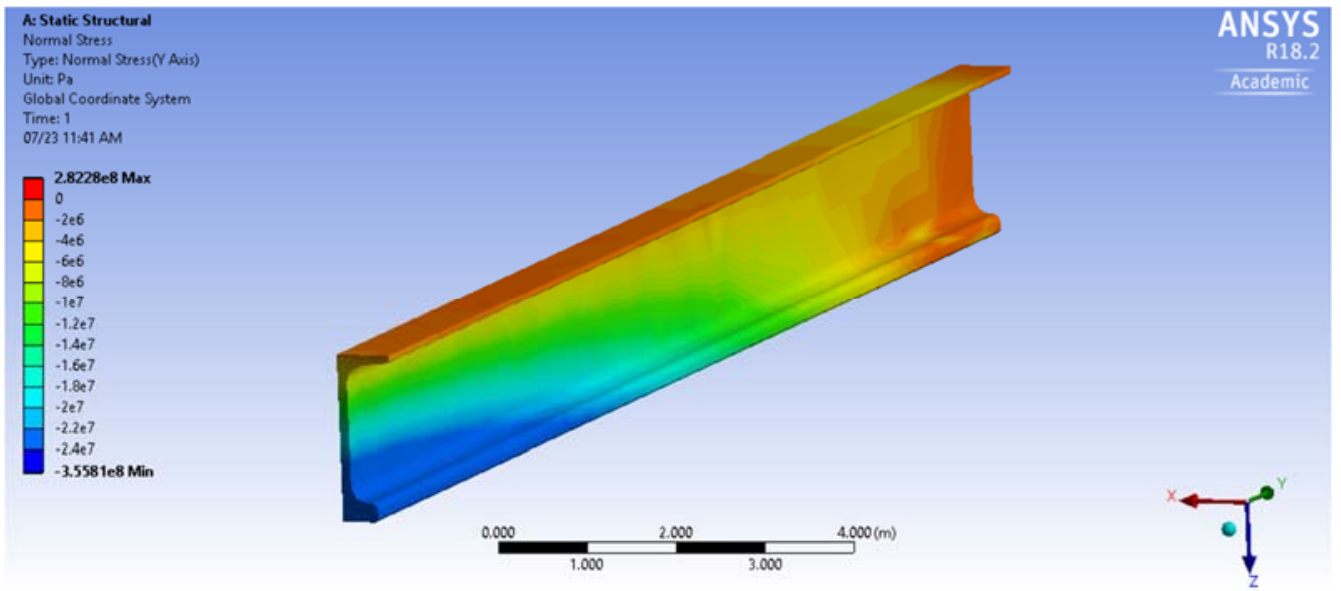

Figure 6.12. FEM quarter straight NEBT stress distribution with $1 \mathrm{~g}$ up, left side corresponds to midspan and right side corresponds to the end zone 


\subsection{Straight NEBT Mid-Span Data Collection (Transport Test 2)}

This was the second transport test (TT2) on June 25, 2018. The girder was a straight NEBT. The transport route was the same as shown in Figure 6.4.

\subsubsection{Equipment Layout}

The sensor arrangement placed eight $\mathrm{AE}$ transducers in a 2-D triangular grid array in the mid-span of the vertical web section of the NEBT girder. All other aspects of testing were the same as with test TT1.

\subsubsection{Representative Data Collection}

The sensor configuration of the second transport test was a 2-D planar triangular grid array placed mid-span of the straight section NEBT, Figure 6.13. During monitoring of the middle region of a straight NEBT over the course of roughly 160 miles (258 kilometers) in transport from the fabrication location to the installation location. The number of recorded AE events totaled 2,628. The recorded AE events tended to occur in the upper half of the web section of the modeled NEBT girder. Travel along a secondary highway (US 7) from Middlebury to Burlington produced events at a slightly higher rate than along the interstate (I-89 and I-91) from Burlington to Rockingham, Figure 6.13.d. Visual observation found no new cracks formed and the existing cracks did not grow during transport. 


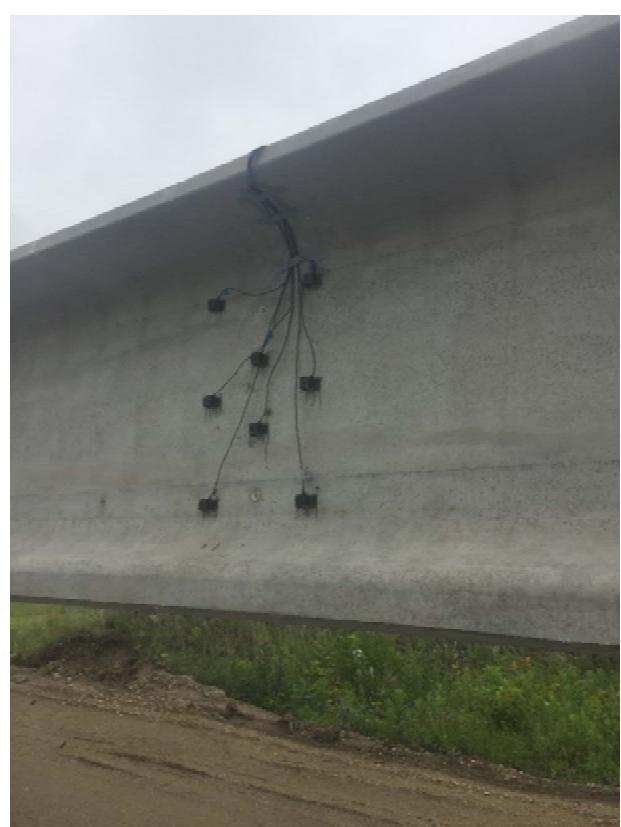

(a)

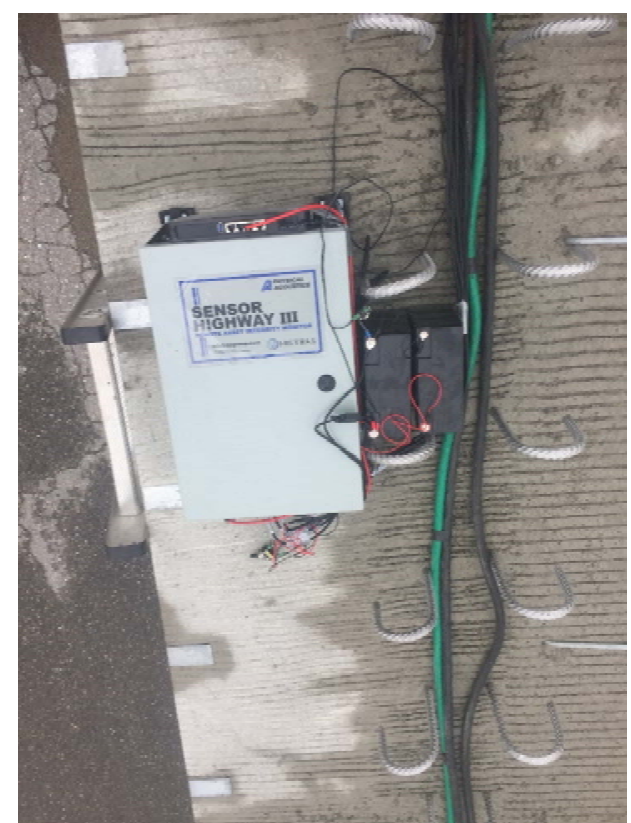

(b)

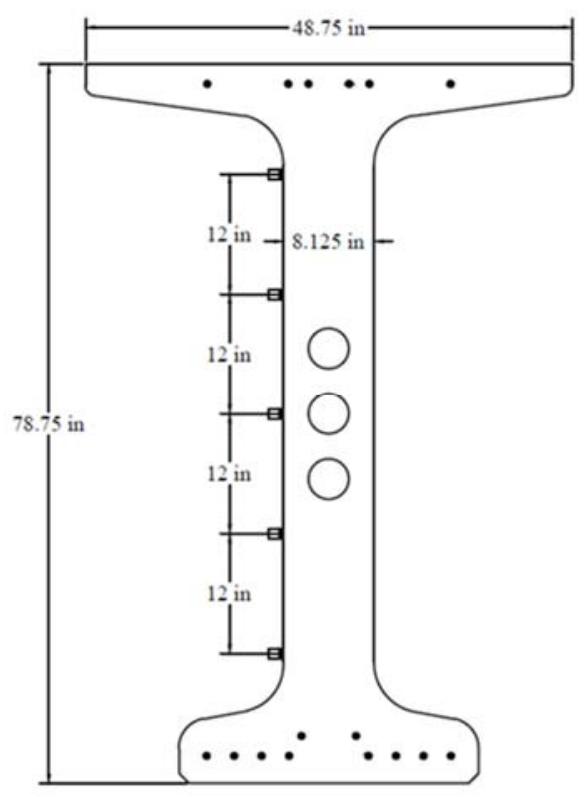

(c)

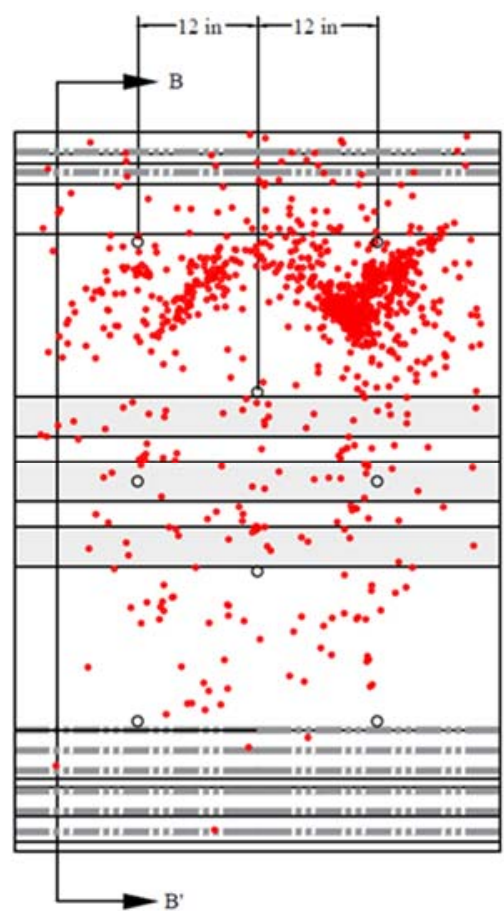

(d)

Figure 6.13. Transport Test 2 - AE measurements of the mid-span vertical web of a straight NEBT during transport with 2,628 recorded $\mathrm{AE}$ events. (a) photograph of 2-D sensor array, (b) photograph of equipment set-up, (c) cross-section of NEBT tested, (d) profile view of NEBT including AE event locations, sensors, and observed cracks 
The recorded AE events depicted in Figure 6.13 were also plotted by amplitude as shown in Figure 6.14. The amplitude plot of AE events shows a clustering of mediumhigh amplitude AE events (yellow: $80 \mathrm{~dB}-89 \mathrm{~dB}$ ) in the upper right portion of the NEBT girder with a high concentration of low amplitude (blue: $60 \mathrm{~dB}-69 \mathrm{~dB}$ ) and medium-low amplitude AE events (green: $70 \mathrm{~dB}-79 \mathrm{~dB}$ ). AE events are concentrated in the upper third of the NEBT girder. High amplitude AE events (red: $90 \mathrm{~dB}-99 \mathrm{~dB}$ ) are limited and are diffuse.
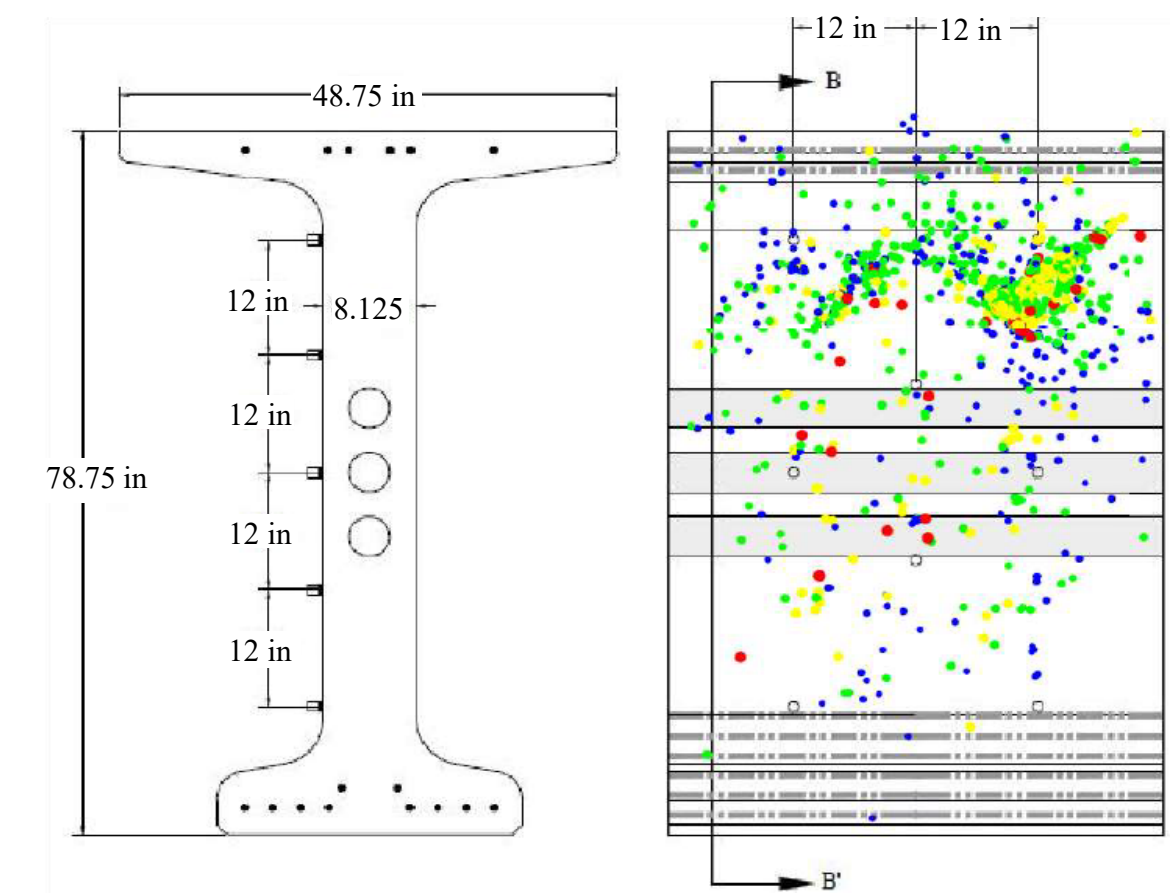

Blue: 60dB - 69dB Green: 70dB - 79dB Yellow: 80dB - 89dB Red: 90dB - 100dB

Figure 6.14. Transport Test 2 - AE measurements plotted by amplitude of the mid-span vertical web of a straight NEBT during transport with 2,628 recorded AE events.

Recorded amplitude distributions from the mid-span of a straight NEBT during transport using a 2-D sensor array indicate a semi-normal distribution between the threshold lower limit (60 dB) and upper limit (99 dB) (Figure 6.15). 


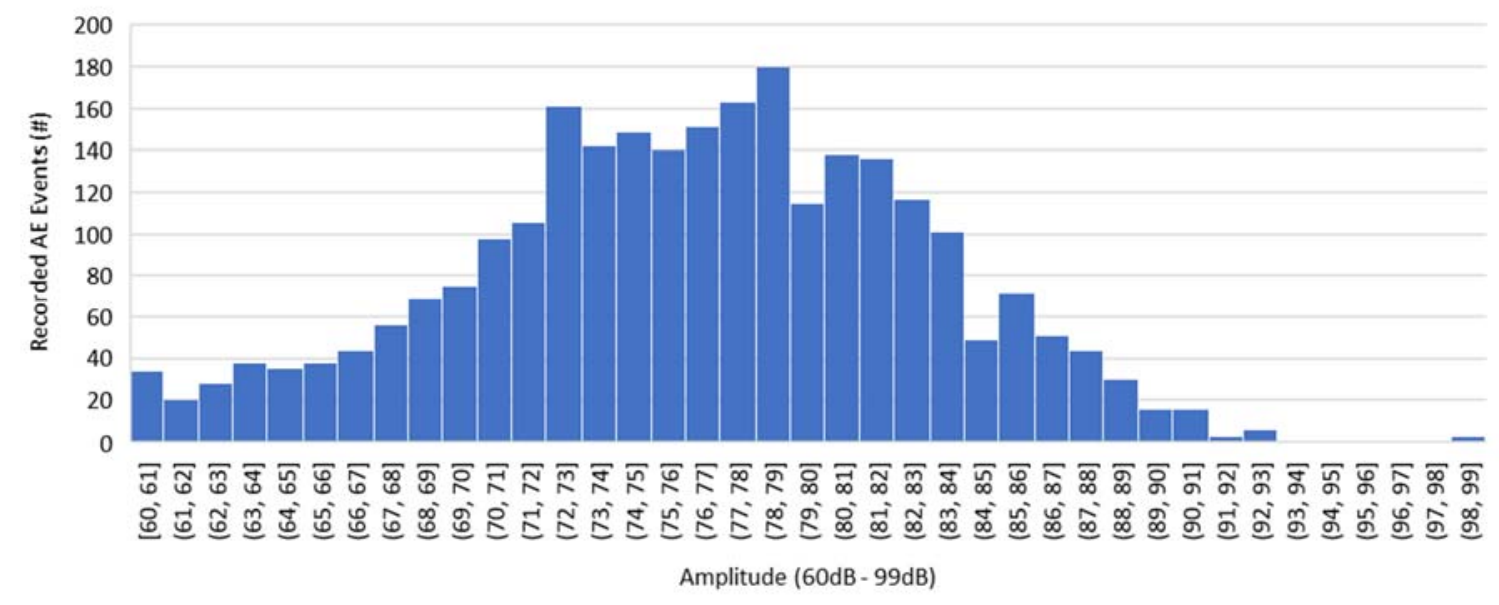

Figure 6.15. Amplitude distribution for a 2D sensor array transport test of the middle zone of a straight section NEBT girder with 2,628 recorded events

The AE event time histories collected during TT2 and plotted in Figure 6.16, indicate a greater level of $\mathrm{AE}$ event occurrences when on secondary/highway roadways compared to AE event data collection on the interstate. Additionally, no AE events were collected during stops. Although there still is a decreasing trend in the number of $\mathrm{AE}$ events with time; this decreasing trend is less distinct than that identified in TT1.

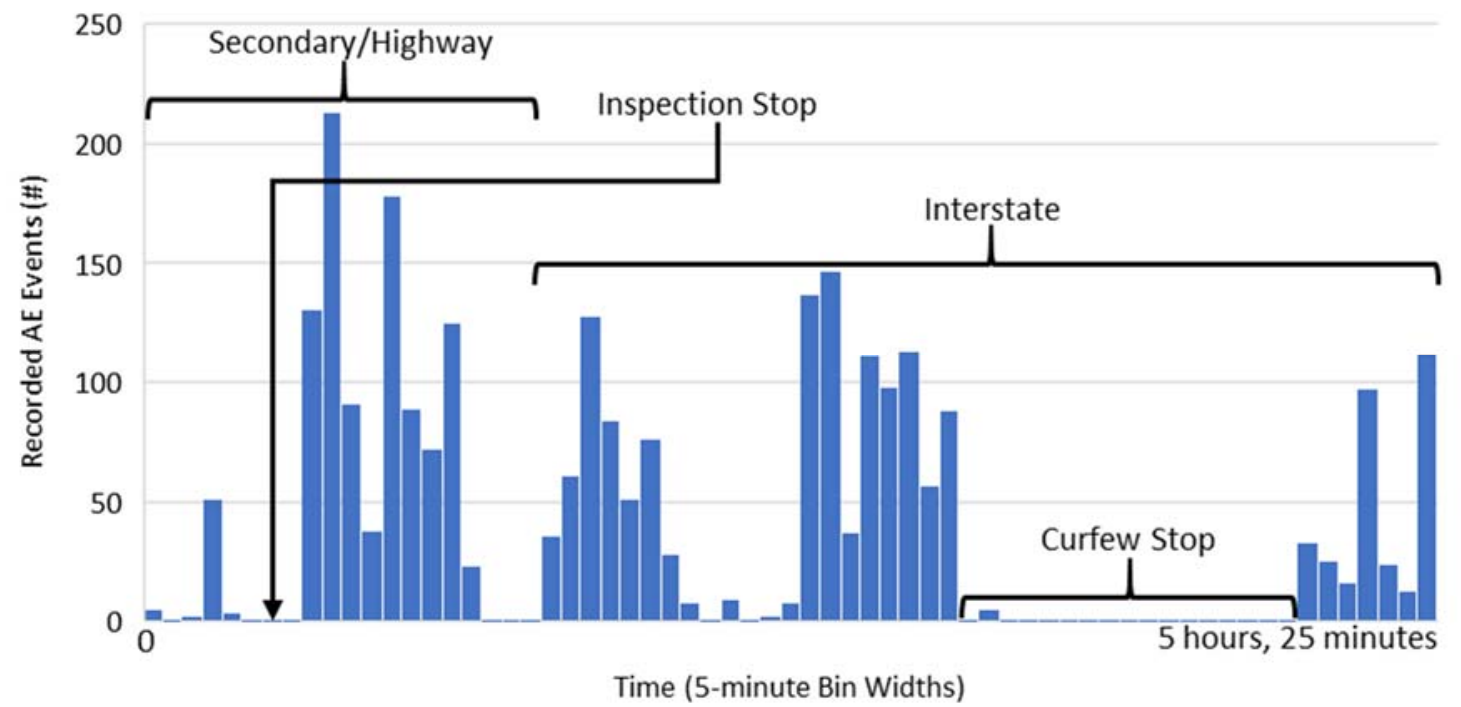

Figure 6.16. AE event density during transport testing of middle zone region of straight NEBT, with a total event count of 2,628 
The b-value regression plots in 30-minute increments of time during TT2 is shown in Figure 6.17.
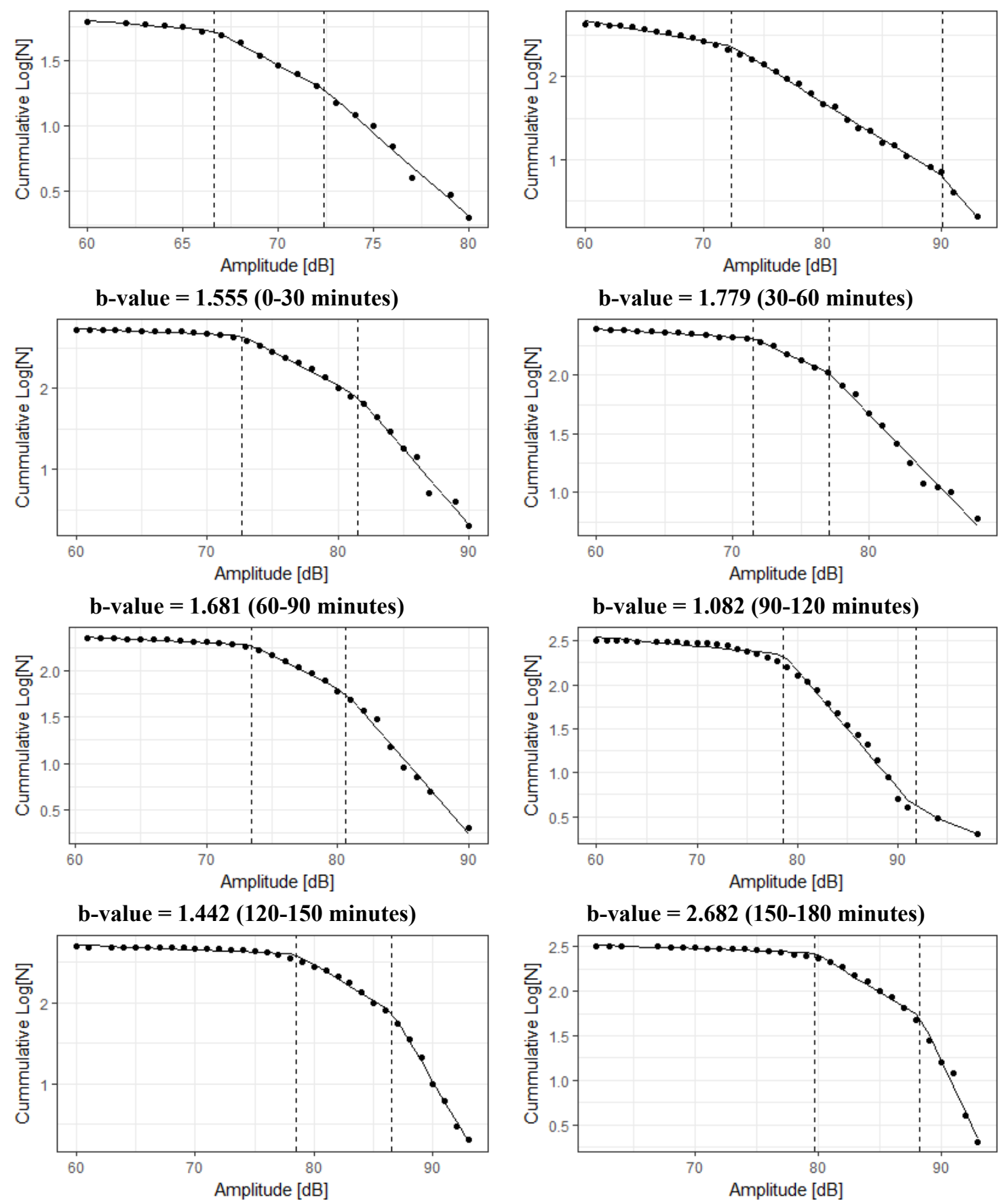

b-value $=1.775(180-210$ minutes $)$

b-value $=1.673$ (300-330 minutes)

Figure 6.17. Transport test TT2: b-value analyses in 30-minute time interval plots 
These b-values are plotted versus time in Figure 6.18 and show a relatively uniform b-value distribution with exception to the fourth and sixth 30-minute intervals which indicate a drop and spike in b-value, respectively. Additional comparative data sets would be required to verify the occurrence of damage.

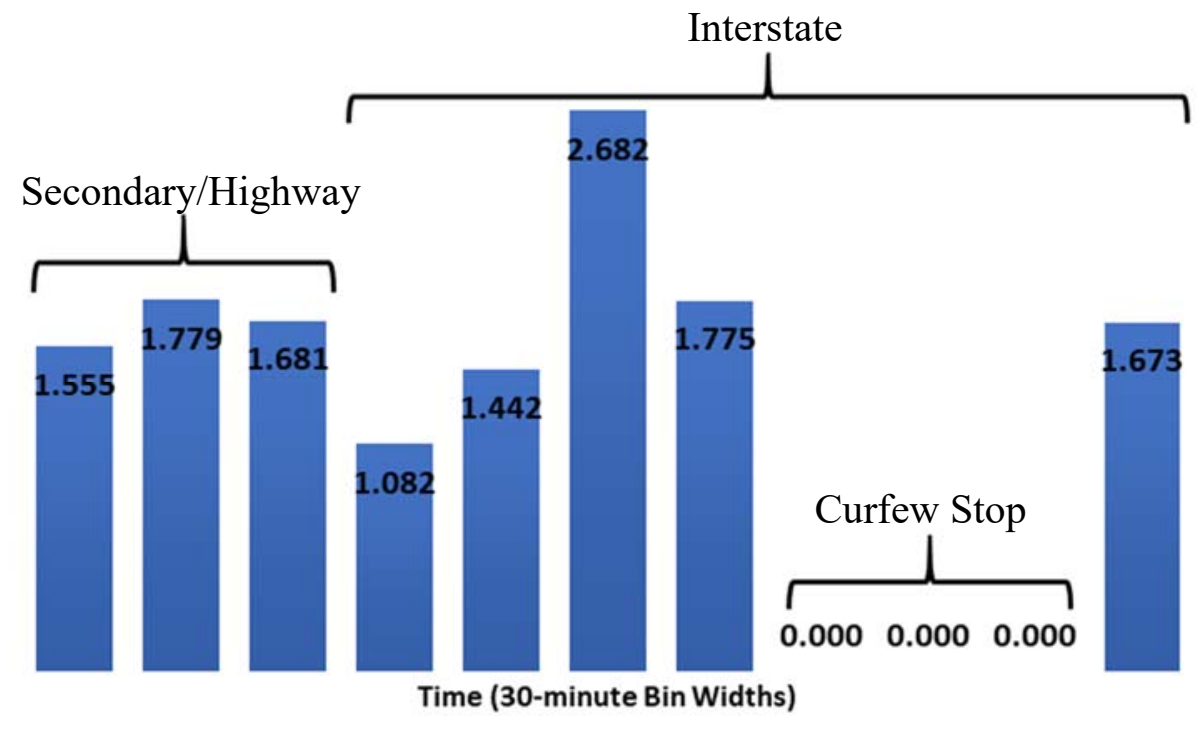

Figure 6.18. Transport test TT2: b-values in 30-minute time intervals

\subsubsection{Data Collection Process Observations}

AE field transport data collected from the middle zone of a straight NEBT section were dense and concentrated in the upper region of the beam. The events occurred mostly during flexure-inducing vehicle maneuvers, such as when cresting a hill, bottoming in a valley, and during tight turns or turns with abrupt movements. Again, hitting a pothole in the road or driving over a rumble strip did not induce many AE events. This suggests that the recorded AE events may be due to internal energy release and not from the external energy from roadway conditions.

A finite element model using ANSYS R18.2 academic version calculated the stress distribution in the girder with the goal of determining if regions high stress correspond to 
the locations of AE events. The finite element model (FEM) was quasi-static and used quarter beam symmetry to reduce the number of nodes and elements and associated computational effort. The model used the same standard concrete material properties as for TT1 and approximated the action of the pre-stressing strands as a shearing force. Again, to model for bouncing and deflections of the beam during transport, the model was assessed with a gravity load of $1 \mathrm{~g}$ down and $1 \mathrm{~g}$ up. The finite element model results shown in Figure 6.19 and Figure 6.20 indicate stress distributions commensurate with the recorded AE event distributions. Further confirmation comes from an examination of the shear and moment diagram for the beam during transport and nominal in-service loading, Figures 6.21 and 6.22 . The FEM shows a compression at the bottom of the beam moving toward tension at the top of the beam which corresponds to the recorded AE events being in the upper regions on the middle zone of the straight NEBT during transport. This stress distribution with the top of the beam in tension and the bottom of the beam in compression is due to a vertically asymmetric distribution of pre-stressing strands with more strands in the bulb at the bottom.

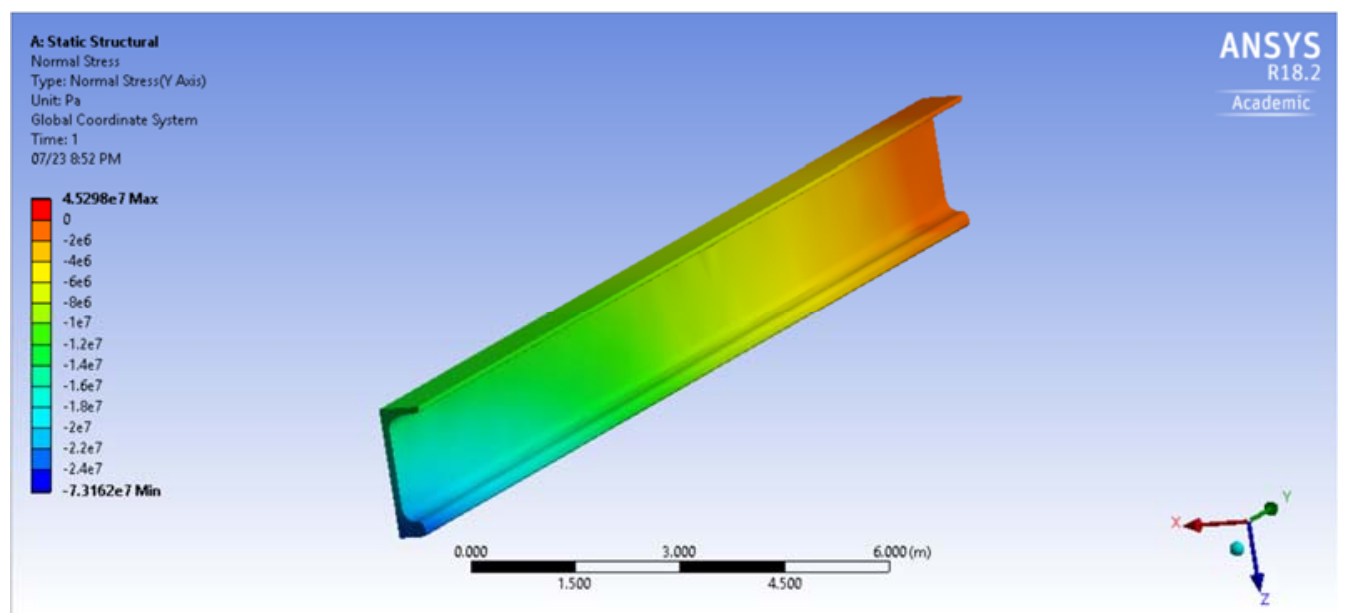

Figure 6.19. FEM quarter straight NEBT stress distribution with $1 \mathrm{~g}$ down, left side corresponds to mid-span and right side corresponds to the end zone 


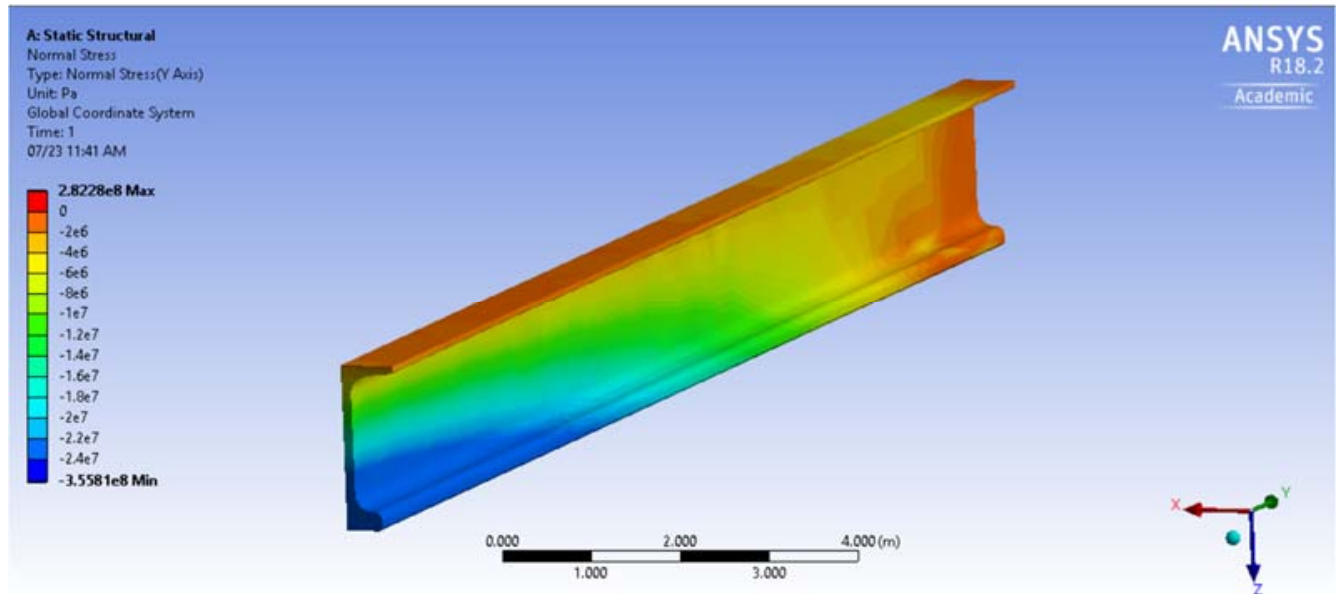

Figure 6.20. FEM quarter straight NEBT stress distribution with $1 \mathrm{~g}$ up, left side corresponds to midspan and right side corresponds to the end zone

The shear and bending moment diagrams of the straight span NEBT girders during transport as illustrated in Figure 6.21, are similar to the shear and bending moment diagrams of the straight span NEBT girders during in-service loading as illustrated in Figure 6.22. 

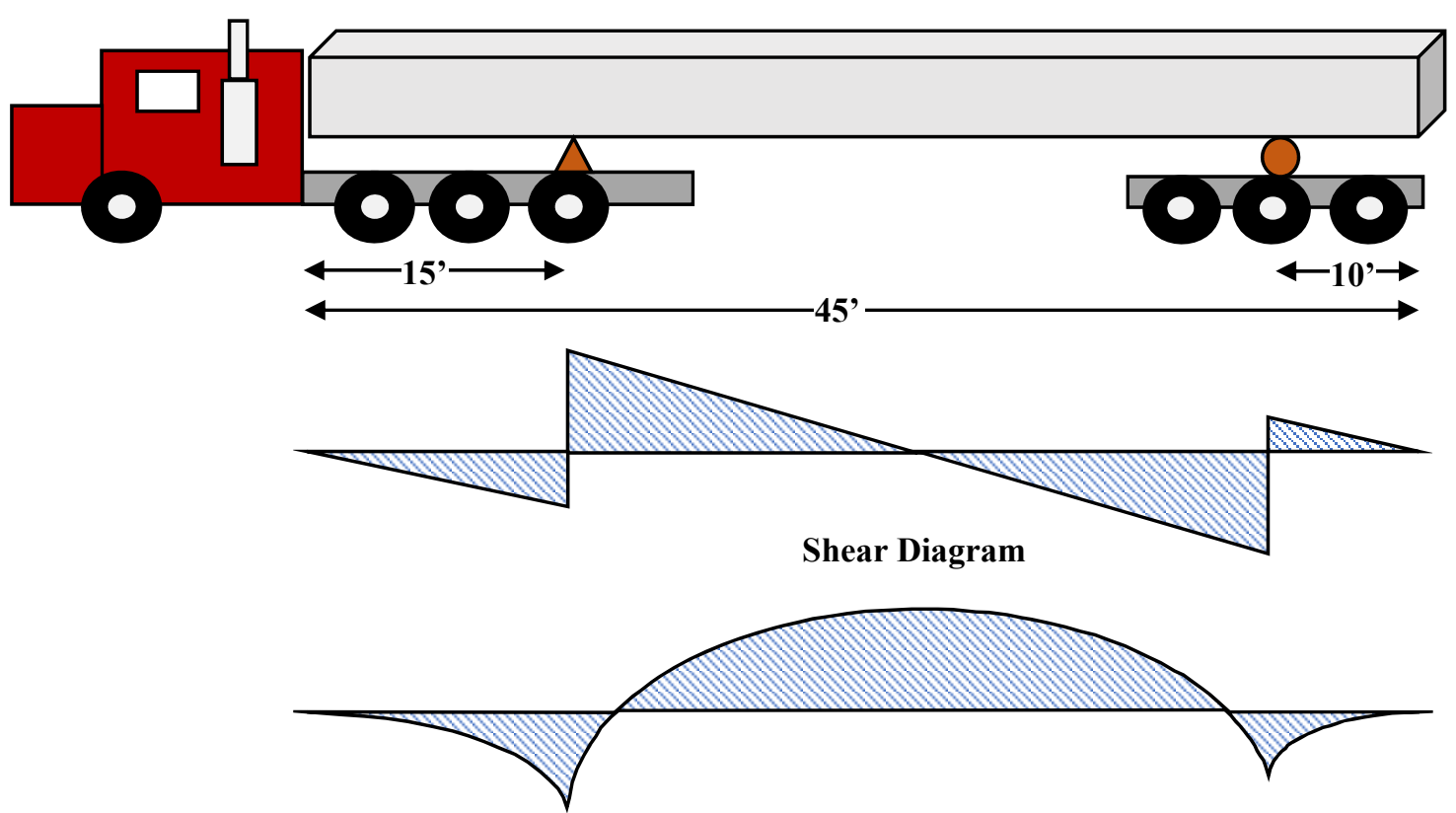

Bending Moment Diagram

Figure 6.21. Straight span NEBT shear and bending moment diagrams during transport

The locations that have an opposite loading between in-service loading and transport are at the end zones. This may be indicative of the end zone AE event patterns during transport.
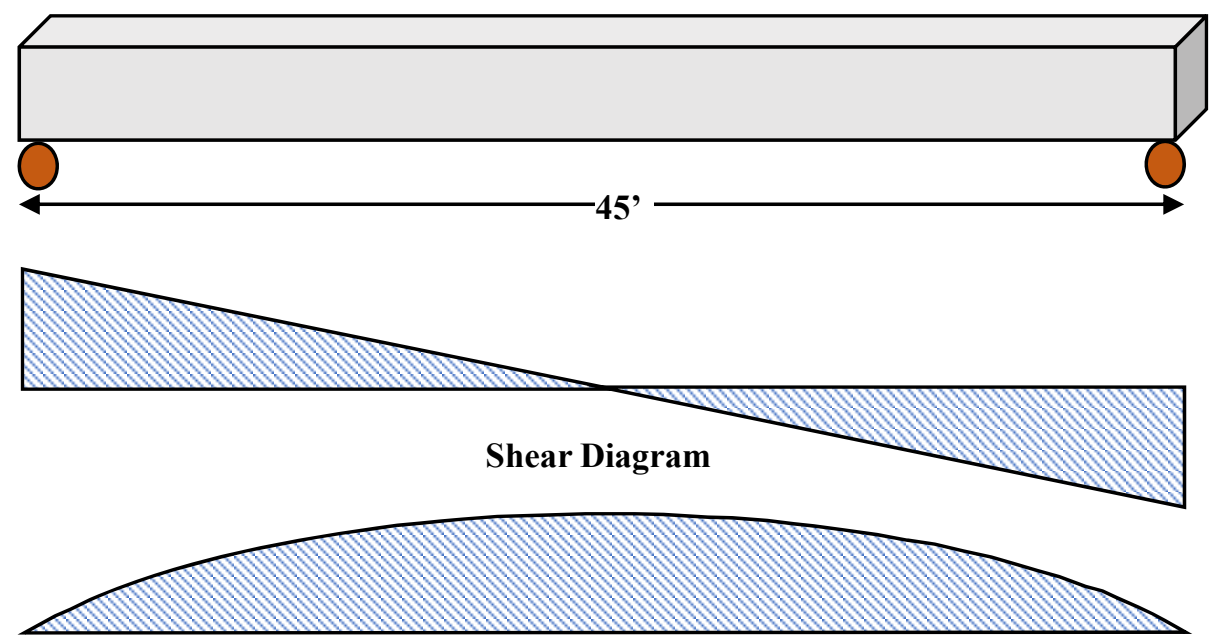

Bending Moment Diagram

Figure 6.22. Straight span NEBT shear and bending moment diagrams during in-service loading 


\subsection{Hammerhead NEBT Mid-Span Data Collection (Transport Test 3)}

This was the third transport test (TT3) on June 26, 2018. The girder was a hammerhead (haunch) NEBT. The AE data collection zone was the vertical web of the girder at midspan. The transport route was the same as shown in Figure 6.4.

\subsubsection{Equipment Layout}

The sensor arrangement placed eight AE transducers in a 2-D array in the mid-span of the vertical web section of the NEBT girder. All other aspects of testing were the same as with test TT1.

\subsubsection{Representative Data Collection}

The sensing configuration for the third transport test used a 2-D planar triangular sensor array placed on the vertical web of the mid-span of a hammerhead NEBT girder (Figure 6.23). During transport, the truck supports the girder at both ends, approximately in a simple support configuration. Following installation on the bridge, the support conditions change to a fixed cantilever support mid-span on a column and free at the ends.

The monitoring of the middle region of a hammerhead NEBT ran over the course of roughly 160 miles (258 kilometers) in transport from the fabrication location to the installation location. The number of recorded $\mathrm{AE}$ events totaled 91,723. The recorded $\mathrm{AE}$ events tended to occur in the lower two thirds of the web section of the NEBT modeled. Travel along a secondary highway (US 7) from Middlebury to Burlington produced events at a slightly higher rate than along the interstate (I-89 and I-91) from Burlington to Rockingham, Figure $6.23 \mathrm{c}$. A visual observation of the girder found no new cracks or growth of existing cracks during transport. 


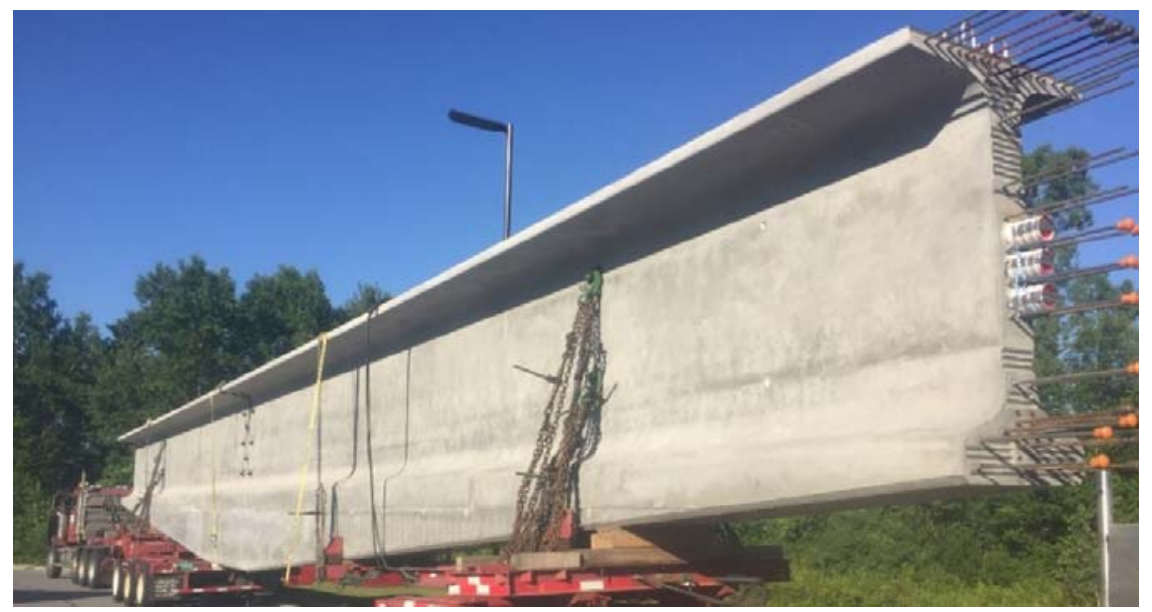

(a)

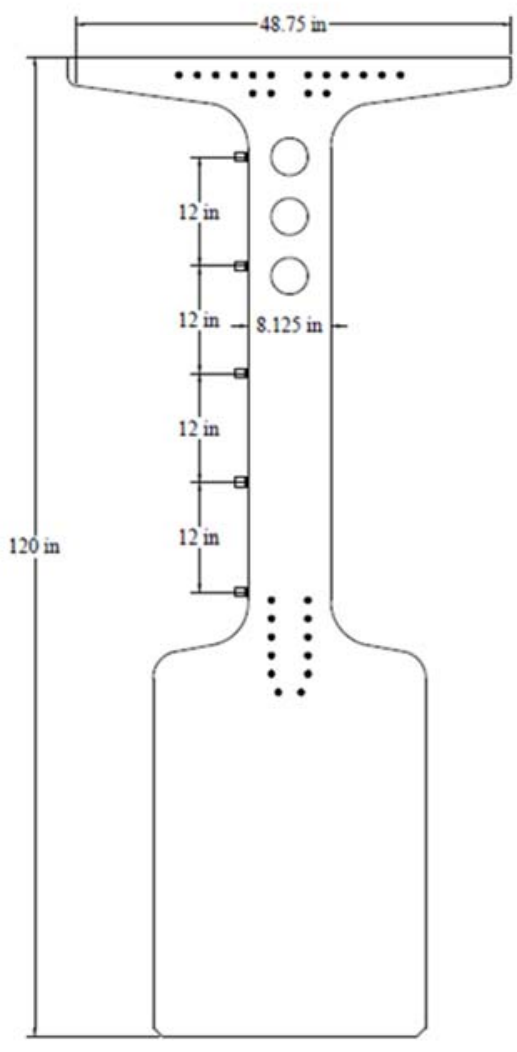

(b)

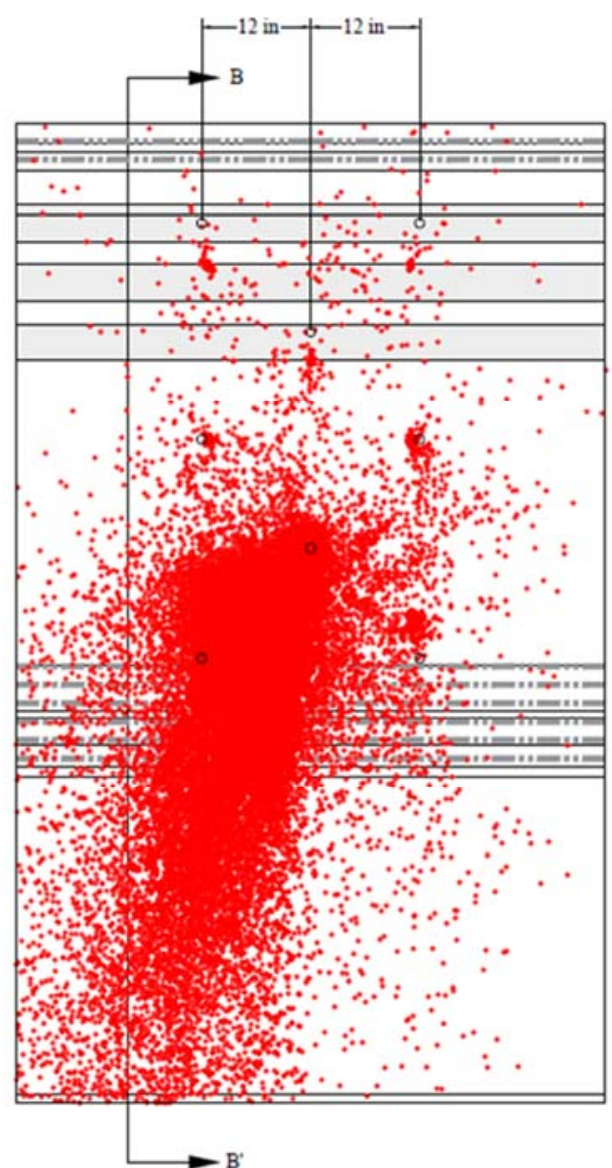

(c)

Figure 6.23. Transport Test 3 - AE measurements of the mid-span vertical web of a hammerhead NEBT during transport with 91,723 recorded AE events. (a) photograph of girder on truck with 2-D sensor array and equipment set-up, (b) mid-span cross-section of tested NEBT, (c) profile view of NEBT including AE event locations and sensors. Note that the post-tensioning strand ducts are at a higher location in the mid-span cross sections of (b) and (c) than at the end zone as shown emerging from the end of the section face in (a). 
The recorded AE events are depicted in Figure 6.23 were also plotted by amplitude as shown in Figure 6.24. The amplitude plot of AE events show a high concentration of low amplitude (blue: $60 \mathrm{~dB}-69 \mathrm{~dB}$ ), medium-low amplitude (green: $70 \mathrm{~dB}-79 \mathrm{~dB}$ ), and medium-high amplitude AE events (yellow: $80 \mathrm{~dB}-89 \mathrm{~dB}$ ) AE events in the "belly" of the NEBT girder with high amplitude (red: $90 \mathrm{~dB}-99 \mathrm{~dB}$ ) AE events being limited in quantity and diffuse.
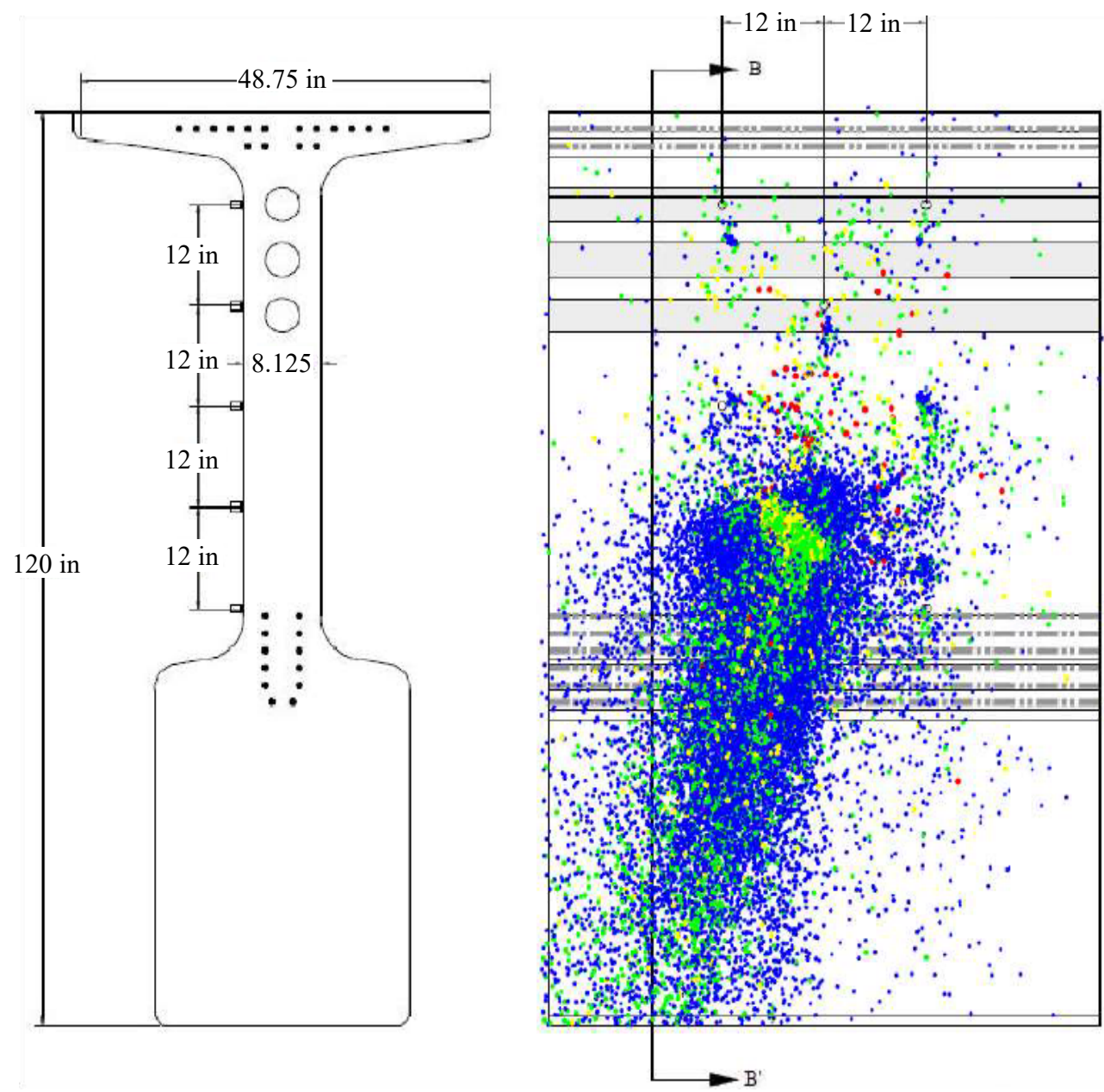

Blue: 60dB - 69dB Green: 70dB - 79dB Yellow: 80dB - 89dB Red: 90dB - 100dB

Figure 6.24. Transport Test 3 - AE measurements plotted by amplitude of the end zone vertical web of a straight NEBT during transport with 91,723 recorded $A E$ events. 
Recorded amplitude distributions from the mid-span of the hammerhead NEBT during transport using a 2-D sensor array show the end of a normal distribution (Figure 6.25). The distribution appears to be cut short in the lower amplitudes which may indicate the amplitude threshold should be lowered for future tests of the middle zone of a hammerhead NEBT.

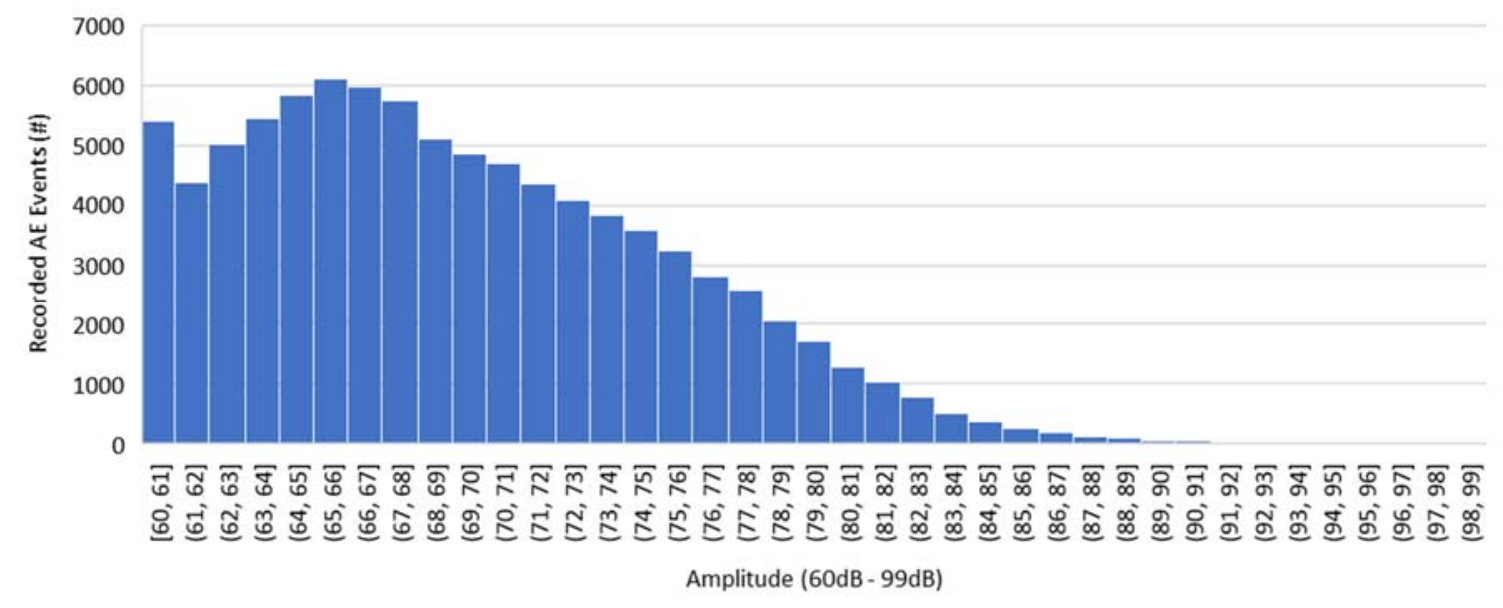

Figure 6.25. Amplitude distribution for a 2D sensor array transport test of the middle zone of a hammerhead section NEBT girder with 91,723 recorded events

The AE event time histories collected during TT3 and plotted in Figure 6.26, indicate a greater level of $\mathrm{AE}$ event occurrences when on interstate compared to $\mathrm{AE}$ event data collection on the secondary/highway roadways. This is the opposite of what was observed during TT1 and TT2. This could be due to the difference in geometries, stress distributions, and loading between the straight NEBT sections and hammerhead NEBT sections. Additionally, no AE events were collected during stops. 


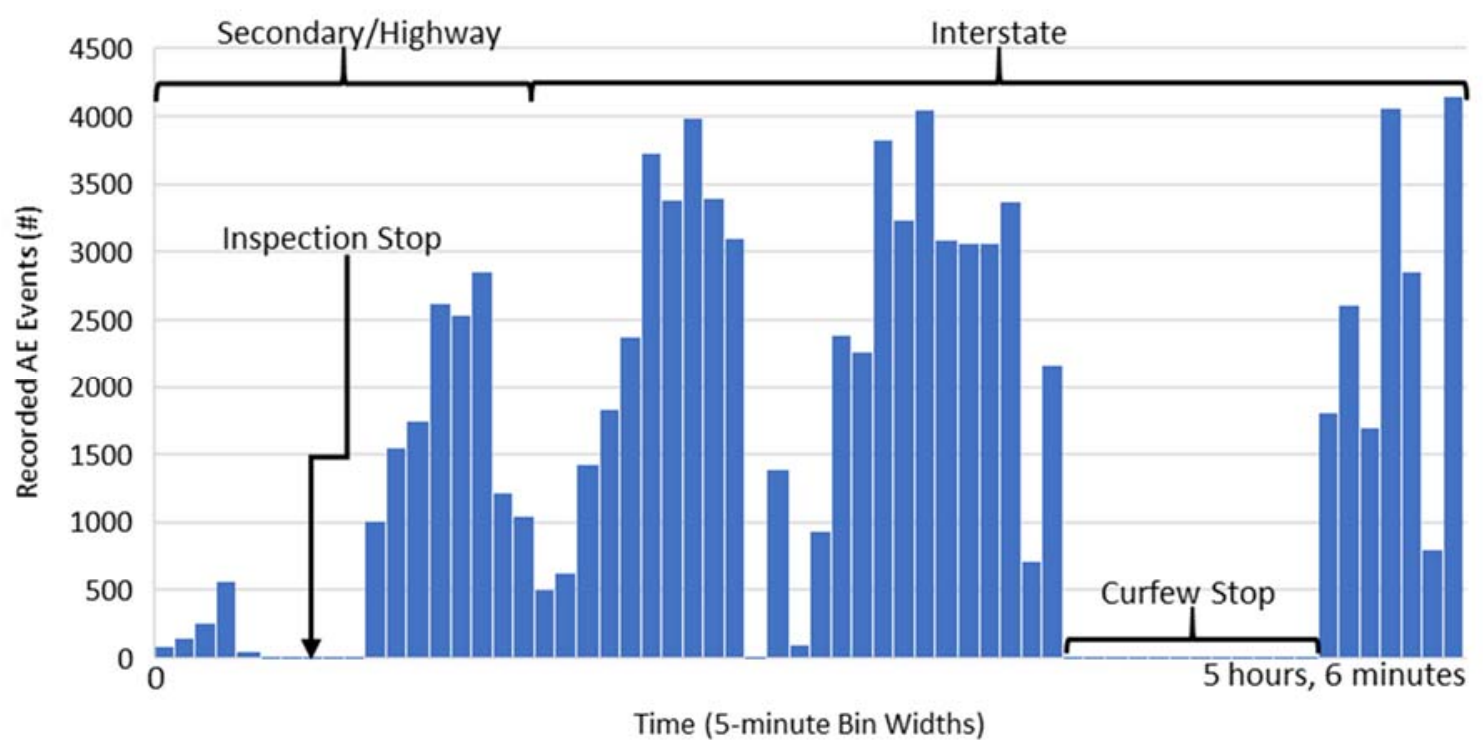

Figure 6.26. AE event density during transport testing of middle zone region of hammerhead NEBT, with a total event count of 91,723

The b-value regression plots in 30-minute increments of time during TT3 is shown in Figure 6.27.
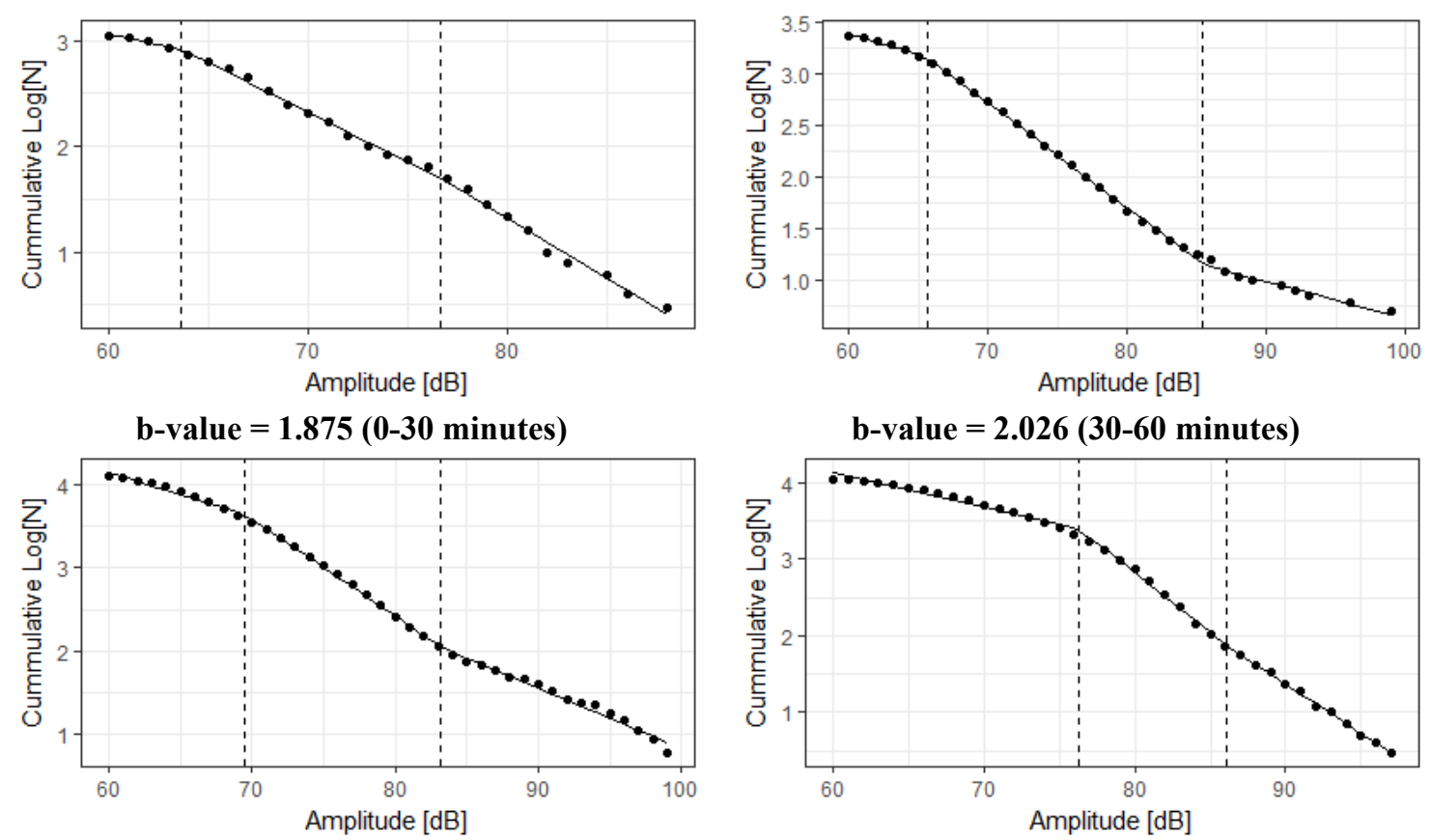

b-value $=2.322(60-90$ minutes $)$

b-value $=3.111(90-120$ minutes $)$ 

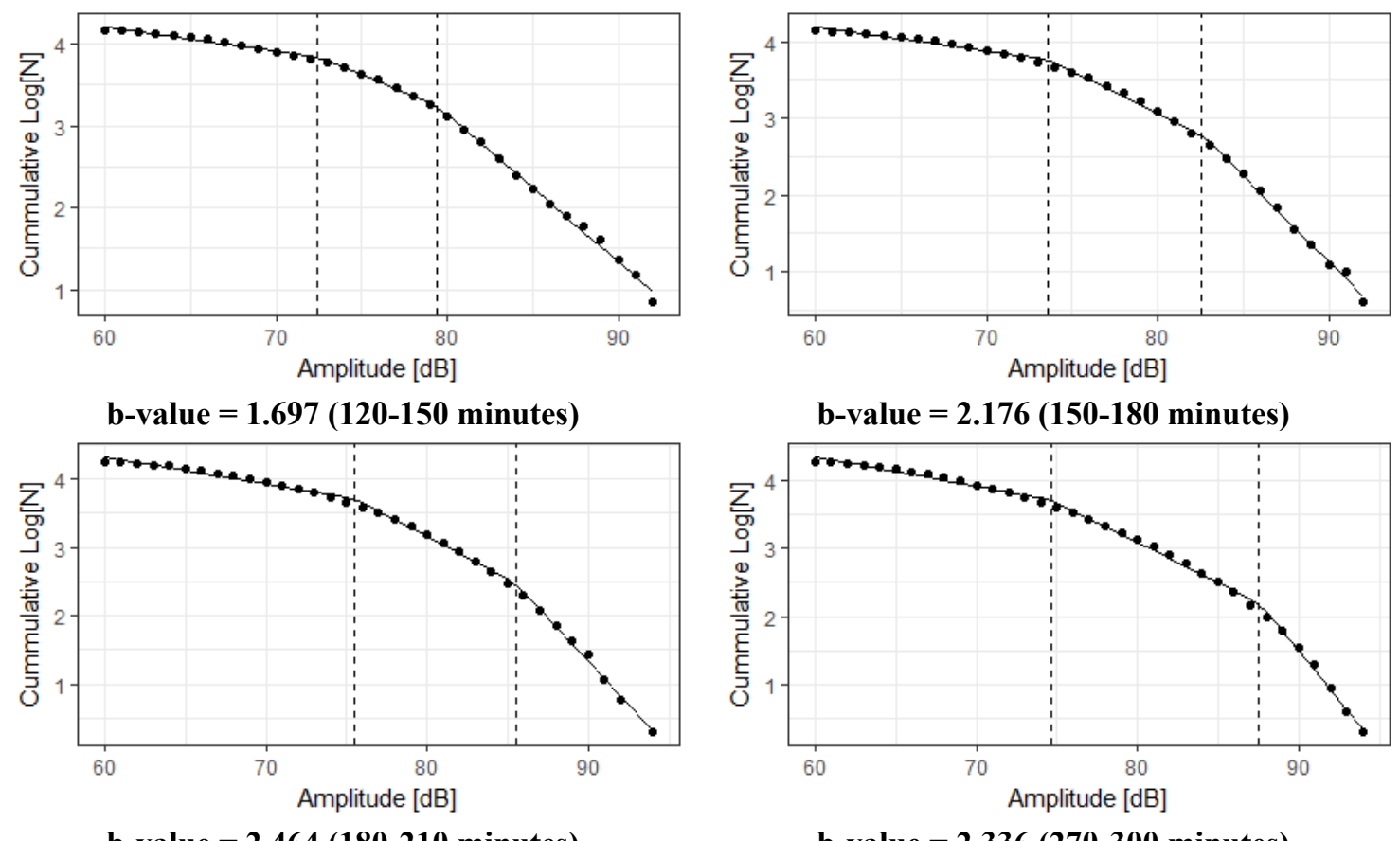

b-value $=2.464$ (180-210 minutes)

b-value $=2.336(270-300$ minutes $)$

Figure 6.27. Transport test TT3: b-value analyses in 30-minute time interval plots

These b-values are plotted versus time in Figure 6.28 has an initial steady increase in b-value, followed by a steep drop after the first 30-minute interval on the instate and then a slight increasing trend. Additional comparative data sets would be required to verify the occurrence of damage.

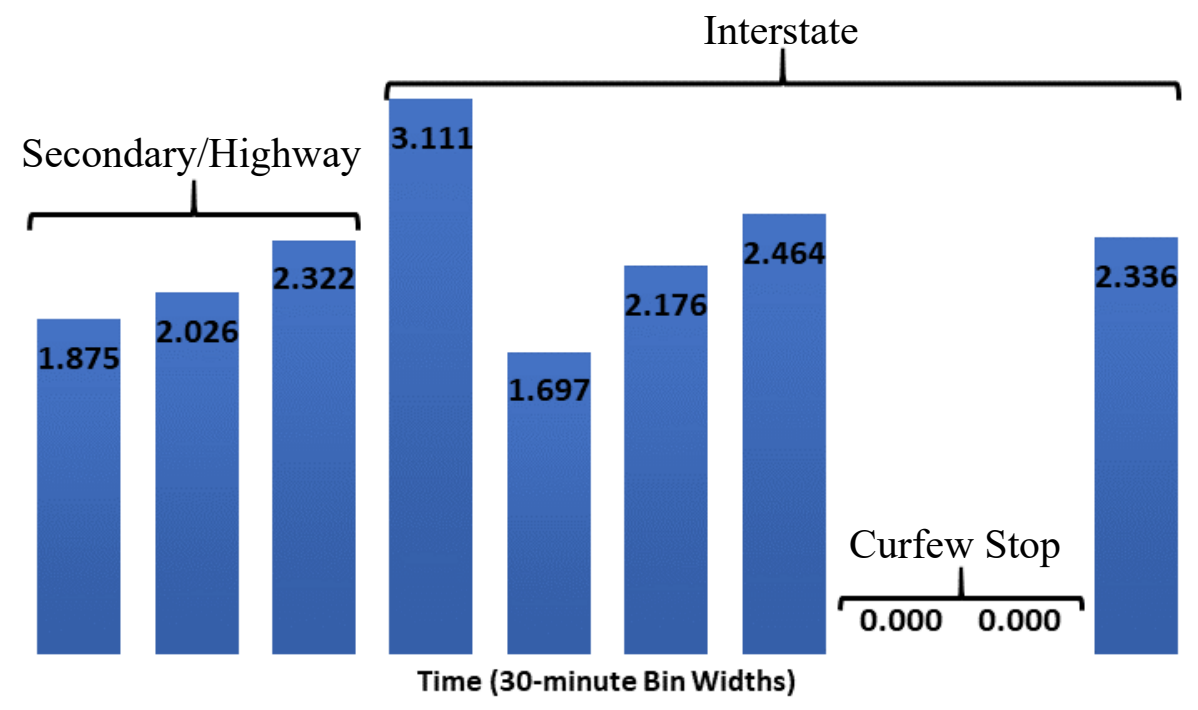

Figure 6.28. Transport test TT3: b-values in 30-minute time intervals 


\subsubsection{Data Collection Process Observations}

AE field transport data were collected from the middle zone of a hammerhead NEBT. AE event data collected from the middle zone of the hammer head NEBT were abundant and concentrated in the low region or "belly" of the beam. It was noted during transport that the occurrence of AE events was similar to that of TT1 and TT2. The events occurred mostly during flexure-inducing vehicle maneuvers, such as when cresting a hill, bottoming in a valley, and during tight turns or turns with abrupt movements. Similar to TT1 and TT2, hitting a pothole in the road or driving over a rumble strip did not induce many AE events. This suggests that the recorded AE events may be due to internal energy release and not from external energy from roadway conditions.

Similar to TT1 and TT2, a finite element model using ANSYS R18.2 academic version calculated the stress distribution in the girder with the goal of determining if regions high stress correspond to the locations of $\mathrm{AE}$ events was built. The finite element models shown in Figures 6.29 and 6.30 indicate stress distributions commensurate with the recorded AE event distributions. Further confirmation comes from an examination of the shear and moment diagrams, Figures 6.31 and 6.32. The FEM shows a compression at the top of the beam moving toward tension at the bottom of the beam which corresponds to the recorded $\mathrm{AE}$ events being in the lower regions on the middle zone of the hammerhead NEBT during transport. This stress distribution with the top of the beam in compression and the bottom of the beam in tension is due to the mass of the "belly" of the hammerhead NEBT overpowering the pre-stress that would otherwise make the beam camber. This reflects the design of the hammerhead NEBT as it will sit on a pier cap supported at the 
middle, essentially reversing the moment distribution. Only during craning and transport does the hammerhead NEBT experience this type of loading and stress distribution.

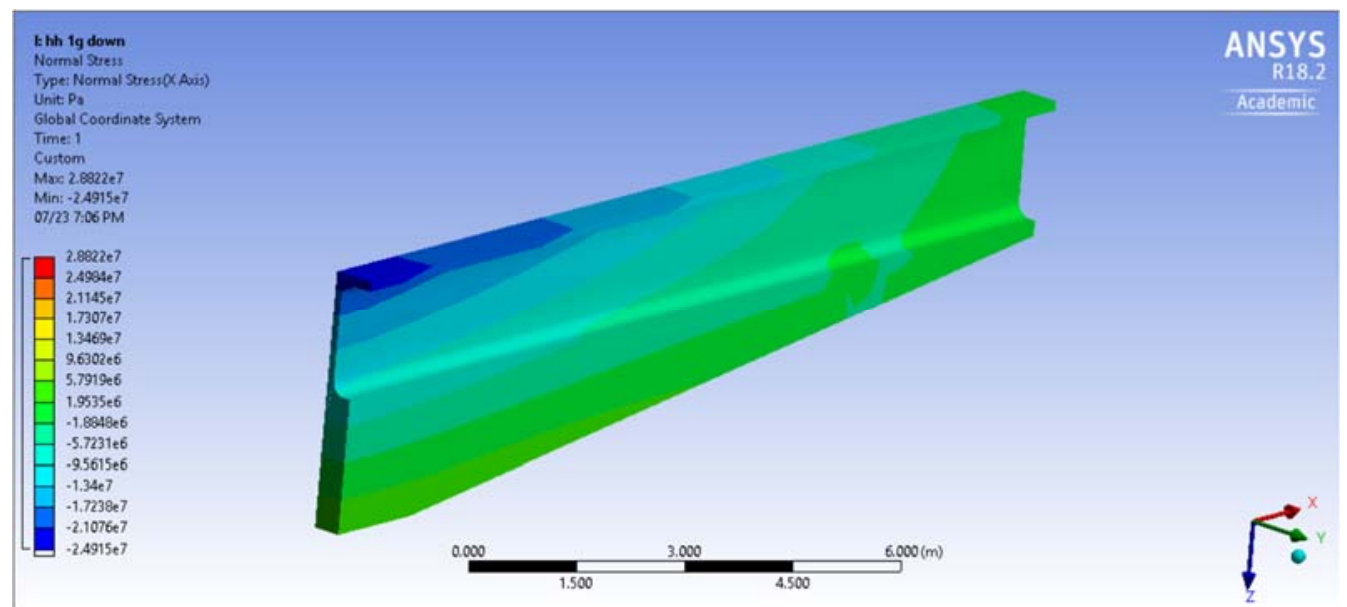

Figure 6.29. FEM quarter hammerhead NEBT stress distribution with $1 \mathrm{~g}$ down, left side corresponds to mid-span and right side corresponds to the end zone

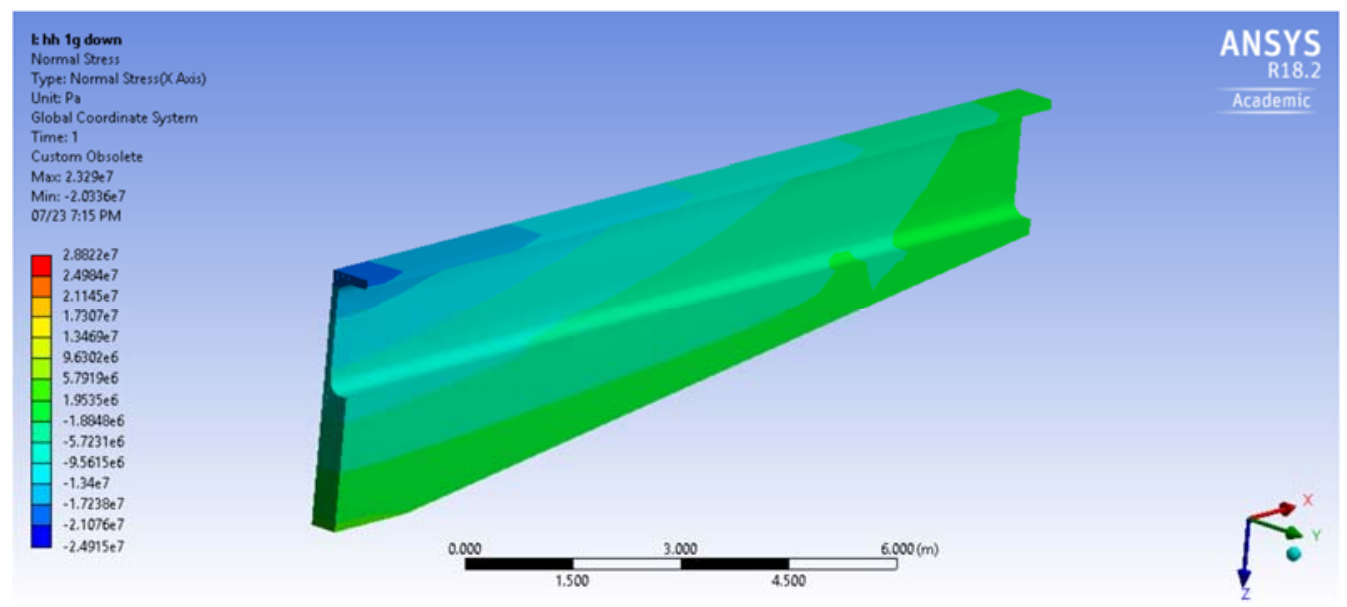

Figure 6.30. FEM quarter hammerhead NEBT stress distribution with $1 \mathrm{~g}$ up, left side corresponds to mid-span and right side corresponds to the end zone

The shear and bending moment diagrams of the hammerhead span NEBT girders during transport as illustrated in Figure 6.31, are the opposite to the shear and bending moment diagrams of the hammerhead span NEBT girders during in-service loading as illustrated in Figure 6.32. This makes the transport process for the hammerhead span NEBT girders of particular interest because in this process these hammerhead NEBT girders experience a unique stress pattern that is the opposite of the intended design. 


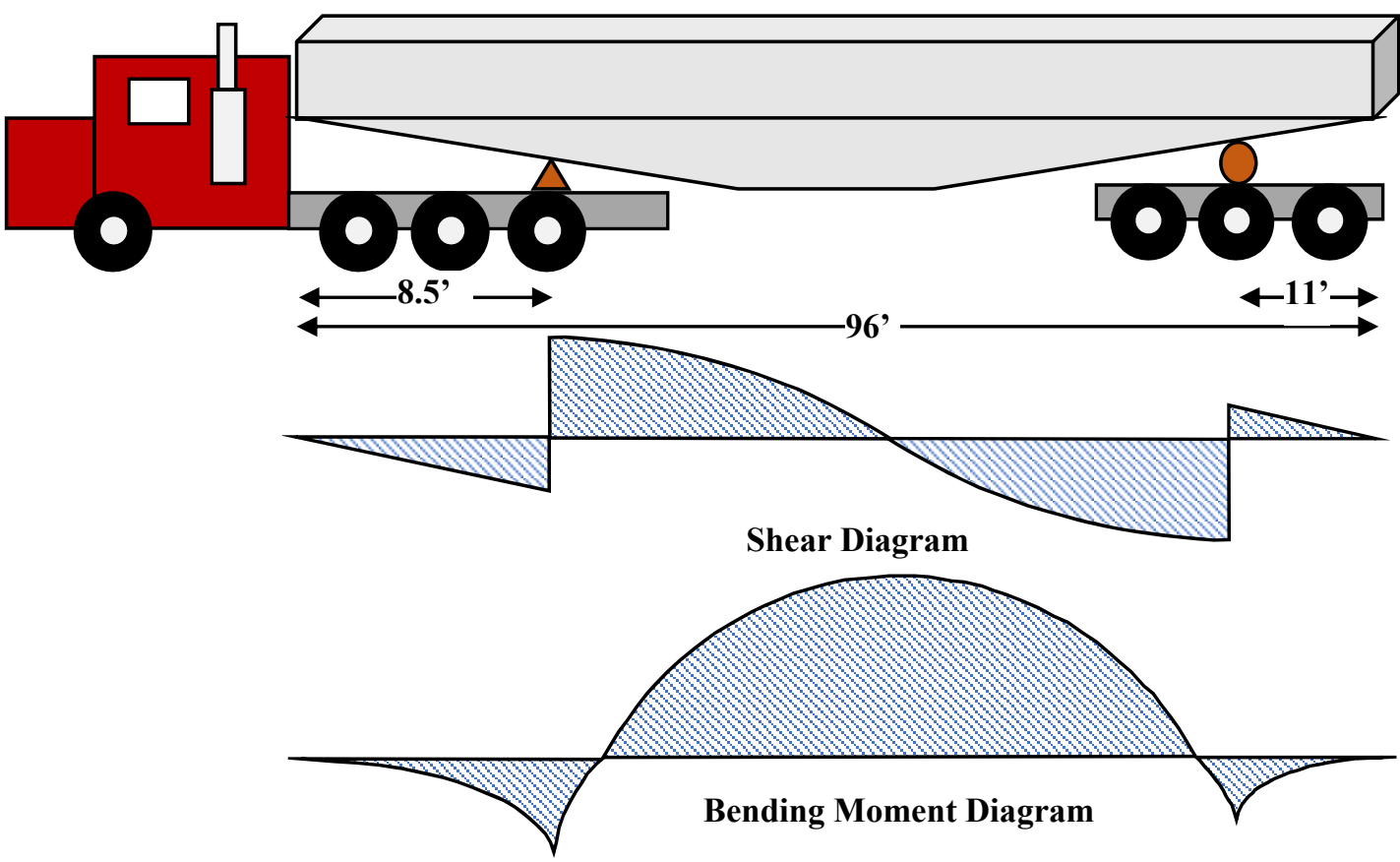

Figure 6.31. Hammerhead span NEBT shear and bending moment diagrams during transport

The locations that are in opposite loading between in-service loading and transport are between the transport supports as seen in comparison between Figures 6.31 and 6.32 .

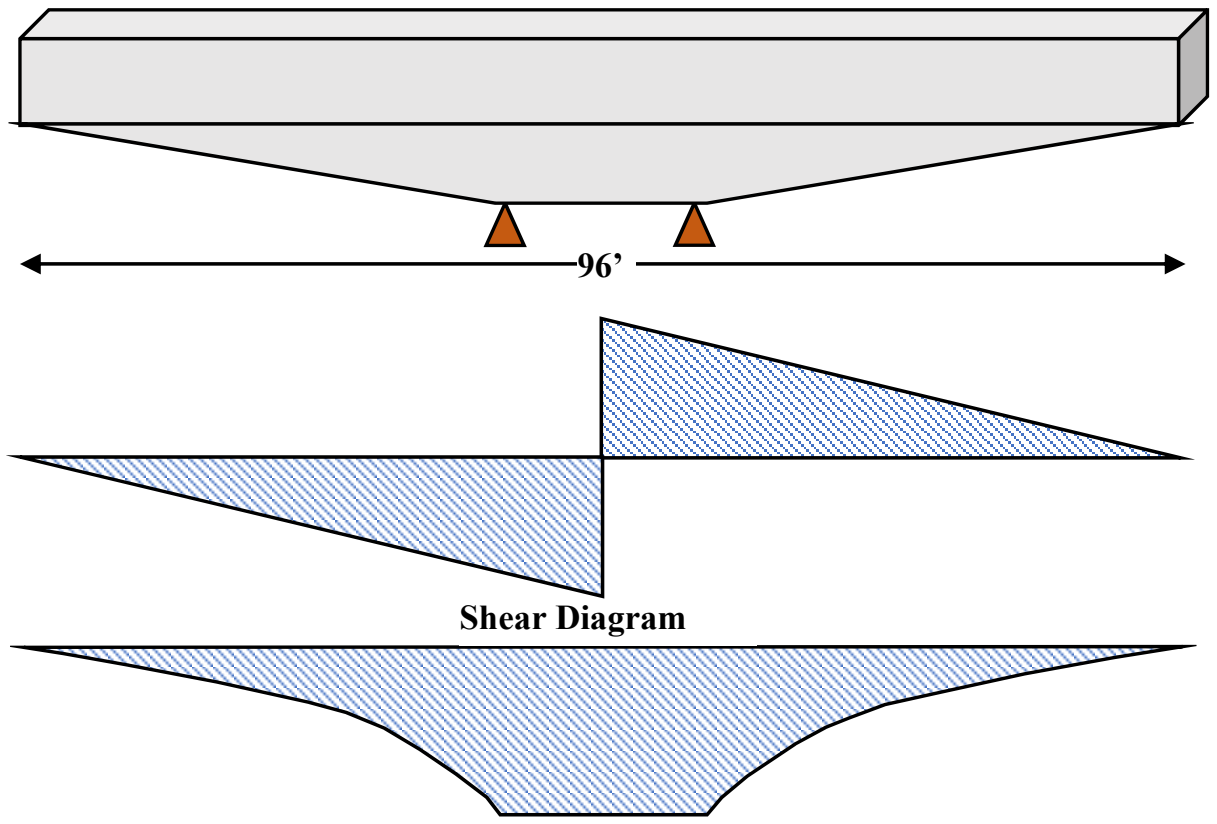

Bending Moment Diagram

Figure 6.32. Hammerhead span NEBT shear and bending moment diagrams during in-service loading 


\subsection{Conclusions from the Results of Transport Testing}

The results indicate that $\mathrm{AE}$ sensing is a potential practical $\mathrm{QA} / \mathrm{QC}$ process for PBES elements. The tests confirm the ability to collect AE data on prefabricated girders during transport. While there were no notable occurrences of damage or other QA/QC issues observed during transport, the recorded $\mathrm{AE}$ event distributions from the girder correspond to regions predicted by finite element modeling and simple shear/moment analysis to experience elevated stress levels. These results open the possibility for using the $\mathrm{AE}$ transport data to identify cracking and related damage that occurs during transport.

Field testing during transport of full-scale NEBT girders produced the following findings:

1. The AE test equipment required modest levels of custom modification for the transport tests.

2. The test equipment performed well during the tests and did not sustain any visible or notable damage.

3. Differences in AE event patterns were observed between end zone and middle zone regions along with between straight sections and hammerhead sections, Table 6.1.

4. Large quantities of AE events were recorded without correlation to observed cracks during transport.

5. B-value analyses may be an indicator or damage but additional testing is needed to determine the correlation between b-value and damage.

6. The amplitude lower limit threshold may need to be adjusted for future AE testing of hammerhead NEBT girders. 
7. Finite element modeling indicates areas of high stress corresponding to areas of dense AE event locations.

8. Additional data sets are required for statistical clustering and logistical regressions to define an $\mathrm{AE}$ event feature signature of cracks from other $\mathrm{AE}$ event source types.

Table 6.1. Quantification of AE Events Versus Girder Type and Sensor Array Location During NEBT Detensioning and Craned Movement

\begin{tabular}{lccc}
\hline Girder type & $\begin{array}{c}\text { Sensor array } \\
\text { Location }\end{array}$ & $\begin{array}{c}\text { \# of observed AE events } \\
\text { during transport }\end{array}$ & Damage Observed \\
\hline Straight NEBT & End span & 623 & None \\
Straight NEBT & Mid span & 2,628 & None \\
Haunched NEBT & Mid span & 91,723 & None \\
\hline
\end{tabular}




\section{CHAPTER 7 PROPOSED METHODOLOGY FOR QUALITY ASSURANCE / QUALITY CONTROL ACCEPT AND REJECT CRITERIA}

\subsection{Introduction}

The basis for most of the currently-available methods for damage assessment of reinforced concrete is the collection and analysis of experimental data sets derived from cyclic loading, often in an increasing pattern up to failure. In this vein, the Felicity ratio and Load-Calm ratios assess damage by examining AE activity in increasing loadingunloading cycles. Exceptions to the requirement for cyclic loading experiments are event counting and frequency analysis. An additional issue is the need for large data sets due to the inherent experimental scatter that characterizes most concrete fracture studies.

The fabrication and transport processes for Northeast Extreme Tee (NEXT) beams and Northeast Bulb Tee (NEBT) girders include; detensioning, craned form removal, and transport. These processes are single loading events (detensioning and craned form removal) or inconsistent/variable loading events (transport). The resulting data do not readily fit into a cyclic load testing framework. These differences in loading require modifying current damage assessment methods for use as a QA/QC procedure for the fabrication and transport events studied as part of this research. Due to the reliance on cyclic loading, damage assessments based on Felicity ratios and Load-Calm ratios are not directly applicable to these cases. Event-counting, parametric analysis, b-value analysis, and frequency analysis have better potential for adaptation for use in single loading events or inconsistent/variable loading. 


\subsection{Adapted Parametric Feature Analysis}

A feature-based analysis extracts and then records a set of features from each AE event waveform. The features include but are not limited to amplitude, duration, countsto-peak, and peak frequency. In a cyclic load-unloading regime, the correlation between RA values (risetime/peak amplitude) and the average frequency $(\mathrm{kHz})$ are established damage indicators. Single event threshold values have yet to be established. It is hypothesized that from the analysis of AE data during laboratory and field testing, thus far, that the use of statistical methods such as logistical analyses or multivariate analyses can establish feature threshold identifiers that would allow for the classification of a single AE event into the category of damage or no damage and distinguish the AE event source. To establish this feature analysis for "fingerprinting" of recorded AE events requires a larger AE data set than what was obtained as part of this study. A large AE data set of repeated testing is needed in conjunction with damage observations to establish not only the feature identifiers of different AE sources and levels of damage but also to establish reliability and typical variance for the prescribed $\mathrm{AE}$ event fingerprint.

\subsubsection{B-value and Frequency Analyses}

The b-value and frequency analysis were both developed as damage assessment tools but provide two very different interpretations of damage. The b-value analysis provides an assessment of the severity of damage while the frequency analysis provides a source of damage. Although the b-value analysis is a statistical regression of the AE event peak amplitudes and can be calculated for the loading cycle only or a complete loadingunloading cycle, it is hypothesized that if a large enough historical b-value data calculated from loading events was established, that lower and upper bounds for acceptable b-values 
could be established to assess the severity of damage. Once the damage severity is established from the b-value analysis the frequency analysis can be implemented to determine the source of damage. The frequency analysis flow chart as currently established will need revision to describe damage sources that are specific to damage modes possible during fabrication and transport processes for NEXT beams and NEBT girders. Similar to the parametric analysis, lower bounds for b-values and verification of the frequency analysis flow chart, along with the suitably; small variance requires a large AE data set. Repeated tests are needed in conjunction with damage observations to establishing upper and lower bounds.

\subsection{Potential AE Damage Assessment Methods}

Spatial representations of AE data collected during both laboratory and field testing yielded $\mathrm{AE}$ event locations that tended to cluster in areas of high stress or cracking and were diffuse in areas where no damage was observed. A correlation is hypothesized to be made between the number of concentrated $\mathrm{AE}$ events to an acceptable level of damage similar to the ASTM standard for AE testing of composite fuel tanks. AE technology has been proven to reliably detect material failure of composite fuel tanks and as such is an acceptable QA/QC technology for industry practice (ASTM E2191/E2191M-16). The use of $\mathrm{AE}$ sensing as a $\mathrm{QA} / \mathrm{QC}$ procedure in composite fuel tanks is a measure of cumulative recorded $\mathrm{AE}$ events over a prescribed amplitude threshold during a pressure test where the tank pressure incrementally increased with specific holding times between each pressure increase. If the number of recorded events exceeds a threshold, then the part is either slated for further examination (10,000 events) or rejected (50,000 events). Although only the detensioning process mirrors step loading, the same principle of increased AE activity as 
an indicator of stress and damage could be applied to many loading scenarios. Similar to the parametric and b-value/frequency analyses, a large $\mathrm{AE}$ data set of repeated testing is needed in conjunction with damage observations to establish an AE event density threshold to determine if the beam should be accepted or rejected but also to establish reliability and typical variance for the proposed $\mathrm{AE}$ event density method along with the variance of strength of the material being tested.

\subsection{Proposed Multi-Step Procedure}

A systematic future work effort would be multistep; 1 . Conduct realistic laboratory tests of beams with embedded defects so that they experience early and controlled damage and failure; 2. Identify the AE signatures of these failures; 3. Modify AE technique to improve sensitivity and selectivity of damage recognition; 4. Repeat these tests in a small pilot study to determine the experimental scatter of detection versus damage; 5. Analyze pilot test data to determine the statistical power of the technique and estimate the number of tests needed to establish validity of tests; 6 . Conduct sufficient number of repeat tests to identify sensitivity and selectivity in the form of Receiver Operating Characteristic (ROC) curves; 7. Identify field conditions prone to damage that is of concern and estimate the rate and severity of occurrence. This may be end zone cracking, delamination's, debonding, etc. 8. Combine information from the above steps to conduct a longitudinal study with sufficient number of repeats to obtain sufficient statistical power to justify setting an accept/reject threshold based on AE readings. Future work would be to conduct a large laboratory testing regiment over an extended period of time on a single type of commonly produced beam or girder to build a large AE database for all processes that are associated with cracking or high strain events. Once a large AE database is established with visual 
observations of damage, conditions, and element performance; the three theorized AE damage assessment techniques can be implemented and compared to determine the effectiveness for use as accept/reject criterion. Potential laboratory tests include; 1. Prestressed beam with diffuse cracking; 2. Beam with excess prestress that induces end cracking; 3. Beam with a crack prone mix.

\subsection{Conclusions}

Although there are many published damage assessment techniques for reinforced concrete, these techniques are based on cyclic loading and are not compatible with loading conditions observed during the fabrication and transport of the NEXT and NEBT beams. Loading of the full-scale prefabricated and pre-stressed, reinforced concrete NEXT beams and NEBT girders is either a single near-instantaneous event or a variable self-weight loading event. Of the five common reinforced concrete damage assessment techniques (felicity ratio; parametric analysis; load-calm ratio; b-value analysis; frequency analysis) only the parametric, b-value, and frequency analyses were determined to be adaptable to the field loading conditions experienced by the NEXT and NEBT beams during fabrication and transport.

Of existing AE data damage assessment techniques, there is one that is theorized to be applicable to the loading scenarios as observed in this research and two that are theorized to be used in conjunction with one another. Additionally, from AE data collected and analyzed as part of this study a third damage assessment technique is hypothesized. The three theorized damage assessment methods are;

1. The use of a parametric analysis using AE event waveform features to develop algorithms relating the unique features to observed damage types and severities; 
2. To build a database of $b$-values (using the published b-value damage assessment technique) for specific beams and processes. The database would indicate the upper and lower bounds for a typical b-value and therefore establish the upper and lower limits for accepting/rejecting the beam. Additionally, the frequency analysis would then be used to identify the AE source or damage type that lead to the rejection of the beam;

3. Based on AE data collected and analyses from laboratory and field testing, $\mathrm{AE}$ events tended to have densely clustered $\mathrm{AE}$ events near the location of observed cracking. It is hypothesized a damage assessment could be performed based on $\mathrm{AE}$ event clustering density. Once damage established the frequency analysis would then be used to identify the AE source or damage type.

In the case with all three of the theorized accept/reject damage assessment techniques, large $\mathrm{AE}$ data sets are needed for each beam type, reinforcement pattern, pretensioning strand pattern, and concrete mixture. Data sets need to include instances of varying observed damage from no damage to major damage in order to establish the lower and upper limits of acceptable $\mathrm{AE}$ emissions. It is not possible to establish accept/reject criterion based on the currently limited AE event database; however, the current database has presented a new and unique possibility for damage assessment as well as validated the efficacy of AE technology as a potential QA/QC procedure in locating the sources of $\mathrm{AE}$ events associated with cracking or high strain events. 


\section{CHAPTER 8 CONCLUSIONS AND FUTURE RECOMMENDATIONS}

This research had a variety of goals that were achieved through a mapped path of research objectives; designed to test the efficacy of AE sensing technologies as a Quality Assurance/Quality Control (QA/QC) procedure for the fabrication and transport of prefabricated and pre-stressed, reinforced concrete beams and girders. Conclusions drawn from the major research objectives are found in the text below along with recommendations for future work.

\subsection{Work Performed}

The following work was completed in support of the research presented in this thesis:

1. A commercial AE monitoring system was purchased and modified for field testing of prefabricated and prestressed, reinforced concrete during detensioning, craned lifting, and transport. Modifications included wiring to a portable power supply, connection to a wireless router for remote access, and the fabrication of metal U-shape mounting bracket for connecting and protecting the $\mathrm{AE}$ sensors to the concrete surface.

2. A laboratory pull-out test on a small reinforced concrete beam 5-inch $\mathrm{x} 5$-inch x 24-inch $(127 \mathrm{~mm} \times 127 \mathrm{~mm} \times 610 \mathrm{~mm})$ was performed to verify the functionality, capability, and deployment; along with to collect $\mathrm{AE}$ event location and feature data during a catastrophic failure event.

3. Two three-point bending tests were performed of small reinforced concrete beams 5 -inch x 5-inch x 24-inch (127 $\mathrm{mm} \times 127 \mathrm{~mm} \times 610 \mathrm{~mm})$, one with a 2$\mathrm{D}$ and the other with a 3-D acoustic emission sensor array layout to determine 
discrepancies and accuracies between the 2-D and 3-D sensor location algorithms.

4. Two field data collections events were performed on NEXT beams. The first data collection process used a 2-D sensor array on a NEXT beam held in the yard waiting to be transported. This test employed a pencil lead break test to determine the sensitivity of the $\mathrm{AE}$ monitoring equipment on full scale concrete beams and girders. The second data collection process used a 2-D sensor array and collected $\mathrm{AE}$ event location and feature data during both the detensioning and craned lifting processes.

5. Two field data collection events were performed during the fabrication of NEBT girders. The first data collection event used a 3-D rectangular grid sensor array and collected $\mathrm{AE}$ event location and feature data during both the detensioning and craned lifting processes. The second data event used a 2-D sensor array laid out in a triangular pattern and collected AE event location and feature data during both the detensioning and craned lifting processes.

6. Three field data collections events were performed during transport of both straight sections and haunch sections of NEBT girders from the fabrication location to the project site. The first data collection event used a triangular 2D sensor array pattern in the end zone of a straight NEBT girder. The second data collection event used a triangular 2-D sensor array pattern in the middle zone of a straight NEBT girder. The third data collection event used a triangular 2-D sensor array pattern in the middle zone of a hammerhead (haunch) NEBT girder. 
7. Finite element modeling (FEM) was performed in order to correlate recorded $\mathrm{AE}$ events to high stress areas.

\subsection{Overall Conclusions}

The following conclusions have been drawn from the research presented herein:

- A threshold filter of $60 \mathrm{~dB}$ is appropriate to filter out background noise during laboratory and field AE data collection.

- Accurate AE event source location depends on the source material wave mode velocities. Surface wave velocities can be measured through pencil lead break tests and longitudinal and transverse shear wave velocities can be estimated through published wave mode velocity correlations.

- The optimal AE sensor array is equilateral triangles and reduces the number of erroneous AE event location estimates.

- Of the fabrication processes, detensioning created the largest number of $\mathrm{AE}$ events and were diffuse in nature across the beam section indicating no apparent large stress concentrations.

- End zone cracking occurred near instantaneously during the detensioning process and end zone cracks did not appear to increase in size during craned lifting or transport.

- B-value analyses during detensioning show a strong potential for indicating damage.

- Transport testing of the end zone of straight section NEBT girders produced a diffuse pattern of AE events that mainly occurred in the upper half of the 
beam, which was estimated to be in light compression/tension during transport.

- Transport testing of the middle region of straight section NEBT girders produced a diffuse pattern of AE events that again mainly occurred in the upper half of the beam, which was estimated to be in light compression during transport due to the high pre-stressing forces.

- Transport testing of the middle region of hammerhead section NEBT girders produced a concentrated pattern of AE events that mainly occurred in the "belly" of the beam.

- B-value analyses on transport tests did not indicate any strong evidence of damage during transport.

- Recorded AE event locations were visually correlated with cracking during laboratory pull-out and three-point bending testing and field detensioning testing but were not correlated with cracking during field craned lifting and field transport testing as no cracks were observed during those data collection events.

- Finite element modeling indicates areas of high stress corresponding to dense AE event locations.

- Additional data sets are required for statistical clustering and logistical regressions to define an $\mathrm{AE}$ event feature signature of cracks from other $\mathrm{AE}$ event source types. 


\subsection{Intellectual Merit/Contributions to the Research}

This research made the following intellectual contributions to the state of the art:

1. As far as is known, this is the first acoustic emission research study that has collected acoustic emission data during the detensioning and initial craned lifting process of prefabricated and prestressed, reinforced concrete;

2. To the best of our knowledge, this is also the first acoustic emission research study that has collected acoustic emission data during the transport of prefabricated and prestressed, reinforced concrete from its fabrication location to installation site; and

3. Majority of literature in applying $\mathrm{AE}$ testing on concrete beams is on controlled cyclic loading tests; whereas, this research completed laboratory and field testing of single transient event loading.

\subsection{Broader Impacts of the Research}

Although $\mathrm{AE}$ sensing is currently a standard QA/QC procedure for the manufacturing of composite fuel tanks, this research was focused on the application of acoustic emission sensing as a potential $\mathrm{QA} / \mathrm{QC}$ procedure for prefabricated and prestressed, reinforced concrete beams and girders. This is to show the utility and broad spectrum of use of AE sensing. The testing and analyses performed for this research were specific to the NEXT beams and NEBT girders that have been uniquely developed by PCINE and local fabricators for the climate, loading conditions, and general use of northeast bridges. Although the beam designs are specific to the northeast region, the methodology and potential applications for acoustic emission sensing in prefabricated and pre-stressed, reinforced concrete beams and girders are universal. This technique can be broadened to other beam types, other materials, and other process applications. 


\subsection{Recommendations for Future Work}

Proposed future work includes additional laboratory testing, fabrication processes monitoring, and transport monitoring as well as the introduction for AE monitoring during beam placement and long-term service use monitoring. These continued and additional AE data collection scenarios are recommended to provide larger data sets for developing future $\mathrm{QA} / \mathrm{QC}$ standards and to complete $\mathrm{AE}$ monitoring during the life cycle through service use of a full-scale prefabricated and pre-stressed, reinforced concrete; 1) fabrication, 2) transport/construction, 3) service use.

Much of the focus of previous research on AE monitoring of reinforced concrete is centered around cyclic loading. Because of this, many of the statistical interpretation and damage assessment algorithms have been developed to require $\mathrm{AE}$ data from cycles of loading and unloading over time or with increasing load. Since most of the fabrication processes are single loading event cases, the cracking/damage assessment algorithms currently published are not applicable without modification. To develop these algorithms additional laboratory AE data collection would focus on:

1. Creating laboratory scale reinforced concrete beams that mimic full-scale beams in both geometry and reinforcement patterns;

2. Performing cycling loading-unloading tests to establish parametric AE event waveform criteria for cracking and damage based on established methods;

3. Collecting parametric AE event waveform data during loading to failure of the laboratory beams in manners simulating field fabrication processes (i.e. compression testing, three-point flexure testing, and pull-out testing); 
4. Establishing correlations between parametric AE event data collected in items 2 and 3 to develop cracking/damage algorithms for single loading event cases.

AE data collection during fabrication of full-scale prefabricated and pre-stressed, reinforced concrete NEXT beams and NEBT girders was focused on detensioning and craned removal from the formwork. AE data collected thus far during these fabrication processes is lacking duplicate data since each successive data collection event changed various aspects as the $\mathrm{AE}$ collection methodology. At a minimum three tests on each beam type using the identical test procedures and methodologies should be performed during each fabrication process.

Current AE data collection during transport is limited to NEBT girders and one test each of the end zone of a straight span beam, middle zone of a straight span beam, and middle zone of a hammerhead beam. This limited data set was due to timing constraints of beam transport for the Rockingham I-91 bridge construction project. Only three data collection events were possible and rather than repeat data collection, it was decided to collect different data sets in areas that would experience different stress distributions and magnitudes. It would be of interest to complete additional duplicate tests of the three already AE data collections during transport next construction season when the southbound bridge is constructed. Similarly, this would provide multiple AE data sets during transport for each of the beam regions tested; allowing for the development of algorithms to assess cracking/damage of beams during transport.

AE monitoring during beam placement was not performed during AE testing due to time constraints and needed coordination with the contractor. The Rockingham I-91 bridge construction project included the craned placement of NEBT girders from the 
staging area into to place on the abutments/bents. Since the placement of the NEBT's used a crane, there were safety concerns and coordination issues to disconnect and retrieve the $\mathrm{AE}$ monitoring equipment and sensors after placement. Ideally, the $\mathrm{AE}$ monitoring equipment would stay in-place from transport testing and would allow for consistency in $\mathrm{AE}$ data collection between transport $\mathrm{AE}$ data collection and placement $\mathrm{AE}$ data collection. AE monitoring of long-term service use was not performed in this study due to research scope, limited data storage space, and the need for a continuous power supply. $\mathrm{AE}$ monitoring of long-term service use would require additional $\mathrm{AE}$ channels to incorporate more $\mathrm{AE}$ sensors to provide a larger study area. Long-term service use monitoring would also require either cloud access for data storage or a larger hard drive for AE data storage. Additionally, either a cabled power source or solar power source would be required along with a battery back-up to provide continuous, uninterrupted $\mathrm{AE}$ data collection. Long-term monitoring at an exterior girder, interior girder, middle span, and end span would be recommended for data collection to cover all regions of the bridge. 


\section{CHAPTER 9 COMPREHENSIVE BIBILOGRAPHY}

Acoustic Emission Testing. (n.d.), NDT Resource Center, $<$ https://www.nde-ed.org $>$ (Jul. 14, 2018).

ARCHES Strategic Targeted Research Project. Assessment and Rehabilitation of Central European Highway Structures - Annex C: The acoustic Emission Method, pp. C$21-$ C-32. 2009.

ASTM E1316-18a, Standard Terminology for Nondestructive Examinations, ASTM International, West Conshohocken, PA, 2018, www.astm.org

ASTM E2191 / E2191M-16, Standard Practice for Examination of Gas-Filled FilamentWound Composite Pressure Vessels Using Acoustic Emission, ASTM International, West Conshohocken, PA, 2016, www.astm.org

ASTM E3100-17, Standard Guide for Acoustic Emission Examination of Concrete Structures, ASTM International, West Conshohocken, PA, 2017, www.astm.org

Aysal, U. (n.d.). "The Piezoelectric Effect.” Academia.edu - Share research, PZT Application Manual, $<$ http://www.academia.edu/9056282/PZT_Application_Manual_The_Piezoelectri c_Effect> (Sep. 18, 2018).

Baxter, M. G., Pullin, R., Holford, K. M., and Evans, S. L. (2007). "Delta T source location for acoustic emission." Mechanical Systems and Signal Processing, 21(3), 1512-1520.

Chen HL, He Y. (2001) "Analysis of Acoustic Surface Waveguide for AE Monitoring of Concrete Beams" J Eng Mech, V 127, N 1

ElBatanouny, M. K., Larosche, A., Mazzoleni, P., Ziehl, P. H., Matta, F., and Zappa, E., Identification of Cracking Mechanisms in Scaled FRP Reinforced Concrete Beams using Acoustic Emission, Experimental Mechanics, vol. 54, no. 1, pp. 69 82, Jan. 2014.

Grosse, C. U., and Ohtsu, M. (2008). Acoustic emission testing: [basics for research, applications in civil engineering. Springer, Berlin.

Hasenkamp, C. J., Badie, S. S., Tuan, C. Y., and Tadros, M. K., Sources of End Zone Cracking of Pretensioned Concrete Girders, 2008.

Head M, Efe S, Grose S, Drumgoole J, Lajubutu O, Wright R, Hansboro T. (2015) "Durability Assessment of Prefabricated Bridge Elements and Systems" Final Report, MD-13-SP309B4E, Maryland State Highway Administration, Office of Policy \& Research, 707 North Calvert Street, Baltimore MD 21202 
Hensman, J., Mills, R., Pierce, S. G., Worden, K., and Eaton, M. (2010). "Locating acoustic emission sources in complex structures using Gaussian processes." Mechanical Systems and Signal Processing, 24(1), 211-223.

Huang Q, Nissen GL. (1997) "Structural Health Monitoring of DC-XA LH2 Tank Using Acoustic Emission" Structural Health Monitoring Current Status and Perspectives, Technomic Publishing, Lancaster

Huston D., Structural Sensing Health Monitoring and Prognosis, CRC Taylor \& Francis, Boca Raton, FL, 2011.

Kaphle, M, Tan ACC, Thambiratnam, DP and Chan, THT, Effective discrimination of acoustic emission source signals for structural health monitoring, in Advances in Structural Engineering, 15(5): 707-716. 2012a

Kaphle, M, Tan ACC, Thambiratnam, DP and Chan, THT, Identification of acoustic emission wave modes for accurate source location in plate-like structures, in Structural Control and Health Monitoring 19(2): 187-198. 2012b

Krautkrämer, J. and Krautkrämer, H., Ultrasonic Testing by Determination of Material Properties, in Ultrasonic Testing of Materials, Springer, Berlin, Heidelberg, 1990, pp. 528-550.

Landis EN, Baillon L. (2002) "Experiments to Relate Acoustic Emission Energy to Fracture Energy of Concrete" J Eng Mech, V 128, 6

Lee, Y. H. and Oh, T., The Measurement of P-, S-, and R-Wave Velocities to Evaluate the Condition of Reinforced and Pre-stressed Concrete Slabs, Advances in Materials Science and Engineering, 2016.

MoDOT (2018). “751.22 P/S Concrete I Girders. 751.22 P/S Concrete I Girders,” Engineering Policy Guide.

NJIT Online RSS, "MSCE Articles.” (n.d.). , $<$ https://graduatedegrees.online.njit.edu/msce-articles/an-introduction-to-prestressed-concrete/> (Sep. 18, 2018).

Okumus P., Kristam R. P., and Arancibia M. D., "Sources of Crack Growth in Pretensioned Concrete-Bridge Girder Anchorage Zones after Detensioning," J. Bridge Eng., vol. 21, no. 10, p. 04016072 , Oct. 2016.

Okumus, P. and Olivia, M. G., Evaluation of crack control methods for end zone cracking in pre-stressed concrete bridge girders, PCI J., pp. 91-105, SPR 2013.

Physical Acoustics. Sensor Highway III Product Data Sheet, MISTRAS Group, Inc., Princeton, NJ. 2018 
Precast/Pre-stressed Concrete Institute Northeast. Guidelines for Accelerated Bridge Construction Using Precast/Pre-stressed Concrete Elements Including Guideline Details, 2nd Edition, pp. 1-48. 2014.

Ronanki V. S., Burkhalter D. I., Aaleti S., Song W., and Richardson J. A., "Experimental and analytical investigation of end zone cracking in BT-78 girders," Eng. Struct., vol. 151, pp. 503-517, Nov. 2017.

Sause, M. G. R. (n.d.). Investigation of Pencil-Lead Breaks as Acoustic Emission Sources, Journal of Acoustical Emission (29)., pp. 184-196, 2011.

Steel Auto Industries, "Supervision of Pre-stressing"|, $<\mathrm{http}$ //www.steelautoindustries.com/supervision-of-pre-stressing/> (Sep. 18, 2018).

Tensi, H., The Kaiser-Effect and its Scientific Background. Journal of Acoustic Emission, 22, pp.S1-S16. 2004.

Tuan C. Y., Yehia S. A., Jongpitaksseel N., and Tadros M. K., "End zone reinforcement for pretensioned concrete girders," PCI journal, vol. 49, no. 3, p. 68, 2004.

Vallen, H. (2009). “AE Testing Fundamentals, Equipment, Applications.” NDT.net, 7(9).

Vejvoda M. F., (2018) “Post-tensioning vs. Pre-tensioning," Post-tensioning Institute NPCA Education - PTI Seminar.

VTrans (2015) "VTrans Strategic Plan Mission, Vision, Goals and Objectives" Vermont Agency of Transportation, Montpelier, VT

Worley II, R. L., Dewoolkar, M. M., Xia T., Farrell R., Orfeo D., Burns D., Huston D. R., (2019) Acoustic emission sensing for crack monitoring in prefabricated and prestressed reinforced concrete bridge girders, ASCE Journal of Bridge Engineering, Special Edition. 


\section{CHAPTER 10 APPENDIX}

\subsection{MATLAB Codes}

The following MATLAB codes are examples codes and were used for raw data

processing from the AEwin ${ }^{\mathrm{TM}}$ Sensor Highway III and for plotting of data. Raw data files, images, and directory organization are not provided as part of this thesis.

\subsubsection{Example Code for Glyph Plots of Parametric Feature Data}

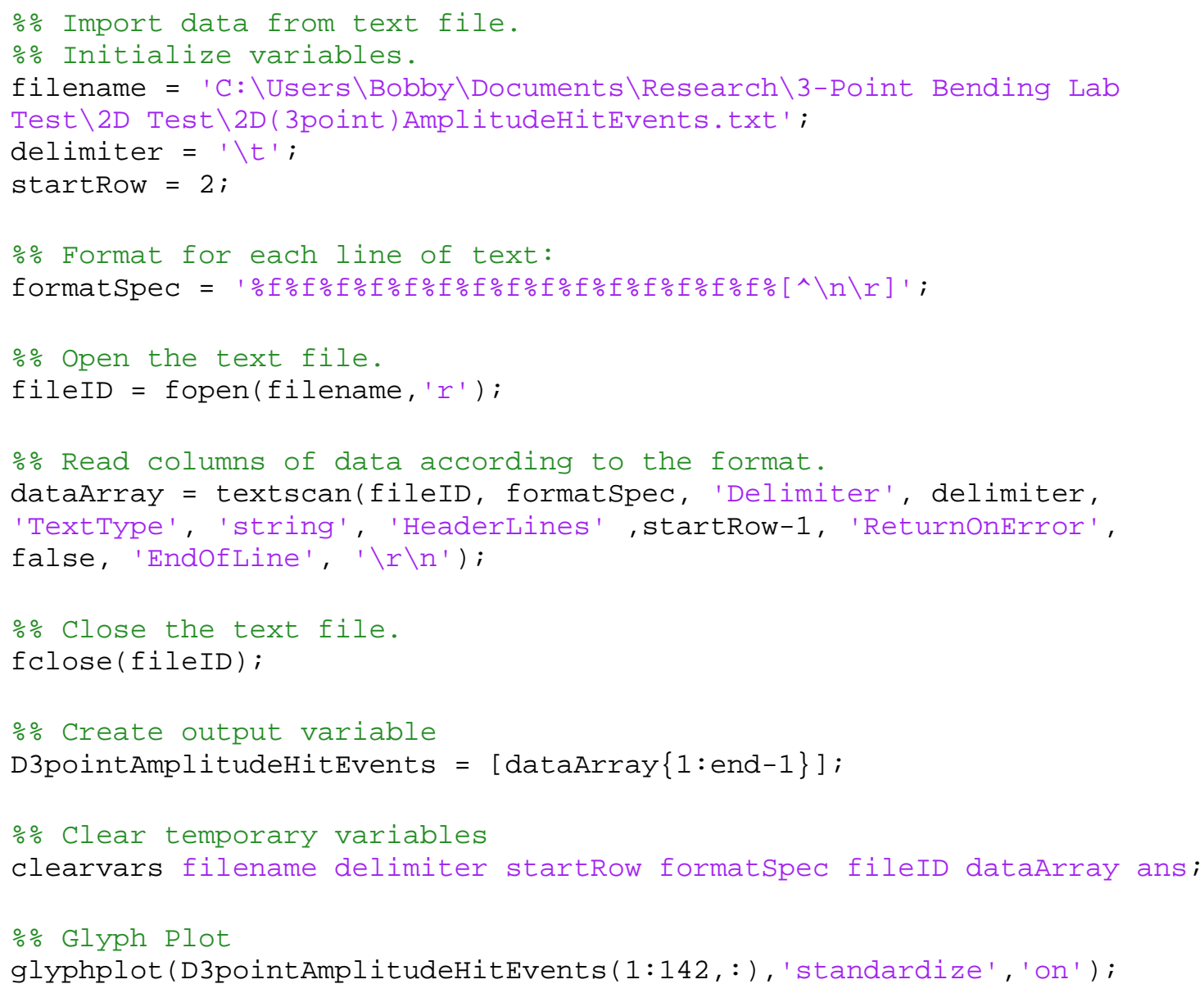

\subsubsection{Example Code for Extracting AE Event Location Data from Raw Data Files}

$\%$ Import data from text file.

$\%$ Initialize variables.

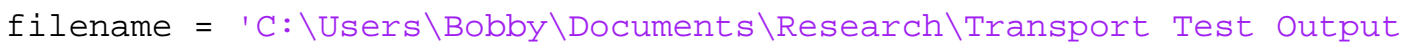
Files \TransportTest1EndofLongBeam. TXT' ; 


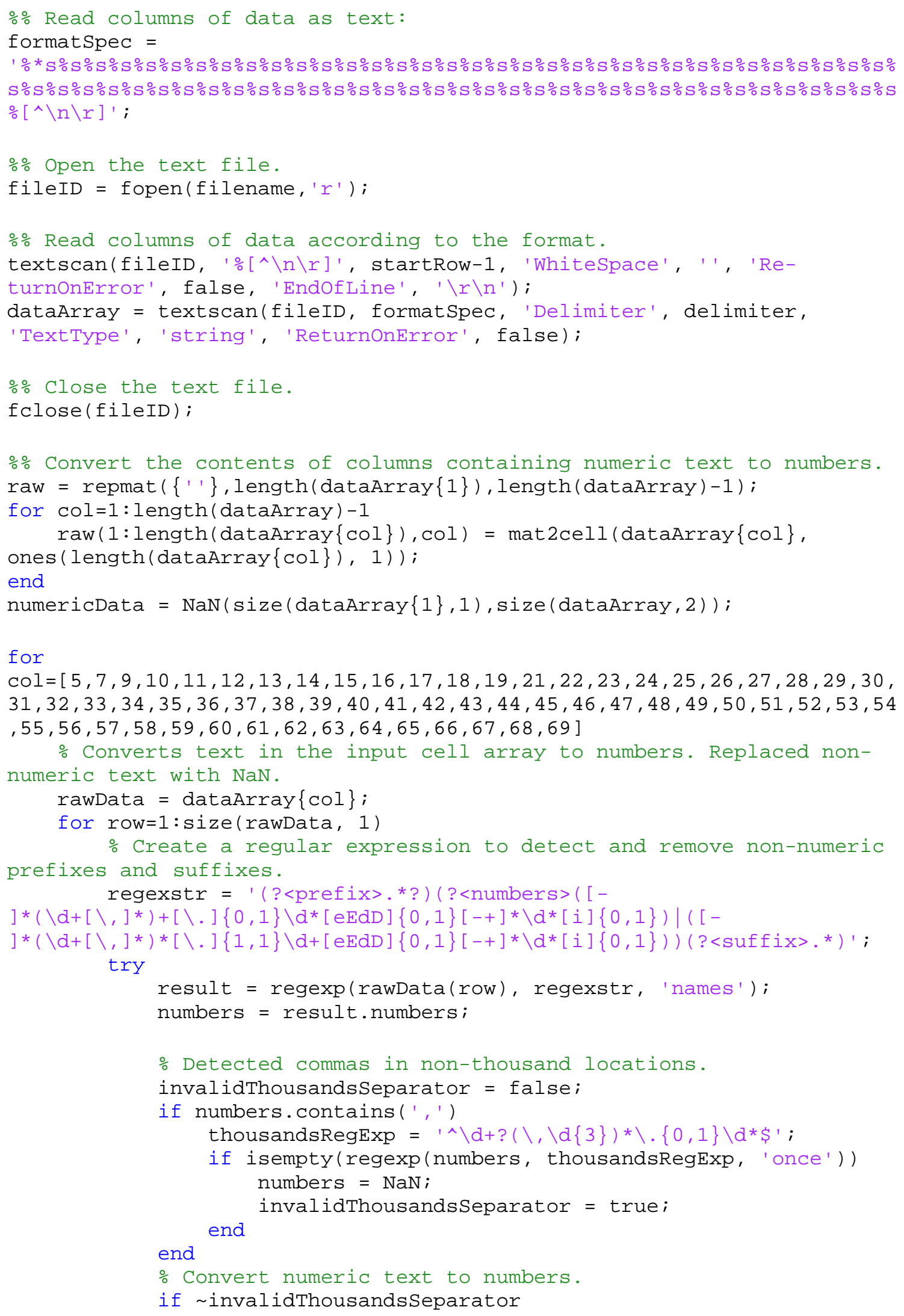




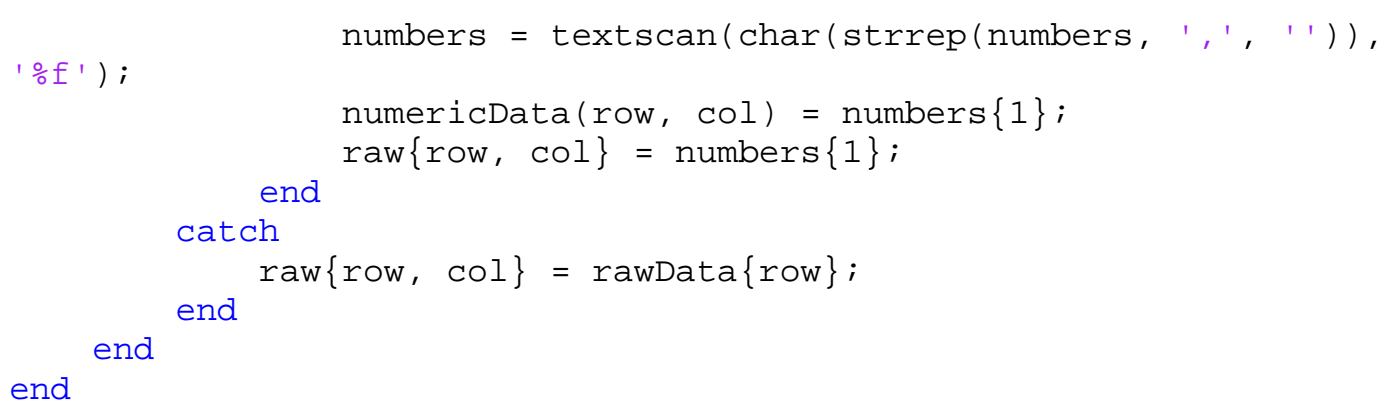


TransportTest1EndofLongBeam1.VarName13 = cell2mat ( rawNumericColumns(:, $6)$ ) ;

TransportTest1EndofLongBeam1.VarName14 = cell2mat ( awNumericColumns $(:$, 7));

TransportTest1EndofLongBeam1.VarName15 = cell2mat ( rawNumericColumns(:, 8));

TransportTest1EndofLongBeam1.VarName16 = cell2mat $($ rawNumericColumns $(:$, 9)) ;

TransportTest1EndofLongBeam1.VarName17 = cell2mat $($ rawNumericColumns $(:$, 10));

TransportTest1EndofLongBeam1.VarName18 $=\operatorname{cell2mat}($ rawNumericColumns $(:$, 11)) ;

TransportTest1EndofLongBeam1.VarName19 $=\operatorname{cell2mat}($ rawNumericColumns $(:$, 12));

TransportTest1EndofLongBeam1.VarName20 = cell2mat (rawNumericColumns $(:$, 13));

TransportTest1EndofLongBeam1. VarName21 = categorical(rawStringColumns $(:, 7))$;

TransportTest1EndofLongBeam1.VarName22 = cell2mat (rawNumericColumns $(:$, 14)) ;

TransportTest1EndofLongBeam1.VarName23 = cell2mat $($ rawNumericColumns $(:$, 15));

TransportTest1EndofLongBeam1.VarName24 = cell2mat $($ rawNumericColumns $(:$, $16)$ ) ;

TransportTest1EndofLongBeam1.VarName25 = cell2mat (rawNumericColumns $(:$, 17)) ;

TransportTest1EndofLongBeam1.VarName26 $=\operatorname{cell2mat}($ rawNumericColumns $(:$, 18)) ;

TransportTest1EndofLongBeam1.VarName27 $=\operatorname{cell2mat}($ rawNumericColumns $(:$, 19)) ;

TransportTest1EndofLongBeam1.VarName28 = cell2mat $($ rawNumericColumns $(:$, 20)) ;

TransportTest1EndofLongBeam1.VarName29 = cell2mat ( awNumericColumns $(:$, 21)) ;

TransportTest1EndofLongBeam1.VarName30 = cell2mat $($ rawNumericColumns $(:$, 22));

TransportTest1EndofLongBeam1.VarName31 = cell2mat $($ rawNumericColumns $(:$, 23)) ;

TransportTest1EndofLongBeam1.VarName32 = cell2mat $($ rawNumericColumns $(:$, 24)) ;

TransportTest1EndofLongBeam1.VarName33 = cell2mat $($ rawNumericColumns $(:$, 25)) ;

TransportTest1EndofLongBeam1.VarName34 = cell2mat $($ rawNumericColumns $(:$, $26)$ ) ;

TransportTest1EndofLongBeam1.VarName35 = cell2mat $($ rawNumericColumns $(:$, 27)) ;

TransportTest1EndofLongBeam1.VarName36 = cell2mat $($ rawNumericColumns $(:$, 28)) ;

TransportTest1EndofLongBeam1.VarName37 = cell2mat ( rawNumericColumns(:, 29));

TransportTest1EndofLongBeam1.VarName38 = cell2mat $($ rawNumericColumns $(:$, 30));

TransportTest1EndofLongBeam1.VarName39 = cell2mat $($ rawNumericColumns $(:$, 31)) ; 
TransportTest1EndofLongBeam1.VarName40 = cell2mat ( rawNumericColumns $(:$, 32));

TransportTest1EndofLongBeam1.VarName41 = cell2mat $($ rawNumericColumns $(:$, 33)) ;

TransportTest1EndofLongBeam1. VarName42 = cell2mat (rawNumericColumns(:, $34)$ );

TransportTest1EndofLongBeam1. VarName43 = cell2mat $($ rawNumericColumns $(:$, $35)$ ) ;

TransportTest1EndofLongBeam1.VarName44 = cell2mat $($ rawNumericColumns $(:$, $36)$ ) ;

TransportTest1EndofLongBeam1.VarName45 = cell2mat $($ rawNumericColumns $(:$, 37));

TransportTest1EndofLongBeam1.VarName46 $=\operatorname{cell2mat}($ rawNumericColumns $(:$, 38));

TransportTest1EndofLongBeam1.VarName $47=\operatorname{cell2mat}($ rawNumericColumns $(:$, 39));

TransportTest1EndofLongBeam1.VarName48 $=\operatorname{cell2mat}($ rawNumericColumns $(:$, 40));

TransportTest1EndofLongBeam1.VarName49 = cell2mat ( rawNumericColumns $(:$, 41));

TransportTest1EndofLongBeam1.VarName50 = cell2mat $($ rawNumericColumns $(:$, 42));

TransportTest1EndofLongBeam1.VarName51 = cell2mat $($ rawNumericColumns $(:$, $43)$ ) ;

TransportTest1EndofLongBeam1.VarName52 = cell2mat $($ rawNumericColumns $(:$, 44)) ;

TransportTest1EndofLongBeam1. VarName53 = cell2mat $($ rawNumericColumns $(:$, $45)$ ) ;

TransportTest1EndofLongBeam1.VarName54 = cell2mat $($ rawNumericColumns $(:$, 46));

TransportTest1EndofLongBeam1.VarName55 = cell2mat $($ rawNumericColumns $(:$, 47)) ;

TransportTest1EndofLongBeam1.VarName56 $=\operatorname{cell2mat}($ rawNumericColumns $(:$, 48));

TransportTest1EndofLongBeam1.VarName57 = cell2mat ( awNumericColumns $(:$, 49)) ;

TransportTest1EndofLongBeam1.VarName58 = cell2mat $($ rawNumericColumns $(:$, $50)$ ) ;

TransportTest1EndofLongBeam1.VarName59 = cell2mat (rawNumericColumns $(:$, 51));

TransportTest1EndofLongBeam1.VarName60 $=\operatorname{cell2mat}($ rawNumericColumns $(:$, 52));

TransportTest1EndofLongBeam1. VarName61 = cell2mat $($ rawNumericColumns $(:$, $53)$ ) ;

TransportTest1EndofLongBeam1.VarName62 = cell2mat (rawNumericColumns $(:$, $54)$ ) ;

TransportTest1EndofLongBeam1.VarName63 = cell2mat $($ rawNumericColumns $(:$, $55)$ ) ;

TransportTest1EndofLongBeam1.VarName64 $=\operatorname{cell2mat}($ rawNumericColumns $(:$, $56)$ ) ;

TransportTest1EndofLongBeam1.VarName65 = cell2mat $($ rawNumericColumns $(:$, $57)$ ) ;

TransportTest1EndofLongBeam1.VarName66 $=\operatorname{cell2mat}($ rawNumericColumns $(:$, 58)) ; 
TransportTest1EndofLongBeam1.VarName67 = cell2mat ( rawNumericColumns $(:$, 59)) ;

TransportTest1EndofLongBeam1.VarName68 $=\operatorname{cell2mat}($ rawNumericColumns $(:$, $60)$ );

TransportTest1EndofLongBeam1. VarName69 $=\operatorname{cell2mat}($ rawNumericColumns $(:$, 61));

TransportTest1EndofLongBeam1. VarName70 $=\operatorname{cell2mat}($ rawNumericColumns $(:$, $62))$;

\% Clear temporary variables

clearvars filename delimiter startRow formatSpec fileID dataArray ans raw col numericData rawData row regexstr result numbers invalidThousandsSeparator thousandsRegExp rawNumericColumns rawStringColumns $\mathrm{R}$ catIdx idx;

$\%$ Sort rows by location

LocationSort $=$ sortrows $($ TransportTest1EndofLongBeam1, 1)

\subsubsection{Example Code for Making a Video of AE Event Capture}

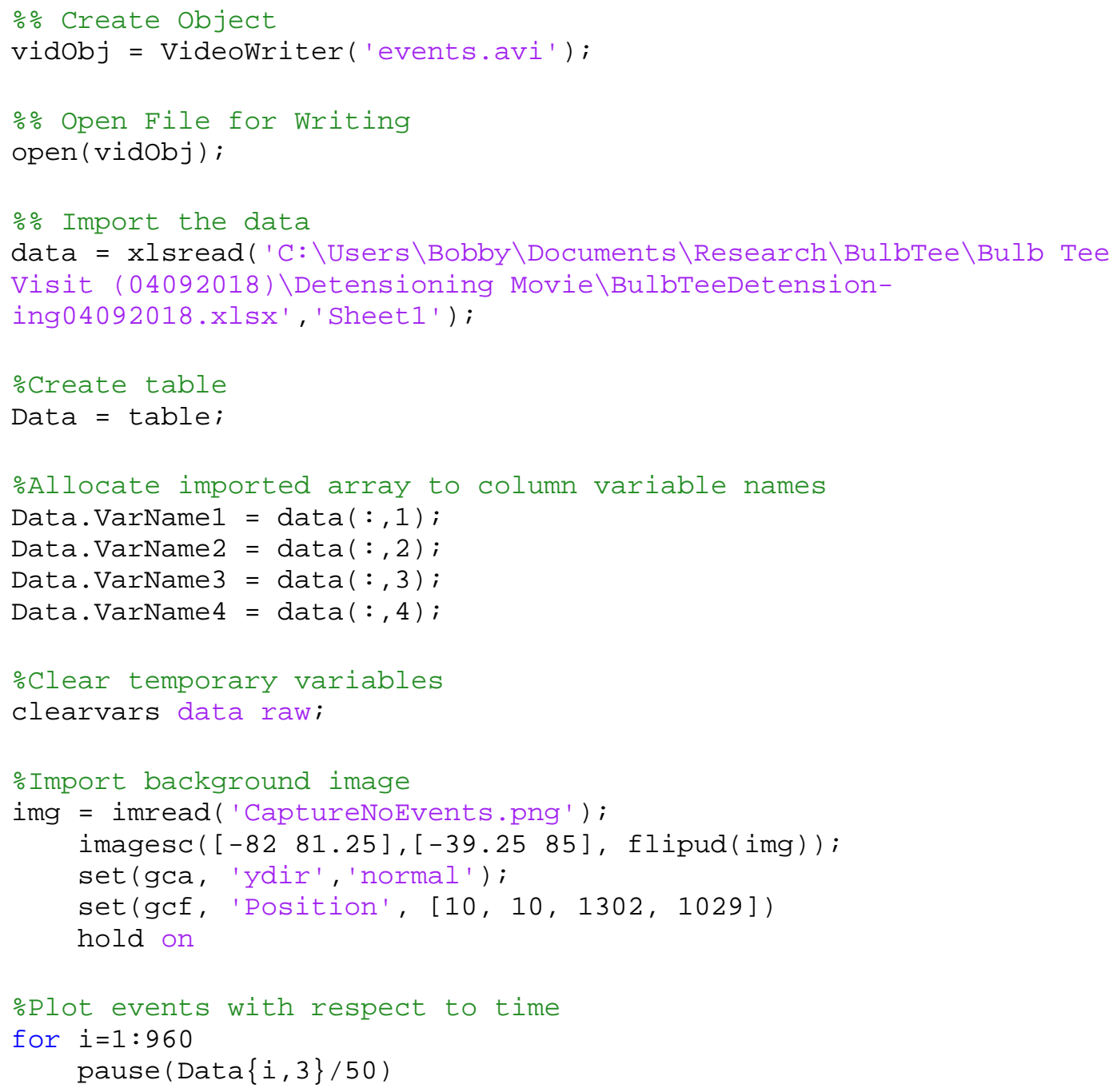




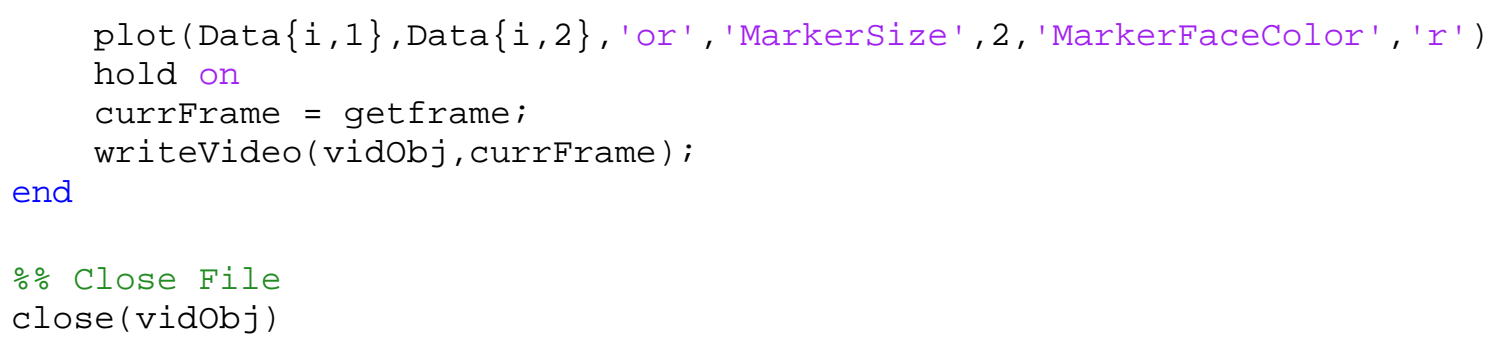

\subsubsection{Example Code for Plotting AE Event Locations and Source Amplitudes}

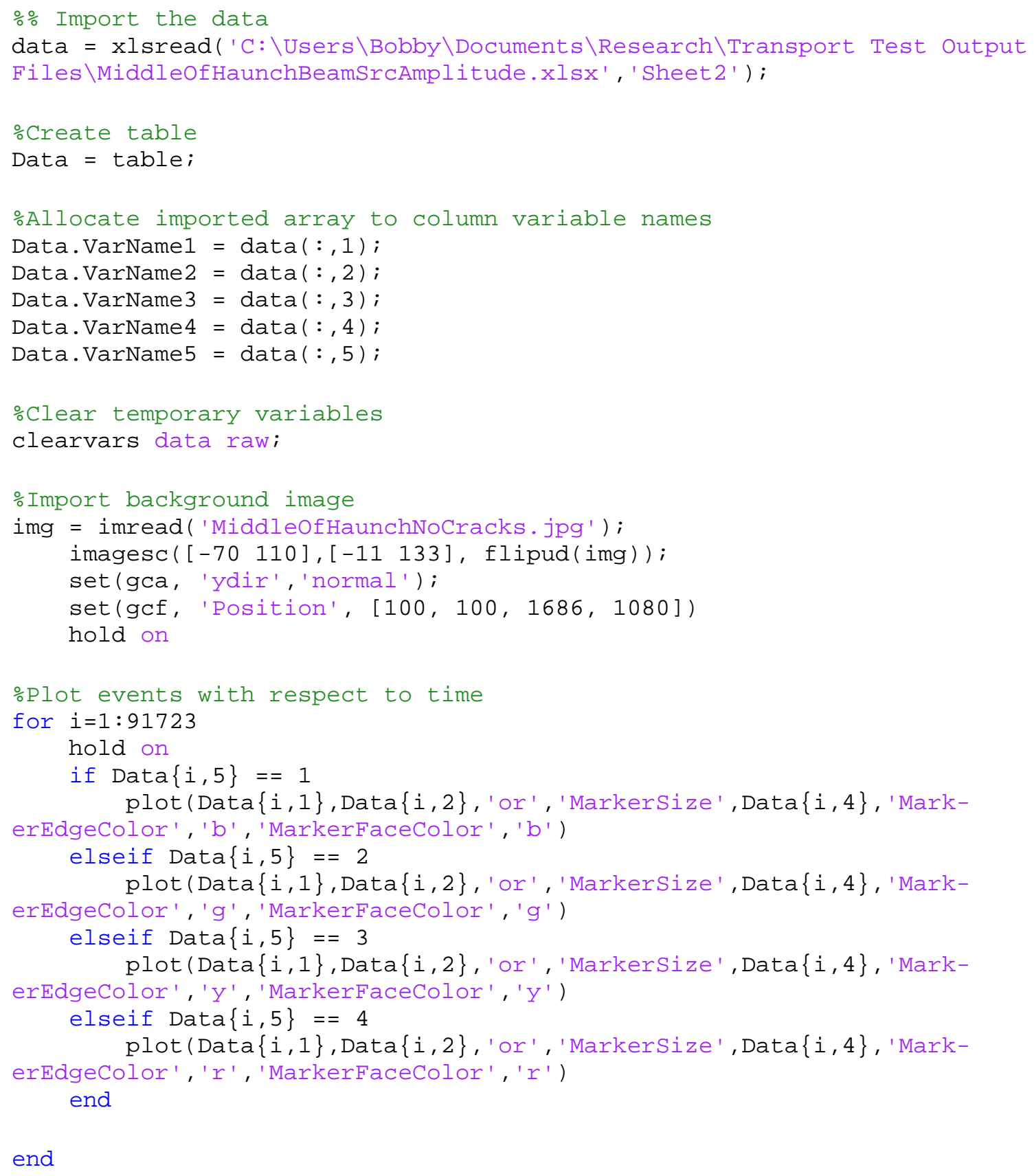

